https://helda.helsinki.fi

\title{
18th European Burns Association Congress
}

\section{Vuola, Jyrki}

Multidisciplinary Digital Publishing Institute

2019-09-03

Vuola, J. 18th European Burns Association Congress. EJBC 2020, 1, 3-190.

http://hdl.handle.net/10138/305258

Downloaded from Helda, University of Helsinki institutional repository.

This is an electronic reprint of the original article.

This reprint may differ from the original in pagination and typographic detail.

Please cite the original version. 


\section{8th European Burns Association Congress}

\section{Jyrki Vuola}

Helsinki Burn Centre, Department of Plastic Surgery, Helsinki University Hospital and University of Helsinki, PO. Box 800, FI-00029 HUS, Helsinki, Finland; Jyrki.vuola@helsinki.fi

Received: 31 August 2019; Accepted: 3 September 2019; Published: 3 September 2019

\section{Welcome Address}

Dear Friends,

The first time the European Burn Association Congress was held in the Nordic countries, was 16 years ago in 2003 in Bergen, Norway. Now we are back in the North for the second time and it is indeed a great honour.

The biennal EBA congress is the most important meeting place for burn professionals in Europe; but not only for us: participants from more than 40 countries all around the world are now gathering in the capital of Finland.

Over 360 abstracts guarantee an interesting programme along with comprehensive PAM sessions. We also have more than 25 distinguished guest speakers, an educational course and also a pre-congress EMSB (Emergency Management of Severe Burns) course that seems to be becoming more and more popular all across Europe.

The congress venue has an excellent location here in Helsinki with many attractions closeby, but hopefully not too tempting! However, the possibility of meeting people in a relaxed atmosphere is also very important. Networking is the key for success, but especially in burn care, where only a few people from different medical disciplines care for this rare patient group. This is particularly the case here in the sparsely populated northern part of Europe, whereby active communication between Scandinavian burn centres has become part of our routine.

On behalf of the Finnish team I warmly welcome you to Helsinki and wish you a most pleasant stay. If possible, don't miss the opportunity to visit a genuine Finnish sauna!

Jyrki Vuola,

EBA Congress President

\section{Abstracts Invited Faculty}

Invited Faculty (IF)

IF1.00 Implementing Major Burn Clinical Practice Guidelines Reduces Excess Fluid Administration at Non-Burn Centers

AA Papp, P Mankowski, K Genoway, S Gregory

Vancouver General hospital, VANCOUVER, Canada

Objectives: The severe inflammatory response and insatiable fluid losses associated with major burns requires aggressive fluid administration to maintain organ perfusion. In the province of British Columbia, Canada, clinical practice guidelines were implemented in 2011 to standardize resuscitation practices and optimize consistency in management prior to patient transport to a designated quaternary level burn center.

The objective of this study was to evaluate the impact in major burn fluid resuscitation since the implementation of the 2011 BC clinical practice guidelines.

Methods: A retrospective review of TBSA estimation, resuscitation records and clinical outcomes following implementation of the Adult Major Burns Clinical Practice Guidelines was 
conducted from 2011-2016. Study inclusion required patients older than 18 years of age with a TBSA burn injury greater than $15 \%$. Patients were categorized into groups based on treatment adherence to the 2011 guidelines and if these recommendations were initiated either peripherally or only after transfer to the quaternary burn centre. Results were compared to previously published BC burn outcome data prior to guideline implementation.

Results: Sixty-one patients met the study inclusion criteria. In the first $24 \mathrm{~h}$ after the burn injury, for patients that were initially managed peripherally and then transferred, they received an average $5.8 \mathrm{cc} / \mathrm{kg} / \%$ TBSA of fluid when there was no evidence of guideline adherence. If the guidelines were implemented only after transferred to our center, patients received an average of $5.0 \mathrm{cc} / \mathrm{kg} / \% \mathrm{TBSA}$ of fluid. For burn patients where guidelines were utilized both peripherally and after transfer, an average $4.0 \mathrm{cc} / \mathrm{kg} / \%$ TBSA was used for resuscitation. Complications such as respiratory failure $(17 \%$ vs. $11 \%$ ), abdominal compartment syndrome ( $4 \%$ vs. $0 \%$ ) and acute kidney injury ( $50 \%$ vs. $33 \%$ ) developed more frequently when the guidelines were only implemented after transfer to our center compared to when they were implemented at the center of initial presentation.

Conclusion: The use of new clinical practice guidelines to aid in fluid resuscitation following major burns has decreased IV fluid administration for patients initially assessed in peripheral low volume centers. Early guideline implementation additionally decreases burn associated systemic morbidities.

Picture $\quad 1: \quad$ https://www.eventure-online.com/parthenuploads/89/9EBA/add_1_560686_0a142232-dcf9-4016-a031-0e0b8c5816ba.png

\section{IF2.01 Burn Care: Where We Are and Where We Are Going}

\section{Palmieri}

Shriners Hospital for Children and UC Davis, SACRAMENTO, USA

Objectives: Burn injury is a major physiologic insult requiring a team-based approach integrating patient physiology with medical therapeutics. Major advances in burn patient survival in the past 50 years due to improvements in resuscitation and wound management have markedly decreased burn mortality, yet further advances are necessary, particularly for the elderly.

Methods: Urine output guided fluid resuscitation decreased mortality over the past 50 years, yet urine output is not the optimal resuscitation guide. Fluid creep remains a problem that impacts both circulatory and wound management, and incorporation of current technologies into fluid resuscitation is needed. Burn resuscitation has also benefitted from a second major advance in burn care: the advent of early excision with wound coverage.

Results: Early excision has decreased wound colonization and infection as well as fluid resuscitation requirements. The challenges of early resuscitation include accurate identification of wound depth, access to appropriate resources, and availability of donor sites. The role of early excision in the elderly is unclear. Newer technologies are being incorporated into wound care that will improve the diagnostic accuracy of wound depth and extent, thus enabling clinicians to adhere to a "do it right the first time" philosophy for wound management.

Conclusion: Other evolving technologies, such as skin substitutes and stem cells, will be the future for burn wound care. Burn care will focus on long term outcomes. Optimizing the burn outcomes of the future will require international partnerships to provide essential diverse perspectives on burn care.

\section{IF3.00 Goals in Wound Care}

\section{S.E. Wolf}

Shriners Hospital for Children-Galveston, GALVESTON, USA

Objectives:

* Describe the goals of wound care

* Discern the relevance of wound description

${ }^{*}$ Choosing timely, cost effective, and effort minimising treatments 
* Highlight the importance of effective treatments to decrease pain and suffering while maxmising long-term outcomes and outlooks.

Methods: Oral presentation

Results: Describe best goals and potential means to reach these.

Conclusion: Put our efforts into context

IF4.02 Why are trace elements important in burns

\section{Berger}

Lausanne University Hospital, LAUSANNE, Switzerland

Objectives: The early phase of major burns is characterised by an intense oxidative stress and lipid peroxidation, and significant cutaneous exudative losses. Very rapidly the patients present a metabolic turmoil, with increased nutritional needs causes by the initiation of tissue repair. The antioxidant defences require some specific micronutrients to be active: the trace element $\mathrm{Cu}$, Se and $\mathrm{Zn}$ are key components of this defence. The exudative losses contain large amounts of the 3 elements, which contribute as long as wounds are open, to an acute depletion of the endogenous stores: about $30 \%$ of the body's $\mathrm{Cu}$ content is lost in one week, $10 \%$ for Se and for $\mathrm{Zn}$. In addition to the increased needs associated with the oxidative stress, the nutritional requirements of the patients are increased: $\mathrm{Cu}$ and $\mathrm{Zn}$ are corner stones in the synthesis of collagen and elastin. Further local cutaneous antioxidation is important in the wound healing process. Finally, the immunity is depressed after major burns: here again the 3 trace elements $\mathrm{Cu}$, Se and $\mathrm{Zn}$ are cornerstones in the activity of leukocytes.

Randomised trials have been conducted, providing by the intravenous route from the day of injury the amounts of $\mathrm{Cu}$, Se and $\mathrm{Zn}$ lost in the exudates: repletion doses have been provided for 8 to 14 days depending on the burn size. The doses used in the trails have been 5-10 times superior to nutritional doses. The clinical results have been positive in terms of reduction of oxidative stress during the first week, improved graft take and wound healing, reduction of infectious complications and reduction of length of ICU stay. Therefore, the European ESPEN guidelines recommend the early intravenous delivery of trace elements with nutritional doses of vitamins to improve outcome

IF4.03 Misconduct in research - only in other families?

\section{B. Gerdin}

Uppsala University, UPPSALA, Sweden

Objectives: Close to one million new publications in the area of medicine are added to MEDLINE each year. All available information say that some of these contain false information. The number might only be speculated upon. An Editor to one of the most highly rated medical journals say: "may be up to half contain false information." An issue to discuss is whether there are reasons to suppose that this is different in the burn literature.

Reasons for reporting false information in papers are many. Some are due to "bad science", or due to honest mistakes of various kinds. Fraud during the process, or "misconduct in science", is probably a reason in only a minority of papers. Studies have given an estimate that about two percent of scientists have acted dishonestly in their science. This figure is much higher than the number of investigated cases of scientific fraud which suggest that most fraud are unrecorded and go under the radar. Outright fraud in science is distressing for many reasons. It give a false ground for further development of knowledge, may harm patients and damages public trust in science and in scientists. In understanding fraud in science, the significance of the very first step, or steps down a slippery slope to self-deception has been pointed out, and consequently the importance of giving young scientists a good ethic value base.

Picture $1:$ https://www.eventure-online.com/parthenuploads/89/9EBA/add_1_565403_abecf222-deb4-443b-8016-b45fddca1dda.png 


\section{Willebrand}

Uppsala university, UPPSALA, Sweden

Objectives: A burn is an unexpected life event that disrupts the illusion of safety in every-day life, changes life for both patient and family, and gives rise to existential concerns. Lately, there has been an increase in qualitative research focusing on experiences of burn injured persons. The aim here is to describe a synthesis of distinguishable existential themes.

Methods: A literature review was conducted. Selected studies had a phenomenological qualitative research approach and focused on experiences of burn injured persons.

Results: The burn is a boundary situation bringing the physical aspects of life to the fore, overshadowing other dimensions of life. The person struggles to find a new sense of normality by renegotiating embodiment, roles and identity, and to find a meaningful direction forward by creating a new coherent life story and employing new values. It is a prolonged shifting process as the wounds heal, pain and discomforts fluctuate, and psychological and social challenges arise. The new bodily situation changes how the person perceives the world and how the world perceives the person. Development is dependent upon the person's inner strength, but also on social connections, e.g. selfacceptance is dependent upon acceptant responses from others and vice versa. There is a striving to regain freedom, becoming independent, but not isolated. The injured person is deeply dependent on others for restoring a sense of safety, meaning and connectedness.

Conclusion: The physical aspects of life bring on an urgent need of developing a new sense of self in connection with the world. Balancing autonomy and dependence is a salient dilemma as neither can be discarded in a meaningful life. Implications for burn care are to help patients build bridges from the present to the future, by being-with in an empathetic way and helping to re-establish communication between the patient and significant others.

IF5.00 Translation and Implementation of Research in Burn Treatment

\section{E. Middelkoop}

Association of Dutch Burn Centers, BEVERWIJK, Netherlands

Objectives: Since several decades burn research has provided evidence for optimal treatment of burn injuries. Whereas 'How I do it' was indispensable to share experience and knowledge in the early days of the burn specialism, nowadays, Evidence Based Medicine and Practice are the lead.

Nevertheless, implementation of some of the results from clinical research shows a deficit.

We sought to clarify several aspects of why results remain 'on the shelf' based on the case of skin substitutes.

Skin substitutes such as Integra, Matriderm and others have been developed in the 1980's and have been on the market since the 90 's. Whereas initially evidence was limited, many clinical trial papers have been published since then. In total, close to 1500 papers have been published on Integra or Matriderm in scientific Journals. Even so, not every burn patient who needs a skin graft is treated with a skin substitute, while quality of healing would improve if treated so.

We thought financial restraints could be at the basis of this and therefore we studied the costs of skin substitutes in relation to total medical costs of burn treatment, and found that this was limited to roughly $10 \%$ of total. If this would lead to one less reconstructive procedure needed, the treatment with skin substitutes would be cost effective.

Several other aspects that could play a role in limiting implementation are: technical aspects (the need to change habitual procedures during surgery and aftercare), lack of exposure to the technology, limited follow up of patients. Especially the latter might be an essential factor, since 'own experience' still outweighs literature evidence.

\section{IF3.00 Goals in Wound Care}

\section{S.E. Wolf}

Shriners Hospital for Children-Galveston, GALVESTON, USA

* Describe the goals of wound care

* Discern the relevance of wound description 
* Choosing timely, cost effective, and effort minimising treatments

* Highlight the importance of effective treatments to decrease pain and suffering while maxmising long-term outcomes and outlooks.

IF6.01 Home Sweet Home ! The Importance of continued specialised rehabilitation within the community

\section{K. Whiting}

Birmingham Women's and Children's, BIRMINGHAM, Engeland

Objectives: Therapy input to the severely burnt individual starts on day one and continues throughout the burn survivors' journey. In the UK, burn therapists are required to adhere to national burn standards for assessment and treatment; the standards also enable therapists to plan and carry out truly individualised client centred treatment plans throughout their rehabilitation.

During the acute phase the burn survivor has intense daily input from multiple hospital-based disciplines; these inputs reduce as the individual moves into the out-patient phase and further reduce as they become re integrated into their community. It is this last phase where it is often the Occupational Therapist who remains the link between the client and the hospital.

The transition from In- patient to out- patient can often be stressful and frightening for families. The intense support that they receive during the acute phase dramatically reduces so that they can feel somewhat lost and alone. Practical and emotional problems can come to the fore once the family try to establish 'normal' life back in their home and community. It is therefore crucial that this area of rehabilitation is prioritised and that it is recognised as being as important as the rehabilitation provided in the acute phase.

Rehabilitation within the community setting can take many forms; factors that may influence the manner in which rehabilitation is delivered can be; differing ages, poor compliance, psychosocial issues, anxiety regarding return to education or work and restrictions imposed due to resistant bacterial infections. Examples of how an Occupational Therapist working within the NHS has used the burn standard framework to justify providing structured client centred treatment plans within the community setting will be discussed.

IF6.02 Developing E-Health in Burn Care in the Netherlands

\section{$M$ Veen}

Maasstad Ziekenhuis, ROTTERDAM, Netherlands

Objectives: The Dutch government wants to promote e-health. The Dutch Burn Association is creating a platform for this means.

Methods: Goals are set for digital access to medical files, performing measurements and possibilities to contact homecare 24/7 via screen in 2019. The project goes on in 2020 to make the best of developed apps and platforms.

Hospitals and other cure and care organisations make their patient content ready to share digitally and safely. Various platforms are being developed to make this information usable for patients.

Results: The Dutch Burn Associociation is developing a platform for patients with (severe) burns and necrotizing fasciitis, where they can access their medical files, information from their pharmacy and combine those with home care and their therapists. The platform will contain a chat function with selected professionals. Also information will be provided regarding common problems or questions in this patient category. On this subject patients and professionals are working together to cover as much ground as possible on different subjects. Furthermore known outcome in short term and long term, such as BORN, will be used in decision making tools. After summer 2019 the first preview of the digital platform is to be reviewed by the Dutch Burn Association.

Conclusion: Developing E-health is co-creation and a necessity in the near future. Long term use however raises some questions. 


\section{A Pittermann}

General Hospital of Vienna, VIENNA, Austria

Objectives: Internet and mobile-supported psychotherapeutic interventions (IMIs) represent the greatest advance in the development of psychotherapeutic/psychological therapy and a real paradigm shift in the way psychotherapy happens. The questions that arise from this are manifold and make it necessary to deal intensively with the opportunities and risks of this methodology.

Methods: In this talk, an overview of the development of internet- and mobile-supported psychotherapy will be given and in particular its applicability for burn patients will be reflected.

Results: While there are IMIs that represent a meaningful addition to the therapeutic work with burn patients, there are also IMIs whose application to burn patients does not seem to make much sense or which must be discouraged.

Conclusion: IMIs have become an integral part of the current development of psychotherapy. Their use in the psychotherapeutic treatment of burn patients poses a number of special challenges that must be considered. If these considerations are taken into account, there are good application possibilities for burn patients.

\section{IF7.00 Microsurgery in Burns}

\section{P. Barret}

University Hospital Vall d'Hebron, Universitat Autònoma de Barcelona, BARCELONA, Spain

Objectives: Reconstructive microsurgery has become an established discipline in plastic and reconstructive surgery. It is common practice in many areas of our specialty: head and neck, breast reconstruction, hand surgery, lower limb trauma reconstruction, etc. However, its utilization in acute burn surgery and burn reconstruction is sparse. Long series depict that only 1-2 \% of all patients admitted to burn centres receive microvascular flaps as part of their treatment. Microvascular surgery in burn patients, either acute or reconstructive, is a complex and difficult discipline, mostly because of the "burn problem": Hypermetabolism / Hypercoagulable state, Systemic inflammation, and many areas injured, grafted or simply destroyed and absent. The indications are commonly divided between PRIMARY (early stage, primary goal encourage wound healing) and SECONDARY reconstruction (improve functionality and aesthetics after healing of burn wounds). Timing is an important consideration, especially in primary reconstruction, and depends on: Mechanism of injury, anatomical location and extent of the injury. In order to avoid complications good preoperative planning is essential Take your time, be patience and stay away from area of injury. Use vein grafts if necessary and consider all possible options in flap design to achieve the desire outcome.

\section{IF7.02 Organisation of Burn Centres: The French experience}

\section{T. Leclerc}

Percy military teaching hospital, CLAMART, France

Objectives: The first French burn centre was created in 1951. After a peak around the turn of the millenium, the number of centres has decreased to a total of 19, including 15 in mainland France, for a total of 4 acute burn beds per million inhabitants.

Methods: In this talk, after a short epidemiological description of hospital admissions for acute burns in France, various features of the French organisation of acute burn care are reviewed, along with their potential strengths and weaknesses. In France, the organisation of the acute burn care network is regulated by national health authorities based on a limitative authorisation system designed in 2007 and on the verge of being updated. The funding of burn centres currently relies on a complex yet well designed diagnosis-based reimbursement policy by social security. Adult and pediatric patients are admitted to the same or separate facilities, depending on local choices and historical legacies.

Results: Those points are discussed, along with work in progress by the French health authorities together with the French burn society to prepare for a renewed organisation of the burn care network, including the project of multi-level acute care, and a better focus on post-burn rehabilitation. 
Conclusion: The governance of burn centres varies among hospitals and local situations, but several key points can be identified: a historically important yet decreasing role of intensivistsanaesthesiologists in burn centre direction, a trend towards concentration of structures either by regrouping burn centres or by merging burn ICUs and other ICUs, an always more insistant questioning by hospital managers of the integrated approach of burn care where dedicated professionals are gathered around those highly demanding patients, and more generally a growing importance of hospital efficiency-centred approaches that are sometimes only partly compatible with patient-centred approaches.

\section{IF7.03 Burn Guidelines and Burn Centers}

P. Vogt

Hannover Medical School, HANNOVER, Germany

Objectives: The EBA has made substantial efforts to further develop the standard of burn care and to harmonize the quality of treatment in Europe. Therefore guidelines haven been established which are continuously amended and revised. Also a verification process for burn centes has been initiated.

Methods: This program of the European Burn Association (EBA) is an evaluation program designed to verify a burn centre's resources that are required for the provision of optimal care to burn patients from the time of injury through rehabilitation. Elements of this voluntary program include an application, self-assessment questionnaire, an on-site evaluation by members of the EBA Certification Committee. A written report of the site visit team is reviewed by the EBA Certification Committee.

Results: The EBA finds that it is true a mark of distinction and could be an indicator to government, third-party payers, patients and their families, and verification organizations that the centre provides high quality patient care and meets the demanding standards for organizational structure, personnel qualifications, facilities resources and medical care services.

Conclusion: In conjunction with a comprehensive guidelines development program the EBA has developed a powerful and transparent program of quality assurance for burn therapy in Europe. Improvment of therapy, trends and also factors jeopardizing patients' outcomes may be identified more easily in the strive for better patient care.

IF11.00 Tracheostomy in Burned Patients: Why, For Whom, When And How?

\section{T. Leclerc}

Percy military teaching hospital, CLAMART, France

Objectives: Acute care of severely burned patients is a lengthy process that may involve prolonged mechanical ventilation. Whether this is done with simple endo-tracheal tube (ETT) or tracheostomy may vary depending on team preference.

Methods: This talk describes the rationale for tracheostomy, based on the limited available evidence. It summarizes the advocated or proven advantages and drawbacks of tracheostomy over ETT, regarding airway safety, procedure difficulty, complications, impact on ventilation weaning and infection risk. Based on these elements, it addresses the selection of patients for whom tracheostomy seems preferable with a focus on burn severity and inhalation injury, the optimal timing of the procedure, and most importantly the choice between surgical tracheostomy and percutaneous, optionnally fiberoptic guided, tracheostomy.

Results:

Conclusion: On the whole, in order to reduce the risk of accidental loss of airway, to improve patient comfort and safety, and maybe to facilitate weaning and reduce the risk of ventilation acquired pneumonia, it seems sound to perform an early (before H48) fiberoptic guided percutaneous tracheostomy in severely burned patients for whom mechanical ventilation is expected to last 14 days or more. 


\section{K W Dunn}

Manchester Foundation Trust, MANCHESTER, United Kingdom

Objectives: The long-term collection of carefully structured data from all burn services within each health economy (usually national) allows several important functions to be fulfilled when sufficient data has been accumulated. These include:

An ability to understand the demand for burn services alongside an assessment of the current use of the existing capacity to identify either economies of scale or occasional rebalancing of services.

A clear understanding of how burn services should be optimally organised to meet the demands of their catchment population.

The ability to serially assess factors which impact on mortality and length of stay amongst the burn care population.

An opportunity to develop quality assurance measures and assessment of services against standards developed by consensus to indicate whether services are behaving optimally and in some instances identify outlier services that may require additional support or guidance.

In the longer term it also allows the monitoring of the epidemiology of burn injury and the effectiveness or otherwise of prevention strategies, recognising that observations from a single service or a small group of services remain unconvincing.

The value of centralised information gathering about burn service activity in sufficient detail to inform these issues, amongst others such as performing power calculations for clinical research projects are powerful arguments for the creation and long term maintenance of such systems in all health economies globally.

\section{IF19.00 Phage Therapy Initiative in Finland}

\section{Skurnik}

University of Helsinki, Helsinki, Finland

As the world is facing the threat of increasing antibiotic resistance, phage therapy, the 100 year old remedy of bacterial infections practiced in former Soviet Union and Russia, has been seen as a possible solution. In phage therapy, to control bacterial infections, lytic phages after reproducing itself within the target bacterium the phage kills its host and infect close-by neighboring host bacteria until all target bacteria have been eliminated. Unlike antibiotics, this does not disrupt the normal microbiota. In addition to cure bacterial infections phages can be used prophylactically to control bacterial pathogens in food production. I will also briefly present our progress in the phage therapy intitative that we started in 2013 in Finland. We have collected and characterized phages against clinical drug resistant bacterial pathogens. We have set up a phage purification pipeline to be used for phages specific for single patient isolates.

\section{IF19.00 Phage Therapy: Perspective for the Treatment of Infected Burns}

\section{T. Leclerc}

Percy military teaching hospital, CLAMART, France

Objectives: Phage therapy is the highly specific treatment of bacterial infections with bacteriophages.

Methods: This talk first summarises the results and lessons learned from our PHAGOBURN study (NCT02116010 ; Jault et al. Lancet Inf Dis 2019 ; 19(1): 35-45), the first ever multi-center, double blinded, randomised controlled trial evaluating efficacy and tolerability of phage therapy in infected burned patients. In this trial, adult patients with confirmed P.aeruginosa burn wound infection were randomly assigned to a 7-day topical daily treatment with either a cocktail of anti-P.aeruginosa bacteriophages (PP1131) or silver sulfadiazine (SSD) as control, with 14 days of follow-up.

Results: Between July 2015 and January 2017, 27 patients were included. The trial was then stopped for insufficient efficacy of PP1131. In the 25 patients analysable for efficacy (modified intention-to-treat population, P1131 n=12, SSD n=13), a sustained reduction of bacterial burden was achieved in a median of $144 \mathrm{~h}$ (95\% CI 48-not reached) in the PP1131 group versus a median of $47 \mathrm{~h}$ (23-122) in the SSD group (hazard ratio 0,29, 95\% CI 0,10-0,79; p=0,018). PP1131 hence decreased 
bacterial burden in burn wounds at a slower pace than SSD, but PP1131 titre decreased after manufacturing and participants actually received a more than $10^{3}$ lower concentration of phages than expected.

Conclusion: Beyond those results, based on the most fruitful lessons learned from that study and few others, perspective and proposed guidance for further application of phage therapy in burn wound and other infections are developed, with a special focus on phage selection and bioproduction, and on optimizing strategies of phage therapy by the use of tailored treatments based on phagograms.

\section{Abstracts Oral Presentations (O)}

O1.01 Time to First Excisional Debridement: Findings from the Burn Registry of Australia and New Zealand

HJ Cleland ${ }^{1}$, Y Singer ${ }^{1}$, B Gabbe ${ }^{2}$, P Cameron ${ }^{2}$, F Wood ${ }^{3}$, T Perrett ${ }^{4}$, L Tracy ${ }^{2}$

${ }^{1}$ Alfred Hospital, MELBOURNE, Australia

${ }^{2}$ Monash University, MELBOURNE, Australia

${ }^{3}$ University of Western Australia, PERTH, Australia

${ }^{4}$ Counties Manukau Health, AUCKLAND, New Zealand

Objectives: Early excision may modulate the acute systemic inflammatory response of large burns, and is associated with improvements in survival, complication rates and hospital length of stay. The aim of this study was to determine whether variation exists in the time to first excisional debridement across ANZ burn centres and the effects of variation on patient outcomes.

Methods: Data were extracted for acute admissions between July 2016 and June 2017 from the Burn Registry of Australia and New Zealand (BRANZ).

Of the 4,992 cases, 3,840 patients $(77.1 \%)$ had a surgical burn wound management procedure in theatre, and more than half of these patients underwent excisional debridement.

Results: For all patients, the median time to first excision (from time of injury) was 6.41 days (interquartile range (IQR) 2.9 - 12.2 days). After risk adjusting for patient age, gender, primary cause of burn injury, TBSA, inhalation injury, and burn depth, two sites were more than two standard deviations from the risk adjusted mean time to first excision (one above, one below). For patients with a burn equal to or greater than $20 \%$ TBSA, the median time to first excision was 1.69 days (IQR $0.91-4.03$ days).

Conclusion: Variation exists in surgical practices between ANZ burns units. The reasons for this are unclear, and further examination is warranted to understand effects on outcomes and potential for improved practice.

O1.02 Burns during Pregnancy: A Systematic Review and a Proposal for Guideline

A Pijpe ${ }^{1}$, ML Dijkerman $^{1}$, ML Walters-Breederveld ${ }^{1}$, RS Breederveld ${ }^{2}$

${ }^{1}$ Red Cross Hospital, BEVERWIJK, Nederland

2Leiden University Medical Center, LEIDEN, Nederland

Objectives: Although there is numerous evidence regarding the implications and management of burns in general, guidelines on management of burn injuries during pregnancy are lacking. Due to the low incidence, clinical experience is limited. The pregnancy-related hormonal and physiologic changes and the presence of a fetus makes burn management challenging. There are many issues related to both the mother and the fetus to consider. The aim of our study was to perform a systematic review on management and outcomes of burns during pregnancy. Subsequently, a guideline to aid treatment decision making is proposed.

Methods: A literature search was performed in Pubmed, Embase, Cochrane and Web of Science until June $14^{\text {th }}$, 2018. Search terms: burns, pregnancy, pregnant women and fetus. Inclusion criteria: pregnant women main study population, $\geq 1$ clinical outcome, English and Dutch. Screening, data extraction, and risk of bias assessment were performed by two independent investigators. Conflicts were solved by a third investigator. Data extracted included study and population characteristics, 
treatment and supportive care, and outcomes of mother and fetus. This systematic review protocol was registered at PROSPERO. The PRISMA statement guidelines were followed.

Results: Preliminary results show that management of burns in pregnant women differs from non-pregnant women. The factors that must be considered include fluid resuscitation, length of stay, antibiotics, local and surgical treatment. The total body surface area burned is positively associated with maternal death, and therefore fetal death. Early induced delivery may be vital to save both mother and child. Specific attention should be placed on anaesthesia and pain management, taking into account the fetal effects. Local treatment should be adjusted due to possible teratogenic effects after the second trimester.

Conclusion: The management of burns during pregnancy requires an intricate balance between effectively treat the mother and minimizing the harm for the fetus.

O1.03 NexoBrid Enzymatic Debridement - Comparison between Pediatric and Adult Clinical Trial Results

$\underline{\text { Y Shoham }}^{1}$, F Sander ${ }^{2}$, I Konigs ${ }^{3}$, Y Krieger ${ }^{1}$, E Silberstein 1 , T Ayzenberg ${ }^{1}$, E Maor ${ }^{1}$, L Rosenberg ${ }^{4}$ ${ }^{1}$ Soroka University Medical Center, BEER SHEBA, Israel

${ }^{2}$ Unfallkrankenhaus Berlin, BERLIN, Germany

${ }^{3}$ Altonaer Kinderkrankenhaus \& University Medical Center, Department of Pediatric, HAMBURG, Germany

${ }^{4}$ Meir Medical Center, KFAR SABA, Israel

Objectives: The aim of this study is to compare the results of children vs adults in NexoBrid (NXB) trials.

Methods: Mixed populations of children and adults were enrolled in 3 of 7 former NXB trials:

Phase II - 77 children and 77 adults suffering from deep burns were treated with NXB in a prospective, single arm, single-center trial.

Phase III - 33 children and 148 adults suffering from deep burns were treated with NXB or SOC as part of a multi-center RCT. Seventeen children and 83 adults were treated with NXB. Sixteen children and 65 adults were treated according to the investigators' SOC.

Phase IIIb - 17 children and 72 adults treated in the RCT were available for a 2-4 year post injury long term follow-up.

Results: Phase II: Pediatric results were slightly better vs adults; incidence of complete eschar removal ( $92 \%$ vs $91 \%$ ), need for autografting ( $34 \%$ vs $42 \%$ ), and days to complete wound closure (21.4 vs 22.2).

Phase III: Most of the results of NXB treated patients were significantly better than SOC patients. This NXB/SOC effect was even more pronounced in children vs adults in most parameters; incidence of complete eschar removal $(100 \% / 94 \%$ vs $88 \% / 89 \%)$, a 9.1 vs 3.6 times reduction in the area that underwent surgical eschar removal, a 4.2 vs 2.2 times reduction in DPT areas that underwent autografting, a 2.5 vs 1.8 times reduction in blood loss, and less days to complete wound closure.

Phase IIIb: MVSS scores were also in line with the more pronounced NXB/SOC effect in children vs adults (3.39/4.37 vs 3.07/3.07). There was a significant reduction in the presence of donor site scars in the overall population ( $40 \%$ NXB vs $68 \%$ SOC) and lower scar modulation and reconstruction rates.

Conclusion: The results of children treated by NXB appear to be even better than in adults.

Picture

1 : https://www.eventure-online.com/parthenuploads/89/9EBA/add_3_538123_c93f5ed8-5a79-4292-9565-d006a75ce400.png

Picture 2: https://www.eventure-online.com/parthen-uploads/89/9EBA/add_538123_c93f5ed85a79-4292-9565-d006a75ce400.png

Picture 3: https://www.eventure-online.com/parthen-uploads/89/9EBA/add_1_538123_c93f5ed85a79-4292-9565-d006a75ce400.png

Picture 4: https://www.eventure-online.com/parthen-uploads/89/9EBA/add_2_538123_c93f5ed85a79-4292-9565-d006a75ce400.png

O1.04 Blood Transfusions Requirements in Burn Surgery 
$\underline{\text { Tichil }}^{1}$, H Cleland ${ }^{2}$

${ }^{1}$ County Emergency Hospital Cluj-Napoca, CLUJ-NAPOCA, Romania

${ }^{2}$ Alfred Hospital, MELBOURNE, Australia

Objectives: Analysis of blood transfusion requirements in the acute setting of burn injuries and an overview of transfusion rates, triggers, targets, risk factors and outcomes.

Methods: Over a 6-year period all acute admissions to the Victorian Adult Burns Service have been reviewed and 948 patients who have undergone excisional surgery have been selected as the focus for our analysis. For all eligible patients demographic data has been collected along with associated comorbidities, burn injury details, theatre procedures, and recorded transfusions.

Results: Our data showed that out of all the patients to undergo surgery $18.45 \%$ required a blood transfusion in and or out of theatre. Average haemoglobin throughout admission for these patients has been $97.42 \mathrm{mg} / \mathrm{dL}$ (IQR: 92.07, 105.16). The majority of transfusions, 52\%, occurred in a perioperative timeframe. Multivariate analysis has shown that patients who required blood transfusions had increased length of hospital stay (regression coefficient 0.511 , standard error 0.085, $\mathrm{p}<0.0001)$. Multiple logistic regression analysis has shown that age, gender, TBSA, number of surgeries, the presence of inhalation injury, associated coagulopathy and cardiac comorbidity as well as length of ICU stay are independent risk factors for blood transfusions.

Conclusion: Anemia is a common feature of acute burn injuries and blood transfusions are essential to surgical management. However, blood is a finite resource and its use is associated with numerous complications, therefor on-going interest and research promotes the development of guidelines to safely reduce transfusion trigger levels. Given that most often transfusions are related to surgical procedures the employment of blood conserving strategies may reduce transfusion rates in stable patients. As such, blood transfusions may be considered a potential quality indicator in burn care.

O1.05 Full-Thickness versus Glabrous Split Thickness Skin Grafts in Paediatric Contact Burns of the Palm: Comparative Analysis of Functional and Cosmetic Outcomes

JElrod, D Möllmeier, C Mohr, C. Schiestl

Pediatric Burn Center, University Children`s Hospital Zurich, ZURICH, Switzerland

Objectives: Palmar burns, although not life-threatening, can have a significant impact on the function and aesthetics of the hand. The aim of this study was to compare the use of full thickness skin grafts (FTSG) from the retroauricular region with split thickness skin grafts (STSG, 0.5mm) from the unloaded plantar foot in terms of function and aesthetics in children with deep dermal burns of the palm.

Methods: 32 transplants of the palm in paediatric burn patients, including 19 retroauricular FTSG (group 1) and 13 STSG from the unloaded plantar foot (group 2) were investigated retrospectively by means of clinical examination, device-based assessment (Cutometer ${ }^{\circledR}$ MPA 580, DSM II ColorMeter $\left.{ }^{\circledR}\right)$ and by validated patient and observer questionnaires (VSS, POSAS). In addition, donor sites were evaluated by means of POSAS and medical complications and subjective complaints were recorded. Statistical analysis was performed by means of Welch unpaired T-Tests.

Results: Glabrous grafts from the foot showed significantly superior results in terms of the ColorMeter (erythema score $5.73 \pm 2.64$ for group 1, $2.33 \pm 1.97$ for group 2; pigmentation score $9.82 \pm$ 5.42 for group 1, $1.89 \pm 1.92$ for group 2, p < 0.001). Evaluation of the scar by the observer by means of VSS and POSAS showed significantly superior results for almost all single items, total score and overall opinion. Conversely, FTSGs were significantly less stiff as measured by the Cutometer (mean normalized tissue extension R0 was $0.80 \pm 0.21$ for group $1 ; 0.57 \pm 0.24$ for group2; $\mathrm{p}<0.05$ ). Range of motion and assessment of sensitivity was inconclusive due to the heterogeneity of the scars and patient incompliance due to young age.

Conclusion: STSG from the plantar foot lead to satisfying results in terms of function and aesthetics in palmar paediatric burns. However, joint-crossing injuries of the digits might benefit more from FTSG. 
O1.06 Establishment and Effects of a Method for Wound Repair in Patients with Extensive Deep Burns Using Fresh Skin Allografts and Autologous Micrograft

\section{CA Shen}

The Fourth Medical Center of Chinese People's Liberation Army General Hospital, BEIJING, China

Objectives: This study attempted to establish a method for wound repair in patients with extensive deep burns using fresh allogeneic scalp combined with autologous micrograft.

Methods: Two patients with burn injuries involving 90\% (third-degree, 70\%) and 97\% (thirddegree, $85 \%$ ) total body surface area (TBSA) respectively were treated with fresh scalp allografts donated by 32 males aged $(31.5 \pm 8.2)$ years or autologous micrografting. The bilateral limbs with third-degree burns were selected as treatment and control groups. Wounds in the treatment group were treated with fresh allogeneic scalp and autologous micrograft, while wound in the control group received MEEK grafting. Preoperatively, the surgical area on the extremities was calculated to estimate the necessary amount of allogeneic scalp and MEEK grafts. Fresh scalps $(0.30-0.35 \mathrm{~mm})$ were harvested from each donor to prepare a larger piece of skin allograft. Autologous micrografts were transported onto the epidermis of the skin allograft. The treatment and control group received grafting according to our protocol. The donors received follow-up visits after 3 months to see if there is alopecia and scar hypertrophy. The wound coverage rate was observed in both treatment and control groups on postoperative weeks 2, 3, 4 and 5.

Results: The donor sites in all allogeneic skin donors healed within 10 days postoperatively. The scalp recovered well without any alopecia or scar hypertrophy during the follow-up visits. The wound coverage rate of the treatment group was approximate to or higher than that of the control group.

Conclusion: Considering that allogeneic skin is scarce and expensive and the patient's relatives are willing to help save the patient's life by donating the scalp, this method may be a feasible option in clinical practice.

O2.01 Decontamination or Irrigation? Effect of Initial Irrigation on Severity of Eye Burns Comparison of Two Rinsing Protocols Based on a 30 Years Ran Database

DU Uthoff $^{1}$, NW Wiesner ${ }^{2}$, NS Schrage ${ }^{1}$

${ }^{1}$ Eye Hospital Merheim Cologne, COLOGNE, Germany

${ }^{2}$ St. Augustinus Hospital Dueren, DUEREN, Germany

Objectives: Burns on the eyes are caused by various chemical agents. Concepts of irrigation divise into removal by flushing, dilution and/or decontamination of chemical action of caustic substances. To elucidate the clinical effect of rinsing versus decontamination we evaluated we used data from our eye burns database. The aim of this evaluation is to compare two rinse protocols based on the initial rinse with their effect on the resulting corrosivity grade.

Methods: Retrospective evaluation of a database. The survey was conducted continuously in two specialized eye clinics, with an evaluation cohort on the reference date 01.04.2018 of $n=1495$. Compared were protocol A with a primary rinse with Ringer lactate (1988 to 2006) and protocol B with a primary rinse with Previn (2006 to April 2018). The data were collected at the University of Aachen until 2004. From 2004 until today at the hospital Merheim in Cologne. We compared the clinical results in grading of eye burns using the Roper Hall classification in dependency of the used protocol.

Results: On average, 66.1 burned persons were treated per year until 2006 and 65.5 persons per year since 2006. An irrigation with an isotonic electrolyte solution resulted in a corrosivity of Grade 0 to 2 in $50.6 \%$ patients and the flushing with Previn ${ }^{\circledR}$ in $63.6 \%$ patients. This corresponds to a relative risk reduction of severe non-healing corrosivity grades Grade 3 and 4 by $79 \%$.

Conclusion: The amphoteric rinse solution leads to a clear reduction of severe eye burns. Especially in regard of a simplified interdisciplinary primary emergency care, the availability of a multivalent amphoteric rinsing solution in initial irrigation is a purposeful simplification for the 
emergency care. Our aim is reached with a clear benefit for the patient if we use Protocol B with initial Previn rinsing.

O2.02 Epidemiology of Fire-Related Injuries Requiring in Patient Care in Finland, 2002-2017

\section{KM Haikonen}

National Institute for Health and Welfare, HELSINKI, Finland

Objectives: The aim of this study was to assess the epidemiology of fire-related injuries leading to inpatient care in Finland.

Methods: Injury database (FINJURY) of the National Institute for Health and Welfare in Finland was utilized to identify patients with fire-related injuries that required inpatient care. The database captures all injury-related care periods recorded in the Finnish National Hospital Discharge Register which practically covers all inpatient care taking place in Finland. Fire injury admissions were captured by ICD-10 classification of external cause of injury used in the data. The latest year of data was 2017. Therefore, our assessment covers a period of 1.1.2002 - 31.12.2017. Basic statistical methods were used; Poisson regression for trends, t-test for two-group comparisons and Chi-square test for categories.

Results: A total of 4593 patients with fire-related injuries were observed during the period of 2002-2017 yielding an annual average of 287 cases (53.6 per 1 million in population). Seventy-two percent of the patients were male. Mean age of patients was 46.6 years (43.9 for males and 53.4 for females). The majority of cases were accidental but some $4 \%$ were self-inflicted. Among all firerelated injuries, a slightly decreasing trend was observed. However, a significant decreasing trend was only observed among males while for females there was no trend. Fire-related injuries with a burn injury decreased significantly during the study period. On the other hand, fire-related injuries with combustion gas poisoning significantly increased during the period.

Conclusion: Fire-related injuries cause a significant burden to society as well as significant suffering for the victims. Even though there has been positive progress with decreasing trend among fire-related burns, the increase in combustion gas poisonings is alarming. Efforts should be put into planning and implementing appropriate preventive measures.

\section{O2.03 Long-Term Outcome after Renal Replacement Therapy in Severe Burns}

IR Rakkolainen

Helsinki University Hospital, HELSINKI, Finland

Objectives: Acute kidney injury (AKI) is a common adverse sequela after major burn injury. The incidence of AKI is notably greater than the need for renal replacement therapy (RRT) after severe burn injury. In the vast majority of patients, renal function recovers before discharge from the burn center; there is limited literature on long-term outcomes. A few population-based register studies report an increased risk for chronic renal failure after burn injury.

The purpose of this study was to investigate the long-term outcome of severely burned patients that received RRT during acute burn injury treatment.

Methods: Data on 68 severely burned patients that received RRT in Helsinki Burn Centre between November 1988 and December 2015 were collected retrospectively. Thirty-two patients survived and remained for follow up after the primary hospital stay until 31 December 2016.

Results: The one-year mortality after discharge from the burn unit was $18.8 \%$ and $56.3 \%$ of discharged patients were alive at the end of follow up (average follow-up time 7.4 years; range 57622 days). In $81.3 \%$ of discharged patients, renal function recovered before discharge. Two patients received RRT for longer than three months, however, renal function of both patients recovered after 10.5 and 17 months, respectively. During follow up, $36 \%$ of deaths occurred due to burns, $7 \%$ due to kidney failure, and $57 \%$ for other reasons. One patient required chronic dialysis a few years after kidney function recovery.

Conclusion: A long-term need for RRT after severe burn injury is rare. In the vast majority of patients, renal function recovers before discharge from primary care. No causality between severe 
burn injury and chronic dialysis was observed. However, severe burn injury may potentially worsen underlying weakened kidney function in susceptible individuals.

O2.04 Establishing Disability Weights and The Burden of Burn Injuries in Australia, New Zealand and The Netherlands

I Spronk $^{1}$, D.W. Edgar ${ }^{2}$, M.E. Van Baar ${ }^{1}$, F Wood $^{3}$, N.E.E. Van Loey ${ }^{1}$, E Middelkoop ${ }^{1}$, B Renneberg ${ }^{4}$, C Öster ${ }^{5}$, L Orwelius ${ }^{6}$, A.L. Moi' ${ }^{7}$, S Polinder $^{8}$, J.A. Haagsma ${ }^{8}$

${ }^{1}$ Association of Dutch Burn Centres, ROTTERDAM, Netherlands

${ }^{2}$ Royal Perth Hospital, Burn Service of Western Australia, PERTH, Australia

${ }^{3}$ University of Western Australia, PERTH, Australia

${ }^{4}$ Freie Universitaet Berlin, BERLIN, Germany

${ }^{5}$ Uppsala University, UPPSALA, Sweden

6University Hospital Linköping, LINKÖPING, Sweden

7Western Norway University of Applied Science, BERGEN, Norway

${ }^{8}$ Erasmus MC, University Medical Center Rotterdam, Department of Public Health, ROTTERDAM, Netherlands

Objectives: Burden of disease calculations are an important resource in public health. An essential factor for burden of disease calculations is the disability weight, which reflects the magnitude of health loss associated with outcome and ranges from 0 (full health) to 1 (death). Currently, only rough estimates are available for the burden of burns due to the lack of disability weights for detailed and homogeneous burn injury groups. Our objective is to elaborate on a refined methodology to derive disability weights for homogenous burn injury groups, and to use this methodology to calculate the burden of burns of Australia, New Zealand and the Netherlands.

Methods: Australian and European generic health-related quality of life (HRQL) data were used to derive disability weights for three homogenous burn injury groups based on\%TBSA burned. Subsequently, incidence data from Australia, New Zealand and the Netherlands were used to compute years lived with disability (YLDs), which expresses the non-fatal burden of disease of burns.

Results: The disability weights, derived from HRQL data of 1687 European patients, ranged from 0.05 (subgroup $<5 \%$ TBSA burned; $>24$ months post-burn) to 0.58 (subgroup $>20 \%$ TBSA burned; 0-1 months post-burn). Incidence data from Australia, New Zealand and the Netherlands will be used to estimate the non-fatal burden of disease by country, sex, age group and burn injury group. This project will be conducted in April - June 2019, and final results, including disability weights based on Australian HRQL data, will be presented at the EBA 2019.

Conclusion: This project will lead to improvement of calculations of the burden of burn injuries by more precise estimates of disability after burn injuries. The improved method can be applied internationally. Burden of burns information can be used as input for health decision making at both the national and international level.

O2.05 The Course of Health Related Quality of Life after Burn Injuries Over Time: A Secondary Data-Analysis

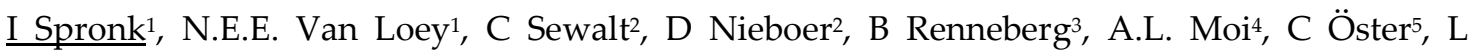
Orwelius ${ }^{6}$, M.E. Van Baar ${ }^{1}, \mathrm{~S}$ Polinder ${ }^{2}$

${ }^{1}$ Association of Dutch Burn Centres, ROTTERDAM, Netherlands

2Erasmus MC, University Medical Center Rotterdam, Department of Public Health, ROTTERDAM, Netherlands

${ }^{3}$ Freie Universitaet Berlin, BERLIN, Germany

${ }^{4}$ Western Norway University of Applied Science, BERGEN, Norway

${ }^{5}$ Uppsala University, UPPSALA, Sweden

6University Hospital Linköping, LINKÖPING, Sweden

Objectives: A prominent outcome measure within burn care is health related quality of life (HRQL). Up till now, the long-term detailed course of HRQL in adult burn patients was not yet studied due to limited availability of long-term longitudinal HRQL data. Re-use of existing data can 
overcome this issue. The aim of this secondary data analysis was to assess how HRQL after a burn injury evolves over time.

Methods: Generic HRQL measured in adult burn patients (either measured with the EQ-5D or SF-36) was requested from eleven European studies. SF-36 data was transformed into EQ-5D data to combine all datasets. EQ-5D utility scores over time were modeled using a linear mixed-effects model.

Results: HRQL data of 1687 patients a mean age of 42.5 (SD 14.9) years and a mean\%TBSA burned of $13.4 \%$ (SD 13.6\%) from ten European studies was used. EQ-5D utility scores from the different studies varied widely. The developed model showed large improvement in HRQL up to six months after burns, a steady improvement up to 18 months, and stabilization of HRQL thereafter. Females and patients with major burns have a delayed and worse recovery compared to males and those with minor burns. Improvements over time in the different dimensions of the EQ-5D were seen, except for the proportion of patients experiencing problems with anxiety/depression. Burn patients did not reach the levels of outcome of the general population in the dimensions pain/discomfort and anxiety/depression.

Conclusion: The developed model can be used in clinical practice to inform patients on expected outcomes, as well as for tracking of HRQL outcomes in adults with burn injury. In this way, a delayed recovery can be recognized in an early stage and timely interventions can be started in order to improve patient outcomes.

O2.06 The Long Term Results of the Collagen-Elastin Matrix Use in Single-Step Wound Closure Technique of Severe Full-Thickness Facial Burns in 12 Children

\section{Mehmet}

Inönü University School of Medicine, MALATYA, Turkey

Objectives: Management of full-thickness facial burns remains one of the greatest challenges. Controversy exists among surgeons regarding the use of early excision for facial burns. Unfortunately, delayed excision of deeper burns often results in more scarring and subsequent reconstruction becomes more difficult.

A collagen-elastin matrix is used to improve the quality of the reconstructed skin, to reduce scarring and to prevent wound contraction. It serves as a foundation for split thickness skin graft and enhances short and long-term results.

Our preliminary early results in single-step wound closure procedure of full-thickness facial burns in 15 children by using the collagen-elastin matrix was published in 2015.

By this presentation, the long term results of the usage of a collagen-elastin matrix during singlestep wound closure technique of severe full-thickness facial burns in our 12 children was reported.

Methods: Twelve pediatric patients with severe full-thickness facial burns who reported previously regarding early results were evaluated in according to long term results at the end of 5 years follow-up. Three of 15 patients have not been reached for this evaluation. The facial burn surface area (FBSA) among the patients had got in 7 patients with $100 \%$ and in five with $75 \%$. All patients had been treated by the simultaneous application of the collagen-elastin matrix (MatriDerm ${ }^{\circledR}$ ) and an unmeshed split thickness skin graft before 2015. The average Vancouver scar scales (VSS) were measured.

Results: The VSS were found $2.11 \pm 1.09$.

Conclusion: The long-term results show that the simultaneous application of the collagenelastin matrix and an unmeshed split thickness skin graft in deep facial burns in children improve the quality of the reconstructed skin, reduce scarring and prevent wound contraction.

\section{O2.07 An Applicable Tool to Compare Workload Among Different Burn Centres}

\section{IA Abdelrahman, IS Steinvall, FS Sjoberg, ME Elmasry}

Linköping University, LINKOPING, Sweden

Objectives: The Linköping Burn Interventional Score has been used to calculate the charges for each burned patient since 1993. The treatment of burns is versatile, and depends on the depth and 
extension of the burn. This requires a flexible system to detect the actual differences in the care provided. The aim is to describe the model of burn care that we used to calculate the charges incurred during the acute phase until discharge, so it could be reproduced and applied in other burn centres, which would facilitate a future objective comparison of the expenses in burn care.

Methods: We included all patients admitted with burns during the period 2010-15. We analysed clinical and economic data from the daily burn scores during the acute phase of the burn until discharge from the burn centre.

Results: Burns caused by hot objects and electricity resulted in the highest charges/TBSA\%, while charges/day were similar for the different causes of injury. Flame burns resulted in the highest mean charges/admission, probably because they had the longest duration of stay. Mean charges/patient increased in a linear fashion among the different age groups. Total median charge/patient was US\$ 28199 for 696 patients admitted. The charges adjusted by TBSA\% and hospital stay varied among different centres, ranging from US\$ 391 to 9043 for charges/TBSA\% and US\$ 426 to 3783 for charges/day

Conclusion: Our intervention-based estimate of charges has proved to be a valid tool that is sensitive to the procedures that drive the costs of the care of burns such as large TBSA\%, intensive care, and operations. The burn score system could be reproduced easily in other burn centres worldwide and facilitate the comparison regardless of the differences in the currency and the economic circumstances.

O3.01 Exploring the Mechanism of Wound Healing in Rats of Different Ages Using a Simple Burn Model

\section{CA Shen}

The Fourth Medical Center of Chinese People's Liberation Army General Hospital, BEIJING, China

Objectives: We developed a simple and stable animal burn model attempting to explore the mechanism of wound healing in rats of different ages.

Methods: A self-made metal column, heated in a boiling water bath, was applied for different time periods to the lower back of rats of different ages in burn creation. Wounds were observed visibly at different time points post burning. Biopsies were obtained and examined at $72 \mathrm{~h}$ post burn to determine the depth of burns. In exploring the mechanism of wound healing in rats of different ages, we used primary antibodies including Anti-EGFR [EP38Y] (ab52894) and p75NTR (D4B3) XP® Rabbit mAb (CST8238) for immunofluorescence of the skin paraffin sections $(5 \mu \mathrm{m})$ obtained from normal rats and on day 3 post burn creation. And we measured the expression of CD271 and EGFR.

Results: The distribution of CD271 and epidermal stem cells showed no age-related changes $(P$ $>0.05)$, and the expression of EGFR decreased with age in normal rats $(P<0.001)$. Compared with normal rats, the content of CD271 and epidermal stem cells and the expression of EGFR increased in burned rats.

Conclusion: The number of CD271 and epidermal cells and the expression of EGFR increase during wound healing in rats, suggesting that CD271, epidermal cells and EGFR in epidermal stem cell membranes are associated with burn wound healing.

O3.02 Topical Steroid Treatment for Suppression of Granulation Tissue in Burns: Results of a European Survey

$\underline{\text { Y Shoham }}^{1}$, R Tsur ${ }^{1}$, Y Krieger ${ }^{1}$, E Silberstein ${ }^{1}$, T Ayzenberg', E Maor ${ }^{1}$, L Rosenberg'2, M Harats ${ }^{3}$, J Haik $^{3}$, J Goverman ${ }^{4}$

${ }^{1}$ Soroka University Medical Center, BEER SHEBA, Israel

${ }^{2}$ Meir Medical Center, KFAR SABA, Israel

${ }^{3}$ Sheba Medical Center, TEL HASHOMER, Israel

${ }^{4}$ Mass General Hospital, BOSTON, USA

Objectives: The presence of granulation tissue in burn wounds, especially hypergranulation, may negatively influence healing time and contribute to development of hypertrophic scarring and 
contractures. Topical corticosteroids have been reported to suppress the inflammatory response that contributes to the growth of granulation tissue however literature about this treatment in burn wounds is limited. The aim of this study was to explore trends in the use of topical corticosteroids for suppression of granulation tissue amongst burn care professionals.

Methods: Participants of the 17th European Burns Association Congress were asked to complete an anonymous questionnaire regarding their experience with the use of topical corticosteroids for suppression of granulation tissue in burns. The survey included questions regarding the responders' background and experience with the use of topical corticosteroids in wound and burn care, and questions related to the safety and efficacy of this treatment for those experienced with its use.

Results: Eighty-two questionnaires were completed by 61 physicians (74\%) and 21 nonphysicians (26\%) with an average of 13.7 years of experience in burn care, and an average of 300 burn patients treated per year. Sixty-three $(77 \%)$ were experienced in the use of topical steroids for suppression of granulation tissue in burns. All of those experienced in this use found the treatment to be safe and effective. Forty-four (70\%) estimated they treated up to 50 burn patients with topical steroids per year, and $19(30 \%)$ estimated $>50$ patients per year. Only 1 of the 63 experienced responders $(1.5 \%)$ witnessed a case of possible systemic side effects. Only 7 of the $63(11 \%)$ experienced responders stated they had witnessed infections in $<10 \%$ of the patients they treated with topical steroids.

Conclusion: The use of topical steroids for suppression of granulation tissue in burn care is surprisingly widespread and appears to be safe and effective.

\section{O3.03 Virtual Reality as Adjunctive Therapy for Acute Pain in Adults Undergoing Burn Wound} Care

$\underline{\text { MS Cepeda Diez }}{ }^{1}$, S.G. Fidel Kinori¹, A García Palacios², D García Castilla-López², C Moreno Ramos $^{1}$, JA Ramos-Quiroga ${ }^{1}$, JP Barret Nerín ${ }^{1}$

${ }^{1}$ University Hospital Vall d'Hebrón, BARCELONA, Spain

${ }^{2}$ Universitat Jaume I, CASTELLÓN, Spain

Objectives: Acute pain during wound healing in patients with burns is one of the most and disabling pain as describe in literature. If this pain is not treated, it can lead to complications such as chronic pain or psychopathological disorders. One of the most successful applications of Virtual Reality (VR) is its use as adjuvant distraction technique for the reduction of acute pain associated with medical and nursing procedures. To carry out a spanish pilot study, the first in Spain to our knowlegde. Feasibility, acceptability and efficacy of a VR as an analgesic adjuvant in adult patients with burns in Vall d'Hebrón Burns Unit is explored.

Methods: Twelve patients admitted to Vall d'Hebrón Hospital Burns Unit with a counterbalanced intra-subject design. Patients who meet the inclusion criteria will be assigned randomly to one of two experimental conditions: 1 ) (usual intervention during the cures, $\mathrm{IH})+($ usual intervention + virtual reality, VR); 2) (usual intervention + virtual reality, VR) + (usual intervention during the cures, IH). The results of this pilot study will serve to design a controlled clinical trial that will aim to test the effectiveness of the VR intervention with a wider number of participants. The virtual world to be used is SnowWorld: a virtual environment by Dr Hunter Hoffman's group in the United States.

Results: We are currently in the course of analysis of results after the closure of the recruitment phase.

Conclusion: The relevance of this project lies in improving the treatment of acute pain associated with wound healing procedures in burn inpatients, a population that experiences intense pain. The results of this pilot study will serve to design a controlled clinical trial that will aim to test the efficacy of the VR intervention with a larger number of participants and its correlation with psychopathological variables.

O3.04 'Off Label Use' of MEPITEL ONE ${ }^{\mathrm{TM}}$. Managing Fatty Tissue after Early Excision of Full Thickness Burns

A Kyriakopoulos, I. Manolakis, M. Petroulia, G. Christopoulos, G. Harkiolakis 
Evaggelismos Hospital of Athens, ATHENS, Greece

Objectives: Early excision is the gold standard in full thickness burn treatment. The revealed fatty tissue needs to be covered in a way so it promotes epithelisation with granulation tissue as soon as possible so to achieve a well vascularised bed for the skin grafts. We used a thin, pliable, transparent, perforated silicone sheet, MEPITEL ONE ${ }^{\mathrm{TM}}$ to cover the revealed tissue.

Methods: 5 patients were included to the protocol of the survey with an average full thickness burn $10 \%$ TBS. The patients were on a high protein diet to keep the total protein of the blood from 5 to 6. Good consultation and singed consensus of the patients were performed prior surgery because this use was an 'of label use' of the product. Consent was also taken from the ethical committee of the hospital.

Results: We excised the full thickness burn area onday 2 after the burn incident. We used MEPITEL ONETM to cover the revealed fatty tissue, and gauzes with povidone solution and boric acid. We changed the gauzes every 3-4 days (two changes per week) and MEPITEL ONETM once a week. The desired granulation tissue was first seen in day 7 th and in day 10th the fat was completely covered. The product was easy to use, and tissue was visible because of the transparency of the silicone sheet. Blood was coming out of the holes of the product. The patient's feeling was confortable without pain. Skin grafts were placed on the 11th day and covered again the same way, on the 17th day grafts were successfully taken.

Conclusion: MEPITEL ONE ${ }^{\mathrm{TM}}$ promotes granulation over fatty tissue on burns, is easy to use, patient friendly, prevents contamination because the area is visible while covered.

\section{O3.05 Validity of the FLIR ONE PRO Thermography Camera for Measuring Burn Wound Healing} Potential

M.E. Carrière, L.E.M. De Haas, M.E.H. Jaspers, A. Pijpe, K. Gardien, A. Meij-de Vries, P.P.M. Van Zuijlen

Red Cross Hospital, BEVERWIJK, Netherlands

Objectives: Accurate assessment of burn wound depth and associated healing potential is vital in determining the need for surgical treatment in burns. Infrared thermography non-invasively measures the temperature of the skin, thereby providing indirect information on the blood supply and healing potential of burned skin. The FLIR ONE thermography camera is a small, low-priced, hand-held device, which previously demonstrated a high reliability, but a moderate validity for measuring burn wound healing potential. Therefore, the aim of this study was to evaluate the validity of a newer version, the FLIR ONE PRO thermography camera, for measuring burn wound healing potential, compared to Laser Doppler Imaging (LDI) as a reference standard.

Methods: Thermal images and LDI scans were obtained from acute burn wounds between 48 hours and 5 days post-burn. Temperature differences between burned and non-burned skin $(\Delta \mathrm{T})$ were calculated. To evaluate validity, $\Delta \mathrm{T}$ values were compared to the healing potential categories assessed by LDI. Two receiver operating characteristic curves were created to illustrate the ability of the FLIR ONE PRO thermography camera to discriminate between HP categories. Two cut-off values were chosen to differentiate between healing potential categories.

Results: A total of 43 burn wounds in 32 patients were included. $\Delta \mathrm{T}$ cut-off values of $-0.05^{\circ} \mathrm{C}$ (sensitivity $80 \%$, specificity $79 \%$ ) and $-2.1^{\circ} \mathrm{C}$ (sensitivity $31 \%$, specificity $90 \%$ ) were selected to discriminate between healing potential categories $<14$ days $-\geq 14$ days, and $\leq 21$ days $->21$ days, respectively.

Conclusion: As this study shows a good validity of the feasible FLIR ONE PRO camera for the assessment of burn wound healing potential, we consider the FLIR ONE PRO thermography camera a valuable addition to clinical evaluation. In future research, we aim to create a validated color-coded map to enable fast en easy interpretation of FLIR ONE PRO thermal images by clinicians.

O3.06 Implantation of Collagen- Elastin Matrix Under Split-Thickness Skin Graft on Early Excised Burn Bed Increases the Survival Rate 
Inönü University School of Medicine, MALATYA, Turkey

Objectives: Survival rate of split-thickness skin graft (STSG) is one of the problems during the treatment of severe burns, and is affected some factors (graft bed, age, additional morbidities, wound area etc.).

Single-step procedure (SSP) which included early excision of wound, implantation of collagenelastin matrix (CEM) on wound and grafting by split thickness skin is the our routine for pediatric burns in particularly specific body area (on face, joints, hand and foot).

In this retrospective study, we compared the survival rates of STSG under which used and not used CEM.

Methods: The success rates of 72 STSGs applied to 32 children with mean $15 \%$ TBSA of third degree burns were evaluated retrospectively. There were 14 girls and 18 boys ranging in age from 1 to 12 years, mean age 6 years. Data were analyzed to compare outcomes of STSGs for two groups; 1 ) SSP group in which CEM (MatriDerm ${ }^{\circledR}$ ) applied under graft, and 2) only graft group in which not used CEM. The $t$ tests were used to analyze differences in results in SSP vs. only graft groups ( $<<0.05)$.

Results: Mean graft survival rates in SSP and only graft groups were $99 \%$ and $84 \%$, respectively.

Conclusion: Its means that implantation of CEM under STSG increases significantly the survival rate of the graft That's why; we should recommend applying CEM under graft in early excised wound bed to increase the success rate of STSGs. 
O4.01 Participation of Parents in Paediatric Wound Care: A Review of the Literature

AEE De Jong ${ }^{1}$, MR Egberts' ${ }^{2}$ HWC Hofland ${ }^{3}$, NEE Van Loey ${ }^{2}$

${ }^{1}$ Red Cross Hospital, BEVERWIJK, Netherlands

${ }^{2}$ Association of Dutch Burn Centres, BEVERWIJK, Netherlands

${ }^{3}$ Maasstad Hospital, ROTTERDAM, Netherlands

Objectives: Participation of parents during burn wound care is surrounded by controversy. To increase our understanding of this subject in order to improve quality of care for children and their families, the literature has been reviewed. Research questions were: How is parental participation described? What are advantages and disadvantages? Which factors are important for application in daily practice?

Methods: A literature search that included papers using a diversity of research designs was carried out. A search of the databases CINAHL, Cochrane Library, Embase, PsychInfo, PubMed and Scopus yielded 385 articles. Nine articles were selected for complete review. Papers were evaluated using the model of Whittemore and Knafl.

Results: A coherent description of participation during wound care was not found, but it showed to be a complex and multi-dimensional concept comprising factors related to child, parent and healthcare professional and ranges from allowing parents to be present to involvement in care. Reported advantages are maintenance of family relationships and parental roles, education of wound- and scar care, a decrease of distress, guilt and psycho-social sequelae, and an increase of perceived control. Described disadvantages are that parents find it difficult to see their child crying and in pain, it reminds of the accident, causes worries about scarring, and generates physical reactions and distress. A suggested consideration for practice is that participation is optional. Furthermore, recommendations include comprehensive information and preparation, defining the parental role, ability to participate and cope, need for support and guidance, and evaluation.

Conclusion: A preliminary description of participation includes presence and support in ways that parents determine to be helpful, as long as it is a choice, does not obstruct care and that tailored guidance is facilitated. Research should focus on further analysis of the concept and on the connection to a care protocol.

\section{O4.02 Improved Sedation During Wound Care in Children with Burns}

C Kooiman, EWI Hoekstra, JCB Anink, L Sipkens, AEE De Jong

Red Cross Hospital, BEVERWIJK, Netherlands

Objectives: Children with burns are exposed to daily painful wound care procedures. The Dutch Healthcare Quality Authority introduced the guideline 'Sedation and/or analgesia (PSA) outside the operation theatre' to improve quality and safety regarding sedation during medical procedures. A sedation specialist, educated and competent to apply PSA, has been introduced. This health care professional can apply all three stages of sedation (light, moderate and deep) and is supervised by an anaesthesiologist when the child is younger than five years old. Our aim is to describe our first experiences after the introduction of the SPS.

Methods: After PSA introduction, we use rectal or intravenous pain medication in combination with nitrous oxide and/or sevoflurane or propofol to induce moderate/deep sedation. During wound care, the sedation specialist regulates the medication based on the observed pain behaviour. The pain is assessed using the COMFORT-B, a pain behaviour observation scale with validated cut-off points for mild, moderate and severe pain.

Results: Since the introduction of PSA, 170 young children underwent a total of 530 procedures. Despite the disadvantages that some children need an IV line and may have a temporarily decreased nutritional state due to the nil per os policy, we feel that this innovation results in more comfort for the child and its family. Pain did not exceed mild pain and the duration of the procedures decreased, since in our experience the course is undisturbed and calm.

Conclusion: We assume that PSA contributes to more comfort during procedures in wound care. However, more attention is needed to anticipate parental needs during wound care and the child's nutritional state. Further research is needed to investigate long-term effects of PSA. 
O4.03 To See the Dressings Shrink- Critical Care Nurses Experiences with Using Diaries for Burn Patients

$\underline{\text { S Daltveit }}{ }^{1}$, L Holme 2 , GB Sæmundsdottir ${ }^{2}, \mathrm{AL} \mathrm{Moi}^{2}$

${ }^{1}$ Department of Anaesthesiology and Intensive Care, Haukeland University Hospital, BERGEN, Norway

2Department of Plastic, Hand and Reconstructive Surgery and National Burn Centre, BERGEN, Norway

Objectives: The objective of this study was to explore and describe intensive care nurses' experiences with writing diaries to burn patients. Available research indicates that disturbed memories and/or a lack of memories after an intensive care can contribute to the development of anxiety, depression and posttraumatic stress-syndrome (PTSD), and the aim of the diary is to answer some of the patient's questions regarding what happened during the intensive care stay. To the best of the authors knowledge, this is the first study exploring nurses' experiences writing diaries for burn patients.

Methods: This qualitative research study used a focus group interview methodology. Two focus group interviews, including in total eleven nurses and intensive care nurses working at a burn unit, where recorded, transcribed and analysed using thematic analysis.

Results: Three themes were found. "A coherent story" describes how the diary must present a continuous and coherent description of the patients` stay. "A visual story" underlines the importance of pictures. "Formulating the patient's story" reflect the nurses aim to write the diary to the patient, telling his story. The nurses wrote diaries based on a desire to create something that makes life after intensive care easier for the patients, and they regarded the diary as important. However, there were several challenges related to writing diaries, including lack of time, insecurities related to language use, and the balance between the personal and the private.

Conclusion: The nurses viewed the diary as an important tool for burn patients, and the aim of the diary was to help the patient in the time after intensive care. However, there is a need for an increased degree of integrating the use of diaries as a part of the unit's regular routines.

\section{O4.04 Early Pressure Scar Therapy}

VG Gut $^{1}$, SS Simone ${ }^{1}$, JP Plock ${ }^{1}$, PM Meier ${ }^{2}$

${ }^{1}$ University Hospital Zurich, ZURICH, Switzerland

${ }^{2}$ Rehaklinik Bellikon, BELLIKON, Switzerland

Objectives: Scars resulting from burn injuries may lead to significant functional and aesthetic limitations. Compression therapy is standard of care for scar management.

Methods: Due to profound fluid shifts, the use of customized compression garment is limited during the acute phase. We evaluated therefore early compression with a tubular bandage providing a temporary measure in addition to conventional wound dressing before definitive compression therapy. The tubular bandage can be applied as early as during the exsudative phase of wound healing. After each dressing change (usually $3 x /$ week), the tubular bandages are applied directly over the wound dressing. Scar management using early compression requires very close cooperation among nurses and physiotherapists.

Results: Based on known effects of compression therapy on wound healing, the use of elastic tubular bandages offers an early measure for scar compression with $10-14 \mathrm{mmHg}$ of pressure. The tube dressing method allows to fix the wound dressings in the acute phase with minimal use of bandages and without the need for patches. Furthermore the patients range of movement is improved which can also relieve itchiness and pain. With this method compression therapy can be introduced in a step-wise gradual manner.

Conclusion: The application of early compression proved to be an effective intervention, advantageous for scar maturation, improving comfort of the dressings and increasing adherence of the patients to compression garments. 
O4.05 Mechanical Cleansing of a Wound - Video Education

S Kalliomäki

HUS Jorvi hospital, ESPOO, Finland

Objectives: Helsinki Burn Centre and intensive care unit U2 cares for general adult patients requiring intensive care and burn patients in all phases of their treatment including intensive care, ward and rehabilitation as well as outpatient clinic. Our nurses are required to master many challenging areas of competence, wound care being one of them. Wound care and its teaching is an essential part of our job. One of the challenges is standardizing the teaching. The purpose of this presentation is to show our video education approach in wound teaching.

Methods: Literature review was used as a method to get knowledge from wound healing process, mechanical cleansing and the effectiveness of video education. The script of the video was planned based on that knowledge. Patient's written consent was acquired for filming and using the video material.

Results: According to the literature mechanical cleansing is an effective way to reduce biofilm and bacterial load from the wound and to help the process of wound healing. Nurses find video education positive because it is auditory and visual. It is easy to use and can be watched when needed.

Conclusion: Using video education to teach wound care is a useful way to learn wound care and to ensure standardization of teaching. A video can be watched when and where ever needed. A video is informative and also available in Youtube. It is a cost-effective way to teach practical procedures.

In the future video education could be used more widely when teaching nursing procedures.

O4.06 Ultrasonic-Assisted Wound Debridement in the Treatment of Complex Wounds

MJ Sanchez Garcia, ML Perez del Prado

ICS, BARCELONA, Spain

Objectives: Expose our professional experience with ultrasonic-assisted wound debridement (UAWD) in handling of complex burns and wounds. This therapy was used to remove bacterial biofilm, debride the injuries and promote the healing process, or as combined therapy before skin graft or vacuum assisted closure therapy.

Methods: We did a retrospective observational descriptive study between July 2018 and December 2018 to patients with complex wounds that receive ultrasonic technique. We reviewed clinical histories looking for macroscopic description of wound changes after the treatment. Furthermore, photos were taken before and after each session.

Results: The UAWD shows an effective and exhaustive biofilm and devitalised tissue removal. We observe preservation of healthy tissue and improvement of wound bed conditions. We found that the treatment time was reduced with an easy to use device, and the consequent impact on patients' physical, emotional and social wellbeing.

There were limitations in some patients' pain management, due to high analgesia requirements that would need to be assessed by anaesthetists.

Conclusion: UWAD plays an important role in the treatment of complex burns and wounds. Not only because it deeply cleans and debrides, but also provides the best wound conditions and tissue stimulation to promote a rapid and complete healing.

These new techniques reduce wound time treatment and raise nurses' quality care. Although there is a broad satisfaction, among patients and staff, in the use of UAWD, further research is recommended to improve its application and reduce limitations.

O4.07 The Occurrence of Itch after Burns: Is There a Need for Medication?

H.W.C. Hofland, H.J. Van Kempen

Maasstadziekenhuis, ROTTERDAM, Nederland

Objectives: Itching after burn injury is still poorly understood. Research has shown that itch intensity and frequency are not related to the size or depth of the burn injury, and can occur on the 
graft, on or around the burn or donor site. Itch can interfere with daily life and has been described as a severe problem after burn injury.

Methods: This study was conducted to descibe the occurrence of itch in all adult patients admitted to the burn centre Rotterdam in one month, for one year to identify the occurence of pruritus, prescribed medication and applied topical ointments. The Burn Itch Questionaire (BIQ) was used to record itch frequency and intensity.

Results: In total 35 patients were admitted ( $80 \%$ male). Most injuries $(70 \%)$ were induced by flame burns. Fifteen repondents (43\%) required an operation. TBSA varied from $0.5 \%$ up to $31 \%$. The first occurence of pruritus was 3 days after burn injury (range 3-25 days). The score varied from 3 or less in 24 respondents (69\%) to more than 8 in 1 respondent (3\%). After 3 and 6 months, $40 \%$ and $46 \%$ of the repondents were without pruritus, respectively. After 12 months $60 \%$ of the repondents did no longer have pruritus. Alhydran ${ }^{\circledR}$ (based on pure aloe vera) was used as topical agent. After 12 months $29 \%$ still applied Alhydran ${ }^{\circledR}$. Most respondents reported sharp reduction of itching after using this topical ointment. After 3, 6 and 12 months, only 6, 3 and 1 patient(s) still used antihistamins, respectively.

Conclusion: Pruritus is common after burns. Forty\% of the respondents experience itch after one year; medication is then rarely used. Respondents prefer to use topical ointments.

Since this study Alhydran ${ }^{\circledR}$ is not longer reimbursed by the insurance companies in the Netherlands. Other topical ointments are now used. It may be advisable to repeat this study.

O5.01 Pathogenesis of Hypertrophic Scar Formation in Children after Thermal Injury. Principles of Diagnosis and Treatment

O.V. Filippova ${ }^{1}$, I.N. Krasnogorskiy' ${ }^{2}$, A.G. Baindurashvili1', R.V. Vashetko ${ }^{3}$, K.A. Afonichev ${ }^{1}$

${ }^{1}$ The Turner Scientific Research Institute for Children's Orthopedics, SAINT-PETERSBURG, PUSHKIN, Russia

${ }^{2}$ The Turner Scientific Research Institute for Children's Orthopedics, SAINT-PETERSBURG, PUSHKIN, Russia

3Saint-Petersburg I.I. Dzhanelidze Research Institute of Emergency Medicine, SAINTPETERSBURG, Russia

Objectives: To optimize diagnosis and treatment of children with post-burn scars on the basis of a clinical and morphological study of scar tissue at various times of formation.

Methods: Anamnesis and clinical manifestations of postburn scars were analysed in 339 children. Resected scars and surpluses of skin autografts from 54 children (198 biopsies) were morphologically (histologically) studied with morphometric and statistical processing. Immunohistochemical investigation (using monoclonal antibodies) determined cells expressing antigens of: Mast Cell Tryptase/MCT/ and Chymase/MCC/; Macrophages CD68, cell apoptosis p53 and its inhibitor (bcl-2 protein); Transforming Growth Factor/TGF- $\beta /$; Proliferating Cell Nuclear Antigen/PCNA/; Matrix Metalloproteinases /MMP/ and their Tissue Inhibitors /TIMP/; markers of type I and type III collagen.

Results: All patients with hypertrophic scars had prolonged time (1,5-3 months) of wound healing. During the first months, the scar formation and its intensive growth accompanied with hyperemia, consolidation, burning pain, itch, trophic disorders (blisters, skin erosions and ulcers).

During the first 6 months of scar formation, significant changes were identified in scar tissue (in comparison with scars of later period and intact skin): increased number of lymphocytes, and cells expressing CD68, MCC, MCT, PCNA, TGF- $\beta$. In contrast to intact skin, the quantity of $p 53$-positive cells in scar decreased simultaneously with an increase of $b c l$-2-expressing cells. $M M P$-positive cell number decreased together with an increase of TIMP-expressing cells. Increased total area of blood vessel lumen, accompanied with a decrease in their number, later also significantly decreased.

Conclusion: Cell-regulatory imbalance of scar formation is most pronounced in the first 6 months, which indicates a significant prognostic role of this period. Considering this, we adhere to the following treatment principles: after burns and reconstructive surgeries, all patients are prescribed compression clothing, anti-inflammatory medicine and antihistamines up to 1 year; for 
patients with scar hypertrophy we prescribe collagenase. Erosion and scar ulcers are indications for surgical treatment.

O5.02 Efficient Isolation and High Yield of Epidermal Cells from Foreskin Biopsies by Dynamic Trypsinization

\section{CA Shen}

The Fourth Medical Center of Chinese People's Liberation Army General Hospital, BEIJING, China

Objectives: Cultured keratinocytes play important roles in burn wound healing and scientific research studies. We aimed to modify the isolation method to avoid over-digestion, maximize the number of isolated epidermal cells and establish a more efficient and innocuous way of cell isolation.

Methods: Compared to the conventional method, the modified method combines the more dynamic process of enzymatic digestion with multiple harvestings of dissociated cells via digestion. The cells from each harvesting were immediately re-suspended in culture medium with serum to avoid extended trypsinization and then pooled for further analysis.

Results: The number of viable cells isolated per gram of adult foreskin epidermis was $(18.88 \pm$ $13.22) \times 10^{6}$ cells in the control group and $(67.34 \pm 30.66) \times 10^{6}$ cells in the modified group $(P<0.001)$. No significant differences were observed in the proportion of CD49f-positive cells between the two groups $(P>0.05)$.

Conclusion: The modified method was significantly more efficient in dissociating keratinocytes from each unit of skin biopsy, which is particularly important for treating severe burns when donor skin is limited.

O5.03 Optimizing Delivery of Uncultured Keratinocytes by Spraying Keratinocytes in Combination with A Fibrin Sealant

J P Valtonen, J Vuola

Helsinki Burn Centre, HELSINKI, Finland

Objectives: Application of non-cultured keratinocytes is challenging as run off of cell suspension decreases immediate adherence of cells. Fibrin sealants have been used to enhance the adherence. Nevertheless runoff has been significant as sequential application of cells and fibrin sealants have been used. For few years we have been researching the possibility of combining keratinocytes with thrombin component of the commecial fibrin sealant (Artiss).

Methods: Immediate adherence was studied by using GFPHaCat cells sprayed on Petri dishes on planar and oblique planes $(0,30,60,85)$ either as cell suspension or within the fibrin sealant. To investigate cell viability and proliferation primary keratinocytes isolated from splitness skin grafts and mixed with thrombin were sprayed on collagen coated Petri dishes and cultured. The growth media was changed every other day. Scratch wounds were done with scalpel on day $8^{\text {th }}$ and the healing was followed for three weeks. Imaging was done with fluresence and lihtgmicroscopy.

Results: In microscopical analysis the immediate adherence of the GFPHacat cells sprayed within the fibrin sealant was higher than in control group in oblique planes. The isolated primary cells remained viable through the follow up and spread on Petri dishes. The scratch wounds made on day eight healed within 14 days.

Conclusion: Combining keratinocytes with thrombin component of fibrin sealant seems to enhance immediate adherence of the cells and decrease the run off. It does not seem to have negative effect on the viability or proliferation and so provides an optimized application method of the cells in vitro conditions.

Picture $1:$ https://www.eventure-online.com/parthenuploads/89/9EBA/add_1_541589_8ab8dec5-0905-442f-a51f-0c4138e6dd9c.png 
O5.04 Erosive-Ulcerative Lesions of Gastrointestinal Tract in Burned Patients

\section{A. Alekseev $^{1}$, A. Bobrovnikov' ${ }^{2}$ S. Popov ${ }^{3}$}

${ }^{1}$ A.V.Vishnevsky National Medical Research Center of Surgery ,, MOSCOW, Russia

${ }^{2}$ A.V.Vishnevsky National Medical Research Center of Surgery, MOSCOW, Russia

${ }^{3}$ Russian Medical Academy of continuous Postgraduate Education, MOSCOW, Russia

Objectives: To investigate incidence of erosive and ulcerative lesions of gastrointestinal tract (GI tract), including with gastrointestinal bleeding (GI bleeding), in burned patients.

Methods: The study is based on the analysis of treatment in 1338 burned patients. The changes in GI tract were diagnosed with aid of esophagogastroduodenoscopy (EGD). In 663 patients (group 2), $\mathrm{H}_{2}$-histamine receptor blockers were used for prophylaxis and/or antiulcer treatment; in 675 patients (group 2), proton-pump inhibitors were used.

Results: Erosive-ulcerative lesions of GI tract were noted in 180 (13.5\%) patients. Acute erosions of esophagus and stomach were observed in $27.5 \%$ of cases; acute erosions with ulcers of stomach and duodenum, in $72.5 \%$ of cases.

In patients with burn areas up to $30 \%$ of body surface, the incidence of such lesions was $5.2 \%$; in patients with burn areas more than $30 \%$ of body surface, the incidence was 6 times more, significantly increasing with deep burns more than $20 \%$ of body surface. The incidence of GI bleedings also depended on total burn area, and was 1.8 times more in patients with burn areas more than $30 \%$ of body surface.

Despite specific antiulcer therapy, erosive and ulcerative changes were observed in $10.5 \%$ of cases in group 1; in $42.8 \%$ of these cases, GI bleedings were noted. In patients of group 2, erosive aqnd ulcerative lesions were diagnosed in $16 \%$ of cases; however, on the background of preventive use of proton-pump inhibitors, these lesions were complicated by GI bleedings only in $28.7 \%$ of these cases.

Conclusion: Risk of GI lesions increases with increasing of burn severity. EGD in patients with burns more $30 \%$ of body surface allows to timely detect erosive and ulcerative lesions in GI tract, start specific treatment with use of proton-pump inhibitors, and reduce incidence of GI bleedings.

O5.05 Leclercia Adecarboxylata, Not So Rare in Burned Patients

Lailey $^{1}$, N Martin ${ }^{2}$

${ }^{1}$ St Andrew's Burn Service, CHELMSFORD, United Kingdom

${ }^{2}$ St Andrew's Burns Service, CHELMSFORD, United Kingdom

Objectives: Control of wound infection is essential to decreasing morbidity and mortality in severe burn victims. Routine microbial burn wound culture is vital to reducing the incidence of infections due to pathogens with antibiotic resistant profiles. Leclercia adecarboxylata has been described as a rare cause of disease in humans, with only one case reported in burns. The aim of this study is to determine the true prevalence of L. adecarboxylata and identify any high risk populations.

Methods: A retrospective search was carried out on Broomfield Hospital's microbiology database to identify the number of cases of bacteraemia caused by L. adecarboxylata between July 2013-July 2018. Location of the case in Broomfield hospital, antibiotic sensitivity and severity of burn wound was also assessed. A literature search on EMBASE and Medline was carried out to identify all reported cases of L. adecarboxylata.

Results: In Broomfield Hospital, Chelmsford, we identified 56 isolates over the five-year period, 46 of which were isolated in moderate to severe thermal burns. Literature search revealed 75 cases of L. adecarboxylata since its identification in 1986, of which some had resistant profiles.

Conclusion: This study has identified Leclercia adecarboxylata as a rising pathogen in burns. Skin barrier dysfunction in the presence of highly exudative burn wounds represents an ideal microbiological niche and cause for its presence in burns. In this population, periodic surveillance for monitoring antimicrobial resistance of L. adecarboxylata and international compliance with antibiotic stewardship is strongly advised to help lower morbidity and mortality rates in burn victims. 
O5.06 Enzymatic Debridement is Selectively Effective in Burn Wounds Depending on the Thermal Damage of the Collagen: An Ex Vivo Burn Wound Experiment

$\underline{\text { KAA Kwa }^{1}, \text { L Van Haasterecht }}{ }^{2}$, E Middelkoop ${ }^{3}$, RS Breederveld ${ }^{1}$, ML Groot ${ }^{4}$, PPM Van Zuijlen', BKHL Boekema ${ }^{3}$

${ }^{1}$ Red Cross Hospital, BEVERWIJK, Nederland

${ }^{2}$ Amsterdam UMC, Department of Plastic, Reconstructive and Hand Surgery, AMSTERDAM, Nederland

${ }^{3}$ Association of Dutch Burn Centers, BEVERWIJK, Nederland

${ }^{4}$ LaserLab Amsterdam, Department of Physics and Astronomy, VU Universiteit, AMSTERDAM, Nederland

Objectives: The treatment of burn wounds with enzymatic debridement using bromelain in our burn center has shown promising results. However, inadequate debridement occurred in some cases with low temperature burns. We hypothesized that bromelain is ineffective in burns in which collagen denaturation, which occurs at $>65^{\circ} \mathrm{C}$, has not taken place. Our objective was to assess whether there is a relationship between the denaturation of collagen and the ability of bromelain to debride acute scald burn wounds of different temperatures.

Methods: Scald burns were induced to ex vivo human skin samples of $1 \times 1 \mathrm{~cm}$ by water immersion in temperatures of $40^{\circ} \mathrm{C}, 50{ }^{\circ} \mathrm{C}, 60^{\circ} \mathrm{C}, 70^{\circ} \mathrm{C}$ and $100^{\circ} \mathrm{C}$ for twenty minutes. Denaturation of collagen was assessed using histology, with hematoxylin and eosin staining, picrosirius red staining and a fluorescently labelled collagen hybridizing peptide (CHP), and with second harmonic generation (SHG) microscopy. Burned samples and one control sample (room temperature) were weighed before and after application of enzymatic debridement to assess the efficacy of bromelain.

Results: Unfolding of collagen, loss of basket weave arrangement, and necrosis was seen in samples heated to $70{ }^{\circ} \mathrm{C}$ or higher. Evident $\mathrm{CHP}$ fluorescence, indicative of collagen denaturation, was seen in samples of $70^{\circ} \mathrm{C}$ and $100{ }^{\circ} \mathrm{C}$. SHG intensity, signifying intact collagen, was significantly lower in the $70{ }^{\circ} \mathrm{C}$ and $100{ }^{\circ} \mathrm{C}$ group $(\mathrm{p}<0.0001)$ compared to the other groups. After enzymatic debridement, a weight reduction of $80 \%$ was seen in the samples of $70{ }^{\circ} \mathrm{C}$ and $100{ }^{\circ} \mathrm{C}$, the other samples showed a reduction of $20 \%$.

Conclusion: The status of collagen denaturation in skin samples, which occurred between $60^{\circ} \mathrm{C}$ and $70^{\circ} \mathrm{C}$, is strongly correlated with the efficacy of enzymatic debridement. Therefore, enzymatic debridement with the use of bromelain of burn wounds caused by temperatures up to and including $60^{\circ} \mathrm{C}$ is ineffective.

O7.01 Application of Free Flaps in Multidisciplinary Surgical Treatment of Patients with Subfascial Deep Burns

$\underline{\text { S Shapovalov }}^{1}$, V Vavilov ${ }^{2}$, A Pleshkov'1, E Sukhoparova ${ }^{2}$, A Panov ${ }^{1}$, YU Yunusova ${ }^{1}$

${ }^{1}$ All-Russian Center for Emergency and Radiation Medicine by A.M. Nikiforova EMERC, ST. PETERSBURG, Russia

${ }_{2}^{2}$ Pavlov First Saint Petersburg State Medical University, ST. PETERSBURG, Russia

Objectives: To improve the results of surgical treatment of patients with extensive subfascial deep burns exposing the deep anatomical structures of the limbs.

Methods: For a combined burn injury with extensive subfascial soft tissue death followed by exposure of the neurovascular bundles and limb bone tissue, 3 patients underwent reconstructive surgery with transplantation of free flaps with microvascular technique. When planning the stage of transplantation of grafts with microvascular technique, MSCT-angiography was used to assess the safety and topography of the recipient vessels. To assess the blood flow in the transplanted tissue complexes, a non-invasive percutaneous oximetry method was used.

All the victims were male, received a combined injury in a traffic accident. The average age was $32 \pm 5.6$ years.

Results: In all cases, using a microvascular technique, a positive result was obtained when transplanting complex tissue complexes. MSCT angiography made it possible to accurately evaluate the recipient vessels and plan the microvascular stage. The method of non-invasive percutaneous 
oximetry apparatus allowed us to adequately assess the state of blood flow (perfusion) in the transplanted free flaps. All patients restored the function of the limbs and work capacity.

Conclusion: For the preservation of the lower extremities, in the surgical treatment of patients with extensive subfascial deep burns with exposure of bone structures, free skin grafting with free flaps with microvascular technique is uncontested.

Comprehensive multidisciplinary surgical treatment of victims with combined extensive subfascial burns is a difficult task, which can only be done in a multidisciplinary specialized hospital.

O7.02 Microvascular Free Tissue Transfer in the Management of Acute High Voltage Electrical Burns: The Vall d'Hebron University Hospital Experience

A Monte Soldado, V Andreu Solà, J.M. Collado Delfa, J Aguilera Sáez, J Serracanta Domènech, J.P. Barret Nerin

Vall d'Hebron University Hospital, BARCELONA, Spain

Objectives: To describe our experience in microsurgical reconstruction of extensive defects secondary to acute high voltage electrical burns.

Methods: Retrospective review of patients who suffered from electrical injuries and required acute reconstruction with microvascular flaps between 2009 and 2019 in Vall d'Hebron University Hospital (Barcelona). The patients were analyzed for epidemiological characteristics, burn location, flap types, recipient vessels and complications.

Results: We present 20 cases of microvascular flaps performed in 16 patients who suffered from high voltage electrical burns with severe tissue damage. All of them were men, with a mean age of 38.5 years. The TBSA ranged between $0.5 \%$ and $70 \%$.

The anatomical regions that needed reconstruction were lower extremities in 10 patients, upper extremities in 5 cases and craneofacial region in 2 cases.

We performed 6 different flaps according to the needs of the recipient areas; the most used was the Anterolateral Thigh flap (9 cases), followed by Latissimus Dorsi flap (4 cases).

We encountered 5 complications in 20 flaps, which included: 2 cases of infection, managed by surgical debridement and local and parenteral antimicrobial therapy, and 3 cases of total flap loss (2 of them occurring in the same patient, that was finally successfully treated with a third microvascular flap).

Conclusion: Management of patients suffering from high voltage electrical injuries poses unique challenges for the reconstructive surgeon due to the massive tissue and vascular destruction that some of these patients suffer. With the progress and refinement of microvascular techniques, it has become possible to solve critical problems in surgical treatment. Furthermore, the aesthetic and functional outcomes may be improved compared to other techniques and might avoid amputations. Despite the complexity of this injuries, we consider we have had a good flap survival rate, possibly due to an accurate patient, flap and recipient vessel selection following an exhaustive local debridement.

O7.03 A Prospective Evaluation of Spray Keratinocytes and Cultured Keratinocytes to Treat Large TBSA Injuries

R. Sood

Richard M. Fairbanks Burn Center at Eskenazi Health, INDIANAPOLIS, USA

Objectives: Over the last two decades, a decrease in mortality rates of burn patients can be attributed to continued advancements in burn resuscitation, intensive care, trauma care and nutritional support and early excision and coverage of the burn wound. However, patients with burns $>50 \%$ TBSA continue to pose specific challenges to the burn surgeon with regards to autologus coverage due to their lack of donor sites. In September of 2016, we changed our practice with large TBSA burns by incorporating the use of spray keratinocytes (SK) in conjunction with CEA. This study describes our experience using spray keratinocytes in this patient population. 
Methods: This was an IRB approved prospective uncontrolled observational study evaluating clinical outcomes following the use of SK as an adjunct for closure in the treatment of life-threatening burn wounds, in patients who lack adequate available skin to harvest for conventional grafting.

Results: Seven patients ranging from 3.9 to 61.8 years of age were treated with SK. TBSA ranged from $43 \%$ to $95 \%$ with a mean of $60 \%$. Collectively, 33 treatment sites and 15 donor sites were treated with SK, with a mean area of $2425.0 \mathrm{~cm}^{2}$ and $325.0 \mathrm{~cm}^{2}$, respectively. As part of the complete treatment regimen for these severely injured patients, four of seven also received CEA.

Healing data at 4 weeks post-SK treatment was available for 31 of the 33 treatment sites and indicates that $93.5 \%$ of the wounds treated had $\geq 95 \%$ re-epithelialization. Evaluation of donor site healing indicated that by week $1,86.7 \%$ achieved $\geq 95 \%$ re-epithelialization.

Conclusion: The use of SK has been successfully used as an adjunct to achieve earlier definitive wound closure for patients with extensive burn injuries allowing for a potentially decrease length of stay, decrease incidence of complication related to prolonged open wounds, and an earlier return to a homeostatic state.

O7.04 Transplantation of Fresh Scalp Allograft in Repairing Extensive Deep Burn Wounds in Children

CA Shen

The Fourth Medical Center of Chinese People's Liberation Army General Hospital, BEIJING, China

Objectives: It is difficult to treat pediatric extensive burns, which contribute to high mortality rates, partly because of the lack of large allogeneic skin to close wound in China. This paper tried to describe an innovative use of fresh scalp as thin split thickness skin allografts to cover the burn wounds of pediatric patients.

Methods: Fresh scalp allografts were harvested from voluntary donors who were patients' relatives. The median total burn area in the major burns was of $40 \%$ TBSA, in depth of deep second to third degree. The fresh scalp allografts were transplanted on the wounds post tangential excision or escharectomy in the way of mere fresh scalp allografts coverage or mixed coverage with autografts and fresh scalp allografts.

Results: All the patients survived without serious complications during the treatment period. The median healing time was 47 days; the average healing time of the donors' scalps was $(7.6 \pm 1.08)$ days with no scar formation, alopecia areata or folliculitis post operation.

Conclusion: The use of fresh scalp allografts in the treatment of pediatric major burns is an effective and feasible method in protecting wounds and promoting wound healing as well as in reducing scar formation in the donor sites of burned children. The high ratio of fresh scalp areas to pediatric burn wound areas ensures high efficiency of wound coverage; and healthy relative skin donors have more initiatives and favorable healing results.

O7.05 Indeterminate Depth Burns: How Important Is Laser Doppler Imaging in The Early Decision Making Process for Surgical Intervention?

I. Monstrey, H. Hoeksema, J. Verbelen, J. Robbens, J. Claes

Ghent University Hospital, GENT, Belgium

Objectives: Laser Doppler imaging (LDI) is universally considered as the most evidence-based method for objective burn depth assessment by exactly determining its healing potential. We aimed to analyze on how exactly LDI can be useful as to the early decision-making for surgery in patients with indeterminate burns.

Methods: A survey was performed among burn centers who have an LDI at their disposal to gain insight on the practical use of LDI. This was done by means of an extensive questionnaire with a total of 32 questions then followed by in-depth interviews.

Results: Of the 51 centers we approached, 42 responded (82\% response rate) of which 27 completed the questionnaire (53\%)., Eighty-eight percent of the participants scored LDI as mainly to almost completely accurate and based their final decision for treatment on the combination of LDI 
and clinical assessment. Seventy-two percent of the participants allow LDI to modify or even alter their clinical opinion.

Conclusion: The more experience a burn center has with LDI the more it is considered as an indispensable diagnostic tool. The results of this survey show that LDI considerably reduces the percentage of indeterminate depth burns. For this group of burns, expert opinion recommendations are provided to facilitate the decision-making process especially whether or not to operate.

O7.06 Penile Skin for Reconstruction of Eyelids in Acute Major Facial Burns

F Marlborough, A Chilinska, L Clarke, S Varma

NHS, NEWCASTLE UPON TYNE, United Kingdom

Objectives: Eyelid reconstruction is of critical importance in burns patients. Shortage or contracture of peri-orbital skin can lead to ectropion and risk of corneal exposure.

We present the surgical techniques and outcomes in using autologous foreskin and penile shaft full thickness skin grafts in the management of complex severe facial burns with sight threatening corneal exposure.

Methods: Full thickness foreskin and penile shaft skin was harvested by the plastic surgeon. The oculoplastic surgeon performed full tarsorraphy. The graft was placed onto the debrided upper and lower lid defects as a composite, and secured using a fibrin sealant and sutures. Penile shaft donor sites were resurfaced with SSG.

Results: Since 2013, 4 patients aged 21-50 (3 flame, 1 chemical burn) were treated with this technique. TBSA range was $25-85 \%$.

1 patient sustained severe chemical burns to corneas which required one eye to be enucleated prior to lid reconstruction.

Good graft take was achieved in all patients. Full cornea protection was provided in all cases with good eyelid position and function maintained once the tarsorrhaphies were divided in all 4 cases.

The patients have not had any significant issues with subsequent penile donor site healing or function.

Conclusion: This technique offers robust full thickness skin in patients with limited donor sites. Crucially, the whole peri-orbital subunit is reconstructed with one skin graft.

Skin grafting to each lid separately often results in contracture and cicatrix of the graft resulting in recurrent corneal exposure.

Combining this choice of donor site with prolonged extensive permanent tarsorraphy we have been able to preserve the eyelid conformation and avoid early complications of contracture and recurrent exposure.

Surgeons should consider this an option in males with extensive facial/eyelid burns and limited donor sites.

O7.07 Cutaneous Alkaline Burns Caused by Household Cleansers

D.O. Vagner, E.V. Zinoviev, V.V. Soloshenko

St. Petersburg Institute of Emergency Care named after I.I. Dzhanelidze, SAINT-PETERSBURG, Russia

Objectives: Household cleansers used for sewer blockage elimination are widely used in Russia. The active substances in such liquids are sodium hydroxide and potassium hydroxide. Unguarded use of such cleansers can cause severe alkaline burns. However, such cases are infrequent and therefore there is no standard surgical treatment. The purpose of this study was to improve surgical treatment of patients with alkaline burns caused by household cleansers

Methods: Retrospective study of 5 patients treated in the burn department in St. Petersburg for cutaneous alkaline burns.

Results: To facilitate drainage of damaging cleansing products from adipose tissue escharotomy with numerous incisions was used in all cases as early as possible. Following stabilization of the patient's state we carried out excision of full thickness burns without skin graft. Due tothe lack of 
objective criteriafor evaluating viability of the remaining tissues one-stage skin grafting was inexpedient. Temporary wound coating was applied with the use of hydrocolloid dressing or NPWTdressing. Only after cleansing the tissues and the formation of granulations, we restored the skin with mesh skin grafts.

Conclusion: Early fascial necrotomy and delayed plastic surgery with mesh skin grafts are the priority methods of surgical treatment of patients with alkaline skin burns caused by household cleaning products.

Picture 1: https://www.eventure-online.com/parthen-uploads/89/9EBA/add_539825_0dcf688f7253-4a93-84bb-1a329bf4ea64.2.jpg

Caption 1: Patients with alkaline burn cleanser 'Krot'

Picture 2: $\quad$ https://www.eventure-online.com/parthenuploads/89/9EBA/add_1_539825_0dcf688f-7253-4a93-84bb-1a329bf4ea64.1.jpg

Caption 2: Patients with alkaline burn cleanser 'Stok-Master'

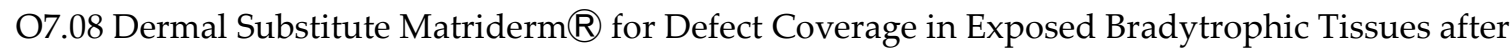
Severe Burn Injury

\section{M Öhlbauer, B Wallner}

BG Trauma Center Murnau, MURNAU, Germany

Objectives: Despite successful defect coverage by means of complex skin or muscle flaps, particularly large and deep problematic burn wounds with exposed bradytrophic tissues after soft tissue loss are very susceptible to surgical revision.

Matriderm $^{\circledR}$, a dermal substitute consisting of a native collagen matrix (collagen type I, III and V) supplemented by an elastin hydolyzate was first used for the treatment of burns, predominantly those that were full-thickness. Subsequently, its use was extended to defect coverage after soft tissue loss caused by fourth-degree burns.

Methods: 25 consecutive patients with exposed bradytrophic tissues caused by severe burn injury were treated the same way.

In all patients operative debridement showed soft tissue loss with free bone or free periostal structures, exposed tendons or joint capsules.

In all patients after accurate wound bed preparation defect coverage was performed with dermal substitute Matriderm ${ }^{\circledR}$ and unmeshed split skin grafts in combination with negative pressure wound therapy for one week for fixation of the split skin grafts.

Results: Two-years follow up of the Matriderm ${ }^{\circledR}$ procedure in defect coverage showed an excellent functional outcome:

Up until now, no areas with unstable scars have occurred, no surgical scar revision were required. The patients were still able to wear normal footwear, clinical gait analysis showed perfect functional outcome.

Conclusion: The application of dermal substitute Matriderm ${ }^{\circledR}$ in patients with exposed bradytrophic tissues after severe burn injury treated so far represents an excellent reconstruction method, from initial coverage to scar development.

O8.01 Procalcitonin Kinetics After Burn Injury and Burn Surgery in Septic and Non-Septic Patients A Retrospective Observational Study

G Tomé1, J Baltazar ${ }^{1}$, AR Meireles ${ }^{1}$, VM Afreixo², JL Cabral ${ }^{1}$

${ }^{1}$ Coimbra University Hospital Centre (CHUC), COIMBRA, Portugal

${ }^{2}$ University of Aveiro, AVEIRO, Portugal

Objectives: The present study evaluated procalcitonin (PCT) kinetics during the first days after burn injury and subsequent surgical interventions to assess PCT utility in distinguishing septic from nonseptic inflammatory responses.

Methods: This was a retrospective observational study of all burn patients admitted to the Coimbra Burns Unit (Portugal) between January 2011 and December 2014 who presented with a total burn surface area $\geq 15 \%$ and who underwent subsequent surgery. PCT kinetics were investigated a) 
during the first five days after burn injury and b) preoperatively and during the five days after surgery in three subsets of patients, including those with no preoperative and no postoperative sepsis $(\mathrm{NN})$, no preoperative but postoperative sepsis (NS), and preoperative and postoperative sepsis (SS). A total of 145 patients met the selection criteria and were included in the analysis.

Results: PCT levels in the first five days after burn injury were significantly higher in patients who developed at least one sepsis episode $(n=60)$ compared with patients who did not develop sepsis $(n=85)$. PCT values $>1.00 \mathrm{ng} / \mathrm{mL}$ were clearly associated with sepsis. Study participants $(\mathrm{n}=$ 145) underwent a total of 283 surgical interventions. Their distribution by preoperative/postoperative sepsis status was $142(50.2 \%)$ in NN; 62 (21.9\%) in NS; and 79 (27.9\%) in SS. PCT values exhibited a parallel course in the three groups that peaked on the second postoperative day and returned to preoperative levels on the third day or later. The lowest PCT values were found in NN, and the highest values were observed in SS; the NS values were intermediate.

Conclusion: PCT kinetics coupled with a clinical examination may be helpful for sepsis diagnosis during the first days after burn injury and burn surgery.

Picture $1:$ https://www.eventure-online.com/parthenuploads/89/9EBA/add_1_546068_32372d43-0a08-4028-b720-f0a54b577a22.jpg

Caption 1: ROC Curves for the discriminatory power of PCT levels between septic and nonseptic patients preoperatively and in the five days after burn surgery

Picture 2: https://www.eventure-online.com/parthenuploads/89/9EBA/add_2_546068_32372d43-0a08-4028-b720-f0a54b577a22.jpg

Caption 2: Median PCT levels observed from preoperative day (D0) till the fifth postoperative day (D5) for NN, NS and SS groups

Picture 3: https://www.eventure-online.com/parthen-uploads/89/9EBA/add_3_546068_32372d430a08-4028-b720-f0a54b577a22.jpg

Caption 3: Study population

Picture 4: https://www.eventure-online.com/parthen-uploads/89/9EBA/add_546068_32372d430a08-4028-b720-f0a54b577a22.jpg

Caption 4: Statistical analysis of PCT kinetics from preoperative day (D0) till the fifth postoperative day (D5) for NN, NS and SS groups

\section{O8.02 Ketamine-Related Sclerosing Cholangitis in Severe Burns}

M Raineau, N Donat, C Hoffmann, A Cirodde, JV Schaal, Y Masson, T Leclerc

Percy Military teaching hospital, CLAMART, France

Objectives: Hepatic disorders have increased in our burn intensive care unit (BICU), resulting in cases of "ischemic sclerosing cholangitis" (ISC) with worst cases resulting in four liver transplants from 2013 on. Concomitantly, ketamine (K) use, considered innocuous and with a broad safety range, increased as co-analgesic, then as hypnotic when concern about ischemia increased. Based on scarce literature reporting K abuse-related ISCs (Wong GL et al., Clin Gastroenterol Hepatol 2014), we finally restricted $\mathrm{K}$ use. We hypothesized that hepatic disorders were related to $\mathrm{K}$ exposure in our BICU.

Methods: All patients admitted to our BICU were reviewed in a retrospective study from 01/01/2007 to 30/06/2018 with demographic data, burn/injury severity scores, and hepatic biochemistry. Annual K consumption, averaged per patient, was used as a population exposure index. Three periods were compared: P1 (2007-2013) as historical period, P2 (2013-2017) from 1st ISC case on, and P3 (2017-2018) with implemented K restriction policy. Comparisons used ANOVA, Kruskal-Wallis or Wald tests as approriate.

Results: 1640 patients were included. Patients were younger but more severely burnt in P1, with higher general severity in P3 (tab.). K exposure estimated from annual department consumption was higher in P2 than in P1-P3 ( $\mathrm{p}<0,01)$. Hepatic disorders significantly differed between periods (tab. \& fig.), and matched K use. No ISC and limited hepatic disorders occurred in P3.

Conclusion: ISCs appeared related to K over-exposure in our BICU. Burnt patients are at known risk of liver dysfunction (Jeschke MG, in Herndon DN, Total Burn Care 4th ed.), but K related liver 
injury also likely occurred. Its not clearly understood mechanisms may involve a cumulative dose effect. Although concomitant involvement of other medications is being investigated, $\mathrm{K}$ restriction policy seemed to contain hepatic disorders.

Picture $\quad 1: \quad$ https://www.eventure-online.com/parthenuploads/89/9EBA/add_1_540980_ac48576c-589f-438c-abeb-17bf9a962926.PNG

Caption 1: Patient characteristics, outcome and biomarkers of cholestasis (2007 - 2018)

Picture 2: https://www.eventure-online.com/parthen-uploads/89/9EBA/add_540980_ac48576c589f-438c-abeb-17bf9a962926.PNG

Caption 2: Yearly average ketamine exposure and worst biomarkers of cholestasis

O8.03 A 5-Year Single-Centre Retrospective Study of Potential Drug Interactions in Burns Inpatients with Psychiatric Comorbidities

M Al-Khalili1, A Sack², R Elson², J Pleat ${ }^{2}$

${ }^{1}$ University of Bristol, BRISTOL, United Kingdom

${ }^{2}$ North Bristol NHS Trust, BRISTOL, United Kingdom

Objectives: To identify serious drug interactions in a group of burn inpatients who hypothetically were at elevated risk: those with psychiatric diagnoses. We aimed to identify the most common classes of drug involved and the potential clinical effects. Outcomes of interest were serious potential interactions between an existing medication and a new drug, or between two new drugs, and any clinical evidence of these interactions having occurred.

Methods: The International Burn Injury Database (IBID) was used to identify all admission episodes over a 5-year period at an adult regional burns unit for patients with a psychiatric comorbidity. For this group, all drugs administered (psychotropic and non-psychotropic) were categorised as either a new or continuing medication. Following this, a well-known interaction checking tool (Stockley's) was used to check for potential interactions and where one was identified, a retrospective notes review established whether there was any evidence of this having occurred clinically.

Results: Eighty-four admission episodes were identified from the IBID search and records were available for 60 of these. In total, 145 incidences of severe potential interactions were identified (89 between a new drug and a continuing drug and 56 between two new drugs).

The most frequently involved continuing drugs were neurotransmitter reuptake-inhibiting antidepressants and mirtazapine, while the most common new drugs identified were ondansetron, fentanyl and tramadol. The most frequently identified potential results of interactions were serotonin syndrome, arrhythmias and hypokalaemia. Clinically, there was minimal evidence for any interaction.

Conclusion: We have found many potential severe interactions in this patient group and psychotropic drugs were more commonly identified than other drug types. However, there was little evidence of the clinical manifestations of interaction. Serious drug interactions in burns patients are likely rare, but clinicians should be aware of the most likely drugs involved and the possible sequelae.

Picture 1: https://www.eventure-online.com/parthen-uploads/89/9EBA/add_535264_79c4b0dc52cf-4ed9-bfac-6ccf7e897e91.png

Caption 1: Serious potential interactions between a continued drug and a new drug: newly prescribed medication during admission ('new drug') most frequently impl

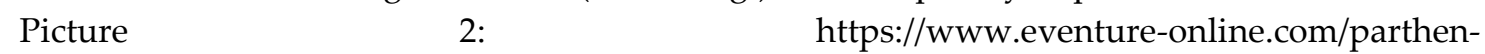
uploads/89/9EBA/add_2_535264_79c4b0dc-52cf-4ed9-bfac-6ccf7e897e91.png

Caption 2: The most frequently identified potential complications of interaction between a continued drug and a new drug

Picture 3: https://www.eventure-online.com/parthenuploads/89/9EBA/add_1_535264_79c4b0dc-52cf-4ed9-bfac-6ccf7e897e91.png

Caption 3: Serious potential interactions between a continued drug and a new drug: existing medication ('continued drug') most frequently implicated 
O8.04 Extracorporeal Membrane Oxygenation for ARDS in Burn Patients

M Dadras, JM Wagner, C Wallner, J Huber, D Buchwald, J Strauch, K Harati, N Kapalschinski, B Behr, M Lehnhardt

Berufsgenossenschaftliches Universitätsklinikum Bergmannsheil Bochum, BOCHUM, Germany

Objectives: Acute respiratory distress syndrome (ARDS) has a reported incidence of $34-43 \%$ in ventilated burn patients and is associated with a mortality of $59 \%$ in the severe form. The use and experience with ECMO in burn patients developing ARDS is still limited. We present our results and discuss the significance of ECMO in treating burn patients.

Methods: A retrospective analysis of burn patients treated with ECMO for ARDS between January 2017 and January 2019 was performed. Demographic, clinical and outcome data were collected and analyzed.

Results: 8 burn patients were treated at our institution with ECMO in the designated time period. Of these, all but one patient had inhalation injury, burn $\%$ TBSA was $37 \pm 23 \%$, ABSI score was $8,4 \pm 2$ and R-Baux-score was $98 \pm 21.7$ patients developed severe ARDS and one patient moderate ARDS according to the Berlin classification with a $\mathrm{PaO}_{2} / \mathrm{FiO}_{2}$ upon initiation of ECMO-therapy of $62 \pm 22$ mmHg. ECMO duration was $388 \pm 283$ hours. 3 patients died from severe sepsis while 5 patients survived to hospital discharge.

Conclusion: ECMO is a viable therapy option in burn patients developing severe ARDS and can contribute to survival rates similar to ECMO therapy in non burn-associated severe ARDS. Consequently, patients with severe respiratory insufficiency with unsuccessful conventional treatment and suspected worsening should be transferred to burn units with the possibility of ECMOtreatment to improve outcome and survival.

O8.05 Measuring Energy Expenditure Using Ventilator derived VCO2 in Severe Burns

S.C.J. Mul ${ }^{1}$, N. Vieleers ${ }^{1}$, A. Pijpe ${ }^{1}$, S.N. Stapel ${ }^{2}$, E. De Jong ${ }^{1}$

${ }^{1}$ Rode Kruis Ziekenhuis Beverwijk, BEVERWIJK, Nederland

${ }^{2}$ Amsterdam UMC, AMSTERDAM, Nederland

Objectives: In severe burns patients nutritional support is important due to the increased energy expenditure (EE) caused by the hypermetabolic state. Measurement of EE is recommended, however gold standard Indirect Calorimetry (IC) is not readily available and continuous measurement is unfeasible. Formula's to predict EE are validated but inaccurate. Ventilator derived carbon dioxide (VCO2) used to calculate energy expenditure (EE:VCO2) has proven to be accurate in other critically ill patients, however, evidence on the validity of this method in burn patients is lacking. We investigated the feasibility and validity of using $\mathrm{VCO} 2$ to calculate EE in severe burns.

Methods: This pilot study compared two methods of EE calculation in severe burn patients (adults, TBSA burned $>20 \%$, mechanically ventilated) to a golden standard Indirect Calorimetry (IC). The two methods were: EE:VCO2 calculating EE with a rewritten Weir formula and the currently used standard of care of formula based calculation of EE using the Harris and Benedict formula (EE:HB) adding 30\% for metabolic stress, plus the\%TBSA burned.

Results: Between 2016 and 2018 we included 10 patients with severe burns, median age 35 years (IQR 37), median TBSA 42.5\% (IQR 31.75). Results showed a systematic 20\% higher mean VCO2 measurement in the ventilator capnometer compared to the gold standard measurement. After adjustment for this difference, EE: $\mathrm{VCO} 2$ calculations correlated more strongly $(\mathrm{r}=0.840 ; \mathrm{p}=0.001)$ with IC compared to EE:HB ( $r=0.638 ; \mathrm{p}=0.026)$.

Conclusion: The results of this pilot study showed that the EE:VCO2 methods was feasible in severe burn patients. Additional research is necessary to investigate the superiority of the EE:VCO2 method over the EE:HB method in comparison to IC. A more accurate EE calculation method may improve the outcome of the severely burned. 
O8.06 Reliability of Resting Energy Expenditure in Major Burns: Comparison between Measured and Predictive Equations

D Kym

Hangang Sacred Hear Hospital, Hallym University Medical Center, SEOUL, South Korea

Objectives: Poor outcomes can result from inadequate energy intake. We aimed to investigate the reliability of resting energy expenditure (REE) measured by indirect calorimetry (IC) with REE calculated using predictive equations for nutritional support in patients with major burns.

Methods: REE was measured using IC and compared with predictive equations in 215 adult severe burns patients from Jan 2011 to Jun 2015. Agreement between IC and predictive equations was assessed using Bland-Altman methods.

Results: All predictive equations were compared with REE measured using IC. The mean measured REE was $1712 \mathrm{kcal} / \mathrm{d}$. Bland-Altman analysis showed that 1.2 times HBE, Thumb 25, and Ireton-Jones equations had higher accuracy and reliability. The concordance correlation coefficient was higher (0.49) in the Ireton-Jones equation, and root mean square error (RMSE) was lowest (471.5) in the Thumb 25 equation. The proportion of patients with predicted REE within $\pm 10 \%$ of measured REE was highest with Thumb 25 (52.5\%). Other equations for burns patients had higher mean bias and overestimated REE when compared with IC results.

Conclusion: This study suggests that Thumb 25 can be used as an alternative method for estimating energy requirements of patients with major burns when IC is not available or applicable.

\section{O8.07 Inhalation Injury Protocol in Finnish National Burn Centre}

MJ Hult, L Lindahl, TM Varpula

Helsinki University Hospital, ESPOO, Finland

Objectives: Inhalation injuries are related to increased morbidity and mortality among fire victims. Presence of inhalation injury verified by bronchoscopy has been shown to increase probability of death by $3.6 \%$. In our unit we have developed a protocol aimed to prevent respiratory complications related to smoke inhalation.

Methods: We performed a retrospective analysis of the patients in Finnish National Burn Centre (Jorvi U2) with fire related inhalation injury during 2016-2018. All patients with history of fire in enclosed space treated in our unit were screened. Those patients with clinical need of intubation were all examined with bronchofiberoscopy (FOB) according our guideline. Those patients with findings of soot in the trachea and or in the bronchioles were included in this study.

After finding of soot in lower respiratory track we performed FOB with small volume $\mathrm{NaCl}, 9 \%$ lavations followed with suctions in eye control. Procedure was repeated every 6 hours until soot was not seen in bronchioles. Medical therapy consisted of bronchodilators inhalation 4 times per day, corticosteroid inhalations twice per day and heparin inhalations (Heparin 5000ky) 4 times per day. Hydroxocobalamin $5 \mathrm{~g}$ iv was given by paramedics and repeated after 12-36 hours if patient had a lot of soot in bronchus and lactate level rises after FOB.

Results: Among 174 ICU burn patients during last three years 29 (16\%) had inhalation injury. Mean age was 56,6 and Baux-score was 94 . Mortality was $41.4 \%$. None of these patients developed a severe ARDS and gas exchange remained satisfactory without the need prone position or ECMO.

Conclusion: Early diagnostic and using of preventive protocol might be associated to low incidence of development of ARDS. Presence of inhalation injury was related to severe injuries. However mortality adjusted with Baux-score was comparable to reported in literature.

O9.01 Percutaneous Collagen Induction (Microneedling) in Non-Atrophic Scar Management: Literature Review

\section{Iosifidis ${ }^{1}$ I Goutos ${ }^{2}$}

${ }^{1}$ Barts and the London School of Medicine and Dentistry, LONDON, United Kingdom

${ }^{2}$ Queen Mary University of London, LONDON, United Kingdom 
Objectives: Percutaneous collagen induction (PCI) or needling techniques are increasingly popular in aesthetic practice. The underlying mechanisms of action rest on producing a pattern of non-ablative and non-confluent puncture wound pattern to the dermis with a resulting regenerative effect on the skin. The majority of publications in the scar literature focus on the use of PCI in the context of atrophic scars with no currently available summative reports appraising the pertinent evidence relevant to non-atrophic scars including burns.

Methods: A detailed English literature review was conducted using PubMed Medline, Embase and Web of Science; the manuscripts were appraised and classified according to the Joanna Briggs Institute Levels of Evidence. Results are presented in descending order of evidence.

Results: On the basis of level 1 evidence currently available, the combination of needling and silicone gel can improve the short-term pliability, height and vascularity of hypertrophic and keloid scars. According to level 2 evidence, needling alongside spray keratinocytes can produce a statistically significant improvement to patient/observer scar ratings and improve pigmentation in hypopigmented burn scars at 12-month follow up. Results from mixed cohort studies also point towards needling having a beneficial effect on fat graft retention. Level 3 data suggest that needling can render significant resurfacing effects to both mature and actively hypertrophic burn scars at 12month follow up based on objective scar scales; furthermore, favourable histological changes are seen including better collagen alignment in the dermis and thicker epidermal layer. A number of level 4 studies reinforce the promising role of needling in the resurfacing of both mature and actively hypertrophic scars.

Conclusion: Needling techniques are promising adjuncts to non-atrophic scar management. Further research with long-term follow up and comparative design protocols incorporating other resurfacing modalities is warranted before the exact value of needling is delineated in scar management protocols.

Picture $\quad 1: \quad$ https://www.eventure-online.com/parthenuploads/89/9EBA/add_1_539269_c5a92d34-4f1c-4d26-838a-dd981a2a5373..png

Caption 1: Flowchart for study selection

O9.02 Presence of Adipose Tissue after Autologous Fat Transplantation in Patients with Adherent Scars, A Pilot Study

R. Zuidema, S.J.M. Jongen, M.K. Nieuwenhuis, S.A. Van Ingen Schenau, L.S. Wagenaar

Martini Hospital, GRONINGEN, Netherlands

Objectives: A substantial number of burn survivors develop an adherent hypertrophic scar because the subcutis had to be excised. The important functions of the subcutis are therefore missing, as well as the functional sliding layer itself. These patients suffer from pain, itching, soft tissue or joint stiffness, as well as possible aesthetic and psychological consequences. Autologous fat transplantation (AFT) is an upcoming and promising technique for the reconstruction of the subcutis in adherent scars. Recent studies have shown a beneficial effect of AFT on the aforementioned symptoms. However, it is still not proven whether the transplanted adipose tissue is present and functional after a period of time, because not all adipose tissue might survive the grafting process due to surgical trauma or absence of blood supply. This study aims to assess the presence of subcutaneous adipose tissue after AFT using ultrasonography.

Methods: A prospective uncontrolled trial was carried out. Five patients with a total of seven adherent scars due to burns, necrotizing fasciitis or degloving injuries, undergoing AFT using the LipiVage method, were included. A subcutaneous ultrasonography was made preoperatively and at 3-months follow-up. These ultrasounds were assessed by a single experienced anesthesiologist.

Results: In five of the seven scars there was an increase in subcutaneous tissue visible (see figure 1 for a typical example). In one scar the result was inconclusive due to differences in ultrasonography angles and in one scar no increase in subcutaneous tissue was seen.

Conclusion: This study shows that adipose tissue is still present at 3 months follow-up after AFT and its presence supports the hypothesis that adipose tissue is instrumental in the treatment of adherent scars. 
Picture

ultrasound.png

Caption 1: Figure 1. Subcutaneous ultrasound: longitudinal posterior view of the proximal forearm, preoperative (left) and at 3-months follow-up (right).

O9.03 Patient Satisfaction Regarding Donor-Site Scars After Skin Graft Harvesting: A Prospective Cohort Study

CM Legemate $^{1}$, H. Goei ${ }^{2}$, ME Van Baar ${ }^{1}, \mathrm{CH}$ Van der Vlies ${ }^{1}$

${ }^{1}$ Maasstadziekenhuis, ROTTERDAM, Nederland

${ }^{2}$ Amsterdam UMC, AMSTERDAM, Nederland

Objectives: To investigate predictors for reduced satisfaction regarding scar quality of donorsites in burn patients and to create a predictive model to identify individuals at risk of poor satisfaction with the quality of their donor-site scars.

Methods: Between February 2017 and February 2018 all consecutive patients undergoing excision and skin grafting for their burn wound were included and prospectively followed in this cohort study. Scar quality of the donor sites was determined at three- and twelve-months postsurgery using the patient and observer scar assessment scale (POSAS).

Results: To assess the change in scar quality of donorsite scars over time, the 3 and 12 month results of the POSAS scores will be compared using descriptive statistics (means, SDs). With multivariable regression analysis the relationship between patient-, injury-, and treatment characteristics and the POSAS scores will be explored using the 3 and 12 month results that will be available at the time of the congress.

Conclusion: Results of this study enables us to provide better information to patients about what they can expect of the development of their donorsite scar and enables us to identify individuals at risk of poor donor-site scar satisfaction. In this way, we aim to provide better (patient-centred) clinical guidance and decision making.

O9.04 Development of the Patient and Observer Scar Assessment Scale 3.0

M.E. Carrière ${ }^{1}$, L.B. Mokkink ${ }^{2}$, A. Pijpe ${ }^{1}$, H.C.W. De Vet², P.P.M. Van Zuijlen ${ }^{1}$

${ }^{1}$ Red Cross Hospital, BEVERWIJK, Netherlands

${ }^{2}$ Amsterdam UMC, AMSTERDAM, Nederland

Objectives: Measurements of scar quality are essential to evaluate the effectiveness of scar treatments and to monitor scars over time. The Patient and Observer Scar Assessment Scale (POSAS) measures both the patient and professional perspective on scar quality. Since its introduction in 2004, it has been translated into multiple languages and is now widely used. However, during the initial development of the POSAS, the content was determined without the input of patients or international experts. Therefore, our goal is to develop the POSAS 3.0: an improved version for the assessment of scar quality in different types of scars, such as burn, necrotizing fasciitis, linear and keloid scars.

Methods: We have organized 5 focus groups in the Netherlands and 2 in Brisbane, Australia to explore which characteristics of scar quality matter the most to patients. To reach consensus among many scar experts on the content of the Observer Scale, we have performed an international Delphi study. Scar experts (i.e. Delphi panel members) were asked to fill in 3 different rounds of anonymous online questionnaires containing questions regarding the content of the Observer Scale.

Results: The results of these studies showed which characteristics of scar quality were considered to be most important by patients and professionals.

Conclusion: By including a broad range of scar experts in its development, we hope that the POSAS 3.0 will become a high-quality and widely used scar assessment scale that will not only improve the evaluation of treatment strategies, but also enhance the comparability of study results worldwide. 
O9.05 A Randomised Placebo-Controlled Trial on the effects of shockwave-therapy in burn scars older than six months.

$\underline{\text { M Anthonissen }}{ }^{1}$, P Moortgat ${ }^{2}$, J. Meirte ${ }^{3}$, C Lafaire ${ }^{4}$, L De Cuyper ${ }^{4}$, U Van Daele ${ }^{3}$, T Vanhullebusch $^{3}, \mathrm{~K}$ Maertens ${ }^{5}$

${ }^{1}$ Oscare - KULeuven, ANTWERP, Belgium

2Oscare, ANTWERP, Belgium

${ }^{3}$ Oscare - UAntwerpen, ANTWERP, Belgium

${ }^{4}$ Oscare - ZNA Stuivenberg, ANTWERP, Belgium

${ }^{5}$ Oscare - Vrije Universiteit Brussel, ANTWERP, Belgium

Objectives: A variety of physical treatments exists to manage hypertrophic scarring. One of these treatments is extracorporeal shockwave therapy (ESWT). Although the application of ESWT in scar management is still in its exploration phase, there are some interesting findings in the literature indicating its possible beneficial role in scar remodeling. The aim of this randomized, placebo controlled, double blinded trial was to investigate the effects of low- and high-intensity ESWT in patients with older burn scars.

Methods: Patients were randomly assigned to one of three groups and treated with pressure garments, silicone and moisturizers. Patients of group A and group B were additionally treated with respectively low-intensity ESWT $(0.25 \mathrm{~mJ} / 6 \mathrm{~Hz})$ and high-intensity ESWT $(0.76 \mathrm{~mJ} / 4 \mathrm{~Hz})$ once a week during 10 weeks. Patients of group $\mathrm{C}$ were treated with a placebo shockwave-probe.

The assessment methods were the Patient and Observer Scar Assessment Scale (POSAS) for clinical assessment, tristimulus colorimetry for redness, and cutometry for elasticity. Patients were tested at baseline, after one month, three months and six months.

For the statistical analysis, a mixed-design ANOVA was carried out, with the intervention being the between-subjects factor and time being the within-subjects factor.

Results: Preliminary results after six months on 30 patients are presented. Mean scar age varied between 7,6 months and 11,7 months. A trend towards a better performance of the low-intensity ESWT group was observed, although only the between-group differences for scar extensibility were statistically significant $\left(p=.045, \eta^{2} p=.243\right.$ ). The low-intensity ESWT group was also the only group that showed significant improvements over time for objective measurements of scar brightness $\left(\mathrm{p}=.002, \eta^{2} \mathrm{p}=.301\right)$, scar redness $\left(\mathrm{p}=.003, \eta^{2} \mathrm{p}=.495\right)$ and scar extensibility $\left(\mathrm{p}=.004, \eta^{2} \mathrm{p}=.388\right)$.

Conclusion: Low-intensity ESWT seems to have a benefit in the treatment of hypertrophic burn scars with an improvement of scar colour and extensibility.

O9.06 Patient-reported scar quality of adults after burn injuries: A five-year multicenter follow-up study.

I Spronk ${ }^{1}$, S Polinder², JA Haagsma², MK Nieuwenhuis ${ }^{3}$, A Pijpe ${ }^{4}, \mathrm{CH}$ Van der Vlies ${ }^{5}$, E Middelkoop ${ }^{6}$, ME Van Baar ${ }^{7}$

${ }^{1}$ Association of Dutch Burn Centres, Maastad Hospital, ROTTERDAM, Netherlands

2Erasmus MC, University Medical Center Rotterdam, ROTTERDAM, Nederland

${ }^{3}$ Association of Dutch Burn centres, Martini Hospital, GRONINGEN, Nederland

${ }^{4}$ Burn Centre, Red Cross Hospital, BEVERWIJK, Nederland

${ }^{5}$ Burn Centre, Maasstad Hospital, ROTTERDAM, Nederland

${ }^{6}$ Amsterdam UMC, Vrije Universiteit Amsterdam, AMSTERDAM, Nederland

${ }^{7}$ Association of Dutch Burn Centres, Maasstad Hospital, ROTTERDAM, Nederland

Objectives: Scar formation is an important adverse consequence of burns. Patient's appraisal of their scar quality is often studied shortly after sustaining the injury. However, information in the long-term is scarce. Our aim was therefore to evaluate long-term patient-reported quality of burn scars.

Methods: Adults with a burn center admission of $\geq 1$ day between 08/2011 and 09/2012 were invited to complete a questionnaire on long-term consequences of burns. We extended this sample with patients with severe burns ( $>20 \%$ total body surface area (TBSA) burned or TBSA full thickness $>5 \%$ ) treated between $01 / 2010$ and $03 / 2013$. Self-reported scar quality was assessed with the 
Patient Scale of the Patient and Observer Scar Assessment Scale (POSAS). Patients completed this scale for their -in their opinion- most severe scar $\geq 5$ years after burns.

Results: Our study included 251 patients with a mean\%TBSA burned of $10 \%$. The vast majority $(91.4 \%)$ reported at least minor differences with normal skin (POSAS item score $\geq 2$ ) on one or more scar characteristics and $78.9 \%$ of the patients' overall opinion was that their scar deviated from normal skin. Patients with severe burns had higher POSAS scores, representing worse scar quality, than patients with mild/intermediate burns, except for color, which was high in both groups. A longer hospital stay predicted reduced scar quality in multivariate analyses. In addition, female gender was also associated with a poorer overall opinion of the scar

Conclusion: This study provides new insights in long-term scar quality. Scars differed from normal skin in a large part of the burn population more than five years after burns, especially in those with severe burns. Female gender was associated with a poorer patients' overall opinion of their scar, which may be an indication of gender differences in perception of scar quality after burns.

Picture $\quad 1: \quad$ https://www.eventure-online.com/parthenuploads/89/9EBA/add_1_540908_7747eadc-e796-45cc-a6fe-6ddf96745da5.jpg

O9.07 The Matching Assessment using Photographs with Scars (MAPS) App: Reliability testing

\section{T Klotz, R Kurmis}

Royal Adelaide Hospital, ADELAIDE, Australia

Objectives: The Matching Assessment using Photographs with Scars (MAPS) is a scar assessment tool that was developed in 2005. MAPS has been recommended as a preferred scar assessment tools in a recent systematic review as it enables accurate relocation and reassessment of the scar. It is used across Australia, however, its distribution has been hampered by the need for the A4 paper manual.

In addition to the MAPS, the Modified Vancouver Scar Scale (mVSS) and a Patient Reported Outcomes questionnaire was digitised and incorporated into a new App to produce a comprehensive scar assessment package: ClinMAPS ${ }^{\text {тм}}$ Pro. At the time of development no other scar assessment Apps were available, making this the first of its kind.

To ensure the digitised MAPS is a reliable scar assessment, a reliability study was required.

Methods: The digitised MAPS within ClinMAPSTMPro was used for intra- and inter-rater reliability testing. Convenience sampling was utilised to recruit burns patients representing 42 scars, based on pre-determined power calculations. Three therapists, one experienced and two novice, acted as the assessors. Each therapist assessed pre-selected scars with the digitised MAPS. Reassessment of the same scar sites occurred 3-7 days later.

Results: Inter-rater reliability testing scores of the new electronic MAPS within the ClinMAPS ${ }^{\mathrm{TM}}$ Pro App showed fair to moderate agreement (combined Fleiss Kappa $=0.38-0.49$, $\mathrm{P}<0.0001)$. Intra-rater reliability scores between initial and repeat measures showed moderate to almost perfect agreement (Cohen's Kappa $=0.43-0.90, \mathrm{P}<0.0001$ ).

Conclusion: The digitisation of the previously paper based scar assessment manual ensures that

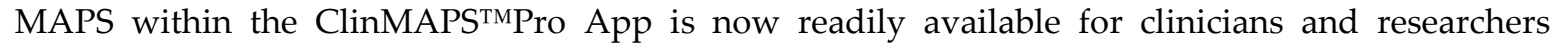
internationally. Results are integrated into electronic records or printed for paper records due to the report generation function. Results of the reliability testing of the MAPS component confirm its intraand inter-rater reliability.

O10.01 Laser speckle contrast imaging in children with scalds: Its influence on timing of intervention, duration of healing and care, and costs

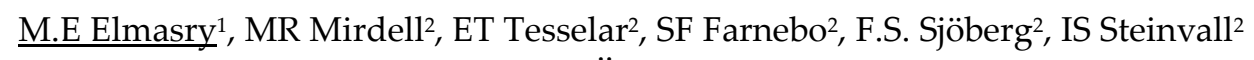

${ }^{1}$ Linköping university hospital, LINKÖPING, Sweden

${ }^{2}$ Linöping university hospital, LINKÖPING, Sweden

Objectives: Scalds are the most common type of burn injury in children, and the initial evaluation of burn depth is a problem. Early identification of deep dermal areas that need excision and grafting would save unnecessary visits and stays in hospital. Laser speckle contrast imaging (LSCI) shows promise for the evaluation of this type of burn. The aim of this study was to find out whether 
perfusion measured with LSCI has an influence on the decision for operation, duration of healing and care period, and costs, in children with scalds.

Methods: We studied a group of children with scalds whose wounds were evaluated with LSCI on day 3-4 after injury during the period 2012-2015. Regression (adjustment for percentage total body surface area burned (TBSA\%), age, and sex) was used to analyse the significance of associations between degree of perfusion and clinical outcome.

Results: We studied 33 children with a mean TBSA\% of 6.0 (95\% CI 4.4-7.7)\%. Lower perfusion values were associated with operation (area under the receiver-operating characteristic curve 0.86 , 95\% CI $0.73-1.00$ ). The perfusion cut-off with 100\% specificity for not undergoing an operation was $\geq 191$ PU units (66.7\% sensitivity and $72.7 \%$ accurately classified). Multivariable analyses showed that perfusion was independently associated with duration of healing and care period.

Conclusion: Lower perfusion values, as measured with LSCI, are associated with longer healing time and longer care period. By earlier identification of burns that will be operated, perfusion measurements may further decrease the duration of care of burns in children with scalds.

O10.02 Experience with a New Bedside Electrospun Nanofibrous Transient Skin Layer in Second Degree Burns

LHaik $^{1}$, Y. Ulman², E. Gur ${ }^{3}$, M. Haratz ${ }^{1}$, D. Kruchevsky², S. Zissman³ , H. Moser ${ }^{1}$, Y. Namir ${ }^{3}$

${ }^{1}$ The Sheba Medical Center, RAMAT GAN, Israel

${ }^{2}$ Rambam Health Care Campus, HAIFA, Israel

${ }^{3}$ Tel Aviv Sourasky Medical Center, TEL AVIV, Israel

Objectives: WHO reports more than $11 \mathrm{M} / \mathrm{y}$ people worldwide are burned severely enough to require medical treatment. The majority of burns are $2^{\text {nd }}$ degree burns requiring a complex healing process. This study evaluates a new system for burn treatment that produces a transient nanofibrous skin layer using electrospinning technology. This layer mimics the structure of the extracellular body tissue providing excellent medium for tissue integration and regeneration.

Methods: Patients with up to $10 \%$ TBSA burns were treated in 3 medical centers. The transient nanofibrous skin-layer was applied in-situ using the new system* activated from a distance, reducing pain and contamination risk. The nanofibrous layer is tailored to the shape and morphology of the wound. Opaque upon application, becoming transparent, allowing evaluation of wound bed without dressing removal. The layer remains on the wound until full epithelialization and self-peeling occur; a dry secondary dressing was used according to need and only in the first exuding days. Patients were allowed to shower regularly after 24-48 hours.

Results: Twenty-three (23) patients were recruited and treated. Mean age of 43Y (18-67), with $2^{\text {nd }}$ degree scald (20) and blast (3) burns of which up to 5\% TBSA were treated with the new system. Complete healing was achieved in $45 \%$ of patients on day $7^{\text {th }}$, in $90 \%$ of patients on day $14^{\text {th }}$. Pain was reduced immediately after dressing to 1.3 (VAS scale). No infections and no device-related adverse events were reported.

Conclusion: Large, hard to dress burns are easily treated with the new system, offering a minimally painful option for treatment and an effective healing process. The no-touch transient skin layer prevents potential infections and reduces dressing associated pain. It has excellent adherence even in challenging contours and was left on the wound until full recovery by day $14^{\text {th }}$.

* SpinCare ${ }^{\mathrm{TM}}$ Portable Wound Care System by Nanomedic Technologies Ltd.

O10.03 Regenerative Burn Surgery - evolution of an innovative concept with enzymatic debridement and in situ autologous cell and platelet-rich fibrin therapy

$\underline{\text { M. Waldner }}{ }^{1}$, T. Ismail ${ }^{2}$, H.J. Klein ${ }^{1}$, R. Schweizer ${ }^{1}$, A. Oramary ${ }^{1}$, T. Breckwoldt ${ }^{1}$, P. Giovanoli ${ }^{1}$, J.A. Plock ${ }^{1}$

1University Hospital Zurich, ZURICH, Switzerland

${ }^{2}$ University Hospital Basel, BASEL, Switzerland

Objectives: Deep partial thickness burns are traditionally treated by surgical necrectomy and split thickness skin graft coverage. The depth of surgical excision depends on the clinical experience 
of the surgeon to create a homogenous and plain wound-bed suitable for skin grafting. Split thickness skin grafts create donor site morbidity and increase the wound area in burn patients. Herein we present a novel concept including selective enzymatic debridement and coverage with autologous keratinocytes in combination with plated-rich fibrin (PRF) or fibrin glue, in deep partial thickness burns to reduce donor site morbidity and attenuate wound healing.

Methods: In a retrospective chart review, 22 patients with deep partial thickness burns treated with enzymatic debridement and autologous keratinocytes between 2017 and 2018 were included. Follow-up was between 4 months and 1 year.

Results: Patients mean age was $45 \pm 21$ yrs, male/female ratio was $16 / 6$, burn injuries affected 19.4 $\pm 15.25 \%$ TBSA, mean ABSI was 5.81(SD \pm 2.53$) .63 .6 \%(n=14)$ were treated with PRF, while $36.4 \%$ $(n=8)$ were treated with fibrin glue. The mean area treated was $7.56 \%$ TBSA, the mean time to full epithelialization was 29.9 days ( $\mathrm{SD} \pm 19.6$ ) , the mean hospitalization time was 30 days ( $\mathrm{SD} \pm 26.8$ ). $31.8 \%$ $(n=7)$ of patients needed additional split thickness skin grafting, $13.6 \%$ of patients had postoperative wound infections.

Conclusion: Enzymatic debridement in combination with autologous keratinocytes and $\mathrm{PRF} /$ fibrin glue represents a powerful, novel regenerative concept allowing for selective wound debridement and minimal donor site morbidity for in situ skin engineering. To avoid the need for secondary skin grafting, further studies to optimize indications according to anatomical regions and timing between debridement and coverage need to be performed. Also, optimized cell isolation protocols and technology might facilitate the procedure.

O10.04 Clinical study of nanofibrillar cellulose dressing for skin graft donor site treatment

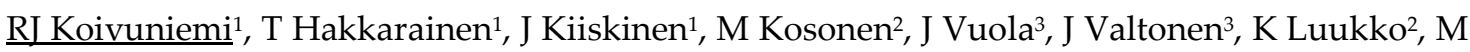
Yliperttula ${ }^{1}, \mathrm{H} \mathrm{Kavola}^{3}$

${ }^{1}$ University of Helsinki, HELSINKI, Finland

${ }^{2}$ UPM-Kymmene Corporation, HELSINKI, Finland

${ }^{3}$ Helsinki University Hospital and University of Helsinki, HELSINKI, Finland

Objectives: Management of skin graft donor sites is a concern due to pain and discomfort, and due to delayed wound healing, particularly in elderly patients or in patients with poor wound healing competence. A dressing that would provide optimal healing, low costs and minimal pain with no or few dressing changes would be a preferred choice for treatment of donor sites. The aim of this prospective, single center clinical trial was to evaluate the efficiency of a nanofibrillar cellulose wound dressing (FibDex® by UPM-Kymmene Corporation) in treatment of donor sites.

Methods: FibDex ${ }^{\circledR}$ was intra-individually compared to a commercial polylactide-based dressing, Suprathel ${ }^{\circledR}$. Twenty-four patients requiring skin grafting with mean age of $49 \pm 18$, were enrolled in the study performed at Helsinki Burn Centre. Primary outcome measure was wound healing time, assessed by self-detachment of the dressings. Secondary outcomes, including the epithelialization percentage, subjective pain, the scar appearance assessed using the Patient and Observer Scar Assessment Scale (POSAS), skin elasticity and transepidermal water loss (TEWL), were evaluated at one and six months post-operatively.

Results: No statistically significant differences were observed between FibDex ${ }^{\circledR}$ and Suprathel ${ }^{\circledR}$ regarding wound healing time, epithelialization, experience of pain, or TEWL. The mean healing time for both dressings was 18.5 days $\left( \pm 5.3\right.$ for FibDex ${ }^{\circledR}, N=24 ; \pm 4.6$ for Suprathel $\left.{ }^{\circledR}, N=16\right)$. Significant differences were observed in the POSAS results for thickness ( 1 month; $\mathrm{P}=0.04)$ and vascularity (6 months; $\mathrm{P}=0.008)$ in the Observer score, in favour of FibDex ${ }^{\circledR}$ over Suprathel ${ }^{\circledR}$. Morever, skin elasticity was significantly improved with FibDex ${ }^{\circledR}$ in terms of viscoelasticity $(P=0.016)$ and elastic modulus $(\mathrm{P}=0.01)$ at one month post-operatively.

Conclusion: FibDex® is an efficient dressing for the treatment of skin graft donor sites, and its performance was shown to be comparable to that of Suprathel ${ }^{\circledR}$ in terms of wound healing time, epithelialization and pain. However, the elasticity of the scar is significantly improved with FibDex®. 
O10.05 Prototype of an airlock without doors: easing daily care and preventing external contamination

\author{
C. Flageul $^{1}$, M. Ferrand ${ }^{1}$, C. Beauchene ${ }^{1}$, M. Mimoun ${ }^{2}$ \\ ${ }^{1}$ CEREA, MARNE LA VALLÉE CEDEX 2, France \\ ${ }^{2}$ Hôpital Saint-Louis, PARIS, France
}

Objectives: Treatment of patients suffering from severe burns requires a controlled atmosphere in order to limit as much as possible any external contamination. A room with a pressurized and ventilated airlock can fulfill this role. We present a new prototype of an open ventilated section which effectively prevents such external contamination. Removing doors should ease the medical workers daily care.

Methods: Absence of external contamination is studied with Computational Fluid Dynamics (CFD). The room is a digital twin of the one existing in the Saint-Louis hospital located in Paris and dedicated to light to medium burns. Sensitivity to both the mesh refinement and the turbulence models are performed with the open-source CFD platform Code_Saturne. The efficiency of the aeraulics concept is tested with an Eulerian-Lagrangian approach. Lagrangian particle's diameter range between 0.1 and 10 microns, which is representative of potentially harmful external contaminants.

Results: Our simulations show that the prototype is capable of reducing the amount of external contamination.

Conclusion: Our approach validates the prototype. Of course, marginal improvements are still possible, and can be investigated using additional simulations. The next step for the validation of the prototype is an experimental validation in realistic conditions.

\title{
O10.06 THE DEVELOPMENT OF AN ADULT, BURN SPECIFIC PATIENT CONCERNS INVENTORY (PCI)
}

\section{I.A.G Gibson ${ }^{1}$, S Spencer ${ }^{2}$, J Yarrow ${ }^{1}$, S.N Rogers², K Shokrollahi ${ }^{3}$}

${ }^{1}$ The Welsh Centre for Burns \& Plastic Surgery, BURNHAM-ON-CROUCH, United Kingdom

2Edge Hill University, ORMSKIRK, United Kingdom

${ }^{3}$ Mersey Burns Centre, LIVERPOOL, United Kingdom

Objectives: Burn injuries are a major source of physical and psychological trauma. The need for a holistic approach to burn care is essential if the many issues that a patient may encounter such as physical symptoms, psychological issues and reduced physical function are to be fully addressed. Identifying the issues and concerns that matter most to patients is a difficult process during an outpatient consultation. Consequently some concerns may not be discussed and opportunities to optimise patient-centred care may be missed. The Patient Concerns Inventory (PCI) is a prompt list that allows patients to self-report concerns prior to consultation. It has been successfully developed for a number of other medical specialities. The aim of this study was to develop a PCI for adult burns patients.

Methods: Content for the PCI was generated from three sources: Burns Health Related Quality of Life (HRQoL) tools, thematic analysis of one to one interviews with 12 adult burns patients and Multi-Disciplinary Team (MDT) members from two regional burns centres. Content was refined using a Delphi consensus technique, with patients and staff members, using SurveyMonkey.

Results: A total of 111 individual items were generated from the three sources. The Delphi process refined the total number of items to 58. The main emergent domains were physical and functional well-being (18 items), psychological, emotional and spiritual well-being ( 22 items), social care and social well-being ( 7 items) and treatment related concerns (11 items).

Conclusion: The Adult, Burns Specific Patient Concerns Inventory is a 58 item, Holistic Needs Assessment (HNA) Tool designed to be used in the outpatient clinic. The PCI has been carefully constructed to represent the most common concerns and issues experienced by adult burn patients. It offers a novel tool to improve doctor/patient communication in the outpatient setting and to assist in the identification of patient concerns. 
O11.01 Blood glucose level and outcome in septic burn patients

A. Lavrentieva, E. Sourla, C. Giannaki, V. Renesis, C. Theocharidou, M. Bitzani

Papanikolaou Hospital, THESSALONIKI, Greece

Objectives: The goal of this study was to analyze the relationship between blood glucose level and outcome and to evaluate the effect of the route of nutrition (EN versus PN) on glucose levels.

Methods: Forty-four burn patients with septic complications were included in the study (mean TBSA burned $40 \pm 14 \%$, mean SOFA on admission 3.8 \pm 2.2 ). A moderate glycemic control was applied in all patients. Standard deviation of blood glucose levels per patient was used as glucose variability indicator. The median glycemia and the largest change over the first $72 \mathrm{hrs}$ were included in a multivariate analysis that was performed to determine the association of glucose level with outcome.

Results: Of the 44 patients, 27 (61.3) had a favorable outcome. Median BG level was $8.7 \mathrm{mmol} / \mathrm{L}$ (IQR 5.6-10) in patients with a favorable outcome compared to $10.0 \mathrm{mmol} / \mathrm{L}$ (IQR 7.2-11.7) for patients with an unfavorable outcome $(\mathrm{p}<0.01)$. Median BG level variability was 1.6 vs. $2.2 \mathrm{mmol} / \mathrm{L}$ in patients with and without a favorable outcome, respectively $(\mathrm{p}<0.01)$. In multivariate analysis, an increased median BG level over the first $72 \mathrm{~h}$ of sepsis was found to be an independent predictor of poor prognosis [OR $=0.41 ; 95 \% \mathrm{CI}(0.23-0.7), \mathrm{p}=0.006]$. Parenteral nutrition was used in $21 \%$ of patients with unfavorable outcome and in $19 \%$ of patients with favorable outcome. We did not find the association of peak glucose levels and glucose variability in patients with enteral and parenteral routes of feeding.

Conclusion: This study demonstrated that both high glucose levels and high glucose variability is associated with increased mortality in septic burn patients. The use of parenteral nutrition as compared to enteral nutrition had no effect on glucose levels and glucose variability.

O11.02 Bisphophonates as inhibitors of carbapenemases.

\section{A. Pleshkov, T. Voroshilova, S. Shapovalov, A. Panov}

Nikiforov`s Russian Center of Emergency and Radiation Medicine, SAINT-PETERSBURG, Russia

Objectives: To assess the clinical response among patients with burn sepsis caused by MBLsproducing Gram-negative bacilli treated with combination including bisphosphonates (clodronic acid) and carbapenems.

Methods: Our previous in vitro studies showed high synergistic effect of bisphosphonates, known for its chelating properties, and carbapenems against metallo- $\beta$-lactamases (MBLs) producers. Retrospective cohort study included 19 patients with burn sepsis treated in ICU. The infectious process was caused by carbapenemase-producing K. pneumoniae, P. aeruginosa and/or A. baumannii. Carbapenemase detection was performed by screening tests (E-test) verified with molecular methods (PCR). 14 patients received combination of clodrone acid ('Bonefos', Bayer Oy) and carbapenem ('Meropenem', Astra Zeneca), whereas 5 patients were administered carbapenems in high doses (Meropenem, 2g/8h). Clinical indicators, such as WBC count, ESR, CRP, PCT, Creatinine, Ca++ and microbiological colonies counting (CFU) were observed.

Results: VIM-producing P. aeruginosa, NDM-producing K. pneumoniae, OXA-23 and OXA-40producing A. baumannii were identified in microbiological samples. After 7 to 10 days of treatment in combination group we obtain significant clinical response in all cases stated in statistically significant $(\mathrm{p}<0.05)$ improvement of clinical and microbiological indicators: WBC count, ESR, PCT, CFU $(\mathrm{p}=0.001)$. Monotherapy group did not show statistically significant changes along with progression of sepsis symptoms. There were no adverse reactions and side effects of prolonged intravenous administration of clodronic acid.

Conclusion: We suggest that bisphosphonates may be effective inhibitors of MBLs due to its chelating properties. There are several commercially available bisphosphonates approved for clinical practice in many countries. Clodronic acid seemed most suitable for this purpose.

O11.03 Parenteral Iron treatment for postoperative anemia in burns 
J Carbajal-Guerrero, P Gacto-Sanchez, M Mendoza-Prieto

Hospital Virgen del Rocio, SEVILLA, Spain

Objectives: In burned patients $50 \%$ of transfusions are not perioperative, with etiology attributed to the anemia of the critical patient. $17 \%$ of transfusions in those patients occur after their last surgery postoperative period during the acute phase. Transfusions is one of the predictive factors in reducing burns survival rates. An infection risk of $13 \%$ has been estimated for each transfused red blood cell concentrate, with non-perioperative transfusions having the greatest influence on survival, thus suggesting a greater emphasis on minimizing this procedure.

The main objetive of our study is to clarify whether intravenous iron supplementation in patients with iron deficiency reduced the need for transfusions after the surgical treatment phase.

Methods: Burned patients showing progressive anemization after concluding the surgical phase, and subjected to at least two surgeries, were selected during eleven months. 23 patients were evaluated to detect iron deficit and treated with parenteral iron if the diagnostic was confirmed.

Subsequently, these patients were compared with a group of historical controls, to clarify whether parenteral iron administration reduced the need for blood transfusions after the surgical phase.

Results: Number of concentrates transfused in the postsurgical phase (from $48 \mathrm{~h}$ after the last surgery) were analyzed. One out of every four patients in the control group $(25 \%)$ were transfused at least two red blood cell concentrates whereas no transfusions were needed after parenteral iron administration in the case group $(\mathrm{p}=0.03)$.

When changing transfusions into a dichotomous variable, $44 \%$ of the patients in the control group received transfusions 48 hours after their last surgery $(p=0.03)$, compared to $0 \%$ in the case group.

Conclusion: Iron treatment of iron deficiency seems to reduce transfusion necessities once the surgical phase was completed. Hence, in burned patients showing progressive anemia, iron deficiency should be assessed and parenteral supplementation must be administered if required.

O11.04 Efficacy of esmolol during early postresuscitation period in burn ICU patients.

A. Lavrentieva, C. Giannaki, E. Sourla, V. Renesis, M. Stamatopoulou, A. Kolesnikova, M. Bitzani

Papanikolaou Hospital, THESSALONIKI, Greece

Objectives: To evaluate the efficacy of esmolol infusion during the early post resuscitation phase in ICU burn patients.

Methods: From April 2015 to February 2018, we enrolled 22 patients admitted to burn intensive care unit who were receiving vasopressor infusion and had tachycardia despite adequate volume expansion. All patients received a continuous intravenous infusion of esmolol, targeted to heart rate $80-90 / \mathrm{min}$, while receiving vasopressors. The resuscitation of burn patients was based on the unit's resuscitation protocol, crystalloid driven resuscitation was used. Hemodynamic parameters, obtained by arterial pulse contour analysis, were evaluated before and $6 \mathrm{hrs}$ after esmolol infusion.

Results: Median peak esmolol infusion was $50(25-50) \mu \mathrm{g} / \mathrm{kg} / \mathrm{min}$. Median norepinephrine infusion rate at the initiation of esmolol infusion was $0.60(0.14-0.83) \mu \mathrm{g} / \mathrm{kg} / \mathrm{min}$. Median peak norepinephrine infusion rate during esmolol infusion was $0.66(0.18-0.95) \mu \mathrm{g} / \mathrm{kg} / \mathrm{min}$. Mean arterial pressure at initiation of esmolol infusion was $77 \pm 6 \mathrm{mmHg}$, with mean cardiac index of $3.2( \pm 1.5)$ $\mathrm{L} / \mathrm{min} / \mathrm{m}^{2}$ and stroke volume index of $35( \pm 9) \mathrm{mL} / \mathrm{m}^{2}$. After esmolol infusion blood pressure was unaltered $(75 \pm 10 \mathrm{mmHg})$, cardiac index was decreased whereas stroke volume was increased compared with before esmolol therapy (cardiac index $2.9 \pm 1 \mathrm{~L} / \mathrm{min} / \mathrm{m}^{2}$, stroke volume index $42 \pm 11$ $\left.\mathrm{mL} / \mathrm{m}^{2}, \mathrm{p}<0.05\right)$. Twenty patients $(91 \%)$ patients achieved target heart rate within $6 \mathrm{hrs}$ of esmolol infusion. Mean overall duration of esmolol infusion was $64( \pm 37)$ hours. Tissue perfusion, estimated by lactate levels $(2.7 \pm 1.5$ vs. $2.2 \pm 0.9 \mathrm{mmol} / \mathrm{L}, \mathrm{p}=0.055)$ was also slightly improved after esmolol therapy. 
Conclusion: Esmolol infusion in the early postresuscitation period was associated with an increase in stroke volume index, small decrease in cardiac index and improved tissue perfusion assessed by serum lactate levels.

O11.05 Heterogeneity in Burns Intensive Care Management. A Retrospective Review of Patients Admitted over two years to a Quaternary Burns Intensive Care Unit.

IF Mewton, J Gowardman, J Miller

Royal Brisbane and Women's Hospital, HERSTON, BRISBANE, Australia

Objectives: To review management of intensive care patients with burns. Considering resuscitation received, sedation and analgesic regimens on admission in theatre and at discharge. The role of routine screening for multi-resistant organisms and the microbiological management of burns patients in intensive care.

Methods: With ethical approval a retrospective chart review of patients admitted over two years to an adult Quaternary Burns Unit with a primary diagnosis of a Burn.

Results: Ninety-three patients, sixty-four males $(70 \%)$ and twenty-seven $(30 \%)$ females aged between seventeen and eighty-five years were admitted in 2016/2017. Seventeen (18.3\%) died in intensive care with mortality associated with age $>80$ years, female gender, a TBSA burn $>80 \%$, a calculated Parkland resuscitation greater than twenty litres, hypothermia and acidaemia.

Intensive Care documentation of initial fluid resuscitation was complex and poorly recorded. Sixty-eight patients exceeded their Parkland calculation despite under documentation of early fluid administration.

Eighty-nine patients were sedated on admission with fourteen different analgesic/sedative regimens varying from one to five agents. There was significant intraoperative analgesic dosing, with patients receiving a mean $0.2 \mathrm{mg}$ morphine/minute for the first operation versus $1 \mathrm{mg} / \mathrm{min}$ for the second. Discharge analgesic regimens were multiple utilising opiates, antipsychotics and neuropathic agents. Males were more likely to receive neuropathic agents, previous substance users higher opiate doses and non-substance users more antipsychotic prescribing.

Multiple Resistant Organism (MRO) screening was performed with a median frequency of 2.7 days at a cost of $\$ 25,000$ AUD with new organisms identified in $2.5 \%$ of screens. Microbiologically patients had multiple organisms in multiple sites. Three of the top four organisms were MROs. Fiftythree patients were discharged without antibiotics.

Conclusion: The unit demonstrates significant heterogeneity in the Intensive Care Management of severe burn trauma. Improving quality of care could include standardising analgesic/sedation regimens and reducing or removing screening for MROs.

\section{O11.06 ANALYSIS OF EARLY IMMUNOLOGICAL CHANGES IN A MAJOR BURNED PATIENTS COHORT}

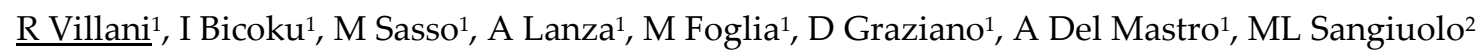

${ }^{1}$ A. Cardarelli Hospital, NAPLES, Italy

${ }^{2}$ A Cardarelli Hospital, NAPLES, Italy

Objectives: Our aim is to characterize the still mostly unknown early immunological changes in major burned patients in order to study correlations with mortality and optimize the current therapies.

Methods: We studied 8 patients admitted in the Burns Unit of Cardarelli Hospital (Naples) for major burns from November 2018 to January 2019. Venous blood withdrawal was performed at acceptance (T0) and after 10 days (T10) to analyze humoral and cell-mediated immunity (white blood cells count, complement, total IgA, IgM and IgG levels, IgG subclasses, lymphocyte subpopulations). For each patient demographic data, total Burned Skin Area (TBSA), vital parameters, renal and hepatic analytes, sepsis onset, survival were recorded. Means and frequencies were calculated.

Results: Mean age was 57 years (range 13-80 years), all Caucasian, 2 women; mean TBSA was $33.3 \%$ (13.5 to 100). All the patients showed neutrophilia, monocyte increase, varied renal and hepatic impairment. One patient (male, 75 yo, TBSA 100\%) died at T0 after acceptance. He had low CD4 ${ }^{+}$cells 
and IgG. One patient (male, 82 yo, TBSA 36\%) died at T10: he had low CD4 ${ }^{+}$and CD8 ${ }^{+}$cells, low IgM and IgG with IgG1-2 deficiency at T0, with worsening at T10. The other 6 patients survived: interestingly, 5 showed low $\mathrm{CD}^{+}$and IgG subclasses deficit (4 had low IgG1-2-3, one low IgG1). One survivor (male, 30 yo, TBSA 49.5\%) became septic at T10, showing low CD4+ and IgG with low IgG12-3. No patient had IgG4 deficit.

Conclusion: We are confident that by increasing the number of patients and the time of analysis we can confirm the significance of our observations and plan new therapeutical interventions aimed at reverting the immunologic impairment.

O12.01 Biological dressings as an aid to expedite wound healing in graft donor sites

$\underline{\text { S Rahman }}{ }^{1}$, B Langridge ${ }^{2}$, A Al-Hadad ${ }^{3}$, R Ali Khan ${ }^{4}$ M Junejo ${ }^{3}$, A Mosahebi ${ }^{3}$

${ }^{1}$ Bradford Royal Infirmary, BRADFORD, United Kingdom

2Department of Medicine, UCL, London, LONDON, United Kingdom

${ }^{3}$ Division of Surgery \& Interventional Science, LONDON, United Kingdom

${ }^{4}$ University College Cork, CORK, Ireland

Objectives: Currently there is no definitive dressing type regarding the management of split thickness skin graft(STSG) donor sites which can be a subject of pain, exudation, infection as well as delayed healing. The literature reports both biological and non-biological as the two main types, however, there is no conclusive evidence regarding the ideal one.

The objective of this study was to perform a systematic review and meta-analysis of relevant studies and compare both groups by assessing outcomes in their application to split skin graft donor sites.

Methods: The Preferred Reporting Items for Systematic Reviews and Meta-Analyses (PRISMA) statement standards was used to conduct this study. Electronic databases including MEDLINE, EMBASE, CINAHL and the Cochrane Central Register of Controlled Trials (CENTRAL) were searched by two authors (SR and BL). Data analysis was performed with Review manager 5.3.

Results: Seven studies in total consisting of five RCTs and 2 observational studies were identified. Wound healing time was faster in biological dressings compared to the non-biological group (odds ratio, $0.14 ; 95 \% \mathrm{CI}, 0.05-0.44$ ). A higher epithelialisation rate was also noted for biological dressings. There was no difference in the infection rate between the two study groups (odds ratio $0.31 ; 95 \% \mathrm{CI}, 0.09-1.04$ ) or wound exudation (odds ratio $0.31 ; 95 \% \mathrm{CI}, 0.01-8.28$ ). The pain level experienced during dressing changes in both groups was reported to be similar

Conclusion: The rate of epithelialisation and wound healing is greater for STSG donor sites when treated with biological dressings but they offer no advantage in terms of reducing pain, infection or exudation.

O12.02 First experiences in the treatment of large superficial burns up to 49\%TBSA with pure silk

ISchiefer, PC Fuchs

Clinic for Plastic Szurgery, Hand Surgery, Burn Care Center, COLOGNE, Germany

Objectives: During the treatment of superficial burn injuries patients are often confronted with painful dressing changes. Therefor we often treat these wounds with very expensive materials like nylon mesh combined with animal collagen that can stay on the wound and slowly peel off during re-epithelialization.

Due to our good experiences with the treatment of small burn injuries with pure natural silk as cost-efficient material, we now also started treating large burn injuries for the first time with silk.

Methods: Altogether 26 patients with superficial burn injuries over $10 \%(10.5-49 \%)$ of the body surface area were treated with pure silk. Afterwards the wound healing, number of operations and the patient's satisfaction were evaluated.

Results: In 24 of 26 cases all wounds healed unproblematically without dressing change, infection or further operations. In three cases, mostly chemical burns, initial burn depth assessment was not correct and skin grafting became necessary during wound healing. All patients were satisfied with the treatment, though stated a feeling of stiffness in dressings covering joints. 
Conclusion: Altogether silk is an interesting, cost-efficient material for the treatment of superficial burns. During the treatment of larger areas a careful wound depth evaluation is very important to avoid infections and further operations during the wound healing.

O12.03 The nylon-reinforced silver sodium carboxymethylcellulose dressing reduces the infection risk and shortens hospital stay in treatment of deep dermal burns

MA Argirova ${ }^{1}$, YZ Zayakova $^{2}$, TD Dimitrova ${ }^{1}$

${ }^{1}$ UMHATEM „N.I. Pirogov,", SOFIA, Bulgaria

${ }^{2}$ Department of burns and plastic surgery, VARNA, Bulgaria

Objectives: The local treatment of the burn wounds is an important stage of the complex treatment of this type of trauma. The key considerations in choosing of dressing are the level of its antiseptic effect, its bioactive effect on epithelialisation and ability for controlling the wound infection. The aim of the study is to determine the efficiency of nylon-reinforced silver sodium carboxymethylcellulose dressing in comparison with the local conventional therapy.

Methods: A prospective clinical study was conducted between March and December 2018 in Burn Department. A total of 52 patients having acute burn wounds with various areas, depth and localization were studied. Demographic and statistical analyses were carried out. The clinical efficiency of the dressing was validated by monitoring the following parameters: antibacterial effect, wound time to healing, daily pain scores, number of dressing changes, patient comfort, dressing adherence, nursing time and length of hospital stay.

Results: Most of the burns were of type IIB (53\%), type IIA (23\%) and type III (23\%). The mean period of epithelisation time was 11.61 days. The mean hospital stay of the treated patients was 7.88 days. The results showed average pain score of 2.24. Patients treated with this dressing have lower number of wound dressing change (2.25) and nursing time in comparison with the conventional dressings used in the department. No side effects were observed. The obtained results were statistically validated. The wounds without bacterial growth in the beginning remained sterile until healing. Bacterial growth was significantly reduced during the treatment and bacterial contamination was not isolated in the end of the treatment. Patient comfort was measured through patient questionnaire classified into three categories.

Conclusion: The ease of use, non-adhesion, high absorbency, powerful antibacterial effect, lower frequency andpainless dressing change are the key advantages of the silver sodium carboxymethylcellulose dressings over the older silver agents.

O12.04 Epicite-hydro, first dressing to the wound bed preparation in full thickness burns

P Rodriguez-Ferreyra, J Bernal-Casas

Instituto de Salud del Estado de Mexico, TOLUCA, Mexico

Objectives: Full-thickness burns are a challenge that will have a direct impact on the quality of life of the patient, cost benefit for the hospital and a surgical goal; Dermal regenerators and cadaveric skin are the best options; However, in our country, the availability of one of both is practically null. Accidentally we found that one of the benefits of Epicitehidro (non-woven 3D network biopolymer of pure Nano-cellulose fibers) was to maintain a humid environment That is why we present the experience in a Children Burn Unit with Epicitehydro, in patients with full thickness burns, used in the wound bed preparation after skin resection.

Methods: Patients with a full thickness areas, who were made fascio-cutaneous or full cutaneous scarectomy (It was performed in the first 48 hours after the burn with clinically determining that the burn was of full thickness) and applied the Epicitehydro, (second dressing was bactigras, changed every 2 days); after 7 days removed the Epicite hydro and evaluate if the characteristics of the wound allowed to place autografts.

Results: 17 patients, age between 7 and 17 years. There were 11 boys and 6 girls. TBSA involvement varied between 20 to $35 \%$, with full thickness areas between 5 to $10 \%$; etiology was fire in all the patients. There were no infections, Pain was evaluated with VAS: 1-3 points; had good 
adherence; no side effects, no changes of Epicitehydro were necessary and after 7 days the wound was ready to undergo to graft.

Conclusion: Someone Hospitals in Low-income countries have a big problem with the immediate availability of cadaver skin or dermal regenerator for full thickness burns. That's why the use of this new Nano-cellulose dressing that promotes the wound bed preparation has been an excellent option. We are working now in a prospective and comparative study to conclude the real benefit of this dressing.

Picture

$1:$

https://www.eventure-online.com/parthenuploads/89/9EBA/add_1_539296_dbce6042-170b-4cbe-9387-ef601e32ffa6.jpg

Caption 1: evolution with the use of epicite in right lateral trunk

O12.05 Experienced with Nanocellulose- Epicite-hydro- for superficial and deep partial thickness burns

P Rodriguez-Ferreyra, J Bernal-Casas

Instituto de Salud del Estado de Mexico, TOLUCA, Mexico

Objectives: In low-income countries, it is difficult to have equipment to establish the diagnosis of partial thickness burns, since there is no equipment such as laser Doppler. However isnecessary cover the woud with a dressing to prevent the wound drying and perhaps starting the treatment of the burn. We choose Epicitehydro, a non-woven 3D network biopolymer of pure Nano-cellulose fibers, provides protection against pathogens, prevents cell adhesion with the wound, biocompatible, liquid absorption and retention capacity, moisture content control and avoid pain.

Objective: Present the experience in a Children Burn Unit with Epicitehydroas a first-line dressing in patients with superficial and deep partial thickness or indeterminate burns by scald, who arrive during the first 48 hours after the burn, to whom Epicitehydrowas applied.

Methods: Patients with scalds, whom arrive in first 48 hours, accepted the use of Epicitehydro, after cleaning the burn was applied Epicitehydro.

Results: 50 patients, age between 1 and 35 years. There were 29 males and 21 female. TBSA range between 2 to $54 \%$, the superficial partial thickness was the most often. Time average from the burn to Epicitehydro application was 26 hours. All the patients presented a satisfactory evolution; so we waited until 5 th or 7 th days to proceed to remove the dressing and we observe: epithelialization in $100 \%$ if TBSA was less than 5\%; epithelialization in 95\% if TBSA less than $10 \%$ and the patients with more than TBSA $>11 \%$ had epithelialization between 80 to $90 \%$, and someone required autograft or allograft to obtain a complete epithelialization. Also, we observed decreased in the inflammatory response and pain.

Conclusion: The use of this new dressing promotes fast and excellent epithelialization in superficial and deep partial thickness injury, especially scald burns. In addition, we found, no pain during use and removal of the dressing, and there were no infections.

Picture $\quad 1: \quad$ https://www.eventure-online.com/parthenuploads/89/9EBA/add_1_539299_275a73ff-c54e-46af-a5cf-b16a7d957b80.jpg

Caption 1: Evolution with Epicite from day 1- day 7 to 1 month

O12.06 Hyaluronic acid-based wound dressings in the treatment of pediatric facial burns - an improved standard of care?

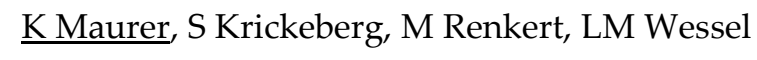

University Mannheim Medical Centre, MANNHEIM, Germany

Objectives: $25 \%$ of thermal injuries in infants and toddlers include the face and neck region. Wound dressings for these patients should be easily applicable, reduce pain and pruritus, work as an occlusive dressing to prevent wound infection, enable full mobility and avoid painful dressing changes. Established wound dressings consisting of polylactide, cellulose, hydrocolloid or nonadhesive gauze require protective secondary dressings which are difficult to apply on children's faces. Frequent dressing changes are necessary for wound assessment and hygiene reasons, requiring prolonged hospitalisation. With that in mind, we share our experience using a hyaluronic acid-based 
adhesive membrane (Hyalosafe $\left.{ }^{\circledR}\right)$ as the standardized treatment of pediatric facial burns at the University Medical Centre Mannheim pediatric burn centre.

Methods: From 6/2016 to 12/2018, we treated 72 patients with mid-dermal burns to the face and neck with a gentle debridement followed by the application of Hyalosafe ${ }^{\circledR}$ without a secondary wound dressing. Patients with isolated facial burns remained in outpatient care or were hospitalised for no more than 2 days.

Results: Our experience with Hyalosafe ${ }^{\circledR}$ showed easy applicability, high acceptance by patients and hardly any restrictions to their daily routine. In infants and toddlers, the film dressing provided a temporary hygienic occlusive wound covering unaffected by contact with food or saliva. The transparency of the dressing allowed unrestricted wound assessment, while the shedding of Hyalosafe ${ }^{\circledR}$ after reepithelialization rendered further dressing changes unnecessary. We documented no cases of wound infection, prolonged pain or pruritus. Outpatient follow-up showed speedy recovery with scar-free reepithelialization in the majority of cases and some rare localized scarring.

Conclusion: The treatment of pediatric facial burns with Hyalosafe ${ }^{\circledR}$ as a temporary epithelial substitute proves to be an effective alternative regarding applicability, compliance, patient comfort, hospitalisation and wound healing.

O12.07 Microbial cellulose dressing compared with silver sulphadiazine for the treatment of partial thickness burns: A prospective, randomised, clinical trial

\section{M.E Elmasry}

Linköping university hospital, LINKÖPING, Sweden

Objectives: The current treatment for partial thickness burns at the trial site is silver sulphadiazine. Its deleterious effect on wound healing, together with the need for repeated, often painful, procedures, has brought about the search for a better treatment. Microbial cellulose has shown promising results that avoid these disadvantages. The aim of this study was therefore to compare microbial cellulose with silver sulphadiazine as a dressing for burns.

Methods: All patients who presented with partial thickness (superficial and deep dermal) burns from October 2014 to October 2016 were screened for this randomised clinical trial. Twenty patients were included in each group: the cellulose group was treated with microbial cellulose sheets and the control group with silver sulphadiazine cream $10 \mathrm{mg} / \mathrm{g}$. The wound was evaluated every third day. Pain was assessed using the Face, Legs, Activity, Cry, Consolability (FLACC) scale during and after each procedure. Other variables recorded were age, sex, percentage total body surface area burned (TBSA\%), clinical signs of infection, time for epithelialisation and hospital stay. Linear multivariable regression was used to analyse the significance of differences between the treatment groups by adjusting for the size and depth of the burn, and the patient's age.

Results: Median TBSA\% was 9\% (IQR 5.5-12.5). The median number of dressing changes was 1 (IQR 1-2) in the cellulose group, which was lower than that in the control group (median 9.5, IQR 616) $(\mathrm{p}<0.001)$. Multivariable regression analysis showed that the group treated with microbial cellulose spent 6.3 (95\% CI 0.2-12.5) fewer days in hospital ( $\mathrm{p}=0.04)$, had a mean score that was 3.4 (95\% CI 2.5-4.3) points lower during wound care ( $\mathrm{p}<0.001)$, and 2.2 (95\% CI 1.6-2.7) afterwards $(\mathrm{p}<0.001)$. Epithelialisation was quicker, but not significantly so.

Conclusion: These results suggest that the microbial cellulose dressing is a better first choice for treatment of partial thickness burns than silver sulphadiazine cream. Fewer dressings of the wound were done and, combined with the low pain scores, this is good for both the patients and the health care system. The differences in randomisation of the area of burns is, however, a concern that needs to be included in the interpretation of the results.

O13.01 Risk factors for burn accidents in children of 0-4 years old: a prospective study

E.E. Van Zoonen ${ }^{1}$, A. Pijpe ${ }^{2}$, M.E. Van Baar ${ }^{2}$, M.K. Nieuwenhuis ${ }^{2}$, C.H.M. Van Schie ${ }^{1}$, A. De Vries ${ }^{3}$ ${ }^{1}$ Dutch Burns Foundation, BEVERWIJK, Netherlands

${ }^{2}$ Association of Dutch Burn Centres, BEVERWIJK, Netherlands

${ }^{3}$ Red Cross Hospital, BEVERWIJK, Nederland 
Objectives: Children 0-4 years old are more frequently admitted in the Dutch Burn Centres than any other age group. For this reason the Dutch Burns Foundation has been organising prevention campaigns aimed at caregivers of young children. These campaigns are based on known risk factors. However, the most recent study on risk factors for burn accidents is more than 10 years old. The aim of this study therefore, was to determine current risk factors for burn accidents in children 0-4 years of age.

Methods: Eligible were children 0-4 years old treated in the in- or outpatient clinic of the three Dutch burn centres. Information on personal-, environmental- and behavioural circumstances during the burn accident was prospectively collected during one year in 2017-2018 from patient records and a structured questionnaire. Relevant determinants of behavioural- and environmental circumstances during the accident were identified. Personal characteristics were compared to the general population in order to identify risk factors for burn accidents.

Results: In total 533 patients were included (boys: 53\%). Their median age was 18 (12 SD) months. In $41 \%$ of the accidents the cause was tea $(68 \%)$, coffee $(20 \%)$ or hot water $(12 \%)$ spilled from a cup. In $55 \%$ of these cases the child was actively pulling the cup. Accidents with spilled hot liquid happened often in close proximity to the person who was responsible for the child; $89 \%$ was located in the same room and 58\% saw the accident happen. Patients with a migration background were at increased risk $(R R=1.83)$ for burn accidents as well as patients who lived in a neighbourhood with a low Social Economic Status (SES).

Conclusion: Eighteen months of age, a migration background and living in a low SES neighbourhood are important risk factors for burn accidents in children 0-4 years old. Prevention programs should focus on these risk factors.

\section{O13.02 The Global Burn Registry of the World Health Organization}

M Peck $^{1}$, H Falk ${ }^{2}$, D Meddings ${ }^{3}, \underline{\text { Y Singer }}^{4}$

${ }^{1}$ Arizona Burn Center, PHOENIX, USA

${ }^{2}$ Emory Rollins School of Public Health, ATLANTLA, USA

${ }^{3}$ World Health Organization, GENEVA, Switzerland

${ }^{4}$ Victorian Adult Burn Service, PRAHRAN, Australia

Objectives: WHO has launched the Global Burn Registry - the first ever global platform allowing for standardized data collection from burn survivors. This new resource will provide health facilities with a clear picture of the factors most likely to contribute to burns and the populations at greatest risk in their settings, with a view to prioritizing prevention programmes.

Methods: Progress in burn prevention in low- and middle-income countries is hindered because data collection for burn injuries is weak, making it difficult for countries to rationally prioritize prevention strategies. WHO and a global network of experts therefore undertook a collaborative effort to address this, with the aim of developing this simple data collection tool. The resulting Global Burn Registry (GBR) has been finalized following extensive pilot testing which took place in 60 health facilities across 30 countries.

Results: The GBR is based on an easy-to-use data collection form that is used for patients with burns who are admitted to a health facility. It takes approximately 5 minutes to complete. Data can be accessed and exported through the online registry interface maintained by WHO. The data collection form, as well as the online interface, are available in English, French and Spanish. The online interface allows users to view data from their health facility as well as all other participating health facilities. Data from the GBR (which are stripped of reference to information which allows identification of individual patients) are publicly available, and can be accessed and exported for further analysis by researchers and policy- makers, among others. The online platform also provides extensive data visualization and filtering capabilities.

Conclusion: The GBR has over 1300 records entered, with monthly uploads of 200-250 cases. Access to the GBR is available to the public at https://www.who.int/violence_injury_prevention/burns/gbr/terms/en/. 
O13.03 The epidemiology and prognosis of patients with massive burns: A multicenter study of 2483 cases

\section{CA Shen}

The Fourth Medical Center of Chinese People's Liberation Army General Hospital, BEIJING, China

Objectives: Epidemiological features of massively burned patients in our country remain unclear. This study was designed to investigate the epidemiological characteristics and evaluate the burn index (BI) and other risk factors associated with the prognosis of massively burned patients.

Methods: The data of 2483 patients with $\geq 30 \%$ total body surface area admitted in 2014 were retrieved from 106 burn centers in our country. Information on epidemiological features and the outcome were collected for retrospective analysis.

Results: Approximately $17.76 \%$ of patients were admitted to the local burn center after 6 hours post-burn injury. The mortality was $9.79 \%$, and the area under the receiver operating characteristic curve for BI was 0.941 . The mortality increased significantly when the value of BI was above a threshold of 29 in the 0-14 year group, 43.5 in the 15-59 year group and 35.5 in the $\geq 60$ year group. Multivariate logistic regression analyses showed that the odds ratio of death increased by $6 \%$ with an increase in the BI of 1.0. Patients aged 50 years or above, admitted over 6 hours post-injury and patients with a combined inhalation injury were at a higher risk of death.

Conclusion: The etiological characteristics of the different age groups should be considered for prevention. BI can be regarded as a reliable prognostic factor in severe burn patients. The results showed that a large BI, elderly age, delayed admission post-injury and combined inhalation injury are the main risk factors for extensive burn patients.

Picture $\quad 1: \quad$ https://www.eventure-online.com/parthenuploads/89/9EBA/add_1_540710_556b8ae2-6694-4707-ba18-ed7c0e67b58b.png

Caption 1: Figure - Cutoff determination of BI in different age groups

O13.04 Outpatient treated infant burns: epidemiological survey and prevention planning

R.T. Tatar, S. Stoicescu, D.M. Enescu

1Grigore Alexandrescu Clinical Emergency Hospital for Children, BUCHAREST, Romania

Objectives: Burn injuries represent a highly aggressive trauma for the human being, especially when the victim is very young. Considering the relative lack of data regarding burns in infants, we focused on this special age group. The children's development during the first year of life when they are almost completely dependent on others, justifies a more focused approach for describing burns circumstances and impact in this population.

Methods: We performed a 6 years retrospective study in our Plastic Surgery and Burns Department in Bucharest for identifying burn infants aged up to 12 months and treated as outpatients. The main goal was to achieve an epidemiological survey in terms of burn etiology, location, depth and TBSA involved, the parental attitude (described as the time elapsed before seeking specialized medical advice) and burn wound management. The secondary goal is to establish scientific grounds for a prevention campaign.

Results: Between 2012-2017 we found 464 patients meeting the inclusion criteria, representing $7,84 \%$ of all burned patients seen in our department in this time. Most patients were male, and the most frequent etiology was scalds, followed by contact burns. TBSA, burn depth and location and time elapsed from burn until we examined the patient were also recorded and analyzed.

Conclusion: Although burns in infants usually do not involve large surfaces and are partial thickness burns, they have to be approached as seriously as any other burn. We found an important group at risk of suffering these highly preventable injuries. Prevention measures should emphasize having the infant sleep or play in safe places and always under an adult's watch, and also not handling hot objects, liquids or food while carrying the baby. For the best outcome, the infant should be seen and treated by a burn specialist as soon as possible.

O13.05 Senna laxative-induced severe chemical burns in children 
L GOFFINET, L FLEURENTIN, N PETITPAIN, L JAVOT, S ADETU, L MARTRILLE, JL SCHMUTZ, AC BURSZTEJN

CHRU Nancy, VANDOEUVRE-LES-NANCY, France

Objectives: Senna is a natural laxative used in constipation. The aim of these case-reports is to inform of the potential side effects of senna in children.

Methods: We report 2 pediatric cases of acute erosive lesions after the consumption of herbal tea containing senna.

Results: Case 1: A 3 years old boy was hospitalized for a diaper rash with blisters. Physical examination showed typically diamond-shaped lesions involving the whole buttocks. There was a setting of abundant diarrhea since one day. Biological results were in the normal range. Bacteriological cultures were negative. After an extensive questioning, the father admitted that, the day before, his son had drunk herbal tea containing senna. The injured skin was treated with airexposure and chlorhexidine tanning and healed within 8 days, leaving demarcated erythema.

Case 2: A 14 months old child experienced diaper rash with well-limited diamond-shaped erythema of the buttocks associated with large erosions and blisters, and few necrotizing lesions of the epidermis on the left buttock. Skin lesions had linear demarcation closely aligned with the diaper edge and the gluteal fold was spared. Biological results were normal. The family reported the prescription to the child by a phytotherapist of senna tea (Mediflor ${ }^{\circledR} n^{\circ} 7$ ) for chronic constipation during 8 days before the lesions onset $(720 \mu \mathrm{g}$ of senna per day). The lesions were treated with airexposure and chlorhexidine tanning. The evolution was marked by a superinfected necrosis on the left buttock, requiring surgical drainage and the skin healed few days later, leaving light well demarcated erythema.

Conclusion: Senna administration in children can lead to buttock dermatitis generating severe pain and wrong accusation of child abuse and scars. In our 2 cases, child abuse case was indeed initially suspected. It is important to be aware of senna contraindication in children.

Picture $\quad 1: \quad$ https://www.eventure-online.com/parthenuploads/89/9EBA/add_3_541802_17180644-91ac-499a-9a63-86f432747885.png

Caption 1: Clinical pictures of Case 1, diamond-shaped demarcated inflammatory erosions and flaccid blisters on buttocks due to senna laxative

Picture 2: $\quad$ https://www.eventure-online.com/parthenuploads/89/9EBA/add_1_541802_17180644-91ac-499a-9a63-86f432747885.png

O13.06 Thermal Injury caused by Warmed Saline Irrigation During the Microvascular Free Flap

JH Yang

Chonbuk National University College of Medicine, JEONJU-SI, CHONBUK, South Korea

Objectives: Thermal injury to free flap site can be severe and distressing injuries both for the patients and the surgeon. Thermal injuries to insensate free flaps are known complications often reported. But thermal injuries to free flaps are also occurred in the perioperative period. This study focused on the warmed saline irrigation associated with this injury.

Methods: A 36-years old male patient initially presented to the hospital with diabetic foot ulcer on dorsum of left foot. After several debridement of necrotic tissue, and an anterolateral thigh perforator free flap was performed. The massive warmed saline irrigation was applied to the free flap site for dressing after skin suture. There were some changes on the flap such as heating sensation, redness and bullae formation after warmed saline irrigation (Fig. 1).

Results: The size of the burn was $3 \times 4 \mathrm{~cm}$, and the dressing was continued until 10 days postoperatively. Fortunately, there was no partial loss or defect of the flap. (Fig. 2, 3).

Conclusion: The most common causes of burns during surgery are burns due to heated material, direct injuries caused by IV bags, bottles and hot packs. The next is known as damage by various warming equipment. Additionally, warmed saline irrigation is often too hot and a temperature excess $48^{\circ} \mathrm{C}$ should be considered very hot saline during the free tissue transfers. So, surgeon must be aware that warmed saline irrigation can cause thermal injury to free flap during the free tissue transfer. 
Picture

1:

https://www.eventure-online.com/parthen-

uploads/89/9EBA/add_1_539302_6ddd15bf-9e17-4249-badb-778a3e252fe4.jpg

Caption 1: Immediately postoperative. During the warm He got burned and the redness and bullae formation was shown.

Picture

2:

https://www.eventure-online.com/parthen-

uploads/89/9EBA/add_2_539302_6ddd15bf-9e17-4249-badb-778a3e252fe4.jpg

Caption 2: Postoperative 2 days. A patient sustained a partial thickness injury to the foot.

Picture 3: https://www.eventure-online.com/parthen-uploads/89/9EBA/add_539302_6ddd15bf9e17-4249-badb-778a3e252fe4.jpg

Caption 3: Postoperative 10 days. The flaps healed uneventfully with daily dressing after 10 days.

O13.07 Elderly burn injuries and understanding the common causes to influence accident prevention

\section{N Chana, J Smith}

University of Leeds, LEEDS, United Kingdom

Objectives: To evaluate the causal factors of elderly burns admitted to Pinderfields General Hospital, in order to understand how this can influence accident prevention in the future.

Methods: Patients were aged 65 years or older and had to have been admitted for a minimum of one night to the regional burns unit at Pinderfields General Hospital from January 2012. The data was collected from the International Burn Injury Database (iBID), which included 442 patients over 65 years old. The data was analysed using descriptive analysis.

Results: $13.0 \%$ of all patients admitted with a burn injury were elderly. Burn injuries were most common during the activity of food preparation with a proportion of $31.2 \%$ of all burn injuries being accountable to this activity. Out of all the burn injuries within food preparation, $75.4 \%$ were due to a scald injury. Additionally, 42.3\% of scald burns from food preparation were due to hot fluid spills from a kettle or saucepan, rising to $73.1 \%$ after including burns from cups of tea and coffee. 21.2\% of scalds from food preparation were caused by cooking with hot oil.

Conclusion: The main cause of burn injuries in the elderly of Yorkshire and Humber was food preparation. The majority of the food preparation burns were a scald burn due to the handling of hot fluids, either from a saucepan or a kettle. A prevention strategy aiming to make people aware of this finding can help reduce burn injuries in the elderly. We recommend that these findings should be shared with elderly care charities to help tailor accident prevention measures for people in their homes. More research needs to be performed to understand if these findings reflect the nature of burn injuries within elderly patients nationally.

\section{O13.08 From 'Young Burn Survivors Day' to a 'World Burn Awareness Day'}

\section{A. E. Gottwald}

Paulinchen - Initiative for Young Burn Survivors, NORDERSTEDT, Germany

Objectives: Imagine if we could have one day each year when we all work together to increase worldwide awareness of the high number of burn accidents, and educate and warn about safety hazards, and provide information about first aid procedures and about the serious consequences of burns and scalds.

Methods: In December of 2010, the German organisation Paulinchen - Initiative for Young Burn Survivors created the national "Young Burn Survivors Day". Since then, various events have been organized by hospitals, pharmacies, fire fighters, kindergartens and schools on December $7^{\text {th }}$ each year, in order to increase awareness of burn risks and prevention methods. In 2018, we had 136 events including 8 international partners.

Results: In the UK there is a National Burn Awareness Day in October. On the website one can find a brilliant toolkit for download. And the hashtag \#BeBurnsAware, for example, has been viewed by 2.1 million people. There are more National Burn Awareness days and weeks in different countries each year. 
Conclusion: Our goal is to bring all of these world wide activities together to implement a "World Burn Survivors Day" which would be listed and supported by the WHO. We suggest establishing a worldwide platform where all information about the existing National Burn Awareness Days and the informational materials are collected and accessible, so that everyone can learn from one another and use and exchange ideas and materials.

O14.01 Recommendations on the most suitable health related quality-of-life measurement instrument after burn injury: a systematic review of measurement properties

CM Legemate $^{1}$, I Spronk ${ }^{1}$, LB Mokkink², E Middelkoop³, S Polinder, M.E. Van Baar ${ }^{1}$, CH Van der Vlies ${ }^{5}$

${ }^{1}$ Maasstadziekenhuis, ROTTERDAM, Nederland

${ }^{2} \mathrm{VU}$ university, AMSTERDAM, Nederland

${ }^{3}$ Amsterdam UMC, AMSTERDAM, Nederland

${ }^{4}$ Department of Public Health, Erasmus MC, University Medical Center Rotterdam, ROTTERDAM, Nederland

${ }^{5}$ Erasmus Medische Centrum, ROTTERDAM, Nederland

Objectives: To critically appraise the quality of existing patient-reported outcome instruments developed and/or validated for health related quality of life (HRQL) measurement in patients after burn injury.

Methods: A systematic literature search was conducted in Embase, Medline, CINAHL, Cochrane, Web of Science and Google scholar to identify studies on measurement properties of HRQL instruments used in patients after burn injury. Measurement properties of identified instruments were assessed using the COnsensus-based Standards for the selection of health Measurement INstruments (COSMIN) checklist. A modified GRADE analysis was performed to facilitate recommendations for future use of HRQL instruments after burn injury.

Results: Forty-two studies covering 12 HRQL instruments (9 disease-specific instruments and 3 generic instruments) were included. The BSHS-B was the instrument most assessed and demonstrated positive quality evidence for most measurement properties but lacked evidence for sufficient content validity. Of all instruments, the adult version of the BBSIP demonstrated the strongest evidence for content validity but lacked evidence on other measurement properties.

Conclusion: This is the first systematic review to identify and critically appraise HRQL instruments in burn patients using internationally accepted criteria. The disease-specific BSHS-B possessed the strongest evidence for sufficient quality of measurement properties and is therefore recommended as the most suitable instrument, although future studies on content validity are necessary. The adult version of the BBSIP was the only instrument that met the high standards for high-quality PROM development and content validity and has, therefore, a high potential to be recommended if further studies on other measurement properties show positive results.

\section{O14.02 BQIP: Improving Burn Care Through Registry Data}

$\underline{\text { Y Singer }}^{1}$, L Tracy ${ }^{2}$, B Gabbe ${ }^{2}$, P Cameron², T Perrett ${ }^{3}$, J Gong'2 ${ }^{2}$ F Wood ${ }^{4}$, H Cleland ${ }^{5}$

${ }^{1}$ Victorian Adult Burn Service, PRAHRAN, Australia

${ }^{2}$ Monash University, MELBOURNE, Australia

${ }^{3}$ National Burn Centre, AUCKLAND, New Zealand

${ }^{4}$ Burn Service, WA, PERTH, Australia

${ }^{5}$ Victorian Adult Burn Service, The Alfred, MELBOURNE, Australia

Objectives: Research and advances in treatment have contributed to significant improvements in burn care and the quality of survival. Nevertheless, significant evidence gaps remain and high variations in clinical practice exist; the effects of which are largely unknown. The aim of this study is to quantify variation in practice in the management of burn injury between Australia and New Zealand burns centres and establish whether this practice variation has significant impact on clinical and patient outcomes. 
Methods: Data were extracted for all acute admissions to the 17 Australian \& New Zealand specialist burns centres between 2009 and 2017 from the Burn Registry of Australia and New Zealand (BRANZ). The BRANZ is a clinical quality registry that collects epidemiological, structural, process and outcome data on patients admitted to Australian and New Zealand burns centres.

Results: Of the 23,796 cases, they were predominantly adults $(68 \%)$ and male $(68 \%)$. Scalds and flames accounted for approximately $70 \%$ of injuries, and more than $80 \%$ of patients had a\%TBSA of $<10 \%$. Inhalation injury was rare (5\%). Almost $75 \%$ of cases underwent a surgical procedure. Only $1.2 \%$ of all cases died.

Wide variations in practice were identified across burns centres in patients in several areas of clinical practice. These included the administration of venous thromboembolism prophylaxis, and the commencement of enteral nutrition in less than 24 hours for patients with burns $>20 \%$ TBSA.

Conclusion: This presentation will describe some of the variation in quality indicator performance across Australian and New Zealand burn centres and analyse the effects of variation on inpatient outcomes. Identifying and understanding the effects of variation in practice can drive quality improvement initiatives which have much potential to improve burn care and patient outcomes in the region.

O14.03 The Development of a Norwegian Burns Registry

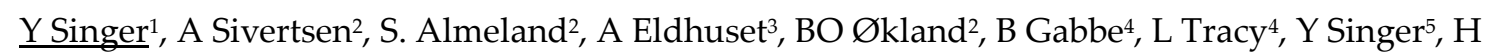
Cleland $^{5}$

${ }^{1}$ Victorian Adult Burn Service, PRAHRAN, Australia

${ }^{2}$ Haukeland University Hospital, BERGEN, Norway

${ }^{3}$ Central Norway Regional Health Authority, TRONDHEIM, Norway

${ }^{4}$ Monash University, MELBOURNE, Australia

5Victorian Adult Burn Service, The Alfred, MELBOURNE, Australia

Objectives: In recent decades, research and advances in treatments have contributed to significant improvements to burn care and the quality of survival. Nevertheless, significant evidence gaps and high variations in clinical practice remain, the effects of which are largely unknown. Clinical quality registries allow for the routine collection and analysis of burn-related information that allows for identification of benchmarks and variation in clinical outcomes to close knowledge gaps and inform best practices. We present a newly established medical quality registry for treatment of major burn injuries in Norway.

Methods: The process of planning, establishing, and implementing the registry in close collaboration with the Burns Registry of Australia and New Zealand (BRANZ) is presented. We present the legal formalities and regulations for handling and storing data in the registry and discuss contemporary and future benefits of a national quality registry.

Results: The registry recruits patients referred to the national burn center at Haukeland University Hospital in Bergen. At each point along the pathway of care, starting at the site of injury, all surgical procedures and any comorbidity are recorded in an online computer system.

Conclusion: The main purpose of the registry is to enable an evaluation of procedures and treatment outcomes, patients' satisfaction, burden of care, and to provide a platform for future clinical research.

O14.04 Development of a risk prediction model (Hangang) and comparison with clinical severity scores in burn patients

\section{IC Yoon}

Hangang Sacred Heart Hospital, SEOUL, South Korea

Objectives: The purpose of this study was to develop a new prediction model to reflect the risk of mortality and severity of disease and to evaluate the ability of the developed model to predict mortality among adult burn patients.

Methods: This study included 2009 patients aged more than 18 years who were admitted to the intensive care unit (ICU) within 24 hours after a burn. We divided the patients into two groups; those 
admitted from January 2007 to December 2013 were included in the derivation group and those admitted from January 2014 to September 2017 were included in the validation group. Shrinkage methods with 10 -folds cross-validation were performed to identify variables and limit overfitting of the model. The discrimination was analyzed using the area under the curve (AUC) of the receiver operating characteristic curve. The Brier score, integrated discrimination improvement (IDI), and net reclassification improvement (NRI) were also calculated. The calibration was analyzed using the Hosmer-Lemeshow goodness-of-fit test (HL test). The clinical usefulness was evaluated using a decision-curve analysis.

Results: The Hangang model showed good calibration with the HL test $(\chi 2=8.785, \mathrm{p}=0.361)$; the highest AUC and the lowest Brier score were 0.943 and 0.068 , respectively. The NRI and IDI were 0.124 $(\mathrm{p}$-value $=0.003)$ and $0.079(\mathrm{p}$-value $<0.001)$ when compared with FLAMES, respectively .

Conclusion: This model reflects the current risk factors of mortality among adult burn patients. Furthermore, it was a highly discriminatory and well-calibrated model for the prediction of mortality in this cohort.

O15.01 Silkworm silk as an alternative to Biobrane and Suprathel for the treatment of acute superficial burns

\section{Schiefer, PC Fuchs}

Hospital Cologne Merheim, COLOGNE, Germany

Objectives: During the treatment of superficial burn injuries patients are often confronted with painful dressing changes. Therefor we usually treat these wounds with materials like Suprathel ${ }^{\circledR}$ or Biobrane $^{\circledR}$ that can stay on the wound and slowly peel off during re-epithelialization. Since 2013 we started using pure silk as cost efficient alternative for the treatment of these wounds. Therefore we wanted to compare it directly with the other dressings.

Methods: In a prospective intra-individual design we evaluated the wound healing of 50 patients with superficial burn injuries. Hereof 30 patients received both silk and Biobrane and 20 further patients received silk and Suprathel for the treatment of their burn injuries. Following a standardized case report form, we monitored pain, active bleeding, exudation, dressing change and inflammation. Furthermore we evaluated scar quality objectively with the Cutometer, Mexameter and Tewameter as well as subjectively with the patient and observer scar scale (POSAS) and the Vancouver scar scale (VSS).

Results: During wound healing no significant differences could be found regarding wound closure, inflammation and acute bleeding. Pain levels fell fast during after dressing application and during wound healing. Long-term results in functional and aesthetic outcome were similar. High satisfaction rates were reported for all dressings, with different individual preferences.

Conclusion: All three dressings provided a safe and effective healing environment, with low complication rates in regard to infection and exudation. Additionally, no significant differences regarding scarring could be found with an overall pleasing aesthetic outcome. Therefore silk is a cost efficient alternative for the treatment of superficial burn injuries.

O15.02 Dielectric profiles at microwave frequencies of different burn depths: Preliminary data from ex-vivo human (burnt) skin samples

M.D. Perez ${ }^{1}$, J Ebrahimizadeh ${ }^{1}$, KB Pramod ${ }^{1}$, SRM Shah ${ }^{1}$, I El-Ali², M Kouki ${ }^{2}$, F Huss ${ }^{3}$, R Augustine $^{1}$

${ }^{1}$ Uppsala University, UPPSALA, Sweden

${ }^{2}$ Datametrix AG, NEUCHÂTEL, Switzerland

${ }^{3}$ Uppsala University Hospital, UPPSALA, Sweden

Objectives: "Senseburn" (http://www.senseburn.com) is a MedTech research project funded by the European Union's program Eurostars to develop an advanced burn diagnostic tool for clinical use. A non-invasive microwave sensor and a software system to estimate and document the depth and extent of human burns on a 3D body model are the main foreseen innovations. Currently, as part 
of the research and development, dielectric spectroscopic characterization at microwave frequencies of ex-vivo human samples is carried out. This abstract presents preliminary results.

Methods: The Senseburn consortium conducts a clinical trial campaign in Uppsala University Hospital's Burn center (Sweden) to collect human tissue samples (surgical waste) corresponding to different burn depths, from full thickness to superficial burns. After informed consent, samples are collected in the OR during necrosectomy of acute burns and sent to The Angstrom Laboratory for microwave dielectric spectroscopy together with the clinical assessment of the burn depth. Samples are divided into homogeneous sectors and dielectric profiles at microwave frequencies are measured. Sectors consist of different measurement points (repetitions) for better homogenization of reported values. Measured dielectric profiles are compared to those reported in the Italian IFAC-CNR database as is the standard deviation to consider the accuracy of the measurements.

Results: In keeping tissue degradation to a minimum the lead-time between excision and measurements were kept to a minimum (mean 10 hours). Compared to the IFAC-CNR database the dielectric profile of sectors corresponding to superficial dermal burns are found to be close to the typical dry skin values. Deeper injuries present lower dielectric profiles reaching values similar to those corresponding to bone and fat.

Conclusion: These preliminary results show a positive suggestion to proof-of-concept: It is possible to distinguish different burn depths by their respective dielectric profiles. However, more data need to be collected to enhance statistically the reliability of these findings.

O15.03 A highly sensitive aspiration device for assessment of scar stiffness in pediatric burns

\section{LElrod $^{1}$, B Mueller'2, M Meuli' ${ }^{1}$, E Mazza ${ }^{2}$, C. Schiest ${ }^{1}$}

${ }^{1}$ Pediatric Burn Center, University Children`s Hospital Zurich, ZURICH, Switzerland

2Institute for Mechanical Systems, ETH Zurich, ZURICH, Switzerland

Objectives: As to improve burn surgery and scar rehabilitation, objective evaluation of scar quality is crucial, in addition to clinical assessment of burn scars. A number of devices for objective assessment of mechanical properties of skin, such as for example suction-based tools are available. However, these tools are limited by their intra- and inter-observer variability on the one hand and by their moderate practicability especially in pediatric patients on the other hand. A novel suction device, utilized in the present study, the Nimble, is conceived to overcome these limitations.

Methods: The new device Nimble consists of an ultra-light suction probe that measures the negative pressure needed to obtain a predefined tissue elevation, thereby revealing information on its stiffness. 29 pediatric patients with a burn scar were included into the study. 3 blinded observers measured tissue stiffness on defined locations on the scar and on the adjacent healthy skin using the established Cutometer ${ }^{\circledR}$ (control) and the novel device (test). Ability and reliability of both instruments to distinguish between healthy skin and scar was assessed by means of the intraclass correlation coefficient (ICC). In a second stage, scar maturation was assessed by means of repetitive measurement over time.

Results: Regarding tissue stiffness, the Cutometer ${ }^{\circledR}$ successfully differentiated between scar and healthy skin in $80 \%$, the novel device in $92 \%$ of the patients ( $p<0.05$ ). Inter- and intra-observer variability of the Nimble (ICCs) were excellent. The Nimble exceeded the Cutometer ${ }^{\circledR}$ for the majority of the calculated ICC values. Furthermore, measurements were less susceptible to patient noncompliance and observer dependency.

Conclusion: Both devices are highly competent to distinguish between scar and healthy skin. However, the Nimble appears more sensible and easier to handle and might, therefore, be more suitable for clinical practice and research.

O15.04 Equivalence and feasibility of the paper-based and electronic versions of the POSAS, the EQ-5D and the DLQI in a Belgian scar population

L Meirte $^{1}$, N Hellemans ${ }^{1}$, M Anthonissen², T Vanhullebusch², K Maertens², P Moortgat ${ }^{2}$, U Van Daele $^{1}$

${ }^{1}$ University of Antwerp, WILRIJK, Belgium 
${ }^{2}$ Oscare After-care and research centre for burns and scar, ANTWERP, Belgium

Objectives: Patient reported outcome measures (PROMs) are crucial within patient-centred care. The use of electronic PROMs (ePROMs) is increasing and multiple advantages of electronic data collection has been described. The goal of this research was to compare the paper versions of the Patient and Observer Scar Assessment Scale (POSAS), the Euroqol 5 Dimensions (EQ-5D-5L) and the Dermatology Life Quality Index (DLQI) with a web-based version of the measures. We assessed equivalence of scores, the differences in completion time, data processing time and patients' preferred mode and ease of use.

Methods: We used a randomized crossover design using a within-subject comparison of the formats of the questionnaires. International Society for Pharmacoeconomics and Outcome Research (ISPOR) guidelines were followed. Participants aged over 18 with a scar were recruited from Oscare, an outpatient after-care and research centre for scars and burns located in Antwerp, Belgium.

Results: Twenty patients with scars participated of which 14 were burn scars. Paired sample ttests indicated that completion time between the paper and electronic POSAS and DLQI were not significantly different. For the electronic EQ-5D the time to complete was significantly shorter $(\mathrm{p}<.05)$. For the 3 questionnaires significant difference in data processing time $(p<.005)$ was found in favour of the ePROMs. Eighteen out of 20 participants preferred the electronic mode of administration and found the electronic administration easy to use. Results of equivalence testing will be presented at the conference.

Conclusion: In conclusion, data processing time for ePROMs is significantly reduced when using these ePROMs, moreover participants prefer electronic formats and find it easy to use. This study gives encouragement to the further widespread development and use of ePROMs within the burn and scar population.

O15.05 Effect of Platelet-Rich Plasma Application On Healing of Donor Site Wounds of Partial Thickness Skin Graft

\section{H O Sheriff $^{1}$, B K Toufik ${ }^{2}$}

${ }^{1}$ Sulaimani University, College of Medicine, SULAIMANIAH, Iraq

${ }^{2}$ Rzgary hospital, ERBIL, Iraq

Objectives: The aim of this clinical trial was to study the effect of topical Application of single stage autologous platelet-rich plasma on the healing of donor sites wound of split thickness skin graft.

Methods: Fifteen patients were older than 15 years (median age, 36 years) were enrolled for this study. Two adjacent donor site wound or contralateral donor site wound of the same anatomical position of the same patient that was made by electrical dermatome of the same depth. One donor wound application of platelet-rich plasma done, other site left without application of platelet-rich plasma (control). Biopsy specimens $(4 \mathrm{~mm})$ from donor wounds were evaluated for neo-epidermal coverage in tissue section in day eight. Macroscopic healing and pain at dressing removal were assessed.

Results: Healing of the donor wounds differs between platelet-rich plasma and control on day five or day eight. A great difference in macroscopic epithelialization between platelet-rich plasma and control were found for donor wounds. Pain off dressing showed lower levels of pain score (1$10)$, platelet-rich plasma(mean:4.42) and control donor wounds (mean: 6.28).

Conclusion: Macroscopic epithelialization of donor wounds was remarkably influenced by platelet-rich plasma treatment.

Picture $\quad 1: \quad$ https://www.eventure-online.com/parthenuploads/89/9EBA/add_1_545723_823d2682-af06-446c-8dca-642021c9ecdc.jpg

Caption 1: Day 8 , result of two skin graft donor site, Rt thigh with PRP application, and the Lt thigh without PRP.

O15.06 Developing a patient portal to facilitate shared decision making with burn patients during their recovery: current status and key learnings 
G.A. Wildenbos $^{1}$, T. Haanstra ${ }^{1}$, T. Radstake ${ }^{2}$, M. Van Baar ${ }^{3}$, N. Van Loey ${ }^{4}$, E. Esther ${ }^{5}$, M. Nieuwenhuis, ${ }^{6}$ A. Pijpe ${ }^{7}$, C. Van Schie ${ }^{8}$, S. Scholten ${ }^{9}$, C.H. Van der Vlies ${ }^{10}$, P. Van Zuijlen ${ }^{7}$

${ }^{1}$ Dutch Burns Foundation, ADBC, BEVERWIJK, Netherlands

${ }^{2}$ Association for people with burns, BEVERWIJK, Netherlands

${ }^{3}$ Association of Dutch Burn Centers, Maasstad Hospital, ROTTERDAM, Netherlands

${ }^{4}$ Association of Dutch Burn Centers, BEVERWIJK, Netherlands

${ }^{5}$ Association of Dutch Burn Centers, Amsterdam UMC, Vrije Universiteit Amsterdam, BEVERWIJK, AMSTERDAM, Netherlands

${ }^{6}$ Association of Dutch Burn Centers, Martini Hospital, GRONINGEN, Netherlands

${ }^{7}$ Burn Center, Red Cross Hospital, BEVERWIJK, Netherlands

${ }^{8}$ Dutch Burns Foundation, BEVERWIJK, Netherlands

${ }^{9}$ Burn Center, Martini Hospital, GRONINGEN, Netherlands

${ }^{10}$ Burn Center, Maasstad Hospital. Erasmus MC, University Medical Center Rotterdam, ROTTERDAM, Netherlands

Objectives: The goal of the 'Aftercare portal', a digital patient portal, is to facilitate patients and healthcare professionals in shared decision making (SDM) regarding treatment options after a patient has been discharged from a Burncentre. This includes scar treatment as well as interventions regarding other physical and psychosocial aspects that a patient may encounter during his/her recovery. This portal will be implemented at the three Dutch Burncentres at the start of 2020.

Methods: The development of the Aftercare portal started in September 2018 and finds its foundation in scientific knowledge on the development of eHealth as well as SDM. Based upon experience in eHealth co-creation with patients, we address our approach in reaching the milestones during the first year of the portal's development. We further highlight the main steps regarding the implementation of the portal at the three Dutch Burncentres.

Results: Since the portal is in development, a prototype of the portal is shown during the EBA in September 2019. The presentation shows key learnings throughout its development process so far, specifically focusing on learnings regarding the development of a decision aid for patients regarding scar treatment. For example, we highlight the importance of involving diverse end-users and creating content in the portal that suits patients with lower health literacy levels. In addition, we share our experiences with co-creation methods in which patients are crucial advisors to the development of the portal.

Conclusion: In reaching its goal to facilitate patients and healthcare professionals in SDM, key elements in the development of the portal are to create information and interactions that suits the needs of burns patients as well as the work processes of the healthcare professionals. Conclusions on key-learnings regarding the development and co-creation process on this matter will evolve over the next months and will be given during the presentation.

O15.07 Nanografts to enhance epthelialization

\section{IVuola}

Helsinki University Hospital, ESPOO, Finland

Objectives: The Meek graft technique makes it possible to expand skin grafts up to 10 fold. The grafts must be orientated correctly to enable vascularization and graft take. It has been shown by a Harvard group that if a split thickness skin graft is minced to pieces of $0.8 \mathrm{~mm}$ they can be spread to the wound without considering their orientation. We have developed further this technique and minced the grafts even in smaller pieces and show here the preliminary clinical results in burn patients.

Methods: Small split thickness skin grafts, $5 \times 5 \mathrm{~cm}$, were minced with a special blade tool into skin peaces smaller than $0.8 \mathrm{~mm}$. The pieces were spread onto 1:1 meshed allografts on their inner surface. The allografts were applied on top of widely meshed autografts and the epithelialization was compared to similarly grafted sites without any minced grafts. Another method was to spread the nanografts to the donor site or to the wound bed before applying 1:6 meshed Meek grafts.

Results: The pilot study shows promising results in epithelialization rate. Very small skin pieces not usable for normal meshing could be minced and applied. 
Conclusion: In this pilot study the very small split thickness sklin pieces minced smaller than 0.8 $\mathrm{mm}$ could be applied to the wound bed without considering their orientation and showed promising results in enhancing epthelialization rate.

O16.01 Systemic antibiotic prophylaxis does not improve the outcome of burn injuries in children: a meta-analysis

IG Jozsa ${ }^{1}$, CSA Csenkey ${ }^{1}$, VP Vajda ${ }^{1}$, HP Hegyi², GA Garami²

${ }^{1}$ Department of Pediatrics, Surgical Unit, PÉCS, Hungary

2Institute for Translational Medicine, Medical School, University of Pecs, PÉCS, Hungary

Objectives: In pediatric burns the use of systemic antibiotic prophylaxis is a standard procedure in some burn centers, though its beneficial effect on the infectious complications is debated. We aimed to determine whether systemic antibiotic prophylaxis prevents infectious complications in children with burn injuries by using meta-analysis.

Methods: We searched the PubMed, EMBASE, and Cochrane Library databases from inception to May 2018. We included 6 studies, in which event rates of infectious complications were reported in children with burn injuries receiving or not receiving systemic antibiotic prophylaxis.

Results: We found that the chances for local (odds ratio, OR=0.99; $95 \% \mathrm{CI}, 0.40,2.47$ ) and systemic (OR=0.74; 95\% CI, 0.38, 1.45) infectious complications were not different between antibiotic-treated and non-treated children with burn injuries. The overall chance for infections was also not different between the groups (OR=1.35; 95\% CI, 0.44, 4.18). Based on age, affected total body surface area, and country income level, we did not find any subgroup that benefited from the prophylaxis.

Conclusion: Our findings provide quantitative evidence for the inefficacy of systemic antibiotic prophylaxis in the prevention of infections in pediatric burns. Well-designed, multinational, randomized trials are warranted to validate our findings in a diverse population of children with burn injuries.

O16.02 Nutrition in the Burn injured child: an audit

\section{E Bridge, $\underline{\text { S Pearce }}$}

Nottingham University Hospitals NHS Trust, NOTTINGHAM, United Kingdom

Objectives: In 2016, Nottingham Children's Hospital (NCH) produced "Nutrition in the Burn Injured Child" guidelines. To determine adherence to guideline recommendations an audit of nutritional care delivered to burn injured children at $\mathrm{NCH}$, over a 1 year period, was conducted.

Methods: In October 2018, case notes of all burn injured children admitted between 01/09/1731/08/18 were reviewed. Audit questions were set based on the clear practice recommendations made in the trust nutrition guidelines and used for data collection for all case note reviews. Data analysis determined percentage adherence to each recommendation for the whole sample.

Results: 113 patients (62 males: 51 females) were identified with a mean age of 4.4 years, average burn size of $4.6 \%$ and average length of stay of 2.4 days. Complete nutritional screening (weight \& height/length) within 24 hours of admission was completed in only $8 \%$ of patients, though $99 \%$ of patients had their weight measured within this time frame. No patients had their weight or height plotted on growth chart centiles. $23 \%$ of all patients met dietetic referral criteria but only $66 \%$ of these were referred to the dietetic service. Dietetic assessment was undertaken within the recommended time in $100 \%$ of those referred but only $77 \%$ of patients seen had dietetic plans documented in medical notes. No patients, who met guideline criteria, had a full biochemistry screen. $4 \%$ of patients met enteral feeding criteria but, despite 2 patients having NGTs placed, none received enteral feeding. No patients met guideline criteria for nasojejunal tube feeding and only $21 \%$ of patients who stayed overnight had their bowel movements monitored.

Conclusion: Several aspects of NCH burn nutrition care can be improved upon. Audit results will be used to inform future staff education, guideline development and dietetic practice going forward and a re-audit is planned for 2019. 
O16.03 Factors affecting length of stay among pediatric and adult patients admitted to the Lebanese Burn Center: a retrospective study

\section{G G GHANIMÉ, J B Bourji}

Lebanese University, BEYROUTH, Lebanon

Objectives: Burn injuries are serious lesions requiring specialized medical care and associated with prolonged length of hospital stay (LOS). This study aims to elucidate the epidemiological and clinical factors affecting the LOS of pediatric and adult patients presenting with burn wounds.

Methods: A single-center retrospective study was conducted in the Hopital Libanais Geitawi Burn Center in Lebanon. Medical records of patients admitted to the burn center between January 2014 and December 2018 were retrieved. Epidemiological and clinical data, such as age, gender, LOS, comorbidities, as well

Results: 321 adult and 154 pediatric patients meeting the inclusion criteria were identified. Mean LOS in the total population was 23.58 days. Univariate analysis revealed inconsistent correlations between the studied factors and the LOS of pediatric and adult patients. Factors positively affecting both populations were: undergoing an operation, the number of operations, burn degree, infection, transfusion and requirement for burn excision and grafting. Additionally, among pediatric patients, LOS significantly increased with age, total body surface area (TBSA) of burn wound, cause of burn, sepsis, wound dressing under anesthesia, and escharotomy. On the other hand, female gender and fever were significant additional positive influencers of adult LOS. Multivariate analysis showed that both pediatric and adult LOS was significantly associated to the number of operations, transfusion, and requirement for burn excision and skin grafting. Adult LOS was further affected by age, sex, caloric intake and infection.

Conclusion: Our study demonstrated the differential influence of epidemiological and clinical factors among adult and pediatric populations, which allows better prediction of LOS and management of patients presenting with burn injuries.

O16.04 Interdisciplinary management of non-accidental burns in children: diagnostics and treatment in a pediatric burn centre

\section{E Renkert, S Krickeberg, K Maurer, B Lange, LM Wessel}

University Mannheim Medical Centre, MANNHEIM, Germany

Objectives: Non-accidental thermal injuries of infants and toddlers occur throughout all social classes with an incidence of up to $10 \%$ and an additional high estimated number of unrecorded cases. When suspicion arises, midwives, pediatricians and other medical personnel are obliged to work together with the local youth welfare office and forensic medical experts to set the course of action and protect the child's welfare. Uncertainty concerning the means of intervention is a common occurrence.

Methods: Since 2007, a child protection group, consisting of an interdisciplinary team of pediatric surgeons, pediatricians and child psychologists, has existed as part of the University Medical Centre Mannheim pediatric burn centre and is involved whenever the suspicion of acute child abuse arises.

Results: Using a case study, we demonstrate the standardized course of action of the child protection group within our pediatric burn centre, using the framework set by the S3-guideline on child abuse by the Association of the Scientific Medical Societies in Germany (AWMF) as well as the hospital's own SOP.

Conclusion: In Germany, nationwide structures are in place to offer professional assistance in cases of suspected child abuse. A child protection hotline for health care professionals offers consulting services for medical personnel 24/7 free of charge.

Cooperation with the local institutes of forensic medicine is an additional option to receive professional assessment and documentation of suspected cases.

A centralized coordination by the local child protection group has proven successful in effectively handling suspected child abuse cases and thereby ensuring the children's welfare. 
Y Shoham, N Sharon, Y Krieger, E Silberstein, T Ayzenberg, E Maor, A Douvdevani

Soroka University Medical Center, BEER SHEBA, Israel

Objectives: Despite great advances in the treatment burns, useful prognostic markers are sparse. There is increasing interest in circulating plasma cell free DNA (CFD) as a potential marker for tissue injury, however current methods for CFD analysis are impractical for routine laboratory use due to cost and complexity. We have developed a novel rapid direct fluorescent assay for CFD quantification that allows obtaining accurate, fast and inexpensive measurements, and already published its potential use in quantifying admission CFD levels as a prognostic factor in adult burns. The objective of this study was to explore the use of this technique for measuring admission plasma CFD levels as a prognostic factor in pediatric burns.

Methods: Plasma CFD levels are obtained at admission from otherwise healthy hospitalized pediatric burn patients, $0-18$ years old, within 24 hours of injury. DNA levels are quantified using the rapid direct fluorescent assay, and compared to various variables.

Results: To date 16 pediatric burn patients have been enrolled, 8 female and 8 male, aged $4.0 \pm 4.5$ years old. The average TBSA was $15.3 \pm 10.8 \%$, and average hospitalization was $15.6 \pm 10.2$ days. The average CFD level was $1747 \pm 1215 \mathrm{ng} / \mathrm{ml}$. There is a significant correlation between CFD levels and hospitalization days $\left(\mathrm{R}^{2}=0.31\right.$, AUC $\left.=0.854, \mathrm{p}=0.027\right)$. We did not find significant correlations with TBSA and burn depth, however, we found a significant correlation between the multiplication of the CFD levels by TBV (Total Burn Volume, a term previously described as TBSA*burn depth, either 2 for partial thickness, 3 for full thickness, and 2.5 for mixed depth) and hospitalization days $\left(\mathrm{R}^{2}=0.51\right.$, AUC $=0.98$, $\mathrm{p}=0.002$ ). There was also a significant correlation between CFD levels and the number of surgical procedures $\left(\mathrm{R}^{2}=0.6, \mathrm{p}=0.02\right)$.

Conclusion: Admission CFD levels may serve as a prognostic factor in pediatric burns. Larger patient groups are needed in order to further strengthen these results.

O17.01 The effect of moisturizers on scars: A systematic review

$\underline{\text { T Klotz }^{1}}$, Z Munn², E Aromataris², J.E. Greenwood ${ }^{1}$

${ }^{1}$ Royal Adelaide Hospital, ADELAIDE, Australia

2University of Adelaide, ADELAIDE, Australia

Objectives: A survey conducted revealed that 53 responding burn therapists recommended 29 different moisturisers, demonstrating a significant variability in practice. Further survey questions revealed the minimal use of any evidence to guide practice. Our objective was to systematically collect and collate all relevant evidence on the effect of moisturisers on scars. Synthesis of this data might then guide clinicians in both using, and recommending, moisturisers to best to obtain a desired outcome.

Methods: Keloid and hypertrophic scars were included, but atrophic scars were not. Databases searched included PubMed, CINAHL, Embase and Web of Science.

Results: In total, 1,570 studies were identified in the structured search. After removal of duplicates, screening and critical appraisal as per the published protocol, 33 studies were included. A meta-analysis (7 studies) could be conducted on the outcome of post-excision recurrence of keloid scars and the topical application of the immune modulator, Imiquimod. The remaining 26 studies were subjected to narrative synthesis and are presented according to outcomes. In addition, synthesis and grouping of the data into two groups; medicated creams and non-medicated (high cost and low cost) moisturisers assists with the presentation of clinically relevant information.

Conclusion: Product selection is usually based on cost, availability, lack of adverse events and the perceived ability to generate the outcome desired, often where evidence of efficacy is lacking. Very few moisturisers have been shown to impact on scar outcomes. There is a paucity of well-designed studies in this field, particularly in terms of outcome measures, which might enable strongly positive recommendations to be made.

O17.02 ICT as a tool in the rehabilitation of the burned patient: the Farmalarm APP

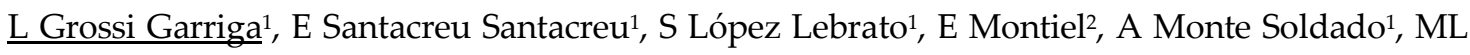
Torrent Bertran ${ }^{1}$ 
${ }^{1}$ Hospital Universitari Vall d'Hebron, BARCELONA, Spain

${ }^{2}$ VHIR - Vall d'Hebron Research Institute, BARCELONA, Spain

Objectives: Information and Communications Technology applications through a personalized multimedia environment are an indicated treatment when patients can't attend to on-site rehabilitation regularly.

Main objective is the adaptation and validation of a platform web suited to the needs of patients with burned hands. Farmalarm is a digital platform for Smartphone through which patients may receive information, display videos, check next medical appointments and contact professionals in case of doubt.

Methods: This is a validation study of the Farmalarm platform in consecutives patients with burned hands.

Five videos have been made focusing on the hands, working skin stretching for closing, opening, bimanual coordination, desensitization, and active movements for finger clamp. These videos were uploaded to Farmalarm.

Previous and on-site assessments were made, functionality with DASH, quality of life with EQ$5 \mathrm{D}$, and scar evolution with Vancouver Scale.

Then, we made a monthly on-site follow-up, with the same assessments, and a continuous followup on-line, through the APP, where patients can upload videos doing the exercises.

This platform allows us monitoring how many times they have been connected and the treatment adherence. Also chat and video call are available.

Results: As a main result, we get the adaptation of the generic platform Farmalarm with burned patients.

This project allows a follow-up of burned patients through a multimedia platform for rehabilitation, personalized and interactive. The rehabilitation team can program appropriate activities to the functional situation of the participants, and can modify it depending on the evolving observed in the regularly assessments planned.

This platform also allows us to know the adherence of this treatment.

Conclusion: Information and Communications Technology could represent an useful tool for allowing a follow-up and applying a specialized rehabilitation treatment for those patients that can't attend regularly to the Burn Unit rehabilitation, due to remoteness or moving difficulties.

O17.03 The effect of manual skin techniques on skin perfusion

U. Van Daele 1 , P. Moortgat², J. Meirte', T. Vanhullebusch', M. Anthonissen², K. Maertens², R. Clijsen $^{3}$

${ }^{1}$ University of Antwerp, Department of Rehabilitation Sciences and Physiotherapy, ANTWERP, Belgium

${ }^{2}$ Oscare, ANTWERP, Belgium

${ }^{3}$ Thim Van der Laan University College Physiotherapy, LANDQUART, Switzerland

Objectives: Prolonged inflammation with increased perfusion increases the number of fibroblasts, endothelial cells and inflammatory mediators leading to scar hypertrophy. Manual and mechanical skin techniques are used to decrease scar hypertrophy. We want to investigate if the applied pressure during manual skin techniques affect skin perfusion.

Methods: An interventional pre-post single arm study is performed in healthy adults. Skin perfusion was measured by means of laser speckle imaging. Two types of manual skin massage techniques, the soft gliding technique and the more intense skinfold technique, were both performed at two intensity levels. The intensity of the manual technique was controlled by real-time feedback.

Results: 23 subjects enrolled into the study. Only the application of the high intensity $\left(2.5-3 \mathrm{~kg} / \mathrm{cm}^{2}\right)$ skinfold technique showed a significant increase in perfusion (mean $+\mathrm{SD}$ perfusion: pre $=65.33 \mathrm{AU} \pm$ 10.87AU; post $=105.11 \mathrm{AU} \pm 35.84 \mathrm{AU}$, paired sample $\mathrm{t}$-test $\mathrm{p}<.000)$.

Conclusion: Soft manual skin techniques used during physical scar management, as gliding techniques, can be performed at higher intensity without increasing skin perfusion. The typical more intense techniques, as skinfold techniques, should be performed at low intensity. These results help to 
determine the intensity level of manual skin techniques without increasing skin perfusion.

Picture 1: https://www.eventure-online.com/parthen-uploads/89/9EBA/add_2_534982_42f73a9ff2f1-4ade-8787-299760e32d43.png

Picture 2: https://www.eventure-online.com/parthen-uploads/89/9EBA/add_534982_42f73a9ff2f1-4ade-8787-299760e32d43.png

Picture 3: https://www.eventure-online.com/parthen-uploads/89/9EBA/add_1_534982_42f73a9ff2f1-4ade-8787-299760e32d43.png

O17.04 The fibroblast under tension in physiotherapeutic mobilization of patients with burn scars

Koller $^{1}$, V Gut ${ }^{2}$

${ }^{1}$ Rehaklinik Bellikon, BELLIKON, Switzerland

${ }^{2}$ University Hospital Zurich, ZÜRICH, Switzerland

Objectives: Wound- healing- adapted dosing in mobilisation of patients with burn scars together with functional stimulation of the scar are prerequisites for physiological scar healing. Manual stimulation of the skin activates the fibroblasts via the physiological process of mechanotransduction. The aim of this study is to provide evidence for the beneficial effects of wound healing adapted dosing in mobilisation of burn patients.

Methods: A literature review on the workingmechanisms underpinning the dose dependency of manual stimulation of the skin in order to enhance scar remodelling together with a pilot study on the effects of manual mobilisation of patients with burn scars.

Results: Clinical considerations on wound healing adapted dosing, combined with mechanotransduction related approaches towards scar remodellingevident tissue physiology, have shown good clinical outcomes in the treatment of burn patients over the last 5 years. Overdosesage due to excessive mechanical mobilisations in the treatment have decreased. The intertester reliability of connective tissue resistances was moderate with ICC2 $=0.67(\mathrm{R} 1)$ and good with ICC2 $=0.80(\mathrm{R} 2)$. The clinical results have improved empirically.

Conclusion: Tissue- responsive and wound healing -adapted physiotherapeutic mobilisation is the correct way towards optimal scar remodelling, which in the longer term leads to functional tissue extension and displacement. It takes time and many therapeutic interventions to obtain beneficial results. Microtraumatic tissue damage caused by overdosed mobilisation should be avoided.

O17.05 Predicting exercise capacity following pediatric burns

M. Akkerman ${ }^{1}$, LJ Mouton², S De Groot ${ }^{3}$, AS Niemeijer ${ }^{4}$, SMHJ Scholten-Jaegers ${ }^{5}$, ME Van Baar ${ }^{6}$, MM Stoop ${ }^{7}$, LHV Van der Woude ${ }^{2}$, MK Nieuwenhuis ${ }^{8}$

${ }^{1}$ Association of Dutch Burn Centres, Burn Centre Groningen, GRONINGEN, Netherlands

${ }^{2}$ University of Groningen, UMCG, Center for Human Movement Sciences, GRONINGEN, Netherlands

${ }^{3}$ Amsterdam Rehabilitation Research Center I Reade, AMSTERDAM, Netherlands

${ }^{4}$ Martini Academy, Martini Hospital, GRONINGEN, Netherlands

${ }^{5}$ Burn Centre Groningen, Martini Hospital, GRONINGEN, Netherlands

${ }^{6}$ Association of Dutch Burn Centres, Burn Centre Maasstad Hospital, ROTTERDAM, Netherlands

${ }^{7}$ Association of Dutch Burn Centres, Burn Centre Red Cross Hospital, BEVERWIJK, Netherlands

${ }^{8}$ Association of Dutch Burn Centres, Burn Centre Groningen, Martini Hospital, GRONINGEN, Netherlands

Objectives: Recovery of exercise capacity is an important prerequisite to regain pre-injury levels of functioning and quality of life. This study aimed to describe exercise capacity in pediatric burn patients during the initial six months after hospital discharge, and examine whether its recovery can be predicted from burn characteristics, sociodemographic characteristics, and/or prior assessment of exercise capacity.

Methods: Eligibility criteria for this prospective cohort study were age 6-18 years, admission to a Dutch burn center with burns covering $\geq 5 \%$ of total body surface area (TBSA), and/or a length of 
stay of $\geq 2$ weeks. Exercise capacity was measured at discharge, and 6 weeks, 3 months, and 6 months after discharge using the Steep Ramp Test (SRT). Outcomes were compared to data from healthy Dutch peers. ${ }^{*}$ Multilevel regression analyses were performed to determine the course of exercise capacity and to identify its predictors.

Results: Twenty-four pediatric patients (15 boys / 9 girls) with burns affecting 0.1-34\% TBSA were included. At group level, exercise capacity was low at discharge and did not reach healthy reference values within six months, despite significant improvement over time. At individual level, the course of exercise capacity varied widely. Six months after discharge, $48 \%$ of participants scored more than one standard deviation below healthy age- and sex-specific reference values. SRT outcomes at six weeks and three months were the best predictors of exercise capacity six months after discharge, explaining respectively $76 \%$ and $93 \%$ of variance.

Conclusion: Half of the participants did not achieve healthy reference values of exercise capacity and was therefore considered 'at risk' for diminished functioning and quality of life. As early assessment of exercise capacity with the SRT seems able to identify those patients, we recommend incorporating the assessment of exercise capacity in pediatric burn patients by the SRT six weeks after discharge. *(Bongers et al., 2013: PMID: 23723384)

O17.06 Fatigue after burn injury - is it a problem?

ASM Enblom

Uppsala University Hospital, UPPSALA, Sweden

Objectives: Physical function and daily activities are often affected after burn injury. Many patients experience complications like pain, muscle weakness, scar contractures, and scar hypertrophy. The burn injury might also lead to fatigue, a symptom that is rarely assessed.

Fatigue, also brain or mental fatigue, is described as a deep, mental exhaustion without apparent cause and in need of long recovery. Fatigue is a well-known sequela after traumatic brain-injury or diseases of the nervous system, such as stroke, multiple sclerosis, or Parkinson's disease. It has also been described among the burn population with negative impact on daily activities and reduced capacity for work.

The aim of this study is to examine if, and to what extent, fatigue is a problem among our burn victims.

Methods: Twenty consecutive patients scheduled for follow-up at the Burn center's outpatient clinic in Uppsala, will be asked to fill out a questionnaire, the Fatigue Severity Scale (FSS), as screening. The FSS was chosen since it is available, valid, and reliable in Swedish and also easy to fill in and administer. The next step will be to examine possible correlations between fatigue, TBSA, return to work and/or quality of life.

Results: If the results show that fatigue is a common problem among our patients a written information will be put together with facts about fatigue, known causes and symptoms, and possible strategies to make everyday life work in a better way.

Conclusion: This is an ongoing quality-work and the results will be presented at the European Burns Association Congress in September 2019.

O17.07 Facilitators and barriers of early mobilization in critically ill patients with burns: a survey

$\underline{\text { Y Dikkema }^{1}}{ }^{\text {, L.J. Mouton }}{ }^{2}$, S.M.H.J. Scholten - Jaegers ${ }^{3}$, C.P. Van der Schans ${ }^{4}$, M.K. Nieuwenhuis ${ }^{1}$

${ }^{1}$ Association of Dutch Burn Centres, Burn Centre Groningen, Martini Hospital, GRONINGEN, Netherlands

${ }^{2}$ Center for Human Movement Sciences, University Medical Center Groningen, GRONINGEN, Netherlands

${ }^{3}$ Burn centre Groningen, Martini hospital, GRONINGEN, Netherlands

${ }^{4}$ Hanze University of Applied Sciences Groningen, Research group Healthy Ageing, GRONINGEN, Netherlands

Objectives: There is growing evidence and recognition of the benefits of early (timely) mobilization of critically ill patients. Timely mobilization may also benefit patients with extensive 
burns and/or inhalation injury, critically ill or not. This does however not mean that it has already been embraced and implemented by all. Timely mobilization of this kind of patients is an intensive, complex process that requires multidisciplinary input and cooperation. To gain insight in the facilitators and barriers for early mobilization in critically ill patients in general, various surveys have been developed. The aim of our study is to find a survey suitable to assess facilitators and barriers in burn patients specifically.

Methods: A systematic review was conducted. The databases PubMed, Cinahl and Web of Science were searched for relevant publications. The search was unrestricted in language and time period. Additionally, references from retrieved publications were checked. Studies were eligible if they concerned: critically ill patients or ICU, facilitators and barriers, early mobilization and a survey or questionnaire. When the search was completed, duplicates were removed. The titles and abstracts of all records identified by the search were independently screened by two researchers (YD, LM) to determine eligibility. When there was lack of clarity about the inclusion of an article, this was resolved by discussion. Data were extracted regarding a.o. study characteristics, clinimetric properties, intended for which professions, theoretical framework. For reporting, the Preferred Reporting Item for Systematic Reviews and Meta-Analysis (PRISMA) was applied.

Results: The search identified 534 studies. After screening title and abstract, 35 articles were eligible. Full text articles are being read to determine inclusion. Data extraction from included articles is underway. Results will be presented.

Conclusion: Having identified a suitable survey, which can be adjusted if required, we will proceed to investigate the facilitators and barriers of mobilization in critically ill burn patients.

O17.08 Tension reducing taping as a mechanotherapy for hypertrophic burn scars: Preliminary results from a pilot study.

T Vanhullebusch ${ }^{1}$, P Moortgat ${ }^{2}$, M Anthonissen ${ }^{3}$, J Meirte ${ }^{1}$, C Lafaire ${ }^{4}$, L De Cuyper ${ }^{4}$, K. Maertens ${ }^{5}$, U Van Daele ${ }^{1}$

${ }^{1}$ Oscare - University of Antwerp, MERKSEM, Belgium

2Oscare, MERKSEM, Belgium

${ }^{3}$ Oscare - KULeuven, MERKSEM, Belgium

${ }^{4}$ Oscare - ZNA Stuivenberg, MERKSEM, Belgium

${ }^{5}$ Oscare - Vrije Universiteit Brussel, MERKSEM, Belgium

Objectives: Hypertrophic scarring and contraction following burn injuries remain a real concern and clinical challenge, causing physical, psychological and social burden. It is generally accepted that hypertrophy and scar contraction can be minimized by reducing mechanical tension. Applying elastic therapeutic tape around the surface of the scar and thus altering mechanical forces on the skin is a newly developed therapy to reduce tension on burn scars. The aim of this pilot study was to determine the scar characteristics that could benefit from this therapy in order to include them in a randomized controlled trial (RCT).

Methods: Patients with hypertrophic scars located between joints, from 3 months to one year after full wound closure were eligible to be enrolled. They were treated with tension reducing taping technique for 3 months and assessed before the first treatment, after one, three, six and 12 months. POSAS was used for subjective assessment. Objective measurements were performed with Cutometer (pliability), Chromameter (colour), Tewameter (TEWL), Corneometer (hydration) and high-frequency ultrasound (dermal thickness). Statistics included descriptives and one-way repeated measures manova. Scar characteristics that showed statistically significant improvement over time with effect sizes higher than 0.8 (cohen's d) were found eligible to include in the RCT.

Results: The first results on 10 patients with a mean scar age of 7.1 months (SD 2.92) are presented. Scar characteristics that met the above mentioned criteria were itch ( $p=.004, d=2.23$ ), colour $(\mathrm{p}<.0005, \mathrm{~d}=1.19)$, pliability $(\mathrm{p}<.0005, \mathrm{~d}=-1.64)$ and dermal thickness $(\mathrm{p}=.001, \mathrm{~d}=1.19)$. Pairwise comparisons presented significant results after 3 months for colour and after 12 months for itch, pliability and dermal thickness. 
Conclusion: Studies which investigate tension reducing therapies to improve scar outcomes should include itch, colour, pliability and dermal thickness as variables. The results for itch are remarkable and call for more research on the correlation between itch and tension.

O17.09 Burn scar contractures that limit daily functioning in children and adolescents: a preliminary longitudinal study

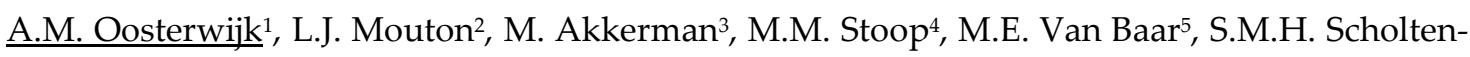
Jaegers ${ }^{3}$, C.P. Van Der Schans ${ }^{1}$, M.K. Nieuwenhuis ${ }^{3}$

${ }^{1}$ Hanze University of Applied Sciences Groningen, GRONINGEN, Nederland

${ }^{2}$ University of Groningen, University Medical Center Groningen, GRONINGEN, Netherlands

${ }^{3}$ Association of Dutch Burn Centres, Burn Centre Groningen, GRONINGEN, Netherlands

${ }^{4}$ Association of Dutch Burn Centres, Burn Centre Beverwijk, BEVERWIJK, Netherlands

${ }^{5}$ Association of Dutch Burn Centres, Burn Centre Rotterdam, ROTTERDAM, Nederland

Objectives: Scar contractures are a well-known sequela of burn injury, that is specifically relevant as it may limit function. Until now, longitudinal studies on scar contractures are scarce and not focussed on function. The present study aims to describe the course of prevalence of scar contractures that limit function in children and adolescents after burns during the initial six months after discharge.

Methods: From a prospective cohort study, range of motion (ROM) data were used of 57 extremity joints measured in 20 paediatric burn patients (6-18 year; mean \pm SD TBSA burned: $12.6 \pm 9.3 \%$ ) at discharge (T0), and six weeks (T1), three months (T2) and six months (T3) after discharge. A scar contracture limiting function was defined as measured ROM lower than the functional ROM necessary to perform daily activities by unimpaired subjects. Prevalences were calculated on group and joint level.

Results: The prevalence of subjects that had one or more scar contractures limiting function was $89.5 \%$ at discharge (T0) and still substantial six months later (T3) (76.5\%). At discharge (T0), more function limiting contractures were found in lower (53.3\%) than upper extremity joints $(29.7 \%)$ (adjusted p-value: .092). Over time (T1-3), lower extremity prevalence fluctuated slightly (26.3\%; $25.5 \%$; $34.6 \%)$ as well as upper extremity prevalence $(29.4 \% ; 21.4 \% ; 30.8 \%)$. Limitations were primarily present in the elbow flexion, knee flexion, and ankle dorsal flexion direction.

Conclusion: A large number of children and adolescents still had one or more scar contractures limiting function six months after discharge. Substantial longitudinal studies, until the end of scar maturation, are needed to increase knowledge on the course of scar contractures limiting function in order to support improvements in burn care.

O17.10 Understanding the experience of adhering to burn scar treatments in children with burns and their caregivers

IKilley ${ }^{1}$, M Simons ${ }^{2}$, R Kimble ${ }^{2}$, Z Tyack ${ }^{1}$

${ }^{1}$ Centre for Children's Burns and Trauma Research, SOUTH BRISBANE, Australia

${ }^{2}$ Queensland Children's Hospital, BRISBANE, Australia

Objectives: Health professionals often recommend that children with burn scars use a range of complex scar treatments, including pressure garments and silicone gels, for months or years after a burn. Currently little is known about the lived experience of adhering to these treatments. The objective of this study was to establish a grounded theory of adhering to scar treatments for children with burns and their caregivers.

Methods: Eligible children and caregivers were recruited from the tertiary burns centre at the Queensland Children's Hospital, Brisbane. Eligible children were aged between 8 and 16 years and requiring scar treatments to manage burn scarring. Caregivers with children aged between 0 and 16 years and using scar treatments were also interviewed. Data were analysed using line-by-line coding, then tentative categories were further developed using theoretical sampling. Other data collected included socio-demographics, caregiver health literacy and comorbidity, and scar symptoms.

Results: Fourteen interviews have currently been completed with caregivers of children using scar treatments $(n=12)$ and children themselves $(n=2)$. Further interviews are in progress. Caregivers had a median age of 35 years (IQR: $31.5-37.5)$ and were predominantly female $(n=11)$. The two children 
were male and female (aged 12 and 8 years). Tentative conceptual categories included using distraction techniques in young children, incorporating scar treatments into established routines, knowing a child's limits and trusting expertise of other caregivers (e.g. day care providers, grandparents etc.).

Conclusion: An emerging grounded theory on the experience of adhering to scar treatments for children with burns and their caregivers will be outlined. This theory will be used to develop an intervention that improves adherence to scar treatments in children with burns and will guide the assessment of children undergoing scar treatments.

O18.01 Comparison of international burn injury data

Y Singer ${ }^{1}$, Y Singer $^{2}$, H Cleland ${ }^{2}$, M Peck², B Gabbe ${ }^{4}$, B Phillips ${ }^{5}$, PQ Bessey ${ }^{6}$

${ }^{1}$ Victorian Adult Burn Service, PRAHRAN, Australia

${ }^{2}$ Victorian Adult Burn Service, The Alfred, MELBOURNE, Australia

${ }^{3}$ Arizona Burn Center, PHOENIX, USA

${ }^{4}$ Monash University, MELBOURNE, Australia

${ }^{5}$ BData, MINNEAPOLIS, USA

${ }^{6}$ William Randolph Hearst Burn Center, NEW YORK CITY, USA

Objectives: Comparing burn injury data on an international basis can offer significant benefits to patient outcomes. As different countries develop burn clinical quality registries, it is possible to compare similarities and differences. The purpose of this study was to compare characteristics of patients treated in burn centres in Australia, New Zealand, and the United States.

Methods: This study included admissions to the BRANZ and the NBR between July 12015 and June 30 2017. Data from each registry were extracted for the study including demographics, burn severity and characteristics, and in-hospital outcomes. Data were categorised according to predefined, harmonised definitions between the two registries and described as counts/percentages or mean/SD.

Results: There were 5,946 admissions to the BRANZ, and 48,012 admissions to the NBR over the study period. Across both registries there were a greater number of males (BRANZ $\mathrm{n}=3,988$ [67.1\%]; NBR $\mathrm{n}=31,609$ [65.9\%]) compared to females (BRANZ $\mathrm{n}=1,958$; NBR $\mathrm{n}=16,369$ ), and adults (BRANZ $\mathrm{n}=3,984$ [67.0\%]; NBR $\mathrm{n}=35,121$ [73.2\%]) compared to children (BRANZ $\mathrm{n}=1,962 ; \mathrm{NBR} \mathrm{n}=12,891$ ). There was no difference between the registries with respect to the proportion of males $(p=0.07)$, but the NBR had a larger proportion of adult admissions compared to BRANZ $(p<0.001)$. Scalds were the most common cause of burn across both registries (BRANZ 37.5\%; NBR 32.7\%).

Conclusion: BRANZ cases had larger burns than NBR cases, whereas NBR cases were older, more frequently had inhalation injuries, and a higher mortality. This study is a small step towards harmonising and comparing burn injury data internationally. This is the first attempt to compare burn data between countries with mature burn registries, and further efforts towards harmonisation are warranted.

\section{O18.02 LAUSANNE MASSIVE BURNS COHORT - 18 YEARS REVISITED}

IN Meuli, M Berger, O Pantet, W Raffoul

Lausanne University Hospital, LAUSANNE, Switzerland

Objectives: Our analysis aimed at determining if the stepwise changes that occurred in the surgical management of very severely burned adult patients (TBSA $\geq 50 \%$ ) between 2000 and 2018 had impacted outcomes in the Lausanne University Hospital Burn Centre Intensive Care Unit (ICU).

Methods: Altogether, 70 adult patients were admitted in our Burn Centre with burns $>50 \%$ TBSA. Twenty-six patients did not benefit from full therapy and were not included (early withdrawal $<48 \mathrm{~h}$ ), leaving 44 for analysis. Patients were stratified in three subgroups (2000-2005; 2006-2011; 2012-2018) determined by changes in the organization of the ICU and in treatment protocols. Following data were recorded: demographic and burn characteristics, critical care treatment received during ICU stay, surgical therapy received and outcome variables (length of stay, length of mechanical ventilation, mortality).

Results: The 44 patients had a median age of 36 years and were burned on $65 \%$ TBSA. Those characteristics remained stable over all three periods, as did almost all other patients' variables. 
Unexpectedly, we did not find any significant differences in outcomes. Median length of mechanical ventilation was 25 days and remained stable. Median length of stay per\% TBSA affected was 0.96 days and showed a tendency towards shortening over time, albeit not significant. Patients were grafted on a median area of $51 \%$ TBSA with a tendency to increase over the last period. Overall mortality was $27.5 \%$ and stable over all three periods.

Conclusion: Despite a general impression of improvement in care over the last 18 years, outcomes show no significant differences between 2000 and 2018. Whether excessive optimism, insufficient documentation or inappropriate choice of outcomes plays the major role remains open to discuss but this result should bring us to look straight into our practices when pursuing our never-ending quest to better care.

O18.03 Reliability of the Norwegian version of the Patient and Observer Scar Assessment Scale

$\underline{\text { M Hjellestad }}{ }^{1}$, LI Strand ${ }^{2}$, A Nesheim¹, R Ljones Brekke1, GE Eide², BE Bassoe Gjelsvik ${ }^{1}$

${ }^{1}$ Haukeland University Hospital, BERGEN, Norway

${ }^{2}$ University of Bergen, BERGEN, Norway

Objectives: The purpose of this study was to examine reliability of the translated Norwegian version of POSAS for use in clinical practice and research.

Methods: The study was carried out during regular outpatient visits at a hospital burns clinic. A cross-sectional design was used to examine inter-tester reliability of the POSAS-NV, and a longitudinal design to examine intratester reliability and validity. Twenty-six adults and 24 children were included following written informed consent. Exclusion criteria: mature scars, severe cognitive dysfunction and too poor Norwegian language skills to understand information and instruction given and to score the Patient Scale. Fifty unique scars were assessed by three observers; nurse, plastic surgeon, physiotherapist (Observer Scale), and by patients or next-of-kin (Patient Scale). The Observers reassessed the scars by digital photos after one week. The patients or next-of-kin re-assessed their scars at home, two days after the first assessment.

Results: At the time of the accident, the mean (standard deviation) age was 4.8 (3.9) years for the children and 35.2 (18.5) years for the adults. The scars were first assessed after mean (SD) 16.9 (46.2) months.

Inter-tester reliability of the Observer Scale parameters varied between intraclass-correlations (ICC 2.1$)$ 0.203-0.728. Only Pliability demonstrated acceptable inter-tester reliability $(>0.70)$ between observers 1:2 and 1:3, and Overall Opinion between Observers 1:3. Intratester reliability for the parameters was moderate to high $\left(\mathrm{ICC}_{2.1}=0.536-0.858\right)$. All parameters demonstrated acceptable intratester reliability (ICC $2.1>0.70)$, apart from Vascularity (0.575) and Pigmentation (0.638) for Observer 1 and Vascularity (0.640) and Surface Area (0.536) for Observer 3. Smallest Detectable Change (SDC) for Overall Opinion was 2.4 (scale 1-10).

The Patient Scale demonstrated high test-retest reliability for all parameters, ICC $2.1 \geq 0.728$. SDC for Overall Opinion was 2.6.

Conclusion: Reliability of POSAS-NV was found satisfactory when the same Observer or the Patient assessed and reassessed the scars.

O18.04 Neuropsychological and behavioural side effects of repetitive sedation in paediatric burn care: alternative solutions

SMHJ Scholten, GIIM Beerthuizen, M.K. Nieuwenhuis

Burn Centre Groningen, GRONINGEN, Netherlands

Objectives: Children up to four years of age are at risk for scalds. Most scalds are second degree and treatment is painful. Dependent on its depth, spontaneous re-epithelialization takes 2 to 3 weeks, requiring multiple wound dressing changes in the meantime. To prevent pain and anxiety, in many burn centres wound dressing changes are done under sedation. The disadvantage, is that children receive multiple anaesthesia in a period when their developing brain is vulnerable. There is a growing concern about the potential neurotoxic effects of anaesthetic and sedative drugs.

Methods: Literature review and case reports. 
Results: From our literature review, it emerged that three large studies have made important contributions to our understanding of the clinical effects of anaesthesia exposure (N-methyl-Daspartate (NMDS)-mediated and ๑-aminobutyric (GABA) mediated) on neurodevelopment in children; the GAS (General Anaesthesia compared to Spinal anaesthesia), PANDA (Paediatric Anaesthesia NeuroDevelopment Assessment), and MASK (Mayo Anaesthesia Safety in Kids) studies. Their overall finding was that single anaesthesia does not influence brain function. However, parents did report problems with mental skills. Children with multiple exposures to anaesthesia had modest declines in fine motor skills and more learning and behavioural problems. The growing evidence that multiple exposures to anaesthesia may have deleterious effects on the developing brain, presents doctors and parents with a difficult dilemma, especially in burn care. They have to consider if a skin transplantation is really necessary in a child and whether wound dressing changes really have to be performed under sedation.

Conclusion: In the Burn Centre Groningen, the Netherlands, wound care in young children has been performed without sedation for many years. Alternative solutions have been developed and are applied, to fit the individual child. All different methods are based on distraction and building confidence. Our various solutions for children to up to 3 years old will be presented.

O18.05 Factors Affecting the Length of Stay of Burn Injury Patients in Two Hospital Burn Unit in Central Jakarta, Indonesia

A. Wardhana, G. Winarno, S. Tanjunga, R. Widyana

Ciptomangunkusumo Hospital, DKI JAKARTA, Indonesia

Objectives: The purpose of this study is to determine some factors in burn injury patients that has a significant contribution towards LOS.

Methods: This is a retrospective cross-sectional study, collected through the medical records across two hospitals burn unit which are Islamic Hospital Cempaka Putih Jakarta and Ciptomangunkusumo Hospital Jakarta. Inclusion criteria is burn patients treated in either one of the burn unit from 2017-July 2018 , while the exclusion criteria is patients who were admitted $>7$ days post burn day, readmission, electrical injury, not operated, death as outcome and incomplete data in medical record.

Results: There are 75 patients which met the inclusion criteria, and we analyzed some variables that includes age, gender, operation timing, cause of burn injury and TBSA. The variables that are statistically significant $(p<0.05)$, are cause of burn injury $(p=0.025)$, where flame with the highest average (22.32 days), and a positive correlation of TBSA $(\mathrm{p}=0.00)$.

Conclusion: Out of 5 variables analyzed, only 2 variables are significant, which are TBSA and etiology. Burn injury caused by flame is more likely to have longer LOS rather than gas explosion and scald, while the higher TBSA is, the more likely the LOS is longer.

Picture 1: https://www.eventure-online.com/parthen-uploads/89/9EBA/add_1_541112_f080f9863464-463d-ae67-95bb71488d46.png

Picture 2: https://www.eventure-online.com/parthen-uploads/89/9EBA/add_541112_f080f9863464-463d-ae67-95bb71488d46.png

O19.01 Identifying factors associated with toxic shock syndrome in paediatric burns patients

$\underline{\text { AV Shaw }}{ }^{1}$, M Marks ${ }^{2}$, J Ross ${ }^{1}$, I Jones ${ }^{1}$, A Kearns ${ }^{3}$, LSP Moore ${ }^{1}$, D Collins ${ }^{1}$

${ }^{1}$ Chelsea \& Westminster Hospital, LONDON, United Kingdom

2Brighton and Sussex Medical School, BRIGHTON, United Kingdom

${ }^{3}$ Public Health England, LONDON, United Kingdom

Objectives: Limited research exists regarding toxic shock syndrome (TSS) as a rare but potentially fatal complication of paediatric burns. To further characterise this, a retrospective observational study was undertaken at the London regional burns unit.

Methods: Clinical and laboratory data was collected for paediatric burns patients with clinically suspected TSS treated between May 2016-2018. Patients were categorised according to final clinical diagnosis; TSS with confirmed Staphylococcus aureus or Streptococcus pyogenes, and non-TSS febrile illness. 
Results: 59 patients were initially treated as TSS; mean presentation age was 21.8 months. 40 retained a TSS diagnosis with 19 receiving an alternate diagnosis. Period prevalence was $10.2 \%$ of admitted paediatric burns and incidence of 51/1000 paediatric burn patients/year.

In the TSS group, mean symptom onset was 3.9 days with 20/40 presenting with diarrhoea or vomiting and 15/40 with a rash. 38/40 subsequently grew $S$. aureus from wound cultures (16/19 tested demonstrated exotoxins TSST-1 or SEA-SEC) and 1 S. pyogenes.

The mean antimicrobial duration for TSS patients was 9.3 days. Six received FFP, seven required PHDU and one ICU. 30-day mortality was $0 \%$.

There was no significant difference between groups regarding the burn size or depth, date of symptom onset, presence of rash or gastrointestinal symptoms or presentation PEWS.

Patients who retained a TSS diagnosis took longer to defervesce below $38^{\circ} \mathrm{C}$ (mean 18.3 vs 13.3 hours) and also lived in less deprived areas (mean English indices deprivation score 14545 vs 10451).

Conclusion: This is the largest study, to date, of paediatric burn-related TSS. Our data shows a higher prevalence than previously noted. Increased vigilance of patients presenting with gastrointestinal symptoms or rash post burn, should heighten suspicion of possible TSS. If suspected, appropriate molecular microbiological investigation and aggressive antimicrobials should be initiated. Following this study, we have introduced a clinical guideline for possible paediatric post-burn TSS in our unit.

Picture 1: https://www.eventure-online.com/parthen-uploads/89/9EBA/add_2_540818_d38203f225ac-4b6b-9743-9cdd65f954b6.png

Picture 2: https://www.eventure-online.com/parthen-uploads/89/9EBA/add_540818_d38203f225ac-4b6b-9743-9cdd65f954b6.png

Picture 3: https://www.eventure-online.com/parthen-uploads/89/9EBA/add_1_540818_d38203f225ac-4b6b-9743-9cdd65f954b6.png

Picture 4: https://www.eventure-online.com/parthen-uploads/89/9EBA/add_3_540818_d38203f225ac-4b6b-9743-9cdd65f954b6.png

O19.02 Carbapenemase producing gram negative bacilli in a Burn Care Center in Tunisia

MA Mokline, DL Debbiche, BH Ben Ali, EK El Faleh, HF Fraj, BM Ben Saad, EI El Jami, TL Thabet, AAM Messadi

Trauma and Burn Center, TUNIS, Tunisia

Objectives: The purpose of this study was to evaluate the portage of carbapenemase in burn patients in tunisia and to identify risk factors.

Methods: A prospective, monocentric study was conducted from May to September 2018 in a burn unit in Tunisia. In all patients, a search for BMR carriage (skin, rectal, bronchial, urinary, and catheter samples) and for carbapenemases (VIM, NDM and OXA 48) by polymerase chain reaction (PCR) were carried out initially at admission then weekly.

Results: During the study period, 31 patients were included. The mean age was 35 years. They were 17 men and 14 women. The average burned surface area was 39\%. Patients were transferred from another hospital structure in $80 \%$ of cases with a delay of 36 hours. Isolated MRB were: Enterobacteriaceae producing extended-spectrum Blactamases (ESBLs) (49\%), Carbapenemaseproducing Enterobacteriaceae (CPE) (87\%), Methicillin- Resistant Staphylococcus aureus Strains (19\%) and glycopeptide-resistant enterococci (GRE) (13\%). Carbapenemase encoding genes were detected in 22 patients: 12 at admission and 10 at the first week post admission. The genes detected were New Delhi metallo- $\beta$-lactamase (92\%), Verona integron-encoded metallo- $\beta$-lactamase $(58 \%)$, and oxacillinase- 48 (58\%). Univariate analysis identified risk factors associated with acquiring CPE were: secondary transfert; indwelling device, antibiotic use of aminosids and amox-clavulanic acid and non-compliance with hygiene measures.

Conclusion: Detection of Carbapenemase-producing Enterobacteriaceae (CPE) in our study was higher $(87 \%)$, essentially due to secondary transfer from endemic regions of CPE. So, detection and isolation of these patients and strengthen infection control measures allows us to improve therapeutic efficacy and improves their prognosis. 
O19.03 Managing an outbreak of a Multi-Drug Resistant Acinetobacter baumannii. Lessons learnt.

T K Randawa, J Stallard, PM Muthayya, A Phipps, U Anwar

Pinderfields Regional Burns centre, WAKEFIELD, United Kingdom

Objectives: Antimicrobial resistant organisms are on the rise. The World Health Organisation have published a list of priority pathogens to focus research and development on; many of which are commonly seen in patients with burns. It is inevitable that burns centres will have to manage these organisms more frequently. We aim to present our recent lessons learnt from an outbreak of Acinetobacter baumannii, carbepenem resistant (ABCR).

Methods: Multidrug resistant organisms are on the rise.

We had a numerous discussion with our infection control department and public health England due to the lack of robust evidence in the literature to develop a stringent management plan. We contained the outbreak with Isolation, Barrier techniques, Environmental management, Theatre management and outpatient isolation.

Burns services are going to be increasingly faced with problematic bacteria. Too our knowledge two other services within the UK have had difficulty with multidrug resistant bacteria.

Results: 1/11/18 Adult Burn Centre closed for admissions due to a confirmed outbreak of ABCR. The unit was closed for admissions for a short period until a management plan could be implemented. At the time of closure, three of the five inpatients had ABCR (no further in patient contracted ABCR). In total five patients were colonised by the same ABCR, of which one patient suffered graft loss requiring debridement and subsequently further grafting.

Following declaration of the outbreak and implementing our management plan no new patients contracted ABCR.

Conclusion: The management of multidrug resistant pathogens is going to be an evolving challenge in the future. We were able to contain the outbreak with stringent department measures and commitment from all the staff involved. We would encourage all units to have a protocol in place to allow for early detection and swift intervention and hope to facilitate discussion on developing an international protocol.

O19.04 Pharmacokinetics of Meropenem: prolonged infusion can be more effective?

A. Pensa ${ }^{1}$, S. Corcione ${ }^{2}$, R. Corgiat Loia², F.V. Segala², A. De Nicolò ${ }^{3}$, G. Fatiguso ${ }^{3}$, MR. Romeo ${ }^{4}$, A. D'Avolio $^{3}$, M. Stella ${ }^{4}$, G. Giovanni ${ }^{2}$, FG Derosa ${ }^{2}$

${ }^{1}$ Burn Centre, CTO Hospital, A.O.U.Città della Salute e della Scienza, TORINO, Italy

${ }^{2}$ Department of Medical Sciences, Infectious Disease,University of Turin, TURIN, Italy

${ }^{3}$ Laboratory of Clinical Pharmacology and Pharmacogenetics, Amedeo di Savoia, TURIN, Italy

${ }^{4}$ Burn Centre, CTO, Turin, Italy, TORINO, Italy

Objectives: To describe the pharmacokinetic (PK) parameters of meropenem in a population of burn patients.

Methods: The study was conducted from January 2016 until April 2018 in the Burn Centre of Turin. Blood samples were collected immediately before (T0), after two hours (T1), and five hours (T2) intravenous drug administration of meropenem.

Plasma meropenem concentrations were determined using an ultra-perfomance liquid cromatography-photodiode array method.

Results: This study enrolled seventeen burn patients with a median age of 57 years with a median TBSA of $34 \%$, In $76 \%$ of population the microbiological blood or wound samples reported Pseudomonas aeruginosa and Acinetobacter baumanni.

The mean Cmax, Cmin, AUC 0-24, half-life (T1/T2), Clereance (CL) and volume distribution (Vd) were: $28,9 \mathrm{mg} / \mathrm{L}, 3.7 \mathrm{mg} / \mathrm{L}, 109,2 \mathrm{mg} / \mathrm{Lh}, 2,4 \mathrm{~h}, 17,11 \mathrm{~L} / \mathrm{h}, 47,6 \mathrm{~L}$ respectively. Six patients achieved a $\mathrm{Cmin} \geq 3,3 \mathrm{mg} / \mathrm{L}$ and seven patients achieved a $\mathrm{Cmax} \geq 28,4 \mathrm{mg} / \mathrm{L}$; while nine patients achieved AUC 0$24 \mathrm{of} 226 \mathrm{mg} / \mathrm{Lh}$. According to a MIC of $0,5 \mathrm{mg} / \mathrm{L}$, all patients satisfied the target AUC/MIC of 125>, but when the MIC rose up to $2 \mathrm{mg} / \mathrm{L}$ (ECOFF), only five patients reached the target AUC/MIC.

Conclusion: The meropenem clereance and distribution is peculiar in burn patients. These data suggest that a prolonged infusion ( 2 hours vs 30 minutes) of meropenem can be more effective in terms 
of PK parameters. In conclusion in these patients it is useful the prolonged administration of meropenem.

O19.05 Epidemiology and risk factors for infection in Burn Centre of Turin. Stella ${ }^{4}$

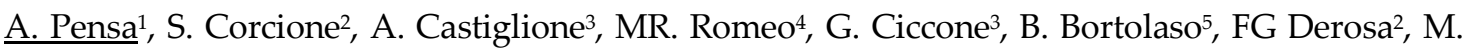

Burn Centre, CTO Hospital, A.O.U. Città della Salute e della Scienza, TORINO, Italy

2Department of Medical Sciences, Infectious Disease,University of Turin, TURIN, Italy

${ }^{3}$ Unit of Clinical Epidemiology, Città della Salute e della Scienza, CPO Piemonte, TURIN, Italy

${ }^{4}$ Burn Centre, CTO, Turin, Italy, TORINO, Italy

${ }^{5}$ Laboratory of Clinical Biochemistry, CTO, A.O.U Città della Salute e della Scienza, TURIN, Italy

Objectives: To determine the demographics and clinical profile of burn patients and to identify their risk factors of infection.

Methods: This is a single center retrospective study conducted from January 2015 and September 2017 which enrolled all burn patients admitted in the Burn Centre.For each patient demographics, clinical,burn,surgical and microbiological data were collected.Revised Baux score (RBS) was calculated for each patient $(\mathrm{RBS}=$ age $+\%$ surface extension of burn wound +17 points in case of mechanical ventilation).

Results: Among the 200 enrolled patients the mean age was 56.6, the male gender was predominat and flame in a domestic setting was the most common circumstance.104 patients were affected by at least one comorbiliy being cardiovascular (34\%) and psychiatric disease (13\%) the most frequent.

During the ricovery 128 patients needed a surgery procedure, 108 patients had a contral venous catheter, 68 patients were supported by mechanical ventilation and 27 patients underwent to dialysis for acute kidney insufficiency. According to ABA criteria the population was divided in two groups (non-infected and infected), the most common microbiological isolates were Acinetobacter baumanni (67\%), Pseudomonas aeruginosa (63\%) Methicillin-resistant Staphylococcus aureus (56\%). The overall in hospital mortality was $12 \%$ but it rose up to $19 \%$ in infected group ( $7 \%$ in non-infected group). The cumulative incidence of infection and the cumulative incidence of death increased by the lenght of hospital days. The risk of infection was superior in patients with invasive devices and with a higher level of RBS.

Conclusion: The mortality in burn patients is strictly associated to infection. As we expected the major risk factors for infection were invasive devices, moreover our data suggest also a possible association between RBS and infection.

O19.06 A Systematic Review of Skin-sparing Debridement in Necrotizing Soft-Tissue Infections

ISuijker, F. Nasroe, A. Pijpe, A. De Vries

Red Cross Hospital, BEVERWIJK, Nederland

Objectives: The surgical technique is likely of influence on the wound size in patients with a Necrotizing Soft-Tissue Infection (NSTI). The use of a skin-sparing technique is expected to give less extensive wounds, earlier healing and less scar-related functional problems, without increasing mortality. Aim of this study was to provide an overview of the current literature on this topic and to describe the indications for and outcomes of the skin-sparing technique in patients with a NSTI.

Methods: A systematic review of the literature was performed according to the PRISMA statement. Two databases (Pubmed and Embase) were searched for known synonyms for 'NSTI' in combination with synonyms for 'skin-sparing'. Selection was done by two independent researchers. Conflicts were discussed with the third, senior author.

First, titles and abstracts were screened. All publications in which a skin-sparing technique was described were selected for full text assessment. Second full text assessment was performed. All articles describing patients treated with a skin-sparing technique were included. Excluded were articles in a language other than the English or Dutch language.

Results: The search resulted in 532 abstracts of which 23 were eligible for full text screening. Fourteen were published in or after 2010, and 11 were published in or after 2015. After full text 
screening, 11 articles were excluded due to: foreign language (6), no patients (3), poster abstract (1). Cross-reference check resulted in one adjacent relevant article. Data are currently being extracted from the 13 included articles. Results will be presented at the conference.

Conclusion: It is evident that there is increased awareness for the skin-sparing technique based on the quantity of publications in the last five years. Definitive conclusions will follow after data extraction.

\section{O20.01 MASS CASUALTIES WITH PEDIATRIC BURN VICTIMS}

\section{DM Enescu, S Stoicescu, D Ionita, I Nacea, R Tatar}

'Grigore Alexandrescu' Hospital, BUCHAREST, Romania

Objectives: Burns are often encountered in mass casualties and increase the severity.

An unusual and extremely grave accident that happened as a fire broke in a neonates department. Out of 11 victims, 8 infants with burns between $40 \%$ and $80 \%$ TBSA were admitted in our burn center. Despite having treated various burns in neonates, the mentioned accident presented unique aspects.

Another severe accident recently happened as a fire broke out in a nightclub. 8 victims were admitted in our hospital, 6 of them having burns.

Our goals were to ensure the best outcomes.

Methods: Victims of two collective accidents were admitted to our hospital.

8 victims of a fire from a neonate department, infants with burns between $40 \%$ and $80 \%$ TBSA were admitted in our burn center.

A collective accident caused by fire in a nightclub was another extremely difficult situation with which our clinic was confronted. 6 patients were admitted having burns and 2 for intoxication

Results: Response to a neonatal pediatric disaster was good. The collective accident baby victims reached our clinic in a short time and everybody mobilized to cope with the situation. Five of the eight victims survived, returned to their families and had normal subsequent growth. The healing pattern was unique, resembling fetal wound healing.

Some of the victims from the nightclub fire are still admitted but their evolution up to date is favorable.

Conclusion: Collective accidents are overwhelming tragedies.

Neonatal burns pose various extreme risks but ultimately good results are to be obtained even in serious cases. Serious burns in this age group are extremely rare, and this case series shows up a unique experience.

Particular aspects of collective accidents were the large number, higher surfaces affected and good results after adequate treatment.

O20.02 Enzymatic burn debridement in a major burns incident

JR Kiely, IIbrahim, GH Lye, J Stallard, T Pinnock, S Jivan, O Austin, P Muthayya, P Clarke, B Sloan, R Sandhu, MU Anwar

Mid Yorkshire NHS Hospitals, WAKEFIELD, United Kingdom

Objectives: NexoBrid ${ }^{\mathrm{TM}}$ (MediWound Ltd., Israel) is a topical treatment used for enzymatic debridement of burns. Although initially used for small burns, we have gained confidence in using it for burns $>15 \%$ TBSA. In 2019 a household explosion resulted in burns to five patients. We present our experience in using NexoBrid ${ }^{\mathrm{TM}}$ in their management.

Methods: A retrospective study was performed of the patient notes, with review of injury pattern and management. Photography was used with consent.

Results: Five adult male patients were admitted, mean age 37.4 years. Patient 1 had $24 \%$ burns, including circumferential intermediate-deep dermal injury to both arms, hands and fingers. Patient 2 had $24 \%$ burns, with scattered superficial dermal burns. Patient 3 had $17 \%$ burns, including circumferential intermediate-deep dermal burns to both forearms, hands, fingers and ankles. Patient 4 had $13.5 \%$ burns, including circumferential intermediate dermal burns to both wrists. Patient 5 had $5 \%$ burns to his face and hands.

Patients 3-5 were managed on a single theatre list, using NexoBrid ${ }^{\mathrm{TM}}$ to debride their burns at 19 , 16 and 23 hours post-injury. Patient 1 had NexoBrid ${ }^{\mathrm{TM}}$ debridement of his injuries on ITU at 18 hours. 
Patient 2 was treated with dressings. Patients with $>15 \%$ TBSA were treated in an ITU setting with resuscitation fluids using the Parkland formula. All had facial burns, with 1-3 requiring intubation.

None of the patients required escharotomy, despite long multi-level circumferential limb burns. Pain management for debridement involved multimodal analgesia, with patients 4 and 5 remaining awake.

Conclusion: Our department was faced with a major incident resulting in multiple patients requiring burn wound debridement, with the potential of overwhelming the service. Through the use of NexoBrid ${ }^{\mathrm{TM}}$, we were able to perform burn debridement and avoid escharotomy using a single theatre and one ITU side-room. We would recommend its use in similar incidents.

\section{O20.03 ORGANIZATION OF MEDICAL TRANSPORTATION OF 256 PATIENT IN MASS BURN CACUALITY INCIDENT}

$\underline{\text { S Shapovalov }}{ }^{1}$, A. Alekseev ${ }^{2}, K_{\text {Krilov }}^{3}$

${ }^{1}$ All-Russian Center for Emergency and Radiation Medicine by A.M. Nikiforova EMERC, ST. PETERSBURG, Russia

${ }^{2}$ A.V.Vishnevsky National Medical Research Center of Surgery,, MOSCOW, Russia

3SAINT-PETERSBURG I.I. DZHANELIDZE RESEARCH INSTITUTE OF EMERGENCY MEDICINE, ST. PETERSBURG, Russia

Objectives: Optimization of medical transportation of patients in mass burn casuality incident.

Methods: For the analysis of 256 patients with burn injuries of varying severity who were injured in an emergency in Perm (2009).

Results: During the period from 5/12 to 6/12/2009, the evacuation team was four flights MoscowPerm-Moscow (St. Petersburg), during which 47 patients were evacuated. Three flights were on the route Perm - Moscow, one - on the route Perm - St. Petersburg. The duration of the flight ranged from $3 \mathrm{~h} 15 \mathrm{~min}$ (to Moscow) to $4 \mathrm{~h} 15 \mathrm{~min}$ (to St. Petersburg). The total time of operation of the combined evacuation team was 33 hours. During transportation, medical modules were used, which allowed to place on board an IL-76 aircraft heavily burned and conduct intensive therapy, dynamic observation and care, as well as, if necessary, resuscitation.

Conclusion: The basic principles of air evacuation (AE) are formulated:

AE should be able to be implemented on most transport aircraft, ideally any type;

The customer of aviation equipment must provide the conditions under which the design and construction of transport aircraft take into account the possibility of AE;

It is necessary to develop and produce a light, compact and autonomous device for mounting on aircraft platforms of any type suitable for AE.

For timely and well organized evacuation of burned, it is necessary to follow the transportation protocol. The protocol provides for a series of successive stages to establish the feasibility, indications and timing of the evacuation of the victim to a specialized burn center.

O20.04 Mass burn casualty incident of Mati Greece. The experience and statistics of admitting the $80 \%$ of the patients in Plastic Surgery Department of Evaggelismos(PSD).

A Kyriakopoulos, M. Kotrotsiou, G Fiolotakis, I. Manolakis, M. Petroulia, M. Divritsioti, A. Michas, G. Harkiolakis

Evaggelismos Hospital of Athens, ATHENS, Greece

Objectives: The biggest wildfire of Greek history and the way the PSD handled the patients is the main goal of this presentation.

Methods: Review of the literature of mass burn casualty incidents in Greece, review of the archives of the PSDE and personal experience of the authors are used to extract the results. In the modern history of Greece three main mass burn casualty incidents exists, the Hilia wildfire, the Petrola distilling industry incident and the wildfire at Mati which was the biggest of them.

Results: 120 patients were admitted to the hospitals of Athens, 50 with burns and 70 with inhalation problems. 75 came to Evaggelismos hospital 30 of them only with inhalation problems and 45 of them with burns to the PSDE. Out of the 45 burned patients 10 of them were send home with 
minimal superficial or partial thickness burns less than 5\%TBS. 6 of them were intubated and had severe total thickens circumferential burns from $58 \%$ to $95 \% \mathrm{TBS}$ with inhalation injury and were hospitalised in the intensive care unit(ICU), with the everyday presence of one plastic surgery consultant specialised in the Burn Unit, for the first 15 days of hospitalisation. 24 patients were hospitalised in the Plastic Surgery Department(PSD) with 5 of them with need of parkland fluid resuscitation. 6 patients were send to another hospital with Plastic Surgery Burn Unit. Out of the 29 patients with severe burns 6 died and 25 survived. The average TBS was $24.2 \%$ and the mean number of surgeries for each patient was 3 .

Conclusion: Mass burn casualty incidents can be handled with systemic team work according to guidelines.

\section{O21.01 LIMB RECONSTRUCTION IN BURN PATIENTS - A TRUE CHALLENGE}

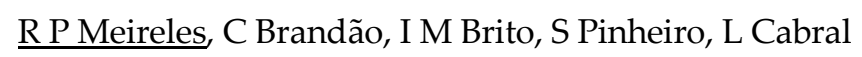

Centro Hospitalar e Universitário de Coimbra, COIMBRA, Portugal

Objectives: Primary burn reconstruction faces a number of additional challenges in the acute setting. The authors undertook a retrospective analysis in order to evaluate the indications, complications and outcomes of tissue transfer, skin substitutes and negative pressure therapy in primary burn limb reconstruction.

Methods: A retrospective analysis of the clinical records of all patients admitted to our Burn Unit and submitted to limb reconstruction in the acute phase, during the last 3 was performed.

Results: Fifteen patients required limb reconstruction with more than skin grafts. Ten required flap reconstruction, with two microsurgical flaps for acute soft tissue reconstruction. In two patients, we used temporary dermal matrix $\left(\right.$ Integra $^{\mathrm{R}}$ ) and in the other three, we used negative pressure therapy. The primary indication for flap reconstruction was tissue deficits with exposure of tendons, nerves, vessels, bone and joint after debridement and it was more often performed in the early period after the injury. The free flap used was the Latissimus dorsi flap. Regional perforator flaps were selected regarding the defect area. Major complications included one total and one partial flap failure.

Temporary dermal matrix was used in two upper limb reconstructions to cover areas of tendon or bone exposure or to optimize healing of functional important structures, such as joints. It was necessary to release scar contractures in a later stage, for both patients.

Pressure negative therapy was used to reconstruct small areas of exposed tendon, with good results and no complications.

Conclusion: Limb reconstruction remains a challenge in the burn patient. Several treatment options are available to optimize burn care and functionality. Free and local flaps have a valuable role in primary burn reconstruction, despite de apparent higher complication rate and they may reduce the total number of surgeries to achieve wound closure.

O21.02 The ALT free flap, a powerful tool for acute burn reconstruction. The Vall d'Hebron University Hospital experience

E. Verdaguer Olivella, J Aguilera Sáez, J. M. Collado Delfa, J Serracanta Domènech, A Monte Soldado, J.P. Barret Nerín

Vall d'Hebron University Hospital, BARCELONA, Spain

Objectives: To show the experience of the Vall d'Hebron University Hospital Burn Center with the ALT free flap for the reconstruction of acute burns.

Methods: Review of retrospective data with descriptive analysis of all the cases of free tissue transfer for acute burns reconstruction in the last 15 years in our Unit.

Results: We performed 29 microsurgical interventions in acutely burned patients between 2005 and 2019. The ALT free flap was the most frequently used microvascular flap for soft tissue coverage, becoming $59 \%$ of the overall microsurgical reconstructions performed in acute burns (17 flaps, 16 patients), followed by Latissimus Dorsi and SCIA free flaps.

$94 \%$ of the patients were male with an average age of 37 years old (ranging from 24 to 71 years old). At least $50 \%$ of the patients were smokers and one had cardiovascular risk factors. Burn etiologies were diverse, including high voltage electric burns in 7 patients. 
The timing between burn episode and microsurgery ranged from 6 to 60 days, with an average of 25 days. The main recipient site was located in the lower extremities (14 cases out of 17), including one Achilles reconstruction with vascularized fascia lata. An angio-TAC was performed in all cases to assess the vascularization of the limb.

Flap survival was complete in 16 cases (94\%). One patient required a skin graft to cover a defect at the proximal third for partial flap necrosis. No complications of the donor area were reported in any case.

Conclusion: According to our experience, the ALT free flap is a versatile and reliable technique in the treatment of acutely burned patients. With adequate preparation and proper indication, we have accomplished high rate survival with no complete flap loss.

\section{O21.03 PEDICLED PERFORATOR FLAPS RECONSTRUCTION IN HIGH-VOLTAGE ELECTRICAL INJURIES}

D Yankov, Y Zayakova, N Nenkova, M Basheva

Military Medical Academy - Bulgaria, Multiprofile Hospital for Active Treatment, VARNA, Bulgaria

Objectives: High-voltage electrical injuries cause severe functional disorders and other burnrelated complications. In this study the authors present their experience with pedicled perforator flaps, designed in accordance with the common patient status, localization and characteristics of the wounds.

Methods: In the period between October 2012 and December 2018, 17 patients with deep highvoltage electrical injuries were managed with pedicled perforator flaps in our unit. For the permanent closure of exposed bones, tendons, joints or nerves a total of 24 flaps were used.

Results: The overall complication rate was $16.7 \%$. The most common complications were partial flap loss (12,5\%) and venous congestion (4,2\%).

Conclusion: Pedicled perforator flaps appear to be a reliable and safe reconstructive procedure for the coverage of soft tissue defects as a result of high-voltage electrical injuries.

O21.04 The use of the biodegradable polyurethane dermal substitute, Biodegradable Temporising Matrix (BTM) in the reconstruction of necrotising fasciitis defects

\section{MID Wagstaff, L Damkat-Thomas, Y Caplash, JE Greenwood}

Royal Adelaide Hospital, ADELAIDE, Australia

Objectives: There has been a five-fold increase in incidence reported of the life-threatening soft tissue infection, necrotising fasciitis. Following radical debridement and wound control, these cases are frequently managed on burns units as they require complex reconstructions. When skin grafts are used, their contraction can result in contracture of joints, or the neck. On the other hand, reconstruction over exposed bone or vessels using tissue flaps can be bulky, with poor contour definition. Healing of both acute reconstructions, and their donor sites, can be challenging in the physiologically unstable patient. We present our experience of use of BTM as a temporising dermal substitute in seven cases.

Methods: All cases in which BTM has been employed in necrotising fasciitis wound reconstruction have been included. Medical records and operation notes were reviewed retrospectively, including clinical photographs. Photographs were taken of all wounds at each surgery and dressing change. The data collected included demographics, length of stay in hospital, description of defect following serial debridement, indication for BTM, time from implantation to split skin grafting and complications.

Results: The cohort includes anterior neck defects exposing major vessels (2/7), multiple exposed ribs on the chest wall (2/7), lower limbs crossing knee or ankle joints (3/7) and a lower limb amputation by hip disarticulation (1/7). The mean age was 58 years ( 31 to 88 years), and the male to female ratio $=$ 3:4. The median inpatient bed days from first debridement to discharge following split skin graft take was 62 days. No BTM was lost and no graft was lost over integrated BTM.

Conclusion: Our data supports previous published evidence that temporising with a dermal substitute is a viable reconstructive option in necrotising fasciitis defects. This is the first series of the use of a completely synthetic dermal substitute in necrotising fasciitis wounds as an effective alternative. 
O21.05 The use of a biodegradable polyurethane dermal substitute BTM (Biodegradable Temporising Matrix) in significant acute burns; outcomes and lessons learned from the first twenty patients.

MID Wagstaff, L Damkat-Thomas, B Schmitt, K Heath, R Kurmis, JE Greenwood

Royal Adelaide Hospital, ADELAIDE, Australia

Objectives: Since its introduction in 2014 we have been using BTM in the management of significant burn injuries. It is a totally synthetic bi-layer, dermal replacement scaffold, composed of a $2 \mathrm{~mm}$ thick, biodegradable polyurethane foam and a non-biodegradable polyurethane film (seal). We aim to present our outcomes and experiences from the first twenty significant burn cases using BTM, along with the evolution of our practice since its adoption.

Methods: Patients were identified via our departmental database. Medical records were retrospectively reviewed, with patient demographics and clinical outcomes being collected, including:

- complications

- number of operative procedures

- time to grafting

- time to range of movement (ROM) and mobilisation

- scar management requirements

- time to commencement of scar management

- scar outcomes

- reconstructive surgical requirements

Results: $75 \%$ of patients were male $(n=15)$ with an age range of $17-94$ years, representing total body surface area burn range of 5-75\%. Mean time from admission to completion of BTM application was 5 days $( \pm S D=7)$ for upper limb sites and 10 days $( \pm S D=11)$ for lower limb sites. Mean time from admission to completion of grafting to BTM sites was 46 days $( \pm S D=6)$. Graft loss over integrated BTM was minimal. Complication rates including BTM loss and graft loss will be presented. Reasons for BTM loss include seroma/heamatoma, infection/contamination, early loss of seal due to shear, and failure to integrate due to fat necrosis beneath the dermal substitute. With experience in the use of BTM, the number of operative procedures for dressing changes has decreased over time and functional and scar outcomes remain promising.

Conclusion: Increasing experience of BTM as a dermal substitute has streamlined our clinical resource utilisation in addition to providing favourable patient outcomes.

O22.01 Establishing a psychological support structure for mass casualty - burn disasters

$\underline{\text { A. Pittermann }}{ }^{1}$, A Fochtmann-Frana ${ }^{2}$, J Nedomansky², B. Schäfer ${ }^{2}$, T. Rath², C. Radtke ${ }^{2}$

${ }^{1}$ General Hospital of Vienna, VIENNA, Austria

${ }^{2}$ Medical University of Vienna, VIENNA, Austria

Objectives: Mass casualty events and especially burn disasters pose an enormous challenge for hospitals and hospital staff. In the recent years, hospital mass casualty response exercises often put particular emphasis on scenarios with a high number of burn victims (terrorist attacks, explosions, burns). As psychological support for victims of mass casualty events and their relatives is part of the hospital alarm plan of the General Hospital of Vienna, Austria, it is essential to establish a psychological support structure for burn disasters as well.

Methods: Based on a retrospective analysis of several hospital exercises in the years 2010-2017 a psychological deployment plan was drafted, including alerting, preparation, documentation, communication within the team and evaluation afterwards. The quantity of the necessary means of staff for the preparation and execution of such a support structure as well as the cornerstones of the psychological emergency support were outlined. The deployment plan was then evaluated during a large hospital exercise in 2018 with the scenario of an explosion at a local festival.

Results: The psychological deployment plan worked well in a large hospital exercise with the scenario of an explosion. To keep track of the patients with the highest demand of psychological support ("triage") as well as the documentation and communication within the psychological emergency team turned out to be the key points during the exercise. 
Conclusion: The provision of psychological support for a short-period of time in an emergency setting can be well-prepared by using a specialized hospital alarm plan. Particular focus has to be put on the "psychological triage" and the documentation of the psychological support. However, the medium- to long-term psychological support requests more and different resources.

O22.02 Health related quality of life 5-7 years after minor and severe burn injuries: a multicentre cross-sectional study

N Trommel $^{1}$, I Spronk ${ }^{2}$, S Polinder ${ }^{2}$, N.E.E. Loey ${ }^{3}$, C.H. Vlies ${ }^{1}$, A Pijpe ${ }^{4}$ J. H. Haagsma², M.E. Baar, $\operatorname{van}^{5}$

${ }^{1}$ Maasstad Hospital, ROTTERDAM, Netherlands

${ }^{2}$ Erasmus MC, ROTTERDAM, Nederland

${ }^{3}$ Association of Dutch Burn Centres, BEVERWIJK, Nederland

${ }^{4}$ Red Cross Hospital, BEVERWIJK, Nederland

${ }^{5}$ Association of Dutch Burn Centres, Maasstad Hospital, ROTTERDAM, Nederland

Objectives: Burn injury can affect health-related quality of life (HRQL). Knowledge concerning long-term HRQL in burn patients is limited. The aim of the study was to evaluate long-term HRQL and to study predictors of impaired long-term HRQL.

Methods: All adults with a length of stay (LOS) of $\geq 1$ day (2011-2012) were invited. Also, adults with severe burns, i.e. $>20 \%$ total body surface area (TBSA) burned or TBSA full thickness $>5 \%$ (20102013) were invited. Participants completed the EuroQol(EQ)-5D-5L+C and visual analogue scale (EQVAS) 5-7 years after burns.

Results: Our study included 256 patients (mean\%TBSA 10\%); 187 patients with minor burns and 69 patients with severe burns. Mean EQ-5D summary was 0.90 and EQ-VAS 83.2 in the minor burn patients, and 0.79 and 78.1 in the severe burn patients. Some problems in at least one dimension were experienced by $81 \%$ of patients with severe burns and $45 \%$ of those with minor burns. However, a minority reported severe or extreme problems; $15 \%$ of those with severe burns and $6 \%$ of those with minor burns. Patients with severe burns reported significantly more problems, except for anxiety/depression. In both patient groups most problems were reported on pain/discomfort. Length of hospital stay, gender and age were associated with lower long-term HRQL (EQ-VAS) in multivariate analyses, whereas only length of stay was associated with a lower summary score.

Conclusion: The majority of patients experienced some problems with HRQL 5-7 years post burn. This emphasizes that burns can have a negative impact on an individual's HRQL, particularly in more severely burned patients, that persists for years. The HRQL dimensions most frequently affected include pain/discomfort and anxiety/depression. Patients with a prolonged hospital stay, females and older patients are at higher risk of poor HRQL in the long-term.

O22.03 Virtual Reality and how to use it effectively in burn care

S.J. Blokzij ${ }^{1}$, K.F. Lamberts ${ }^{1}$, M.K. Nieuwenhuis' ${ }^{1}$ J.M. Spikman²

${ }^{1}$ Martini Hospital, GRONINGEN, Nederland

${ }^{2}$ University Medical Center Groningen (UMCG), GRONINGEN, Nederland

Objectives: Virtual Reality as non-pharmacological pain relief during Wound Dressing Changes (WDCs) is promising, but needs to be fine-tuned to achieve a better effect. We studied the effect of VR across four WDCs on pain and need for pain medication. Thereby, the subjective feeling of being immersed in VR was evaluated. Furthermore, we investigated predictors of pain during the first WDC.

Methods: In the period October 2016 to December 2018, in- and outpatients of eight years and over, without need for intensive care and without physical limitations to use VR were randomly assigned to the VR or Control group. Both groups underwent four consecutive WDCs as usual and used pharmacological pain relief when needed. The VR-group also used VR during WDCs. VR-equipment was carefully adapted to burn centre requirements. Intention-to-treat analysis was performed.

Results: In total 39 patients were included of whom 22 were allocated to VR-group. The course of pain and use of pharmacological pain relief across four WDCs was not significantly different in both groups $(\mathrm{F}(3 ; 111)=1.68, \mathrm{p}=0.175$ and $\mathrm{F}(2.4 ; 84.9)=0.59, \mathrm{p}=0.582$ respectively). Mean pain score during first 
WDC was 1.2 points lower in VR-group and clinically significant ( $\geq 0.9$ points). Despite of satisfaction with the use of VR $(m=7.2)$, VR-group rated their feeling of being immersed as moderate $(m=6.0)$ and suggested VR-improvements such as offering more interactive VR-content. Immersion was not correlated with pain $(\mathrm{p}=0.637 ; \mathrm{p}=0.112 ; \mathrm{p}=0.933 ; \mathrm{p}=0.738)$. Pain during first WDC could not be predicted by VR, TBSA burned or State Anxiety $(\mathrm{F}(3 ; 35)=0.73$, $\mathrm{p}=0.539)$.

Conclusion: The absence of statistically significant VR-effect was presumably related to moderate subjective feelings of being immersed in virtual environment. In fine-tuning VR as pain relief, immersion should be maximised, for example by offering a wide range of VR-games.

O22.04 Prevalence and predictors of fatigue following burns: a prospective study

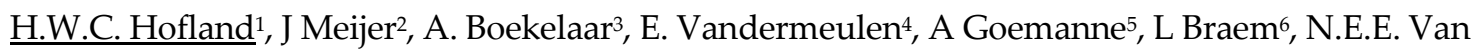
Loey $^{7}$

${ }^{1}$ Maasstadziekenhuis / ADBC, ROTTERDAM, Netherlands

${ }^{2}$ Martini ziekenhuis, GRONINGEN, Netherlands

${ }^{3}$ Rode Kruis Ziekenhuis, BEVERWIJK, Netherlands

${ }^{4}$ Els.vandermeulen@mi.be, BRUSSEL, Belgium

${ }^{5} \mathrm{UZ}$ Gent, GENT, Belgium

${ }^{6}$ ZNA STuivenberg, ANTWERPEN, Belgium

7ADBC, BEVERWIJK, Nederland

Objectives: Objectives: Studies that examined fatigue after burns are scarce and very few studies have investigated predictors of fatigue. Consequently, prevalence rates and predictors remain largely unclear. However, the small body of available literature indicates that between 25 and $50 \%$ of the patients reported fatigue 12 to 24 months post burn and anecdotic reports suggest that female gender and burn severity are associated with higher fatigue levels. The aim of this study was to prospectively document levels of fatigue between hospitalisation and 18 months later, and to examine early predictors of long-term fatigue.

Methods: Methods: A multicentre prospective cohort study including 248 adult patients at T1, mean age of 44 years old (SD=43), mean length of stay of 22 days ( $\mathrm{SD}=16)$, mean TBSA burned $10 \%$ $(\mathrm{SD}=11)$, completed the Multidimensional Fatigue Inventory (MFI) at 5 time points: during hospitalisation, 3, 6, 12 and 18 months post burn. Early predictors such as burn severity, perceived life threat, inhalation injury, age and gender, were assessed using linear regression analyses.

Results: Results: During hospitalisation, $46 \%$ reported moderate to severe levels of fatigue. Twelve and 18 months later, $24 \%$ and $22 \%$ of the patients respectively reported moderate to severe levels of fatigue. Early predictors of long-term fatigue were female gender, burn severity and the feeling one's life was in danger during the burn event. Age and inhalation injury were not statistically significant.

Conclusion: Conclusion: This study confirms that a considerable number of patients with burns experienced moderate to severe fatigue but, on average, levels of fatigue declined during the first year. Female gender and both physical and psychological factors increase the risk to develop long-term fatigue. The results of this study emphasise that fatigue should be a topic of interest during follow-up visits, and that that interventions should be considered in the group with stable high fatigue scores.

O22.05 The impact of the LDI on the anxiety levels in patients with burns

\section{G. Hartlief, A.S. Niemeijer, K.F. Lamberts, M.K. Nieuwenhuis}

Martini Hospital Groningen, GRONINGEN, Netherlands

Objectives: This study examines the effect of the Laser Doppler imaging-technique on patients' anxiety levels. The LDI-technique is non-invasive and supports staff in burn centres to determine whether a patient is in need of surgery. Its validity, reliability and cost effectiveness have been proven. A drawback of many techniques is the anxiety it evokes in patients. Uncertainty and/or anxiety are associated with physiological stress responses. Such responses are linked to poor coping with healthrelated issues, a decrease of quality of life, poor recovery and poor wound healing. . $^{1,2}$ We hypothesized that an inconclusive finding would result in elevated anxiety levels compared to those who received a conclusive decision. 
Methods: For this pre-test post-test study, patients were eligible if they had burns of indeterminate depth and were admitted to the burn centre or treated as outpatient. Two groups of patients were compared: the group that received an early decision about the best treatment $(n=45$; in need of surgery $n=17$ ) versus the group that had to wait for a decision $(n=14)$. Before and after the LDI was made and discussed with the patient, the patients scored their anxiety on a $100 \mathrm{~mm}$ visual analogue scale (VASA). The threshold for a clinical level of anxiety was $50 \mathrm{~mm} .{ }^{3}$

Results: In both groups, before the LDI image was made the median VAS scores were above 5 . After the LDI was made and discussed with patients, the medians were significantly lower: below 3 $(p=.001)$. No significant differences between both groups were observed $(p>.05)$.

Conclusion: In contrast to other studies, anxiety was significantly reduced in all our study groups after sharing the information. Communication of knowledge by health care professionals is important regardless whether the outcome is conclusive or not.

\section{References}

1)Mishel, 1984 pmid: 656794

2)Lang, Berbaum \& Lutgendorf, 2009 pmid: 19244038

3)Facco et al., 2013 pmid: 23860442

O22.06 Impulsiveness and burn patients

$\underline{\text { RJ Palmu }}^{1}$, T Partonen ${ }^{2}$, K Suominen $^{3}$, J Vuola $^{1}$

${ }^{1}$ Helsinki University Hospital, VANTAA, Finland

${ }^{2}$ National Institute for Health and Welfare, HELSINKI, Finland

${ }^{3}$ Department of Mental Health and Substance Abuse, HELSINKI, Finland

Objectives: Impulsiveness is a tendency to act quickly based on a whim without reflection or consideration of consequences. We studied its correlations with burn variables and mental disorders among burn patients.

Methods: Consecutive acute burn patients (N=107) admitted to the Helsinki Burn Center were assessed with the Structured Clinical Interview for DSM-IV mental disorders (SCID) at baseline and at 6 months. All patients filled out the 30-item Barratt Impulsiveness Scale (BIS-11), the most commonly administered self-report measure and a standard point of reference in research on impulsiveness.

Results: The mean total score of BIS-11 was 64.5 (range 41.0-87.8, SD \pm 6.9 ). There was not a significant correlation between impulsiveness and a range of characteristics of burn injury (all p-values $>0.05$ ). We found a significant correlation between some pre-burn mental disorders and impulsiveness, alcohol dependence and attentional impulsiveness $(\mathrm{OR}=1.22, \mathrm{p}=0.022)$, any personality disorder and non-planning impulsiveness $(\mathrm{OR}=1.21, \mathrm{p}=0.005)$, and antisocial personality disorder and motor impulsiveness $(\mathrm{OR}=1.35, \mathrm{p}=0.043)$. Patients with high impulsiveness (total score $>65)$ more often than those with low impulsiveness $(\leq 65)$, had pre-burn mental disorders such as major depressive disorder (22.6\%vs. 8.6\%, $\mathrm{p}=0.046)$, alcohol dependence ( $46.9 \%$ vs. $25.9 \%$, $\mathrm{p}=0.023)$, or other substance dependence (12.2\% vs. $1.7 \%, \mathrm{p}=0.028)$.

Conclusion: Impulsiveness had a significant correlation with mental disorders but not with burnrelated variables. Therefore the role of impulsiveness in burn injuries should not be investigated independently without first accounting for the role of mental disorders.

\section{O22.07 Self-Immolation in Tunisian Adolescents: A 16-Year Study}

M BEN SAAD, M SOMAI, K FELAH, L DEBBICHE, A AYARI, H FREDJ, O GASRI, I Jami, A MOKLINE, AA MESSADI

Burn Center of Ben Arous, BEN AROUS, Tunisia

Objectives: Following the revolution of 2011, Tunisia witnessed a wave of copycat suicides that reached epidemic proportions. The aim of this study is to highlight the peculiarities of self-immolation in tunisian adolescents over a period of 16 years (8 years before and 8 years after the revolution).

Methods: This is a descriptive retrospective study of tunisian adolescents aged 10-19, who selfimmolated then were admitted to tour intensive Burn care in Tunisia, from January 2003 to December 2018. Patients diagnosed with accidental burn or burns from undetermined origin were excluded from 
the study.

We examined epidemiological and clinical characteristics of the patients.

Clinical data were collected from burn patients' medical records.

Study variables included: gender, age, place of residence, year of admission, motive, TBSA, burned parts of the body, the depth of burns, and mortality.

Results: During the study period, 5027 patients were admitted to our department. 516 were aged 10-19. Of those, 73 (14\%) were self-burned.

Gender ratio was 3,56.

Fourty two percent of the patients were out of school. Fifty six percent came from poor families, $41 \%$ from modest families, and $3 \%$ from rich families. The average number of brothers and sisters was $2,5$ (1 to 7$)$.

Self-immolation was more frequent in adolescents residing in urban places $89 \%$.

A peak of incidence was seen in 2011. In that year alone, we had more cases than in the eight previous years combined.

Ten patients (13,5\%) self-immolated before January 2011 and 63 (86,5\%) 2011.

The annual rate of self-immolations was 1,25 patients per year before 2011 and 7,87 patients per year after 2011.

The prevailing motive of self-immolation was family conflict $(n=25)$, followed by school problems $(\mathrm{n}=8)$.

Conclusion: Adolescents who self-immolated were mostly males from economically disadvantaged families and living in urban places. The main reasons for self-immolation were family conflicts and school problems.

\section{O22.08 Social work in Helsinki Burn Centre}

K.A. Kokkola, K.M. Lumilampi

Helsinki University Hospital, HUS, Finland

Objectives: In January 2016 the new national Helsinki Burn Centre opened at the Jorvi hospital. Since the beginning, social work has played a major role in the rehabilitation process of burn patients. Two social workers in the burn centre offer social work services for both patients and their family members.

Social workers are part of the multi-professional team, which, in addition to providing acute health care, plans and organizes the rehabilitation and discharging of the patient. The work includes psychosocial support and crisis intervention, coordination of social services and providing information and help with social benefits, insurances and legal issues.

The aim of this presentation is to give an overview of social work at different levels: the Finnish society and social security system, health care, burn centre and individual level of patients.

Methods: The presentation focuses on demonstrating the complex and demanding work of social workers in specialized health care and especially in the national burn centre.

Results: Several social work themes are essential in the burn unit: Finnish social security system, working with patients in different age groups, different social problems (i.a. child welfare questions, substance abuse problems, and domestic violence), mental health issues, rehabilitation, legal issues and post-discharge services.

Conclusion: Social work in the national burn centre is an essential part of the rehabilitation process because severe burn injuries often have long-term psychological and social effects on patients' lives. Burn patients also often have had mental health and social problems already before the burn injury. Widespread knowledge of social services and social benefits and psychological capacity to work with demanding group of patients are required from burn centres social workers.

O23.01 Burns of the feet in children.

BABUR Shakirov ${ }^{1}$, K Karabaev $^{2}$, R Tagaev², A Hakimov' ${ }^{1}$, S Mavlyanova ${ }^{2}$

${ }^{1}$ Republican Scientific Centre of Urgent Medical Aid (RSCUMA), SAMARKAND, Uzbekistan

2Samarkand state Mesical Institute, SAMARKAND, Uzbekistan 
Objectives: Foot burns in a pediatric patient require special consideration. Although the feet involve a small percentage of the total body surface area, they can have major effects in daily life, caused by prolonged bed rest, hospitalization and high risk of both early and late complications. In Central Asia foot burns are widespread, because many people, especially children, walk barefoot in summer, and because the heated sandal is still used for keeping warm in winter.

Methods: This is a retrospective study of 132 patients who were admitted at the Burns department of Republican Scientific Centre of Urgent Medical Aid (RSCUMA). Most of them were children under 3 years of age who fell into sandal heaters. The following features characterized the pathogenesis of foot burns. First, sandal and hot ash burns were caused by direct contact between skin and thermal agent. Second, in all cases, edema of the soft tissues of the feet developed during the first 24-48 $\mathrm{h}$ after thermal burns. To prevent this, the patients with recent burns were treated with necrotomy. Chemotherapeutic materials were placed on the wound surface to accelerate rejection of the necrotic tissues. Necrectomy was performed as early as 7 to 9 days after the burn incident if the burned surface area, did not exceed $5 \%$ to $7 \%$ of the patient's total body surface area. We normally placed skin grafts on granulation tissue, when a wound was completely ready for auto graft closure.

Results: Of all patients, 76, 4\% were healed after the first autodermoplastic surgery. The second autodermoplastic surgery was performed $23,6 \%$ of the patients because the transplanted skin dissolved in some places.

Conclusion: This effective treatment method contributed to the restoration of foot function in patients, lessened their deformities and post-burn contractures $\backslash$ deformities.

O23.02 Pediatric Burns: A descriptive study of Specialized Burns Unit in Catalonia (2010-2018

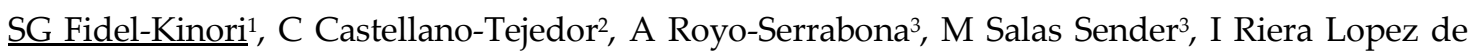
Aguileta ${ }^{3}$, MS Cepeda-Diez ${ }^{1}$, JP Barret-Nerin ${ }^{1}$

${ }^{1}$ Hospital Universitario Vall d'Hebron, BARCELONA, Spain

${ }_{2}^{2}$ RE.FIT Research group Parc Sanitari Pere Virgili, BARCELONA, Spain

${ }^{3}$ Parc Sanitari Sant Joan de Dèu, SANT BOI DE LLOBREGAT, Spain

Objectives: (1) to describe pediatric burns admitted to a reference specialized burn unit (SBU) in Catalonia (Spain), between 2010-2018 and (2) to provide a preliminary descriptive profile of this sample population to be compared to other SBUs from Europe

Methods: Between 2010-2018, socio-demographics (age, gender, nationality) and data related to the injury (aetiology, setting, total body surface area (TBSA), evolution) and clinical aspects of the patient (psychopathological diagnosis and background), were collected from a sample of 496 pediatric patients ( $<=16$ years old) in a cross-sectional study

Results: Mean age of the whole sample was 3,9+-4,11 years and most of them were males $(n=298$, $60,1 \%$ ) from Sapin ( $n=435,87,7 \%$ ). Psychopathological background was rarely found, both in family and the patient $(0,8 \%$ and $1,9 \%$, respectively). The majority of patients $(91,9 \%)$ were at home with their parents when the incident occurred. Mode of injury was by mean of hot liquids (scalds burns) in $84,3 \%$ cases and average of TBSA was around $8,24+-8,33 \%$ with $45,8 \%$ patients suffering from chirurgical wounds. Mean stage at the SBU was 14,60+-20,04 days and destination at discharge was mainly home $(94,2 \%)$ with less than 1 readmission on average for subsequent complications $(0,20+-0,75)$. No differences between genders were observed for any of these features.

Conclusion: The aim of this study was to provide recent and novel epidemiological data on pediatric burns from a reference hospital in Catalonia (Spain) with the final aim of suggesting future preventive strategies. Profile regarding socio-demographics and etiological data in this sample are comparable with the characteristics of most European countries. According to EuroSafe report, prevalence of pediatric burns has increased in Spain despite being in the lower third, together with Finland, Austria, Sweden and Greece. This population requires specific rehabilitation resources and accurate monitoring of the evolution of physical and psychosocial sequelae, because they differ from adult burns.

O23.03 Error analysis of the first aid in children with burn on prehospital stage 
D Oborkina, L Budkevich, D Dolotova, T Zagriazkina

Pirogov Russian National Research Medical University, MOSCOW, Russia

Objectives: The purpose of this study was the need to identify the most significant moments in the provision of first aid to children with a burn injury at the stage of evacuation to a specialized hospital.

Methods: A retrospective study of 328 patients aged from 0 to 17 years and 11 months who were admitted to a burn center for a three-year period from 2016 to 2018. The study included children with a burn injury delivered to a specialized hospital by the emergency medical team with signs of shock and requiring hospitalization in the intensive care unit.

Results: The average age of the patients was 16 months, the evacuation time was 67 minutes, the duration of hospitalization was 12 days. Further surgical treatment required $44.8 \%$ of patients. Of the 328 people, only 240 (73\%) were identified burn area. Cases of hyper and hypodiagnosis were $27 \%$ and $41 \%$, respectively, in cases of hyperdiagnostic the area of established before hospitalization was significantly higher. Inadequate anesthetic therapy was observed in $61.9 \%$ of cases, of which $4.3 \%$ overdose, a shortage of $56.7 \%$, situations in which parents refused to undergo anesthesia $(2.1 \%)$ were taken into account. In the group of children for whom the area was not determined, errors with anesthesia were observed significantly more often.

Conclusion: The area of burn wounds is a fundamental criterion in assessing the severity of the condition and the choice of further patient management tactics. An error in the calculation of this indicator at the prehospital stage leads to an incorrect calculation of the amount of necessary analgesic therapy, an incorrect assessment of the severity of the condition and ultimately non-recognition of shock, which may adversely affect the course of the wound process and worsen the outcome of the disease.

\section{O23.04 Treatment of Deep Burns in Children by Utilizing Microsurgical Flaps}

\section{$\underline{\text { P. Gnipov }}{ }^{1}$, A. Baindurashvili2', S. Golyana' ${ }^{2}$, M. Brasol', E. Mitrofanova ${ }^{1}$, N. Zaytseva ${ }^{2}$}

${ }^{1}$ St-Petersburg Children State Hospital No. 1, SAINT-PETERSBURG, Russia

${ }^{2}$ The Turner Scientific Research Institute For Children's Orthopedics, SAINT-PETERSBURG, Russia

Objectives: To demonstrate results of treatment deep pediatric burns, by utilizing microsurgical flaps.

Methods: Pediatric burn department of Children State Hospital No. 1 of St-Petersburg, is the only one specialized unit for children with thermal trauma in North-West region of Russia. It works since 1971 and was established by specialists from The Turner Scientific Research Institute For Children's Orthopedics led by Kazantseva N. Since establishment, specialists of the pediatric burn unit help 900 children a year who suffered from burns. In last few years deep electric burns became problem for our region: children climb on the top of high-voltage towers and riding roofs of trains for photos on smartphones and sometimes these actions lead to dramatic results.

Each year our department cures 3-5 children with deep burns from high voltage electricity. And without microsurgery its almost impossible to restore damaged tissue. We want to demonstrate you few cases of pediatric deep burns, treated by utilizing different microsurgical flaps. All kids that transferred to our department were with electric burns.

Results: The results of treatment we obtained, confirms the prospects for further development and the indispensability of microsurgical flaps to cover defects caused by deep burns in children.

Conclusion: Microsurgical flaps can help to cover defects, where bones, joints, ligaments or nerves are visible in wound bed. In some cases even microsurgery couldn't help, and electricity damage leads to amputation of limbs. If there is a way to solve surgical problem, by using less risk surgical approaches- use them.

O23.05 Experiences in using a Biosynthetic membrane and Laser Doppler in children with scalds.

T K Randawa, J Stallard, PM Muthayya, A Phipps, U Anwar, C Swales

Pinderfields Regional Burns centre, WAKEFIELD, United Kingdom

Objectives: EpiProtect is a biosynthetic membrane which is free from human or animal product. 
We have been using it in children presenting to our centre with scald injuries. We aim to present our experiences in our first fifteen cases and the benefits in conjunction with the Laser Doppler.

Methods: Children presenting to the burn centre with significant scald injury. EpiProtet applied instead of other skin substitutes. Laser Doppler performed through the biosynthetic membrane 48 hours post injury at their initial dressing check.

Results: First fifteen patients presented. Majority of the patients had the dressing applied in the outpatient clinic, one child required a general anaesthetic due to pain and size of the burn. Laser Doppler preformed through the dressing 48 hours post injury, majority predicted healing in less than fourteen days. Laser Doppler predication accurate. No patients had a wound infection.

Conclusion: EpiProtect is a relatively new epithelium substitute to the market. There are multiple skin substitutes on the market. We regularly use skin substitutes in our paediatric scalds for the analgesic benefits.

We chose to use EpiProtect as we were able to apply it in our outpatient setting with ease.

Furthermore, EpiProtect to our knowledge is the only skin substitute on the market that allows laser penetration. This enabled us to use the Laser Doppler through the dressing. We have found the Laser Doppler to be an effective tool when counselling patients and their parents post burn injury. This dressing enables the Laser Doppler to be performed with minimal distress or pain to the child given the dressing remains intact.

To date our experiences with EpiProtect have been positive and we have found the ability to use the Laser Doppler through the dressing extremely useful. We hope to continue to use this dressing in our paediatric burns.

O24.01 Donor site dressings: how much do they affect pain?

\section{Grigg, J Brown, C Town}

Royal Brisbane and Women's Hospital, HERSTON, Australia

Objectives: Pain causes significant distress in burn patients and is both neuropathic and nociceptive in character. Background pain is usually managed with regular, long-acting medications whilst dressing changes require breakthrough analgesia. Dressing changes remain a substantial source of acute pain but are a necessary evil. This audit focuses on Suprathel ${ }^{\circledR}$ used specifically in donor sites and the effect it has on both analgesia and opiate requirements.

Methods: Data was collected retrospectively over a three-month period at the Royal Brisbane and Women's Hospital Professor Stuart Pegg Adult Burns Centre. All patients who received Suprathel ${ }^{\circledR}$ (under the discretion of the consultant surgeon) and had small burns $<5 \%$ TBSA were included in the audit. This data was then compared to previously collected data involving patients who had standard dressing care - Algisite. Pain scores and opiate requirements were then compared between the two.

Results: Patients length of stays was reduced by $50 \%$ in the Suprathel ${ }^{\circledR}$ group ( 4.05 days vs 8.78 days). Opiate requirements day 1 post-operatively were three times in the standard dressings group compared to Suprathel ${ }^{\circledR}(58 \mathrm{mg}$ vs $21 \mathrm{mg}$ of oral morphine) and remained greater through to Day 7 postoperatively.

Conclusion: This audit demonstrates the importance of choosing appropriate dressings and the benefits of pain reducing dressings. Suprathel ${ }^{\circledR}$ is emerging as an alternative to standard dressings as both a way of reducing dressing changes and improving analgesia.

O24.02 A study on changes of pancreas structure and function after severe burns and its mechanism

\section{CA Shen}

The Fourth Medical Center of Chinese People's Liberation Army General Hospital, BEIJING, China

Objectives: This study aimed to explore the changes of pancreas structure and function after severe burns and its mechanism.

Methods: A scald model of rats with a $50 \%$ total body surface area was established. Rats were injected with exenatide (Exendin- $4,4 \mu \mathrm{g} / \mathrm{kg}$ ) subcutaneously twice daily post scald creation. Peripheral blood glucose, glucagon-like peptide 1 (GLP-1) and insulin concentration were measured to calculate 
the insulin resistance index at $72 \mathrm{~h}$ post injury. Magnetic resonance imaging (MRI) was used to observe the morphology and structure of the pancreas. Insulin immunofluorescence staining was employed to examine the functional and structural changes of pancreatic tissue under light and electron microscopy. Islet cells were isolated from the pancreas and incubated in vitro with low-concentration $(2.8 \mathrm{mmol} / \mathrm{L})$ glucose and high-concentration ( $16.7 \mathrm{mmol} / \mathrm{L}$ ) glucose RPMI 1640 medium for 1 or 24 hours. The insulin secretion index (SI) and the number of insulin particles and insulin vacuoles attached to the cell membrane were calculated. The content of insulin and proinsulin in the islet cells were detected by immunoblotting, and the expression of insulin mRNA by RT-PCR.

Results: Scalds increased fasting blood glucose (FBG), lowered serum GLP-1 levels and impaired glucose tolerance significantly in the rats. Exendin- 4 could reduce blood glucose, increase GLP- 1 and improve glucose tolerance remarkably. MRI showed the rats' pancreatic tissue was edematous obviously after scalds. Due to the stimulation of high-concentration glucose, the insulin secretion was reduced significantly, so was the content of insulin, proinsulin and insulin mRNA. Extendin- 4 could increase the secretion of insulin and improve insulin mRNA levels.

Conclusion: Exendin- 4 can significantly improve the structure and function of $\beta$ cells in the islets of scalded rats and lower their blood glucose levels.

O24.03 The Use of ImageJ to Calculate Total Body Surface Area in Burn Patients: A Case Series

R.A. Widyana, R. A. Widyana, G. A. Winarno, S. F. Tanjunga, R. A. Adiwiria

Cipto Mangunkusumo Hospital, JAKARTA, Indonesia

Objectives: Assessing the total body surface area (TBSA) of burn has been a matter of clinical judgment even though some modalities or applications may serve as adjunct tools to increase the accuracy in determining TBSA. We tried to compare the result between clinical judgment from an experienced burn surgeon ( $>10$ years in the field) and manual calculations using ImageJ.

Methods: We conducted the study in the burn unit of Cipto Mangunkusomo Hospital, Jakarta. Using transparent dressing, the border of each burn wound is traced and the area is measured using "ROI Manager" mode in ImageJ. The overall BSA of each patient was calculated using the Dubois Formula as the denominator to determine the percentage of burn area. The percentage from ImageJ calculation is then compared to the Rule of Nine.

Results: Data was collected in the first day post injury of 3 patients, all with partial to full-thickness burn. Patient A was a normoweight female with 36\% burn clinically and 34.7\% from ImageJ. Patient B was an obese female with $25 \%$ burn clinically and $32 \%$ from Imagej. Patient $C$ was an overweight female with $10 \%$ burn and $8.5 \%$ from ImageJ. In 2 patients, measurements are identical with $<2 \%$ difference. The exception in 1 patient with $7 \%$ difference may be caused by obese proportion.

ImageJ provide detailed decimal number but with a setback in circular body parts such as arms or legs. Dressing could not attach precisely to the wound for accurate tracing. ImageJ is useful in a noncircumferential wound or when a clinician needs an exact wound area for research or trials.

Conclusion: ImageJ serves as an aid to calculate wound area but cases are different in burns with unpredictable and circumferential wound. An attempt to measure BSA using ImageJ is not futile but not more beneficial than using the common rule of nine.

O24.04 Reviving the Burn Scoring System: An Aid for Appropriate Patient's Care in Cipto Mangunkusumo General Hospital, Jakarta, Indonesia

A. Wardhana, S. Tanjunga, G. Winarno, R. Widyana

Cipto Mangunkusumo General Hospital, DKI JAKARTA, Indonesia

Objectives: This study aims to predict the outcome in burn patients in Cipto Mangunkusumo General Hospital using burn scoring system

Methods: In this retrospective cross-sectional study, we collected data from health record, a total of 513 patients between 2013-2017 and ran them into four scoring systems: Modified Baux, ABSI, BOBI, and Ryan Score. The final results are probability of mortality or survival of each patients and we compare them into the real results during inpatient treatment. Patients are classified as healed according to the discharge criteria in our hospital before being moved to outpatient treatment. The 
exclusion criteria for this study are children ( $<18$ years old), patients admitted $>7$ days post injury, chronic burns, electrical and chemical burns.

Results: The cut-off value for ABSI is 8 , Ryan is 1 , BOBI is 4, and Modified Baux is 93. For the ABSI score, the AUC is 0.772 (CI 95\%, 0.725-0.819) with p-value of 0.000. For the Ryan Score, the AUC is 0.593 (CI 95\%, 0.537-0.649) with p-value of 0.001. For the BOBI score, the AUC is 0.582 (CI 95\%, 0.527-0.638) with p-value of 0.003. For Modified Baux Score, the AUC is 0.755(CI 95\%, 0.705-0.805) with p-value of 0.000 .

Conclusion: All the scoring systems can be used in our unit based on the p-value but the best one is the ABSI score. Knowing this may be helpful to determine the standard of care for each patient to maximize the available resource.

O24.05 FLIR One as an Adjunct Tool for Burn Wound Depth Assessment

A Wardhana, R. A. Adiwiria, R Widyana, S Tanjunga, G Winarno

Cipto Mangunkusumo Hospital, JAKARTA, Indonesia

Objectives: Assessing burn wound depth has been a matter of clinical judgment for years until adjunct tools started to appear to increase the accuracy of a physician's judgment. FLIR One ${ }^{\circledR}$ serves as an easier tool to use due to its size and portability. We tried to measure burn wound depth using FLIR One ${ }^{\circledR}$ by making the association to wound temperature on day 1 and 3 when the burning process is still happening.

Methods: We collected data from 4 patients in Cipto Mangunkusumo General Hospital Burn Unit admitted in February 2019. Thermal imaging data on the $1^{\text {st }}$ and $3^{\text {rd }}$ day of burn injury was obtained then compared to each patient's examinations. White wound means deeper burn and is equal to lower temperature while reddish wound is more superficial and equal to higher temperature.

Results: The observation on 4 patients shows that thermal images from all patients matched the wound appearances and the relation is as expected. Low temperature leads to deeper burn and surgery. Patient $A$ and $B$ with higher thermal image on the $1^{\text {st }}$ and $3^{\text {rd }}$ day of burn injury did not undergo a burn wound excision. Patient $C$ and D with lower temperature on the $1^{\text {st }}$ and $3^{\text {rd }}$ day was operated on the $5^{\text {th }}$ day.

Conclusion: It shows that lower temperature on the $1^{\text {st }}$ day of injury indicate a deeper wound and bigger probability of surgery. The $1^{\text {st }}$ day results have higher accuracy compared to the $3^{\text {rd }}$ day results due to minimal physiologic change. Further study of FLIR ONE assessment accuracy on burn wound patient would be needed.

O24.06 Unravelling the mystery of Lichtenberg Figures

A Lindford ${ }^{1}$, S Juteau ${ }^{1}$, V Jaks'2, M Klaas ${ }^{2}$, H Lagus ${ }^{1}$, J Vuola ${ }^{1}$, E Kankuri ${ }^{3}$

${ }^{1}$ Helsinki University Hospital, HELSINKI, Finland

${ }^{2}$ Unit of Cell Biology, University of Tartu, Estonia, TARTU, Estonia

${ }^{3}$ Department of Pharmacology, Faculty of Medicine, University of Helsinki, Finland, HELSINKI, Finland

Objectives: Lichtenberg figures (LFs) are a pathognomonic skin sign for a lightning strike injury. The true pathophysiology of LFs remains unknown and numerous theories have previously been proposed. We describe two cases of LFs; one following a lightning strike and the other following a high voltage electrical injury. A skin biopsy taken directly from the LF-affected site in the latter patient was then used to perform proteomics and immunohistochemistry in an attempt to further unravel the molecular and histological aspects contributing to the pathobiology of the tissue response.

Methods: A 4mm punch biopsy was taken from the erythematous area of LFs. A similar biopsy was takenat 3 months from the same fully healed location. Both tissue samples were analysed histopathologically and underwent both proteomic testing and immunohistochemical staining. Protein expression was ultimately compared between the site of injury containing the LFs and 3-month control samples.

Results: Histopathological analysis revealed prominent lymphocytic infiltrates around the small blood vessels in the upper dermis of the injury sample. Red blood cell extravasation was also seen. 
Periodic acid-Schiff (PAS) staining showed intact vessel walls and absent vasculitis, but lymphocytic infiltration was still seen. Proteomic analysis identified certain proteins such as lactoferrin and smooth muscle proteins at a higher abundance in the injured tissue in contrast to the control tissue. Immunohistochemical staining patterns for anti-lactoferrin revealed epithelial cells and endothelial cells staining positive for lactoferrin.

Conclusion: We have revealed certain upregulated proteins that can provide a feasible explanation for this mysterious transient phenomenon. Our findings suggest that LFs are indeed a pathological as well as physical phenomenon, resulting from injury to the skin from high voltage electricity or a lightning strike. Of particular interest is the expression of lactoferrin and its likely role as an Alarmin in the inflammatory response.

O24.07 Development of an ex-vivo burn wound model using full thickness skin organ culture

$\underline{\text { M Shah }}^{1}$, J Oldbury ${ }^{1}$, A Shahid ${ }^{2}$, A Thomlinson ${ }^{2}$

${ }^{1} \mathrm{RMCH}, \mathrm{MANCHESTER}$, United Kingdom

${ }^{2}$ University of Manchester, MANCHESTER, United Kingdom

Objectives: (1) To optimise ex vivo full thickness porcine and human skin organ culture of varying diameters and (2) To develop standardised ex-vivo "burn wound healing" model of varying depths to study re-epithelialisation

Methods: Porcine skin obtained from abbatoir and human skin from consented patients undergoing breast reconstruction (with approrpiate ethical approval) was transported in Dulbeccos modified eagles medium (DMEM) on ice. After washing with alcoholic chlorhexidine and phosphate bufferd saline, discs of $10 \mathrm{~mm}, 15 \mathrm{~mm}$ and $20 \mathrm{~mm}$ diameter were cut from samples and organ cultures set in 6 well plates by placing the skin on sterile gauze submerged in supplemented DMEM with the epidermis at air-liquid interphase. Culture medium was chaged every other day.

Burn wounds were created by aluminium rods heated to $70 / 80^{\circ} \mathrm{C}$ for $30 / 20 \mathrm{sec}$ to form superficial partial thickness or mid-dermal burn wounds respectively.

Samples were harvested at 7, 11, 14 or 21 days post-cutlure. Samples were processed for cryosectioning/paraffin embedding and subjected to routine histology or immunostaining and wholemounts to study proliferation (PCNA), apoptosis (Tunel), keratin expression (K14) and laminin for basement membrane. Samples were photographed and subjected to image analyses.

Results: $1.5 \mathrm{~cm}$ diameter full thickness porcine and human skin could be sustained as organ cultures with and without a burn wound upto day 14 with minimally supplemented DMEM (no growth factors). Re-epithelialisation and basement membrane formation was also seen in ex-vivo burn wound model. Wholemounts immunostained with K14 demonstrated the advancing re-epithelialisation.

Conclusion: We were able to maintain full thickness porcine and human skin organ cultures with or without burn wounds viable on minimally supplemented culture media for atleast 14 days. This burn wound model can be used to study re-epithelialisation ex-vivo and in the future can be used to test treatments targetted at acceleration of re-epithelialisation in burn wounds.

O26.01 Results of the DETECT enzymatic debridement multicenter randomized controlled trial

Y Shoham ${ }^{1}$, E Pagnozzi², I Mataro², A Stancioiu³, B Lipovy ${ }^{4}$, G Kashibadze ${ }^{5}$, S Monstrey ${ }^{6}$, S Marinescu ${ }^{7}$, A Boiangiu 7 , B Hartmann ${ }^{8}$, F Sander 9 , L Rosenberg ${ }^{10}$

${ }^{1}$ Soroka University Medical Center, BEER SHEBA, Israel

${ }^{2}$ Cardarelli Hospital, NAPLES, Italy

${ }^{3}$ Spitalul Clinic de Urgenta de Chirurgie Plastica, BUCHAREST, Romania

${ }^{4}$ Klinika popálenin a rekonstrukcní chirurgie, BRNO, Czech Republic

${ }^{5}$ Khechinashvili University Hospital, TBILISI, Georgia

6University Hospital, GENT, Belgium

${ }^{7}$ Spitalul Clinic de Urgenta „Bagdasar-Arseni,,, BUCHAREST, Romania

${ }^{8}$ Unfallkrankenhaus, BERLIN, Germany

'Unfallkrankenhaus Berlin, BERLIN, Germany

${ }^{10}$ Meir Medical Center, KFAR SABA, Israel 
Objectives: To evaluate the efficacy and safety of NexoBrid.

Methods: 175 patients suffering from deep burns were included in a multicenter, multinational, randomized, controlled, assessor blinded trial. Patients were randomized to 3 arms - NexoBrid, Standard of Care (SOC), or Gel vehicle in a 3:3:1 ratio. The primary endpoint was incidence of complete debridement (NexoBrid vs Gel). Additional acute stage endpoints included the need for surgical debridement, time to complete debridement, blood loss and time to complete wound closure (NexoBrid vs SOC).

Results: Patient demographics and wound baseline characteristics were comparable across study arms. The incidence of complete debridement was significantly higher for NexoBrid vs Gel patients (93.3\% vs $4 \%, \mathrm{p}<0.0001)$. The need for surgical debridement was significantly lower for NexoBrid vs SOC patients ( $4 \%$ vs $72 \%, \mathrm{p}<0.0001$ ). The median time to complete debridement was significantly shorter for NexoBrid vs SOC patients ( 1 day vs 3.8 days, $\mathrm{p}<0.0001)$. Calculated blood loss was significantly lower for NexoBrid vs SOC patients $(14 \mathrm{ml}$ vs $815 \mathrm{ml}, \mathrm{p}<0.0001)$. The median time to complete wound closure was similar for NexoBrid vs SOC patients ( 27 vs 28 days). The overall safety profile of NexoBrid treated patients was good, and consistent with the safety data known from previous studies. Long term follow up results are not yet available.

Conclusion: The results of this robust phase 3 trial demonstrate the safety and efficacy of NexoBrid, and are in line with former trials results.

O26.02 Enzymatic debridement for burn injury of the hand - a single center experience

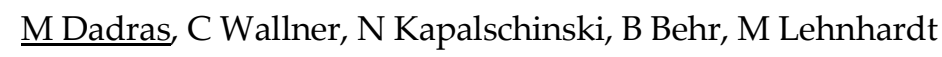

Berufsgenossenschaftliches Universitätsklinikum Bergmannsheil Bochum, BOCHUM, Germany

Objectives: Burn injury to the hand poses a challenge due to anatomic complexity and functional relevance. Enzymatic debridement with Nexobrid ${ }^{\circledR}$ has been introduced as a therapeutic option with the aim of preserving viable tissue and improving healing.

Methods: A retrospective analysis of patients with burn injury of the hand treated with Nexobrid ${ }^{\circledR}$ between January 2014 and January 2019 at our institution was performed. Demographic, clinical and outcome data were collected and analyzed.

Results: A total of 44 patients could be identified. 35 patients were male and 9 female. Age ranged from 20 to 90 with a median of 47 . TBSA was $15,6 \pm 18 \%$. Death occurred in one patient due to sepsis with multi organ failure. 17 of the patients $(38 \%)$ needed operative debridement and split thickness skin grafting of the hand due to partial full thicknes burn or delayed healing. This included 9 of 14 patients (64\%) treated from 2014 to 2016 and 8 of 30 patients (27\%) treated from 2017 to January 2019.

Conclusion: Enzymatic debridement represents a valuable treatment option for deep dermal burn injury of the hand. A learning curve in the selection of patients and timing of needed operative intervention could be observed in our department leading to good results and potentially reduction of need for skin transplantation.

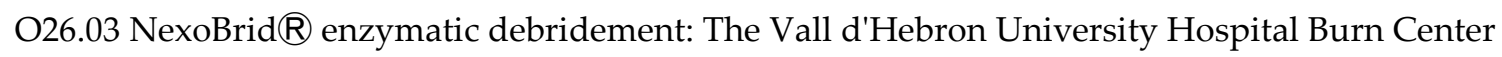
experience

E. Verdaguer Olivella, A Monte Soldado, J Serracanta Domènech, J Aguilera Sáez, J. M. Collado Delfa, J.P. Barret Nerín

Vall d'Hebron University Hospital, BARCELONA, Spain

Objectives: To review the evolution of NexoBrid ${ }^{\circledR}$ usage over 4 years in a tertiary Burn Center, describing changes in indications, post-debridement dressings, and surgical management over debrided wounds.

Methods: Review of digitalized medical records and descriptive analysis from the year 2015 to 2019.

Results: Between 2015 and 2019, NexoBrid ${ }^{\circledR}$ was performed in 164 cases, of which 78\% were men and $22 \%$ were women, with an average age of 45 (in a range from 17 to 94 years old).

In regard to selection criteria, NexoBrid ${ }^{\circledR}$ has proved to be a painful treatment which requires adequate pain control. We currently exclude patients with a suspected low pain threshold due to 
substance abuse or previous comorbidities.

$98 \%$ of the treatments have been performed in burns placed in extremities, as pain management by means of regional anesthesia is easier in this location.

Once debridement is completed, the dressings used in our Unit might differ from the scientific consensus (Hirche et al., 2017): In cases that show an intermediate appearance, we would routinely use a synthetic skin substitute (Suprathel $\left.{ }^{\circledR}\right)$, avoiding the need for surgery and a shorter hospital stay. For deeper lesions, which are likely to require surgical treatment, we would avoid silver sulfadiazine as it impairs the assessment of the wound bed, and would use Nitrofurazone dressings instead. Recent evidence has shown that after enzymatic escharolysis with NexoBrid ${ }$, removal of necrotic tissue appears to be incomplete in the wound bed (Di Lonardo et al., 2018). For that reason, we now perform a superficial tangential surgical debridement before grafting.

Conclusion: We have adjusted the use of NexoBrid ${ }^{\circledR}$ according to our experience and the emergence of new scientific evidence, resulting in the enhanced overall management of deep and intermediate burns and improved care of our patients.

O26.04 Enzymatic debridement: a retrospective study to evaluate the accuracy of burn depth diagnosis before and after treatment?

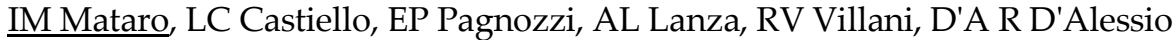

Hospital A. Cardarelli, NAPLES, Italy

Objectives: The most common technique to determine burn depth is clinical assessment by experienced burn surgeon, although this has been shown to be accurate in only $60-75 \%$ of the cases. Enzymatic debridement (ED) is considered one promising alternative of non-surgical option for eschar removal and results suggested that the use of this technique reduces need for surgery and autografting. We present a retrospective study to evaluate the accuracy of burn depth diagnosis before and after enzymatic debridement

Methods: Between January 2016 and June 2018, we collected the data regarding burn depth of patients with thermal burns treated with enzymatic debridement. For each patient a target burn was identified. Senior burn specialists were asked if the burns required graft or no before ED and after ED. Using the final treatment as a gold standard, the sensitivity, specificity, positive and negative predictive value of the clinical diagnosis (both pre- and post-treatment) were calculated

Results: The study sample consists of 69 burn patients and $31.9 \%$ of the sample ( $n=22 / 69$ ) underwent skin grafting; analysing K-agreement, a $40.6 \%$ discrepancy between the pre and posttreatment is observed (kappa $=0.3 ; \mathrm{p}=0.000$ ). The sensitivity of the clinical assessment pre- treatment is $86.4 \%$, the specificity $40.4 \%$, the positive predictive value $40.4 \%$ and the negative predictive value $86.4 \%$. The sensitivity of the clinical assessment post-treatment is $86.4 \%$, the specificity $100.0 \%$, the positive predictive value $100.0 \%$ and the negative predictive value $94.0 \%$.

Conclusion: An accurate diagnosis of burn depth is a vital goal in the management of burn patient: overestimation of burn depth can have heavy consequences since they lead to unnecessary surgery. The direct visualization of dermal vital tissue after enzymatic debridement allows a more accurate burn depth diagnosis reducing need for surgery and autografting with satisfactory long term results.

Picture 1: https://www.eventure-online.com/parthen-uploads/89/9EBA/add_1_539602_b95b781d6c39-4b77-8af1-4b495fae52ac.png

Picture 2: https://www.eventure-online.com/parthen-uploads/89/9EBA/add_3_539602_b95b781d6c39-4b77-8af1-4b495fae52ac.png

Picture 3: https://www.eventure-online.com/parthen-uploads/89/9EBA/add_539602_b95b781d6c39-4b77-8af1-4b495fae52ac.png

Picture 4: https://www.eventure-online.com/parthen-uploads/89/9EBA/add_2_539602_b95b781d6c39-4b77-8af1-4b495fae52ac.png

O26.05 Obtaining government refund for NexoBrid: results from the NEXOBRID Belgian Registry

E.Y. Claes

Ghent University Hospital, GENT, Belgium 
Objectives: Belgium is the first country where burn centers succeeded in obtaining government refund for the use of the debriding enzyme NexoBrid (NXB). This paper illustrates the process by means of a prospective, open, non-interventional patient registry.

Methods: Between March 1st 2016 and July 31 2018 , 35 patients with deep dermal and full thickness burns were included. Primary endpoint is the assessment of efficacy of eschar removal using NXB. Secondary endpoints are depth and area (\%TBSA) of burns treated with NXB and time to wound closure.

Results: Mean burnt area is $9.37 \%$ TBSA (range 1-36\%). The mean burnt area TBSA and total surface treated with NXB is $6.42 \%$ (range $1-15 \%$ ) and $1215.2 \mathrm{~cm}^{2}$ (range $86-3135 \mathrm{~cm}^{2}$ ) respectively. The total success ratio is $97 \%$ (34/35 subjects): $57.1 \%$ of patients (20/35) had complete (100\%) debridement, 10 patients $(28.5 \%)$ had excellent results $(90-99 \%)$ and another $2(5.7 \%)$ had good efficacy outcome (80$89 \%)$. Only 1 subject $(2.8 \%)$ had a treatment failure ( $<80 \%$ efficacy). Only 3 out of 35 patients $(8.6 \%)$ are not entitled to reimbursement: one of the patients due to treatment failure, one subject because of offlabel use of the product and another one not being a Belgian citizen. $62.6 \%$ of the NXB treated areas healed with conservative therapy and $37.3 \%$ are treated with STSG. The average time between the incident and treatment was 1.8 days (range $0-5$ days) and the mean time to closure is 28.9 days ( range 12-78 days).

Conclusion: The mean time between the burn injury and treatment is significantly shorter than using surgical excision. NXB treatment has a very high success rate of $97 \%$ and additional surgical excision could be avoided in $>95 \%$ of cases. This preserved viable tissue and reduced the need for surgical cover of the wound with STSG with $62.6 \%$ compared to using surgical techniques only.

O26.06 Results of the NexoBrid enzymatic debridement post marketing study

$\underline{\text { Y Shoham }}^{1}$, A Ben Yaakov², K David², E Klinger², L Rosenberg²

${ }^{1}$ Soroka University Medical Center, BEER SHEBA, Israel

${ }^{2}$ MediWound Ltd, YAVNE, Israel

Objectives: To assess the safety and efficacy of the commercial use of NexoBrid enzymatic debridement of deep burns in the first two years after product launch, including the efficacy of risk minimization activities performed to reduce identified risks such as pain and pyrexia.

Methods: An international, multicenter, retrospective data collection study.

Results: The records of 164 burn patients treated with NexoBrid in 14 EU burn centers were included in the study, $74 \%$ male, $26 \%$ female, aged $42.3 \pm 14.6$ years, suffering from burns covering $12.7 \pm 14.4 \%$ TBSA. The median time from injury to NexoBrid treatment was 2 days. A total of 313 wounds were treated with NexoBrid, 1.9 per patient, each wound covering $4.1 \pm 5.5 \%$ TBSA. The average efficacy of debridement was $93.9 \pm 15.7 \%$ (median $100 \%$ ) after an average treatment duration of $204 \pm 87$ minutes (median 241 minutes). Only 1 patient $(0.6 \%)$ experienced a pain event during the procedure, and only 6 patients (3.7\%) experienced a pyrexia event within 48 hours of the beginning of NexoBrid treatment. After debridement $12 \%$ of the wounds were autografted, $9 \%$ were allografted, and the rest were treated with a variety of products. In addition to the 313 wounds treated with NexoBrid, the patients had 219 additional wounds treated with other standard of care (SOC) means (61\% surgical, $39 \%$ non-surgical). Twelve patients (7.3\%) suffered from wound infection within 7 days of the debridement procedure. The median time to complete wound closure was 39 days for both NexoBrid and other SOC treated wounds. In general, the overall safety profile of NexoBrid treated patients was good, and consistent with the safety data known from previous studies.

Conclusion: The results of this trial demonstrate the safety and efficacy of NexoBrid in commercial use, confirm the effectivity of the risk minimization activities performed, and are in line with former clinical trial results.

O26.07 Feasibility and Safety of Enzymatic Debridement for the Prevention of Surgical Escharotomy in Circumferential Deep Burns at the Distal Upper Extremity

S Fischer, Y Diehm, V Schmidt, J Horter, U Kneser, C Hirche

BG Clinic Ludwigshafen, LUDWIGSHAFEN, Germany 
Objectives: Burn induced compartment syndrome (BICS) is a severe sequel after circumferential burns at the extremities avoidable by immediate pressure release by escharotomy in circumferential burns. While the current gold standard is surgical escharotomy with a considerable morbidity, this study evaluates safety and effectiveness of immediate enzymatic debridement (ED) to prevent from need for surgical escharotomy and thus BICS development.

Methods: From 2015 to 2017, all patients suffering from deep circumferential burns at the upper extremities requiring surgical escharotomy were potential candidates for the treatment algorithm evaluated by this study. Exclusion criteria involved burn trauma $>12 \mathrm{~h}$, clinically evident BICS, intolerance against the enzymatic debriding agent, deep dry burns requiring pre-soaking as well as blast and electrical injuries requiring fasciotomy or carpal tunnel release. All included patients, received ED with Nexobrid immediately after admission to our burn center. ED was applied according to the manufacturer's recommendations. After ED, extremities were revisited for necessity of conversion to conventional surgical escharotomy every 2 hours for 24 hours. Indication for and time-to skin grafting was reviewed and functional outcomes assessed during follow-up examination.

Results: Thirteen patients with 20 burnt upper extremities were included in this study. ED provided a sufficient eschar removal in all patients, thus conversion to conventional surgical escharotomy was not necessary in any patient. Secondary skin grafting was required in 14 patients. Functional outcomes were favorable 11.9 months after burn trauma.

Conclusion: If the specific contraindications are respected, ED is safe and effective after deep circumferential burns at the upper extremity, prevents BICS, this making surgical escharotomy unnecessary

O27.01 Long-term effects of burn injuries on morbidity and mortality: a systematic review

A Pijpe ${ }^{1}$, DD Rijpma1', CVA Van Hövell tot Westerflier², LJ Schoonmade², PPM Van Zuijlen³, E Middelkoop 4

${ }^{1}$ Red Cross Hospital, BEVERWIJK, Nederland

${ }^{2}$ Amsterdam UMC, Vrije Universiteit Amsterdam, AMSTERDAM, Nederland

${ }^{3}$ Red Cross Hospital; Amsterdam UMC, Vrije Universiteit, BEVERWIJK; AMSTERDAM, Nederland

${ }^{4}$ ADBC; Red Cross Hospital; Amsterdam UMC, Vrije Universiteit, BEVERWIJK; AMSTERDAM, Nederland

Objectives: Advances in burn management have shifted the focus of burn care and research from survival to survivorship. In recent years, management of scars, physical functioning and quality of life has gained increasing attention. In addition, burn survivors may be at increased risk of certain diseases and health outcomes, like cardiovascular disease and cancer. Biological plausibility for such effects comes from evidence that burns cause a significant systemic, inflammatory, and metabolic response which can persist for years. The aim of this systematic review was to investigate what is currently known about long-term effects of burn injuries on morbidity and mortality.

Methods: We searched PubMed, EMBASE and Cochrane Library until July 19th 2018. Search terms included burns, postburn, long-term, adverse effect, morbidity, mortality, complication. Studies assessing long-term (i.e. follow-up $\geq 12$ months postburn) incidence of morbidity and mortality were included. We excluded animal studies, case studies, reviews, non-English articles, and studies on scar quality, physical fitness, and quality of life. Screening, data-extraction, and risk of bias assessment were performed by two investigators independently. Data extracted included study and patient population characteristics, outcome category, incidence rates and relative risks. The review protocol was registered at PROSPERO (CRD42017075899); PRISMA statement guidelines were followed.

Results: We included 49 studies $(>140,000$ patients) covering mortality $(n=11)$, cardiovascular $(\mathrm{n}=12)$, metabolic $(\mathrm{n}=6)$, musculoskeletal $(\mathrm{n}=6)$, malignant $(\mathrm{n}=4)$, gastro-intestinal $(\mathrm{n}=2)$, infectious $(\mathrm{n}=2)$, pulmonary $(\mathrm{n}=2)$, renal $(\mathrm{n}=2)$, and neurological $(\mathrm{n}=2)$ diseases. The majority of studies included burns of all causes $(n=44)$ and were retrospective longitudinal cohort studies $(n=33)$ or prospective longitudinal cohort studies $(\mathrm{n}=13)$. Further data-extraction and risk of bias assessment is currently being performed. 
Conclusion: These findings establish the likelihood of the existence of long-term effects of burn injuries on morbidity and mortality. This may have clinical implications including preventive measures and extended targeted follow-up.

O27.02 Health Related Quality of Life is Decreased after Necrotizing Soft-tissue Infections.

ISuijker ${ }^{1}$, A. De Vries ${ }^{1}$, V.M. De Jong², T. Schepers'2, K.J. Ponsen³, J.A. Halm²

${ }^{1}$ Red Cross Hospital, BEVERWIJK, Nederland

${ }^{2}$ Amsterdam UMC, location AMC, AMSTERDAM, Nederland

${ }^{3}$ Medical Center Alkmaar, ALKMAAR, Nederland

Objectives: A Necrotizing Soft Tissue Infection (NSTI) is a rare but severe infection with a high mortality rate of $12-20 \%$. Diagnosing is challenging and often delayed. Treatment consists of surgical debridement of all necrotic tissue and administration of antibiotics. Despite adequate treatment, survivors are often left with extensive wounds, resulting in mutilating scars and functional deficits. Both the disease the subsequent scars can negatively influence the Health Related Quality of Life (HRQoL). The current study was performed in order to contribute to the knowledge about HRQoL in patients after NSTI.

Methods: We retrospectively identified patients treated for an NSTI in a tertiary centre in the Netherlands. Patient and treatment characteristics were collected and patients were asked to fill in a Short Form 36 (SF-36) questionnaire.

Results: Forty-six patients with a diagnosis of NSTI were identified. Twenty-eight (61\%) were male, mean age was 57 years. Thirty-nine patients $(80 \%)$ survived. Thirty-one $(84 \%)$ of the survivors returned the questionnaire after a median follow-up of 4.1 years (IQR 2.4-5.9 years). Statistically significantly decreased scores when compared to the Dutch reference values were observed for the SF36 domains Physical Functioning, Role-Physical functioning, General Health and the combined Physical Component Score. No differences were observed for the other five domains nor for the Mental Component Score.

Conclusion: This study confirms that NSTI negatively affects HRQoL as reported by the patient, especially on the physical domains. To learn more about HRQoL in patients after NSTI, studies in larger groups with a more disease specific questionnaire should be performed.

Picture 1: https://www.eventure-online.com/parthen-uploads/89/9EBA/add_1_540164_3be9f9936478-4e54-b9fe-fd583c0dde56.jpg

O27.03 Evaluation of Enoxaparin dose adjustment in acute burn patients

MA Mokline, BH Ben Ali, EK EL Faleh, HF Fraj, BM Ben Maamer, BSM Ben Saad, TL Thabet, AAM Messadi

Trauma and Burn Center, TUNIS, Tunisia

Objectives: To assess Enoxaparin dose in burns using burn injury size (TBSA) and weight to achieve adequate anti factor Xa levels (anti-Xa) and its impact to reduce venous thromboembolic events (VTE).

Methods: A prospective study was conducted in burn center in Tunis during 12 months. Acute burn patients admitted to the burn center and anticipated to be non ambulatory for greater than 48 hours were included. Were excluded patients with any contraindication to the use of enoxaparin, and those with creatinine clearance $<30 \mathrm{ml} / \mathrm{mn}$ or creat $>1.6 \mathrm{mg} / \mathrm{dl}$. Enrolled patients received Enoxaparin as following: Enoxaparin dose in $\mathrm{mg}$ Q12Hrs $=22.8+(3.3 \times \% \mathrm{TBSA} / 10)+(1.89 \times($ weight in $\mathrm{kg}) / 10)$ [1] Peak anti-Xa was obtained between 3 and 5 hours after the third enoxaparin dose, consistent with timing of the peak steady state level of enoxaparin. Doses of enoxaparin were titrated up or down by $20 \%$ to achieve the recommended anti-Xa of 0.2 to $0.4 \mathrm{U} / \mathrm{ml}$.

Results: 60 burned patients were included. The mean age was 37,97 $\pm 17,5$ years with a ratio sex of 2,33 . The average TBSA was $33 \pm 19,6 \%$ with a body weight of $75,7 \pm 19,87 \mathrm{Kg}$. Twenty six patients $(43 \%)$ reached anti-Xa target initially, 30 patients' anti-Xa was below target and only 4 patients'anti-Xa was above target. The median final enoxaparin dose was 40mg Q12Hrs (range, 30-80mg) for all patients who were at anti-Xa target. No episodes of hemo-rrhage, thrombocytopenia, or heparin-associated 
allergy documented in any of the study. Patients VTE occured in 5 patients among which 4 cases in patients' anti-Xa below target initially.

Conclusion: Enoxaparin dosing using burn size injury and weight allows to reach a prophylactic initial anti-Xa level, and was associated with a low incidence of VTE events and no bleeding complications.

O27.04 Burns centres Outcomes Registry the Netherlands: evaluation of implementation and the first short term results

TM Haanstra ${ }^{1}$, M Van Baar ${ }^{2}$, N Van Loey ${ }^{3}$, E Middelkoop ${ }^{4}$, M Nieuwenhuis ${ }^{5}$, A Pijpe ${ }^{6}$, C Van Schie $^{7}$, S Scholten-Jaegers, ${ }^{8}$ CH Van der Vlies', PPM Van Zuijlen ${ }^{6}$

${ }^{1}$ Dutch Burns Foundation/Association of Dutch Burns Centres, BEVERWIJK, Nederland

${ }^{2}$ Association of Dutch Burns Centres, Maasstad Hospital, ROTTERDAM, Nederland

${ }^{3}$ Association of Dutch Burns Centres and Utrecht University, BEVERWIJK, Nederland

${ }^{4}$ Association of Dutch Burns Centers, Amsterdam UMC, Vrije Universiteit, BEVERWIJK, Nederland

${ }^{5}$ Martini Ziekenhuis and the Association of Dutch Burns Centres, GRONINGEN, Nederland

${ }^{6}$ Burn centre, Red Cross Hospital, BEVERWIJK, Nederland

'Dutch Burns Foundation, BEVERWIJK, Nederland

${ }^{8}$ Martini Ziekenhuis, GRONINGEN, Nederland

${ }^{9}$ Burns center, Maasstad Hospital, Erasmus MC, University Medical Center Rotterdam, ROTTERDAM, Nederland

Objectives: The Burn centres Outcomes Registry the Netherlands (BORN) aims to routinely collect outcomes of care relevant to the patient, and was implemented recently in three Dutch burn centres. Outcomes include (patient reported) measures of functioning, quality of life, scar quality and psychosocial outcomes. A real-time feedback system of the measurement results ensures that, next to using group based data for research and benchmarking purposes, individual results can be used in clinical processes for goal-setting, shared decision making and treatment evaluation.

Implementing such innovations in healthcare is challenging as it requires changes in culture, mindset and behaviour on multiple levels. It is therefore important to evaluate whether BORN works as intended on a process and content level.

Objective is to evaluate the implementation of BORN in three Dutch Burns centres and to present short term (retrospective quality of life; 1 week post-discharge; 3 months post-discharge) outcomes so far.

Methods: Semi-structured interviews will be held with patients and burn care professionals in three burns centres to get insight in the value and use of the BORN real-time feedback system relative to the measurement burden for patient and staff. Interviews will be audio-taped and transcribed, whereafter theme-based coding will be performed. Outcomes and user-data are used to get insight in patterns of inclusion and follow-up rates, descriptive statistics will be used for analyzing outcomes.

Results: 138 patients are included in the BORN registry to date (march 2019); follow-up rates so far are $90 \%$ for measurement $1 ; 77 \%$ for measurement 2 ; and $70 \%$ for measurement 3 . Preliminary process evaluation shows that a.o. staff shortage and IT support are perceived as barriers for implementation. Further results will be available in Sept. 2019.

Conclusion: Evaluation and learnings after 1 year BORN will evolve in the next months and will be presented during EBA congress.

O27.05 Epidemiology, treatment (costs) and long-term outcomes of patients with fireworks-related injuries (ROCKET); a multicenter prospective observational case series

\section{D.T. Van Yperen}

Burn Center Maasstad Hospital, ROTTERDAM, Netherlands

Objectives: The main aim of this study is to provide detailed information about the injury characteristics of patients reporting to a hospital with fireworks-related injuries. Secondary aims are to determine the treatment (and associated direct medical and indirect societal costs), clinical and 
functional outcome.

Methods: This is a prospective multicenter observational case series. All patients (no age limit) with fireworks-related injuries occurred between December 1, 2017 and January 31, 2018 are eligible for inclusion. We obtained data regarding injury characteristics, direct (medical) and indirect (societal) costs, clinical and outcome. Patient follow-up is at 3, 6 and 12 months after trauma.

Results: Out of 63 eligible patients we included 54. Fifty (93\%) were men with a median age of 15 years and twenty-six patients were minors ( $<16$ years old). Half of the patients were bystanders at the moment of trauma. Most injuries were caused by bangers ( $41 \%)$ and small decorative fireworks $(26 \%)$. In 54 patients we noted 79 injuries, mostly located to the upper extremity and eyes. Most common injuries were burns.

Since 12 months follow-up is completed only recently, the data of the long-term effects will be analyzed in the near future and we will present the results at the moment of this presentation.

Conclusion: This study shows that in particular young men have injuries caused by fireworks and that $50 \%$ is a bystander. Most injuries are caused by bangers and small decorative fireworks, mostly resulting into burns to hands or fingers, or eye injuries. The long-term effects of fireworks-related injuries will be presented at the moment of this presentation.

O27.06 Evaluating the adequacy of treatment in transferred burn patients: a national burn center perspective

\section{R.A. Widyana, A Wardhana, T Toar, M Fitriani}

Cipto Mangunkusumo Hospital, JAKARTA, Indonesia

Objectives: With limited number of burn center, Indonesia makes many transfers for burn patients. As stated by EMSB, proper documentation and treatment for these patients lead to better outcome in the receiving unit. However, the actual practice in the field may not always follow the gold standard form the guideline. Discrepancies in diagnosis and treatment are often possible as shown by a prior study.

Methods: We conducted a study in Cipto Mangunkusomo Hospital, Jakarta as the national burn center to evaluate the documentation and management of referred burn cases. Patients admitted within 24 hours post burn were analyzed for primary survey, BSA diagnosis, and rehydration therapy that are considered substantial for patients transfer. Data was obtained from transfer documents and medical records.

Results: From 163 patients in 2017, 67 were eligible. Fire was the main cause in 70\% patients with average TBSA of $30.6 \% .14 \%$ patients were referred with no data and $44.7 \%$ patients had incomplete data. From the available data, primary survey was mostly filled with oxygen prescription in $77 \%$ patients and lacking in temperature in only $44 \%$. BSA diagnosis was correct in $20 \%$, over for $34 \%$, and under in $7 \%$. Rehydration was adequate in only $2.9 \%$ patients, over for $17.9 \%$ and under for $50.7 \%$.

Conclusion: The main problems in burn patients referred to our hospital are incomplete documentation and incorrect management; most were over-diagnosed but under-hydrated; that may worsen the outcome. Further research is needed to solve this problem that may also exist in other developing countries.

\section{O27.07 MANAGEMENT OF ELDERLY BURN PATIENTS: OUR CLINICAL EXPERIENCES}

\section{IS SOZEN ${ }^{1}$, NT TURHAN ${ }^{2}$, ACY YASTI $^{3}$}

${ }^{1}$ Ankara Numune Education and Research Hospital, ANKARA, Turkey

${ }^{2}$ Sancaktepe Education Research Hospital, ISTANBUL, Turkey

${ }^{3}$ Health Sciences University, ANKARA, Turkey

Objectives: Due to advancements in burn care the success of burn treatment has improved, however, mortality rate for elderly patients with similar Total Burn Surface Area is still significantly higher than adult burn patients.

Methods: A retrospective study was conducted on our hospitalized patients between 2011 and 2015. Data were retrieved from patient files and age, sex, TBSA, the place of the burn injury, whether house fire was accompanied or not, burn agent and mortality were recorded. Patients divided into adult 
and elderly groups. The adult group was between 18 and 65 years old. Elderly patients were grouped as 65 years old or older.

Results: Of the 2258 inpatients, 285 (12,6\%) were 65 or over. In elderly patients, female gender was similar to male gender, $50,5 \%$ vs. $49,5 \%$, whereas in adults male patients were more prone to burn injury than females, $73,4 \%$ vs. $26,6 \%$ ( $\mathrm{p}<0.001$ ). Diabetes, cardiac disease and Chronic Obstructive Pulmonary Disease proportion were significantly high in elderly $(\mathrm{p}<0.001)$. Third degree burns were more prevalent in elderly group, $43,9 \%$ vs. $39,4 \%$. Most burn injuries in the elderly occurred at home $(85,8 \%)$ and house fire was accompanied the burn injury in 9,4\% of elderly patients $(p=0.02, p=0.001$, respectively). The leading cause of burn in the elderly group was scalding $43,5 \%(\mathrm{p}<0,001)$. In the adult patient group, fire was significantly more frequent $44,2 \%(p<0,001)$. Baux score for elderly was 89,9 and 54,8 for adult patients $(\mathrm{p}<0,001)$. The elderly group had significantly higher mortality rate, $18,6 \% \mathrm{vs}$. $10,1 \%(\mathrm{p}<0,001)$.

Conclusion: Elderly burn patients still have significantly higher mortality risk. Natural aging process leads to delayed burn wound healing, increased morbidity and mortality. Pre-existing comorbid conditions and impaired inflammatory response are indicators for worse outcome in elderly. As most of the elderly burn injuries are preventable, passive and active prevention strategies should be encouraged.

O28.01 From strategy to execution: Nurses' competence in burn care and critical care nursing in Helsinki Burn Centre and intensive care unit.

\section{H Siivonen}

\section{Helsinki Burn Centre, ESPOO, Finland}

Objectives: Our human resource goal at Helsinki Burn Centre and intensive care unit is highly competent nursing personnel. To achieve and sustain the high level of nursing competence and extensive set of different skills needed in burn care and critical care nursing, an implementation plan, described in this presentation, was created.

Methods: Description of the background and unique features of the new, three-year-old Helsinki burn centre and intensive care unit is used as a method. The description includes statistics between the years 2016-2018 on human resources, organizational- and operational figures, roles and responsibilities, orientation and on-the-job multi-professional specialty programs and an example of nurse's career path.

Results: An extensive implementation plan was created to aim at our strategic goals. The plan includes educational and managerial key features to help us stay focused and evidence based. We influence and improve the efficiency of nurses' learning through hands-on teaching, coaching the daily management of operational functions and management of human resources. Our management is based on knowledge and we utilize feedback from individuals and teams while investing in multiprofessional teamwork and continuous planning.

Conclusion: To achieve a sustainable high level of nursing competence needed to meet the demands in burn care and critical care, we need committed and motivated nursing personnel. The plan includes a standardized but agile orientation program, maintenance training and simulation program including teambuilding skills. In addition, continuous development through teaching, researching and networking as well as organized efforts to improve and optimize the working conditions are of significance.

\section{O28.02nSimulation-based training of Emergency management for Burn nurses}

M Wormmeester ${ }^{1}$, S Molloy'1, M.K. Nieuwenhuis ${ }^{2}$

${ }^{1}$ Martini Ziekenhuis Groningen, GRONINGEN, Netherlands

${ }^{2}$ Martini ziekenhuis Brandwondencentrum, GRONINGEN, Nederland

Objectives: Since 2013, all Burn nurses of the Burn Centre Groningen, The Netherlands, follow annual simulation-based training in order to remain competent during stressful and life-threatening situations in the early management of burn victims. We now present our experiences and analyse the impact of this training.

Methods: Simulations are performed by groups of three, randomly selected, nurses at the centre's 
admission room. A professional casualty simulator plays the role of burn victim. During the simulationbased training, technical and non-technical skills are trained. Since 2016, the 15 principles of Crisis Resource Management (CRM) have been includes in order to train the non-technical skills. CRM goals is to enhance team performances, reduce human error and thus improve patient safety.

Technical skills are evaluated by colleagues and instructors and feedback is given according to an observational checklist. Non-technical skills are evaluated using the video interactive guidance method. With the Ottawa CRM global rating scale (Ottawa GRS) participants assess their level of competence prior to the training.

Results: Approximately 40 nurses participated annually in the simulation-based training. Throughout, nurses showed high competency in their technical skills. Between 2013 and 2017, an increase was found in the average overall performance as assessed by the Ottawa GRS. This increase did not reach significance $(t(77)=-1,0$; one-tailed $p=.16)$. There was a tendency of improvement over time in all other scales (Leadership, Problem Solving, Situational Awareness, Resource utilization, Communication).

Conclusion: First results are encouraging. Going forward, we have recently started using a validated observation scale (Flowerdew et al.) Scoring is conducted by two burn nurses who have completed the European course for simulation instructor. The coming years we will gather more data to evaluate the effect of the simulation based training, including CRM principles on individual participants.

O28.03 First experiences of burn care at home: cooperation between a burn centre and a specialized home care organization

$\underline{\text { S. Cuperus }}^{1}$, K. Timm 1 , J. Bakker 1 , A. De Jong 2

${ }^{1}$ Allerzorg, regio West, WOERDEN, Netherlands

${ }^{2}$ Rode Kruis ziekenhuis, BEVERWIJK, Netherlands

Objectives: Patients with minor burns often visit the outpatient clinic of the burn centre for wound inspection and wound care. These patients have been discharged from a burn centre or have burns that do not meet the criteria for admission. Visits are often accompanied with travel time and costs and patients also report stress.

Methods: In order to reduce the number of outpatient clinic visits, travel time, travel costs and stress, a burn care nurse in training for Nurse Practitioner and working for a home care company that is specialized in wound care, has taken the lead in optimizing the cooperation of the home care company with a burn centre since September 2018. Agreements regarding cooperation were made. Home care nurses received training from the burn centre on a regular base. This training included wound care, psychosocial care and case discussion. Furthermore, nurses working at the specialized district nursing company have been exchanged to increase knowledge

Results: As experienced by the home care nurses, training by experts from the burn centre and collaboration with the burns nurses increased their knowledge level regarding burn wound care and burns nursing. A common procedure and nursing standard based on evidence and practice have been written, adjusted and secured. Agreements and procedures are evaluated every three months.

Conclusion: Future research will focus on the effect of this collaboration and training on the number of outpatient visits, the extent of stress, the level of patient satisfaction and comfort in their familiar environment, the nature of autonomy and self-management of the patient, and earlier discharge from the burn centre.

\section{O28.04 Coordinating EMSB courses in Norway}

D Hovland, LJ Ulveseth, J Skiftesvik, GT Fitjar, RL Brekke, S. Almeland

Haukeland University Hospital, BERGEN, Norway

Objectives: The Norwegian National Burn Center decided to implement EMSB as a national course for burn care in Norway. A key factor in implementing the course was to establish an organizing team and burn center nurses aimed to establish a coordinating team.

Methods: Since 2015 four nurses employed in the National Burn Center, Haukeland University 
Hospital have been trained by Nordic and European colleagues and have had the responsibility of coordinating and organizing the practical implementation of the Norwegian EMSB courses.

Results: The first Norwegian EMSB course took place in March 2015 with guidance from our Finish colleagues and under the surveillance of one of the founders of the course from Australia, resulting in a license to organize Norwegian providers courses. An instructors course was organized in 2016 and two nurses from the coordinating staff qualified as instructors.

During the first four years we have organized six courses with approximately 144 participants raising awareness on how to manage the severe burns that are admitted into Norwegian hospitals. In addition the majority of nurses in The Burn Centre have completed the EMSB course.

Conclusion: Implementation of EMSB courses in Norway has been successful. This is likely due to the establishment a rigorous coordinating team with proper training and a close collaboration with the international EMSB network.

O28.05 Nursing workload classification to ensure patient safety and quality of care: A literature study to learn about tools to support nursing personnel resource management

\section{A.S.U. Schildt}

Helsinki University Hospital, ESPOO, Finland

Objectives: The Helsinki Burn Centre was united in 2016 with a general ICU which induced challenges in management of nurse resources. Currently the tools to assess the patient workload correlates insufficiently to the reality of the patient care in a unit with general ICU patients, burn patient in intensive care and ward care as well as the outpatient clinic. This study aims to improve and optimize the resource management of nursing personnel in a unit with diverse patient material.

Methods: A systematic literature review was performed on existing classification systems and nursing workload assessment tools. The focus was on the application of these tools in nursing personnel management.

Results: A variety of classification and assessment systems are used in hospitals to measure and predict nursing workload and patient care needs. Although widely used, none of the available tools are suitable for general use. In many cases adjustments and developments have been made to existing tools to better fit the purpose of the unit in question. From a nursing personnel managerial point of view, even if some promising projects have been performed, no obvious solution have emerged how to improve the workforce allocation problem at our unit.

Conclusion: There are many things yet to learn about assessment and prediction of nursing workload to enable optimized management of nursing personnel resources in a unit with diverse patient material. The development of a generally valid system would be favourable but seems still not attainable. Concerning the Helsinki Burn Centre and the challenges faced, a local solution needs to be worked out to best suit the needs of the unit. A project to perform this has started.

O28.06 Towards excellent burn nursing by joining forces in Academy of Burn Nurses

S.A. Van Ingen Schenau - Veldman ${ }^{1}$, CG Bijker ${ }^{1}$, J.J. Prudhomme van Rein², S 't Hart', N Trommel', C Van Schie ${ }^{4}$, A De Jong ${ }^{2}$

${ }^{1}$ Martini Hospital, GRONINGEN, Netherlands

${ }^{2}$ Rode Kruis Hospital, BEVERWIJK, Netherlands

${ }^{3}$ Maasstad Hospital, ROTTERDAM, Netherlands

${ }^{4}$ Dutch Burns Foundation, BEVERWIJK, Netherlands

Objectives: In April 2018, the Academy of Burn Nurses was established and launched during the meeting of the Dutch Association for Burn Care. The goals are:

To promote high quality of nursing care for patients with burns;

To increase leadership, professionalization, positioning, cooperation, education, innovation and nursing research;

To establish and maintain mutual, national and international contacts and to promote exchange;

To aim uniformity in daily burn nursing practice.

Methods: The Academy is a platform consisting of six dedicated burn nurses from the three Dutch 
burn centres and a representative from the Dutch Burns Foundation that aims to work together, share knowledge and to further develop the profession. The Academy meets six times per year and discusses the progress of current projects and priorities for new projects. Twice a year preceding the meeting of the Dutch Association for Burn Care, symposia will be organized and all interested burn nurses are invited to participate.

Results: Until now, a large group of delegates from all Dutch burn centres attended two symposia and rated them positively. Included in the symposia were information about the aim of the Academy, current research projects, and a lecture about nursing leadership by the invited chair of the Dutch Chapter of the Nursing Honour Society Sigma Theta Tau International. Besides, the Academy assessed lacks of knowledge and educational needs and developed a website.

Conclusion: Since nurses indicated that the Academy has a surplus value, future efforts will be made in order to reach the aims and to further develop the profession.

O28.07 An investment in knowledge pays the best interest! (Benjamin Franklin)

\section{A Mekkering}

Martini Hospital, GRONINGEN, Netherlands

Objectives: Since 2008, general nurses from the three Dutch Burn Centres receive the specialized continuation education for burn care nursing (EBN). The aim of the EBN is to equip nurses to safely deliver highly complex burn care in a multidisciplinary team, in which nurses participate as independent professionals.

Methods: To be able to deliver this high quality nursing care, the Burn Centres, in collaboration with the Dutch Burns Foundation, financed some of their own nurses to become professional teachers. Thus, the quality of education can grow, and comply with the Dutch quality cycle. The cycle consists of:

Intensive collaboration between education specialists, scientists, management and professionals of the burn centres.

The professional profile for the burn nurse, based on the CanMEDS competences (English translation available).

Adaptation of the CanMEDS competences to the final objectives of the EBN

Linking attainment to study goals

Examination of both theory and practice

Qualified work tutors, equipped to coach objectively and judge practice tests.

Results: Working according to the quality cycle has resulted in:

Six modules covering various themes: basic competences including clinical reasoning, evidence based practice and learn-to-learn, wound treatment and wound care, intensive care, the paediatric burn patient, psychiatry and psychology. These themes are taught by professionals working at the burn centres. Knowledge therefore remains up to date.

An examination cycle, with both summative as well as formative tests in theory and practice.

Approximately sixteen months of clinical practice learning.

Conclusion: The EBN has developed into a full-fledged continuation education. Its content is eligible for national accreditation from the College of Care Educations and meets the Dutch quality standards. This accreditation is important for burn centres to recruit nurses, educate them to a high standard, and keep them.

Picture 1: https://www.eventure-online.com/parthen-uploads/89/9EBA/add_1_539509_d2bc13e434b7-40c0-b492-178c9eee2215.PNG

\section{O28.08 HUMANISATION OF NURSING CARE: A DAILY CHALLENGE}

\section{Perez del Prado, MJ Sanchez Garcia}

ICS, BARCELONA, Spain

Objectives: Burns patients confront a great traumatic experience that requires a multidisciplinary team, with a holistic strategy to manage such a complex process.

The newly developed wound care techniques and advance biomedical technologies, in burns and 
intensive care units may affect the comprehensive care the patients need. Nurses in our burns unit have been able to integrate this technification with a humanistic approach to guarantee patients' wellbeing.

Methods: We developed a qualitative ethnographic research to collect patients, relatives and staff experiences in Vall d'Hebron burns unit.

Results: Patients and relatives show high satisfaction in nursing care, due to the burns unit humanisation initiatives and nurse's holistic point of view. The most outstanding initiatives that we found were, adapt visit schedule, allow children to visit their relatives or the use of music therapy.

Conclusion: Humanised care increases patient wellbeing and facilitates the recovery in this traumatic experience.

O29.01 Replacement of murine feeder layer with biologically relevant laminin to culture keratinocytes for therapeutic application in burns

AC Chua ${ }^{1}$, MST Tjin², BKT Tan', KT Tryggvason²

${ }^{1}$ Singapore General Hospital, SINGAPORE, Singapore

${ }^{2}$ Duke-NUS Medical School, SINGAPORE, Singapore

Objectives: The current expansion of autologous human keratinocytes (HKs) to resurface severe and extensive burns still relies on murine feeder layer (3T3-J2) and calf serum in the cell culture system for optimal growth and stem cell maintenance. Despite their clinical efficacy to treat severe burns, the presence of undefined xenogeneic components in this culture system still poses some safety concern in today's regulatory framework. In vivo organized cell types such as epithelial cells are tightly anchored to a basement membrane (BM). Among many of the BM components, laminins are the only components that are highly cell type specific and contribute to the BM structure and behavior of associated cells. We hypothesize that the use of biologically relevant laminin matrices could be used to replace feeder and support the growth of HKs in vitro.

Methods: Through our characterization efforts of the human skin basement membrane and 3T3$\mathrm{J} 2$, we found two laminin isoforms could best support adult HK growth in a completely xeno-free and chemically defined method. We validated keratinocytes cultured on these two laminin systems vs. the standard 3T3 co-culture method by assessing their growth potential, colony forming efficiency, expression of basal and differentiation markers by qPCR, immunostaining, FACS as well as organotypic culture functional assay and grafting using a flap model on nude mice.

Results: We demonstrate that our laminin system is comparable to the 3T3-J2 co-culture system in terms of the cultured keratinocyte basal and differentiation expression profile, cell growth, colonyforming efficiency and the ability to form normal stratified epidermal structure in both in vitro and in vivo models.

Conclusion: As our culture system is xeno-free and fully defined, this method will not only provide safer products for the patient, but also open up greater applicability in epithelial cell therapies for less severe burns and chronic wounds.

O29.02 Simultaneous application of Glyaderm $\mathbb{R}$ as a dermal substitute with autologous split skin grafts in the reconstruction of deep burns and/or full thickness defects: preliminary results.

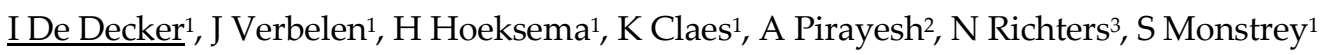

${ }^{1}$ Ghent University, GHENT, Belgium

${ }^{2}$ Amsterdam Plastic Surgery, AMSTERDAM, Nederland

${ }^{3}$ Euro Tissue Bank, BEVERWIJK, Nederland

Objectives: Absence of the reticular dermal layer is inherent to the use of autologous STSG, often resulting in hypertrophic scars and contractures. Several dermal substitutes have been developed, unfortunately with variable results in terms of improvement compared to the use of STSG alone. Bilayered skin reconstruction using a "human derived dermal substitute" (Glycerolised Acellular Dermis (Glyaderm $\left.{ }^{\circledR}\right)$ ) has already been proven to result in significantly improved scar quality using a two-step procedure. Our current study will evaluate whether the simultaneous application of Glyaderm ${ }^{\circledR}$ and STSG will result in superior scar quality.

Methods: A prospective, controlled, randomized, intra-individual, single-blinded study is 
performed, investigating the simultaneous application of Glyaderm ${ }^{\circledR}$ and STSG versus STSG alone in full thickness burns or comparable skin defects in 75 patients. Scars are evaluated during the acute phase and at 3, 6, 9 and 12 months. Subjective and objective measurement systems are used. Relevant outcome parameters such as pain, elasticity, colour, itch, rigidity, trans epidermal water loss are evaluated. Biopsies for histological analysis are taken at 3 and 12 months follow-up.

Results: 49 patients are included so far, resulting in 59 wound comparisons: 31 full thickness burns, 21 phalloplasties and 7 other full thickness skin defects. Graft take was optimal in both the Glyaderm ${ }^{\circledR}$ group as the control group (95.86\% and $96.70 \%$ ) Pain and time to wound healing were comparable in both groups. Differences were found in terms of colour, pigmentation and pliability in favour of Glyaderm ${ }^{\circledR}$ and STSG. At every follow-up, the majority of the patients favoured Glyaderm ${ }^{\circledR}$ in terms of scar quality during a visual evaluation.

Conclusion: Preliminary results of the simultaneous application of Glyaderm ${ }^{\circledR}$ and STSG provided satisfactory outcomes in terms of pain, time to wound healing, graft take, colour, pigmentation, pliability and visual evaluation. The early results of other parameters i.e. elasticity provided interesting outcomes, though not significant.

O29.03 3D bio-printing for the treatment of acute burns wound - in vitro and in vivo development

\section{$\underline{\text { S. BROSSET }}{ }^{1}$, A. DESANLIS ${ }^{1}$, A. THEPOT ${ }^{2}$, C. AUXENFANS ${ }^{1}$, C. MARQUETTE ${ }^{3}$, M. ALBOUY ${ }^{2}$,}

\section{F. BRAYE ${ }^{1}$}

${ }^{1}$ Edouard Herriot Hospital, LYON, France

${ }^{2}$ Labskin creation, LYON, France

${ }^{3} 3 \mathrm{dFab}, \mathrm{LYON}$, France

Objectives: The aim of this study is the development of a novel per operative procedure of dermo epidermal graft engineering and the proof-of-concept validation of a mobile skin 3D bioprinting system that could be used in the management of acute burns wound.

Methods: The technique used is based on the adaptation of a 3D skin bioprinting technique used to create dermo-epidermal grafts for cosmetology studies. A medical grade bio ink composed of fibrinogen, alginate and gelatin is used as a matrix. The used cells, keratinocyte and fibroblast were obtained by mechanical extraction from human skin biopsies. The printing is performed directly on the wound using a robotic arm.

Results: The new formulation of medical bio-ink allows the in-vitro printing of a lively and functional dermis and epidermis. These regenerated tissues have a dermal structure and composition similar to healthy skin, with neosynthesized elastin fibers and extensive collagen deposition arranged in large organized fibers allowing the development of a proliferative and differentiated epidermis. This cellular structure proved a good in-vivo tolerance for mice.

Conclusion: 3D bioprinting of skin substitutes is a promising tool for the management of very extensive acute burns. The results obtained assume that the intraoperative in-situ production of autologous dermo-epidermal constructs may be possible in the future.

O29.04 Wound healing after cultured epithelial autografting in patients with massive burn injury: a cohort study

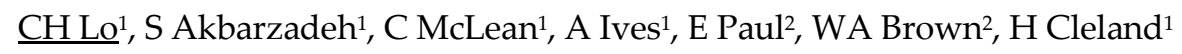

${ }^{1}$ The Alfred, MELBOURNE, Australia

${ }^{2}$ Monash University, MELBOURNE, Australia

Objectives: Last century, our laboratory produced Cultured Epithelial Autograft (CEA) for clinical use by the affiliated adult burn service and other burn units across the country. Production of CEA for clinical use was discontinued after several years because of a low success rate and subsequent low demand. The aim of this study was to re-examine the use of CEA in our unit since the introduction of immediate burn wound excision and use of a synthetic skin substitute to temporize the excised burn wound.

Methods: This observational cohort study recruited patients with burns exceeding 35\% TBSA admitted to the Victorian Adult Burns Service at The Alfred (December 2013 - December 2016). 
Autologous keratinocytes were expanded and delivered via sheets of fibrin carrier.

Results: Twelve patients were recruited to participate in the study. Thirty-two sites were treated with CEA. CEA applied in combination with widely meshed SSG led to the highest take rate $(90.1 \%)$ at 7-10 days. Further debridement and grafting was necessary in sixteen of thirty-two sites treated, all involving wound beds prepared with Cuono method or sites treated with CEA only.

Conclusion: It is important to address the problem of wound bed contamination, either through increased resistance on the part of the construct or wound bed sterilization. Improved understanding of the relative importance of vascularisation, control of cell behaviour, the extracellular matrix, immune function and intrinsic antimicrobial capacity for graft take would then inform a more targeted approach to skin tissue engineering for wound closure in severe burns.

O29.05 A systematic review: current trends and take rates of Cultured Epithelial Autografts (CEA) in the treatment of patients with burn injuries

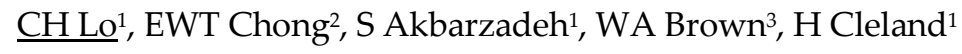

${ }^{1}$ The Alfred, MELBOURNE, Australia

${ }^{2}$ Royal Victorian Eye \& Ear Hospital, MELBOURNE, Australia

${ }^{3}$ Monash University, MELBOURNE, Australia

Objectives: In the clinical translation and application of Cultured Epithelial Autografts (CEA), several issues persist. The aim of this systematic review is to determine (1) current practice and trends in clinical application of CEA and (2) clinical efficacy of CEA.

Methods: A structured literature search was performed in Ovid MEDLINE from 1946 and Ovid EMBASE from 1974 till present. All published peer-reviewed randomized or non-randomised clinical studies, cohort studies, prospective or retrospective series involving human application of CEA in the setting of burn injury were included.

Results: From 7267 studies initially identified, 77 studies were included in the analysis. Ninety-six percent (74/77) of these series had a sample size of less than 100 patients. In 76.6\% (59/77) publications, average burn treated exceeded $40 \%$ TBSA. Overall, CEA take rates reported in the literature were inconsistent and varied significantly from $0-100 \%$. There was a recent trend for co-application of CEA with autologous graft, achieving relatively high and consistent take rates of 73-96\%.

Conclusion: Results from CEA application remained unpredictable. CEA remains an adjunct or biological dressing, and not an alternative to conventional SSG. However, CEA has contributed to wound closure and it has been life saving in selected circumstances. Skin tissue engineering should continue as the clinical need for skin replacement is foreseeable into the future.

O29.06 Wood-derived nanofibrillar cellulose dressing as a cell scaffold for wound treatment

I. Kiiskinen $^{1}$, A. Merivaara ${ }^{1}$, T. Hakkarainen ${ }^{1}$, S. Miettinen ${ }^{2}$, M. Yliperttula ${ }^{1}$, R. Koivuniemi ${ }^{1}$

${ }^{1}$ University of Helsinki, HELSINKI, Finland

${ }^{2}$ Tampere University, TAMPERE, Finland

Objectives: In the field of regenerative medicine, stem cell transplantation has shown great promise to promote wound healing. In the wound, stem cells could regulate inflammation, and enhance tissue homeostasis and regeneration by secreting multiple growth factors and cytokines. However, a hostile environment of the injured tissue has shown considerably to limit the survival rate of the transplanted stem cells. To improve the cell survival and retention towards successful cell therapy, an optimal cell scaffold is required. In this project, a wood-derived nanofibrillar cellulose (NFC) wound dressing previously studied in patients ${ }^{1}$ is used as a cell culturing platform for multipotent human adipose-derived mesenchymal stem/stromal cells (hASCs) in order to develop a cell transplantation method for wound care.

Methods: hASCs were cultured on top of NFC wound dressing and control cells on tissue culture plastic. Cell viability was evaluated using mitochondrial activity and released lactate dehydrogenase. Cell morphology was analyzed using scanning electron microscopy. Cytoskeletal structure, mesenchymal cell marker expression and proliferation capability were analyzed by immunocytochemical staining and/or by quantitative PCR. 
Results: With the optimal cell density, hASCs cultured on top of NFC dressing maintain cell survival for two weeks without any cell adhesion coatings. In addition, NFC wound dressing does not induce any remarkable cytotoxicity or alter the morphology, the mesenchymal cell marker expression or proliferation capability of hASCs compared with the control cells.

Conclusion: NFC dressing is an efficient culture platform for hASCs without any cell adhesion coatings, and thus, offers a potential method for cell therapy in wound healing treatment in the future.

Picture 1: https://www.eventure-online.com/parthen-uploads/89/9EBA/add_1_540050_ce786cf7caca-407d-9e09-5efe80408ba0.png

Picture 2: https://www.eventure-online.com/parthen-uploads/89/9EBA/add_540050_ce786cf7caca-407d-9e09-5efe80408ba0.png

\section{O30.01 EVALUATION OF THE INTERVENTIONS OF A CLINICAL PHARMACIST IN ADDITION TO TEAM-BASED CARE IN A BURNS UNIT}

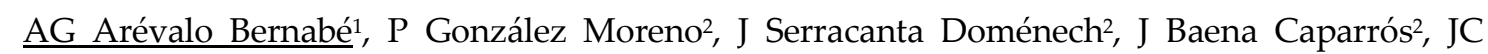
Giménez Juárez², MQ Gorgas Torner², P Lalueza Broto²

${ }^{1}$ Hospital Universitari Vall d'Hebron, BARCELONA, Spain

${ }^{2}$ Hospital Universitari Vall d' Hebron, BARCELONA, Spain

Objectives: The burn patient's heterogeneity makes the managing of these patients complex and they need multidisciplinary solutions. The aim of this study is to evaluate the pharmacological interventions of a clinical pharmacist, as a component of team-based care in a burns unit.

Methods: Prospective observational study with the burns patients admitted in a burns unit from October 2018 to March 2019. A protocol was devised that standardizes and systematizes the review of the pharmacotherapeutic plan of all patients in the burns unit. Also, there is included a model of record and codificate of pharmaceutical interventions. The pharmaceutical interventions registered during the study period are analyzed.

Results: A hundred patients were monitored, of which 56 were men. 23 patients were below 18 years old (group A), 56 were between 18 and 64 years old (group B) and 21 were 65 or older (group C). Mean Charlson Index was 0.086, 0.51 and 4.19, respectively. The mean days of admission were 21.19 (1 -84 ) and there was one death during study period. A total of 76 interventions were proposed and all of them were accepted. Intervention per patient ratio was 0.35 in A, 0.63 in B and 1.24 in C. Pharmaceutical interventions were divided in 10 types, being the most frequent change in route of administration in group A and B (37.5\% and $38.1 \%$, respectively) and treatment suspension $(46.1 \%)$ in group C. Change of dose intervention, considered of high clinical impact, supposed the $9.2 \%$ of interventions in the total of patients.

Conclusion: More interventions per patient are done in over 65 years old patients, who in turn are the patients with the highest associated comorbidity.

These results confirm that a pharmacist in a burns unit can improve the care provided to patients monitoring the drugs administered and identifying preventable adverse drug events, drug interactions and errors.

\section{O30.02 The New National Burn Center of Finland, Department U2}

TM Varpula, L Lindahl, M Hult, T Oksanen

Helsinki University Hospital, ESPOO, Finland

Objectives: To describe organization, patient material and patient outcomes of the new national burn center of Finland

Methods: The National Burn Centre of Finland moved from Töölö hospital to a new facility in Jorvi hospital on January 2016. In the new facility there are 12 ICU beds, 6 burn ward beds and outpatient clinic dedicated for burn patients. Case-mix of the ICU patients of this new facility consist from adult severe burn patients from whole Finland (population of 5 million) and mixed adult ICU patients from western part of Helsinki capital area (population of 350 000). ICU has 24/7 on-call intensivist and it follows closed format ICU organization. ICU takes part in a national ICU quality registry and benchmarking project. A new facility share all resources between different patient groups. 
Results: During the first three years (2016-2018) there were 1043 admissions into ICU of the U2. Diagnostic case-mix is presented in figure 1. These admission comprised from 975 patients, of which $173(17 \%)$ were burn patients. All severe burn patients needing ICU were treated in U2. The main demographics and outcomes of mixed patient population and the burn patients are described in Figure 2. Based on ICU quality registry the standardized mortality ratio was lower than expected $(0,93)$. Outcome of severe burn patients is comparable of the reported in developed countries.

Conclusion: Providing ICU care for burn and mixed ICU patients together in the same facility is feasible and seems to be associated to good outcome for both general and burn ICU patients. At the organizational level there are advantages in the resource allocation and consumption.

Picture 1: https://www.eventure-online.com/parthen-uploads/89/9EBA/add_1_540257_06d49dea08cc-465e-a221-2ba5b3e836ff.JPG

Picture 2: https://www.eventure-online.com/parthen-uploads/89/9EBA/add_540257_06d49dea08cc-465e-a221-2ba5b3e836ff.JPG

O30.03 Increasing burn centre referrals but decreasing burn size: National burn centre trends over 35-years

\section{H Onarheim, AB Guttormsen, S. Almeland, RL Brekke}

Haukeland University Hospital, BERGEN, Norway

Objectives: Since 1984 the Burn Centre at Haukeland University Hospital in Bergen has a national function for major burns in Norway (present population: 5,3 million). This study has addressed changes in admissions and hospital days for burns as well as burn sizes over the recent 35-years.

Methods: The Burn Centre's Quality Registry was searched for all burns admitted from September 1984 through December 2018. Information on area injured, age, LOS, outcome and county of residence was retrieved, stored in Excel 2010, and further analysed using Graphpad PRISM (version 8.0.1).

Results: During the first 35 years of service 2637 patients had been admitted for burns. 1799 patients $(68,2 \%)$ were males. In the most recent 5-year period (2014-18) the average annual number of admissions was doubled compared to in the years 1984-2003 (Table); in the same period the Norwegian population merely increased by $26 \%$. Likewise, in 2014-18 the number of hospital days for burns was $70 \%$ higher than in 1984-2003. With time there was also a clear trend towards a lower median age and smaller mean and median burn sizes (Table). In 2018, $82 \%$ of all hospital days were related to patients transferred from other hospitals.

Conclusion: Particularly in the recent five years more burn patients have been transferred to the Burn Centre, putting a stress on its physical capacity for admitting patients. A declining median area of injury and a lower median age probably partly reflects an increased request to transfer paediatric burn patients even with more moderate burns. In addition to population growth and immigration future planning must take into account such trends towards increased expectations for highly specialized burn care.

Picture 1: https://www.eventure-online.com/parthen-uploads/89/9EBA/add_1_539332_acdb461a79e0-4638-b0d9-c1f7b9507873.jpg

Caption 1: Changes in annual admissions, mortality, hospital days, age and burn size for 5-year intervals

O30.04 Interdisciplinary follow-up care for burn patient

CP Pasquale, C.R. Rosch, SS Simone, JP Plock

University hospital zurich, ZÜRICH, Switzerland

Objectives: Follow-up care of burn patients is demanding and only performed by two specialised centres in Switzerland. The experience of GPs and outpatient clinics in the aftercare of burn patients is limited. Therefore the Zurich Burn Center at the University Hospital Zurich (USZ) has developed a concept specifically designed for the needs of discharged burn patients by providing individualised offsite consultation via telephone or email and dedicated interdisciplinary follow-up sessions in the outpatient clinic by experienced healthcare personnel.

Methods: Monthly follow-up consultation of 2 hours duration per patient $(45 \mathrm{~min}$ advanced 
practice nurse/APN, 45min physiotherapist, 15min consultant plastic surgeon). The final $15 \mathrm{~min}$ consultation by APN, physiotherapist and surgical team member concludes the follow-up and results in a written assessment and treatment plan for the patient.

Results: Patients and outside health care professionals have direct contact for questions related to follow-up care. Patients receive continuous care and education about therapeutic options. The inhospital burn team receives direct feedback from patients and health care professionals about the follow-up. The concept supports and encourages interdisciplinary teamwork among health care professionals. A Periodic medical education sessions were implemented to allow for professional exchange. The concept supports building a network of interested health care professionals outside specialised centres.

Conclusion: The interdisciplinary outpatient clinic has improved the frequency and overall quality of follow-up care of burn patients. Patients have demonstrated initiative and better self-management at home also by offsite communication with the interdisciplinary team via telephone or email. The feedback loop allowed the interdisciplinary team to monitor the patients compliance with treatment recommendations and improve adherence. In summary, the network building effect of the interdisciplinary outpatient clinic has improved the follow-up care of burn patients

O30.05 Consolidation of the 2 Burn Centers of Lyon, France: results at 18 months

\section{FM BRAYE, S. BROSSET, B FARNY, M BERTIN MAGHIT, T RIMMELE}

Hospices Civils de Lyon, LYON, France

Objectives: Our Regional Agency for Health decided to consolidate the Two Burn Centers of Lyon into Hospices Civils de Lyon. It results in a 30-bed Burn Centerwith10 resuscitation beds, 10 ICU / pediatric beds, and 10 surgery beds for buns under $20 \%$ TBSA.

We are located near the new Trauma Center. The Tissue bank is included into the Burn Center. It manages cadaver skin and cultivates skin substitutes for clinical use.

The mean of this study is to evaluate our results in term of survival and to analyze the difficulties of functioning.

Methods: The statistical data were provided by the Informatics Department of the Hospital. The operating difficulties were identified through meeting minutes.

Results: From June 2017 to December 2018 (18 months) we admitted 1171 in-bed patients for acute burns. 238 into resuscitation Unit, 513 inti ICU /pediatrics, and 661 into surgery beds. $27 \%$ were children under 15.

Among burn patients, 1037 suffered burns undes 20\% TBSA, 114 between 20 and 50\%, and 20 over $50 \%$ TBSA.

18 burned patients died, which represents $1,2 \%$ of total admissions. Death is highly correlated to the age (average 81 yeras, range 48-96., rather than to the surface.

Functioning problems are:

- Transmission of the informations

- Instability of the surgeons team.

- Difficulty for the recruitment of rehabilitative doctors

- Difficulty of adaptation to the variations of activity

- Increased dependance to the availability of beds in rehabilitation centers

Conclusion: During the works for renovation and expansion of the Burn center, we decreased our number of beds from 21 to 16 . When starting with 30 beds, we realized that our former organization was obsolete. The quality of care is now maintained at the price of constants efforts of the whole team. We have to adapt our procedures to this new volume of work and information.

O30.06 Streamlining the OR-process of ICU patient with Lean method

\section{Kinnunen}

Helsinki University Hospital Jorvi hospital, HUS, Finland

Objectives: Several challenges have emerged in the OR process of ICU burn patients during preand intraoperative phases. The preoperative preparations have been insufficient for the operation to 
start on time, task distribution was unclear, under- and over resourcing occured and there was lack of cooperation between the OR and ICU. The objective was to improve the efficiency of ICU patient's OR process and to optimize time and human resource use.

Methods: The streamlinig process was made using Lean method. The project group involved was multi-professional and included representatives from ICU, OR and Infection unit. All members had the possibility to share their view of the process and practices. The group achieved a common view of the process during the project meetings and they updated the existing instructions and made new guidelines to improve the process.

Results: After the change, patients entered the OR earlier and OR overtime was reduced significantly $(47 \%)$. Cooperation of the ICU and OR improved and became smoother. Overall, the streamlining significantly influenced on the care of the patient. As the preoperative preparations were sufficient, patients entered the OR on time and the intraoperative phase was shortened. Also, patients' temperature was better maintained enabling the surgery to be completed in one session.

Conclusion: With the help of the Lean method, the OR process was streamlined. Owing to the motivation and commitment of the group to openly discuss and pinpoin defects in the original process and to work together to improve them, the project was successful. Improved multi-professional cooperation became natural and refined to include educational aspects as well. Task distribution will be adjusted further in the future. This will improve the orientation of new personnel and simulation of the operational functions.

O31.01 Complications in tissue expansion: A logistic regression analysis for risk factors.

C Smolle ${ }^{1}$, P Wurzer ${ }^{1}$, SM Spendel ${ }^{2}$, S Spendel ${ }^{1}$, M Schintler ${ }^{1}$, H Friedl2 ${ }^{2}$ LP Kamolz ${ }^{1}$

${ }^{1}$ Medical University of Graz, GRAZ, Austria

${ }^{2}$ Graz University of Technology, GRAZ, Austria

Objectives: Tissue expansion is a popular technique for skin resurfacing, especially after burns. Unlike free flaps or skin grafting, it provides optimal tissue coverage on the one hand and low donor site morbidity on the other. Complication rates are however high, approximating 50\% according to current literature. The aim of this study was to define severity of complications in our cohort and to identify risk factors for complications.

Methods: We retrospectively analysed all tissue expansion procedures carried out at the University Hospital of Graz, Austria between Jan 1st, 2005 and Dec 315t, 2014. Collected data included age, gender, body mass index (BMI), smoking habits, prevalence of diabetes mellitus and mean arterial pressure (MAP). Expander size $(\mathrm{ml})$, expander shape, expansion site, indication (postburn, posttraumatic, congenital deformity), filling mechanism (external/internal valve, osmotic) and number of expanders implanted at a time were assessed. Complications were documented. Risk factors for complications were determined using binary logistic regression analysis.

Results: In total 71 procedures $(37 / 52 \%$ in men and $34 / 48 \%$ in women) were analysed. Complications occurred in $26(37 \%)$ cases. The binary logistic regression analysis resulted in a model where female gender, high expander volume and expansion site in the limbs (compared to head) where significant risk factors for complications $(\mathrm{p}=0.024)$.

Conclusion: Our study revealed that female gender, high expander volume and expansion in the limbs go along with an increased risk for complications. The impact of gender may be due to the wellknown different tissue properties in women when compared to men regarding skin laxity. Still, tissue expansion remains the tool of choice for certain indications such as reconstruction of the hear-bearing scalp. Importantly, tissue expansion after burn injuries does not bear a higher risk for complications.

O31.02 Evaluation of the effectiveness of reconstructive surgery for severe burn scar contractures in sub-Sahara Africa

T.C.C. Hendriks ${ }^{1}$, M. Botman ${ }^{1}$, M. Nieuwenhuis' ${ }^{2}$, P. Van Zuijlen ${ }^{3}$

${ }^{1}$ Amsterdam University Medical Center, AMSTERDAM, Nederland

${ }^{2}$ Burn Center Martini Ziekenhuis, GRONINGEN, Nederland

${ }^{3}$ RKZ, BEVERWIJK, Nederland 
Objectives: Scar contractures are still a major cause for concern and reason for reconstructive surgery, in high as well as low-income countries. Although contracture release surgery is frequently applied to improve function and quality of life (QoL), there is scarcity of well-designed studies, assessing long-term surgical outcomes. This study therefore evaluates the effectiveness of such surgery for severe burn scar contractures, using validated outcome measures.

Methods: This prospective, observational cohort study was conducted in a rural, referral and teaching hospital in Tanzania, sub-Sahara Africa. Eligible were all patients undergoing contracture release surgery between September 2017 and November 2019. Techniques of contracture release surgery (type of grafts, flaps) were documented. Effectiveness is calculated as the difference in range of motion (ROM) and QoL before surgery and at follow-up. ROM and QoL were assessed using validated measures; goniometry, EQ-5D and WHO DAS 2.0 respectively. Follow-up intervals were discharge, 1, 3,6 and 12 months postoperative.

Results: In total 69 joints of 40 patients were operated on, the majority in the upper extremity. Follow-up rate at 6 months was $85 \%$ (34 out of 40 patients). Preliminary results show that in 25 out of 60 joints (42\%), normal range of motion (according to AAOS) was achieved at 6 months follow-up. Mean WHO DAS score difference was $17 \%$ (pre- $22 \%$ (range $0-58 \%$ ) vs. postoperative $5 \%$ (range 0 $17 \%)$ ). EQ-5D score difference was 2 (pre- 7.78 (range 5-12) vs. postoperative 5.78 (range 5-8)). The overall complication rate was $47.5 \%$. Average graft and/or flap take was $90 \%$. Statistical analyses are currently conducted.

Conclusion: This is the first study to evaluate the long-term effectiveness of contracture release surgery. Findings show that the ROM and quality of life improved, and in a vast amount of patient normal range of motion was achieved. Additional data and analyses will be presented.

O31.03 Cultured Keratinocytes (Epifast $\mathbb{R}$ ) and V.A.C. therapy, our first choice for skin graft dressing in chronic wounds burn related.

\section{IB Ruiz-Padilla}

Hospital H+, SAN MIGUEL DE ALLENDE, Mexico

Objectives: The objective of this work is to demonstrate the superior results when a graft is applied with Epifast ${ }^{\circledR}$ as primary dressing and V.A.C as secondary one, compares with "traditional" method.

Methods: A prospective study was carried out with the application of Epifast ${ }^{\circledR}$ and V.A.C. and compared with the results of patients with similar wounds, who had been grafted and covered with organdy (traditional).

Results: Were included 15 pediatric burned patients. A 99\% integration was obtained in the grafted patients to whom Epifast ${ }^{\circledR}$ and V.A.C. therapy were applied, against $60 \%$ of those to whom organdy was applied as a primary dressing. It was not necessary to regraft any of the first group; in the second group, 30\% was regrafted, and the remaining $5 \%$ closed by second intention.

Conclusion: A fundamental part of the complete rehabilitation of the burned patient is the skin coverage. The ideal procedure is the early division among those lesions that will epithelize, and those that will have to be grafted. In severe patients or with comorbidities, it cannot always be done, and the wounds become chronic, making the grafts integration a more complicated problem. Undoubtedly, the combination of Epifast ${ }^{\circledR}$ with V.A.C., it is an alternative that offers superior results compared to the traditional cover of the grafts, that is the reasons the Epifast ${ }^{\circledR}$ plus V.A.C is our first choice dressing in this clinical scenario.

Picture 1: https://www.eventure-online.com/parthen-uploads/89/9EBA/add_1_539747_467fffd96551-4176-bf80-da1479f53601.png

O31.04 The use of free flap for fat and lower limb amputation in case of severe purpura fulminates

FD Duteille, PP Perrot, RN Le floch

\section{CHU Nantes, NANTES, France}

Objectives: Purpura fulminate remains a severe and potentially life-threatening disorder, despite advances in intensive care that have led to a significant increase in the survival rate. One major risk is amputation of extremities. 
Methods: The present report concerns a series of five patients (all male; mean age, 19 years) whose lower limb extremities were salvaged by the use of free flaps in the feet. In total, six flaps were performed (mean per patient, 1.7): four latissimus dorsi, two serratus anterior, and one combination latissimus dorsi/serratus anterior.

Arteriography and end to end anastomosis were always realized. Split thickness was realized 5 to 7 days after the flap when the surgery was considered as a success.

Results: There were no flap failures, and the results are favorable after a mean follow-up of 25.5 months (range, 18 to 34 months), with all patients able to walk normally.

Conclusion: Although microsurgery of this type has been criticized when performed in cases of purpura fulminans, the authors' series indicates that it can be particularly effective and avoid foot or lower limb amutation in youg ptaient

O31.05 ‘Give Me 5 Foundation': A Plastic Surgery Charity Mission Helping to Enhance the Management of Burn Injuries in Rural India

BW Wood ${ }^{1}$, F Nicoli ${ }^{2}$, R Chilgar ${ }^{3}$, H Chen ${ }^{4}$

1Pinderfields Hospital, Wakefield, West Yorkshire, LEEDS, United Kingdom

${ }^{2}$ Royal Victoria Hospital, NEWCASTLE UPON TYNE, United Kingdom

${ }^{3}$ Elrevo Clinic, MUMBAI, India

${ }^{4}$ China Medical University Hospital, TAIWAN, Taiwan

Objectives: Traditionally, the field of Public Health has centred around preventing communicable diseases. Despite this $32 \%$ of the world's disease burden remains amenable to surgical intervention. The Lancet commission has set a target of $80 \%$ coverage of safe surgery and anaesthesia access by 2030 . The WHO reports 260,000 deaths annually secondary to burns with over $50 \%$ occuring in the developing world. In India there are over 7 million burn injuries annually with 100,000 deaths and over 250,000 people living with disability. 'Give Me 5 Foundation' is a no-profit organisation aiming raise awareness and to improve the surgical management in low resource countries; focussing on limb defects secondary to burns, congenital deformities, trauma and infection.

Methods: Based in Shirdi Hospital, Maharasthtra, India between 19th and 25th March 2018 a team of worldwide plastic surgeons worked in tandem with

local healthcare staff to improve the form and function of patients with limb deformities. A diagonal approach was utilised working alongise local healthcare staff, physiotherapists, dressings clinic and public health departments to ensure a sustainable model of care.

Results: Eighty-Eight (88) patients were treated over 4 days. The majority (55) were male with an average age of 24 years. $36 \%$ of cases involved release of post burn skin contractures with the majority involving the neck and axilla (72\%). The remaining procedures included microsurgical reconstruction of traumatic brachial plexus injuries, lymph node transfer for lower limb lymphoedema secondary to filariasis as well as syndactyly reconstruction. At 6 months follow up we have had no complications to date with an average hospital stay of 2 days.

Conclusion: Burns are the leading cause of morbidity in India impacting on the patient's physical and psychological health. Give Me 5 Foundation aims to improve all aspects of burn care in the developing world from prevention to rehabilitation utilising a sustainable approach.

O32.01 Operationalization of joint flexibility problems: an issue illustrated with range of motion data on children after burns

M.K. Nieuwenhuis ${ }^{1}$, A.M. Oosterwijk2 ${ }^{2}$, L.M. Disseldorp ${ }^{1}$, C.J. Van der Schans'2, L.J. Mouton ${ }^{3}$

${ }^{1}$ Association of Dutch Burn Centres, Burn Centre Groningen, GRONINGEN, Nederland

${ }^{2}$ Healthy Ageing, Allied Health Care, Nursing, Hanze University Applied Sciences, GRONINGEN, Netherlands

3University of Groningen, University Medical Center Groningen, Center for Human M, GRONINGEN, Nederland

Objectives: Scar contractures are a well-known sequela of burn injury. To evaluate the effect of (new) treatments or analyse prevalence and risk factors of scar contractures, the existence and severity 
of scar contractures are based on the assessment of joint range of motion (ROM). Most often a ROM of one or more degrees lower than the maximal ROM for that joint and movement direction is considered a problem, but other more function related operationalisations are being proposed. The current study evaluated the effect of applying different operationalisations of joint flexibility problems on its prevalence.

Methods: ROM data of 95 joints affected by burns of 23 children (6-18 years) were used, and data on 18 functional activities (Burn Outcome Questionnaire (BOQ)). To operationalise joint flexibility problems 5 methods were used: 1) ROM below normative ROM, 2) ROM below normative ROM minus 1SD, 3) ROM below normative ROM minus 2SD, 4) ROM below functional ROM, and 5) a score of 2 or more on the Likert Scale of the BOQ. Prevalences were calculated on group and joint level.

Results: Prevalence of joint flexibility problems on a group level based on Method 1 was $100 \%$ or ranged from $13 \%$ to $61 \%$ using Methods $2-5$. Per joint and movement direction, it ranged from $40 \%$ to $100 \%$ (Method 1) and from 0\% to 80\% (Methods 2-4). Parents of four children (18\%) scored that the performance of some tasks were 'a little hard' (i.e., '2' on 4-point Likert Scale) (Method 5).

Conclusion: The prevalences of flexibility problems is highly dependent on the operationalization used, both on group and joint level. To enable comparisons and evaluation of care, a worldwide accepted operationalization is required. As the significance of scar contractures lies in their limiting effect on function, we strongly plead for an operationalization based on function.

O32.02 Multidisciplinary approach in the diagnosis and treatment of nontraumatic spinal-cord injury following high voltage electrical injuries

\footnotetext{
A Monte Soldado, M.L. Torrent Bertran, J Serracanta Domènech, J Aguilera Sáez, L Grossi Garriga, B.P. Dos Santos Marcano, J.P. Barret Nerin

Vall d'Hebron University Hospital, BARCELONA, Spain

Objectives: To describe our multidisciplinary approach in the diagnosis and treatment of patients with nontraumatic spinal-cord injury (SCI) following high voltage electrical injuries.

Methods: We report four cases of patients which suffered from delayed spinal-cord injury after electrical burns. All of them were managed by a multidisciplinary team comprised of Plastic Surgeons from our Burn Unit, Rehabilitation professionals (composed by physicians, physiotherapists and occupational therapist) from our Spinal Cord Injury Unit and Rehabilitation Burn Unit, dietician, psychologist, social worker and nursing staff. This allowed an early diagnosis and implementation of specialized Rehabilitation treatment, focused on: improvement of range of motion, muscular and standing balance, activities of daily living training, splinting and prosthetics, etc.

Results: The four patients suffered from severe electrical injuries that affect both upper and lower limbs; all of them required amputation of one or the two upper limbs. Diagnose of SCI was done between the 10th and 20th day postburn by physical examination and neurophysiological studies. In all the cases the injury was incomplete, according to the ASIA scale. None of the patients showed spinal cord damage or vertebral fractures in the spinal MRI. After rehabilitation program, all patients improved ASIA scale classification grade but still presented important functional sequelae, caused not only by the SCI, but also by the severe burns and amputations suffered.

Conclusion: Delayed Spinal Cord Injury without osteoligamentous lesion is a rare but serious complication following high voltage electrical injuries. Diagnosis requires high clinical suspicion, and is based on a thorough neurologic examination and neurophysiological explorations (that usually shows alterations in somatosensory evoked potentials). In these patients, teamwork and an early Intensive Rehabilitation Program can improve ASIA spinal cord injury classification grade and functionality, with a great impact in the outcomes of this patient specific population.
}

O32.03 Early therapy experience with NovoSorb ${ }^{\mathrm{TM}}$ BTM

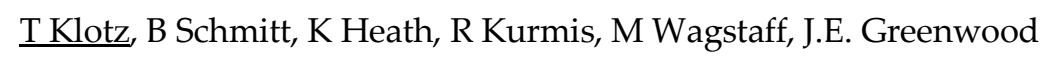

Royal Adelaide Hospital, ADELAIDE, Australia

Objectives: Since 2014, NovoSorb ${ }^{\mathrm{TM}}$ Biodegradable Temporising Matrix (BTM) has been used at our center to treat specific deep burn wounds. Patients were then treated using pre-existing standard 
therapy protocols. As a new dermal substitute, the implications for anticipated rehabilitation times, therapy pathways and scar management compared to our previous treatments were unknown.

Our aim was to map the pattern of recovery following BTM application compared to a historic matched burn cohort.

Methods: Patients who received BTM during their burns admission were identified from existing department records. Matched, retrospective patients were identified based on burn size (total body surface area (TBSA)), depth, age, and gender from our burns database, with no exclusion criteria. Site, time post BTM application to range of motion (ROM), mobilisation, maximal ROM, scar outcomes, incidence of reconstructive surgery, and length of stay were collected.

Results: Twenty-one patients (15 male, 6 female; mean age 39.3 years) admitted with burns averaging $40.25 \%$ TBSA (34.27\% TBSA full thickness) treated with BTM were included. Historical matched patients represented an average of $60.3 \%$ TBSA burn injury (37.3\% TBSA full thickness). Average length of stay was 88.72 days and 84.7 days in each group respectively. To date, nine patients have completed 12-month follow-up with the Patient and Observer Scar Assessment Scale (POSAS) and Matching Assessment of Scars and Photographs (MAPS) assessments. Mean POSAS scores for the observer component were comparable to published data, whilst mean patient scores were less. Pain and itch scores for scar sites were below those expected and improvements in ROM were seen.

Conclusion: Use of BTM following deep burn injury decreases scar management, rehabilitation and reconstructive surgery requirements. Ongoing evaluation of clinical practice and implementation into practice is required to ensure optimal patient outcomes are achieved.

Picture 1: https://www.eventure-online.com/parthen-uploads/89/9EBA/add_1_539762_56f67814f0dd-4507-9e4e-0619cd1328a7. Flexion ROM for BTM Joints Pre- and Post SSG_.png

O32.04 Rehabilitation and multidisciplinairy treatment within the context of the International Classification of Functioning, Disability and Health (ICF); evaluation of a case report

M.A.H. De Wit, J.M. Borst- van Breugel

Red Cross Hospital, BEVERWIJK, Nederland

Objectives: To describe the process of clinical decision making within the context of the ICF framework, in a patient with severe burns.

Methods: We present a case report of the rehabilitation of a 65-year-old women with deep third and fourth degree burns of $28,5 \%$ total body surface area (TBSA) on her trunk, both legs and feet. After seven days a lower limb amputation was performed due to fourth degree burns. She was hospitalized for two months, of which six weeks on the intensive care. She was dismissed to a rehabilitation clinic.

Results: Rehabilitation of a complex patient in the burn centre requires good collaboration between paramedics, mutually, and medical professionals. Rehabilitation is a dynamic process, that involves a lot of clinical decision making, in an effort to provide best practice for the patient. During the rehabilitation in this case report there were multiple factors that were a barrier to the treatment and the patient's overall progress. When reflecting on this complex patient, different issues arise. Multidisciplinary collaboration was a challenge due to limited load capacity and multiple treatment goals. Rehabilitation was hampered due to long intubation of the patient, impaired cognitive functioning and low motivation of the patient. To combat this we used the ICF framework to order body functions, activities, participation, environmental and personal factors

Conclusion: Organizing individual patient problems within the context of the ICF framework and environmental and personal factors provides insight into clinical decision making and facilitates obtaining of a best practice treatment plan.

O32.05 Feasibility and intra- and inter-rater reliability of bedside ultrasonography to assess changes in muscle size in (critically ill) patients with burns

F. G. Bosch ${ }^{1}$, H. Jager-Wittenaar2, C. P. Van der Schans², G. C. Wesseling-keuning3, M. K. Nieuwenhuis ${ }^{1}$

${ }^{1}$ Association of Dutch Burns Centers, Burn Center Groningen, Martini Hospital, GRONINGEN, Netherlands 
2Research Group Healthy Ageing, Allied Health Care and Nursing, Hanze University, GRONINGEN, Nederland

3Burn Center Groningen, GRONINGEN, Netherlands

Objectives: Burn injury causes a persistent and prolonged hypermetabolic state and increased catabolism, resulting in muscle loss and malnutrition. Muscle loss and malnutrition are associated with impaired physical functioning and decreased quality of life. Anabolic competence is required to improve muscle and nutritional status, which is positively influenced by optimal nutrition, sufficient physical activity, and good hormonal milieu. To enable adequate assessment and monitoring of muscle and nutritional status, reliable and valid bedside tools are needed. However, currently no nutritional parameter is available to evaluate skeletal muscle status in patients with burns. Bedside ultrasonography (US) is gaining interest as technique to evaluate loss and recovery of skeletal muscle size (thickness/cross-sectional area). However, evidence on the feasibility and reliability of bedside US muscle assessment in patients with burns is lacking. Patients with burns, certainly the critically ill, pose additional challenges compared to populations in which (bedside) ultrasonography has been used so far. Therefore, we aim to assess feasibility and intra- and inter- rater reliability of bedside US derived muscle thickness assessment in (critically ill) burn patients.

Methods: For this observational, longitudinal study, adult burn patients with $5 \%$ or more of their total body surface area (TBSA) burned are eligible. Skeletal muscle thickness is measured at maximally 4 different body sites: upper arm, lower arm, upper and lower leg, on day of admission, post burn day (PBD) 1, 3, 7, 10, and subsequently weekly until discharge. Only non-burned sites are assessed. To determine intra- and inter-reliability, assessments are performed either twice by the same observer or by two independent observers. Intra- and inter-reliability is evaluated using intraclass correlation coefficients (ICCs) and Bland-Altman plots. Feasibility is assessed by the number of successful measurements and total assessment time per site, during various phases of hospitalization.

Results: Preliminary results will be presented.

Conclusion: Preliminary results will be presented.

O32.06 Improve burn care and patient outcome by combining knowledge from physical therapists and dieticians

G.C. Wesseling-Keuning, K. Bouwmeester

Burn Center Groningen, Martini Hospital, GRONINGEN, Netherlands

Objectives: Severe burns cause a pathophysiological stress response with inflammatory and endocrine aspects. These aspects coupled with numerous surgical interventions and prolonged immobilization cause profound metabolic dysfunction (hypermetabolism and muscle catabolism). This results in significant erosion of lean body mass, muscle weakness, immune depression, poor wound healing and general deconditioning. (Perreira 2005, Porter 2014). Loss of musclemass can be the result of a decrease in muscle protein synthesis (MPS), an increase in muscle protein degradation or a combination of both.

Methods: In burns, loss of muscle mass is driven by an accelerated rate of protein breakdown. The increased release of Ammonic Acids (AA) from protein breakdown provides a stimulus for accelerated MPS. Protein synthesis cannot match the increased rate of muscle protein breakdown. The catabolic loss of muscle can be ameliorated firstly if the uptake of AA from the blood is increased by intravenous infusion, or the digestion of enterally administered proteins, peptides or AA's. These sources of AA may then stimulate protein synthesis to offset the accelerated rate of protein breakdown and AA oxidation. (Weijs 2014)

Secondly, exercise, specifically progressive resistance exercise training (RET), also has an important role in MPS. (Chale, 2013, Porter 2014).

Results: Moreover, RET combined with protein and AA's supplementation ingestion has shown to be highly effective for increasing muscle mass, muscle strength and functional capacity (Little, Philips 2009). To maximize the increase in MPS following RET, appropriate nutrition should be provided in close temporal proximity to each training (Cribb-Hayes 2006, Dreyer, 2008). 
Conclusion: As RET and AA's - and protein supplementation leads to improvement of the functional capacity, there should be an optimal co-operation between physical therapists and dietitians. Multidisciplinary cooperation, also in this area of burn care, is highly recommended.

O32.07 Early exercise rehabilitation during the acute phase of severe burns: a European survey of current practice

DR Schieffelers, E Van Breda, N Gebruers, J Meirte, U Van Daele

University of Antwerp, ANTWERP, Belgium

Objectives: Despite the clinical trend towards early exercise in the acute care of burns, it remains unclear how and to what degree this is currently carried out. In the absence of concrete exercise guidelines, prescribed exercise parameters such as exercise type, intensity, timing remain ill-defined. This study aimed to identify the current practice of early exercise in European burn centres.

Methods: An electronic survey was distributed by the European Burn Association to burn centres throughout Europe, including questions related to resistive and aerobic exercise prescription during the acute phase of severe burns.

Results: 59 burn clinicians, representing 8 of the 25 emailed countries (28,5\%), responded. All respondents reported using some form of active exercise in acute burn patients, but only $31 \%$ of respondents reported commencing active exercise in intubated patients. The provision of active exercise increased to $87 \%$ once out of bed mobility was allowed, and to $100 \%$ once patients were allowed to leave their hospital room. In-/exclusion criteria for active exercise differed greatly among clinicians and were only used by $41 \%$ of respondents. The methods used to determine exercise intensity varied considerably with patient tolerance $(88 \%)$, heart rate $(16 \%)$, general exercise guidelines $(16 \%)$, Vo2max (4\%) being reported for aerobic exercise, and patient tolerance $(59 \%)$, manual muscle testing $(28 \%)$, repetition maximum $(12,5 \%)$, dynamometry $(9 \%)$ reported for resistive exercise. Assessment of muscle force, muscle wasting, and insulin sensitivity were reported by $59 \%$, $13 \%$, and $8 \%$ burn clinicians, respectively, and the employed frequency and methods were extremely variable.

Conclusion: While early exercise has become an integral part of successful burn care, large variation exists in terms of exercise type, intensity, timing, starting criteria, and outcome measures. This study exposes the need for concrete exercise guidelines, and lays the groundwork for future trials investigating parameters of optimal exercise prescription that may improve burn outcomes.

O32.08 Passive in-bed cycle ergometry exercise of mechanically ventilated burn patients at the Burn Center, Uppsala University Hospital, Sweden. A case series.

E.M. Sundin

Uppsala University Hospital, UPPSALA, Sweden

Objectives: The aim of the study was to assess the effect of passive in-bed cycle ergometry exercise on mechanically ventilated burn patients regarding heart rate (HR), mean arterial pressure (MAP), respiratory rate (RR), and blood gas values as pseudo-variables of safety.

Methods: A Single-Subject Experimental Design study was conducted. Five consecutively admitted critically ill adult burn patients on mechanical ventilation were included (33-88 years of age, 3 men, TBSA\% burned: 12-58\%). Data were collected during the patients' first session of passive inbed cycle exercise with the MOTOmed Letto 2 (RECK, Betzenweiler, Germany). The intervention was conducted within 1-26 days (median 3 ) after burn injury.

Results: A comparison between baseline measurements and values during intervention show an increase in mean HR for all patients (2.7 (0.3 to 5.5) bpm). An increase between 4 and $12 \mathrm{mmHg}$ in MAP was seen in three patients and a decrease around $5.5 \mathrm{mmHg}$ in two patients. Three patients increased their RR (1.7 to 5.5) breaths per minute, one decreased $(0,8)$, and one had controlled mechanical ventilation and thus no change. Arterial blood gases showed only minor changes from baseline values, $\mathrm{PaO}_{2}:+0.6$ (range: -1.8 to +2.3 ) $\mathrm{kPa} ; \mathrm{PaCO}_{2}:-0,09$ (range: -0.3 to +0.4 ) $\mathrm{kPa} ; \mathrm{SaO}_{2}:+0.7$ (range: -1.7 to +3.2$) \%$. Lactate and $\mathrm{pH}$ did not change compared to baseline values. 
Conclusion: All patients managed to maintain the passive in-bed cycle exercise for 20 minutes, as recommended for ICU patients, without deviating from the individually preset thresholds for aborting the intervention. Early in-bed cycling exercise for mechanically ventilated burn patients at the BICU is considered to be a clinically safe procedure since effects on the physical variables were negligible. However, further investigations are needed.

O33.01 Evaluation of the treatment of deep thermal Hand burns in Adults with NexoBrid in the Dutch population (HAND-study) using a standardized hand assessment tool

J. M. Borst- van Breugel

Red Cross Hospital, HEERHUGOWAARD, Netherlands

Objectives: Our objective is to present our standardized hand assessment tool for the evaluation of hand burns after enzymatic debridement (ED), used in the HAND-study.

Methods: Patients with deep hand burns treated with ED were subjected to different hand assessment tests, quality of life instruments, and a scar quality questionnaire to evaluate the outcomes of treatment with enzymatic debridement in deep hand burns. The following tools were used: modified Kapandji Index, goniometry, figure-of-eight-method, Semmes Weinstein monofilaments test, dynamometer, Jebsen-Taylor test, Canadian Occupational Performance Measure, Patient and Observer Scar Assessment Scale, EuroQoL 5D, Burn Specific Health Scale, and the Disabilities of the Arm, Shoulder and Hand scale. Follow-up measurements were at three, six and twelve months after wound healing.

Results: Ten patients were included, nine male, with a mean age of 56.3 (SD 12.5) years. Seven patients had both hands burned, of which four patients received ED of both hands. In 8 patients skin grafting of part of the enzymatic treated hand was required due to deep dermal and/or full thickness burn depth. The median time to wound healing of the enzymatic treated hands was 31.0 (range 24.039.0) days. Follow-up is expected to be completed by September 2019 at three, six and twelve months, in ten, ten and seven patients respectively. By systematically measuring hand function it is possible to evaluate the process.

Conclusion: A hand assessment tool is required to systematically evaluate hand function. Comprehensive and long-term monitoring of the hand function of patients with deep hand burns can lead to an increase in therapy compliance. Moreover, problems are identified earlier on and recommendations can be made for appropriate treatment. We recommend systematic hand function evaluation with a hand assessment tool in every patient with (deep) hand burns.

O33.02 NexoBrid learning curve in a middle income country

A.M. Boiangiu ${ }^{1}$, R. Mihai ${ }^{1}$, A.R. Oporanu ${ }^{1}$, C.G. Bejinariu ${ }^{1}$, A.M. Badeana ${ }^{1}$, C. Giuglea ${ }^{2}$, S.A. Marinescu ${ }^{1}$

${ }^{1}$ Bagdasar-Arseni Clinical Emergency Hospital, BUCHAREST, Romania

2Saint John's Clinical Emergency Hospital, BUCHAREST, Romania

Objectives: This paper intends to present the challenges that medical personnel may encounter when starting to use enzymatic debridement, especially in middle income countries, such as Romania. In these countries, surgeons have to make do with limited resources and this influences the learning curve and the overall result.

Methods: The beginning of our experience was with the Colectiv Club mass casualty fire in Bucharest, in October 2015. We received 23 patients, out of which 17 were treated with NexoBrid. After that, we started using it more frequently, totaling 115 patients until present. We have used the recommendations of the label, however we also have to adapt to our limited resources, lack of medical personnel, as well as the lack of a burn center. We have used NexoBrid especially for upper limbs (106 cases), faces (4 cases), circumferential burns (28 cases), lower limbs and feet (40 cases). We apply it as soon as possible after admission, but we also treat older burns ( $>7$ days). If the burn is fresh, we skip the pre-Nexobrid soaking. On older burns we soak for $4-5$ hours. We remove Nexobrid after 3-6.5 hours, depending on the depth of the burn. We soak the burns with saline, for 2-12 hours 
after NexoBrid (90\% overnight). Allografts and skin substitutes are not available, so we either autograft at 24-72h, or use SSD and wait.

Results: Even though some surgeons were reluctant to use NexoBrid, enzymatic debridement has entered our standard of care. The debridement percentage is $>96 \%$, however the lack of allografts, skin substitutes in the first part of our learning curve, lead to burn conversion (55\%) and autograft loss (19 cases).

Conclusion: The learning curve for using enzymatic debridement can be intimidating, but the discomfort is greatly outweighed by its benefits, especially in circumferential burns or burns in aesthetic or functional areas.

O33.03 Off-label NexoBrid ${ }^{\mathrm{TM}}$ use for enzymatic burn debridement in children

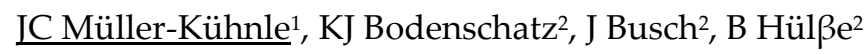

${ }^{1}$ Paracelsus Medical University Nuremberg, NUREMBERG, Germany

${ }^{2}$ Klinik für Kinderchirurgie und Kinderurologie Klinikum Nürnberg, NUREMBERG, Germany

Objectives: Up to date only limited options are available in the initial wound management of severe burns in children. There is evidence for enzymatic wound debridement with the bromelain containing substance NexoBrid ${ }^{\mathrm{TM}}$ in adults, but this treatment is still classified as off-label use in children. For this reason, we started a clinical trial in order to examine and evaluate the use of NexoBrid ${ }^{\mathrm{TM}}$ in children.

Methods: We analyzed the outcomes of $23 \mathrm{NexoBrid}^{\mathrm{TM}}$ procedures performed on the extremities of ten children with severe burns of degree $2 b$ and higher over a period of twelve months.

Results: Our good clinical impression was confirmed by excellent results in the Vancouver Scar Scale in all 23 wounds in terms of pigmentation, vascularity, pliability and scar height at a six-month follow-up examination.

Conclusion: In our experience, neither burn location nor depth were a limiting factor for NexoBrid ${ }^{\mathrm{TM}}$ use. On the contrary, NexoBrid ${ }^{\mathrm{TM}}$ showed beneficial effects in complicated circumferential burn wounds on the distal areas of the forearms which normally indicates a difficult treatment. Based on our results we highly recommend additional use in children in further studies to gather more knowledge about this treatment.

\section{O33.04 ENZYMATIC DEBRIDEMENT (NEXOBRIDR) FOR BURN TREATMENT: TOWARDS A NEW PARADIGM OUR EXPERIENCE IN CRUCES UNIVERSITY HOSPITAL'S BURN UNIT (Vizcaya, Spain).}

\section{Aparicio Elizalde, F. Collía Martín}

Cruces University Hospital, BARACALDO, Spain

Objectives: In recent years, surgical treatment of burns has given way to alternative and less aggressive interventions, marked by the advent of enzymatic debridement (Nexobrid $\left.{ }^{\circledR}\right)$. This paper discusses the experience with enzymatic debridement in Cruces University Hospital's Burn Unit: wound management regime, need of surgery, blood transfusions and sequelae.

Methods: A group of 18 patients with partial, full thickness and mixed burns was treated with Nexobrid ${ }^{\circledR}$ (Group A) between January and December 2018. Group A was compared with group B, composed of another 18 patients who had been surgically treated and who sustained similar burns regarding burned surface, depth, anatomic location and mechanism.

Results: Group A was composed of burns between 1 and 55\% TBSA. The most frequent location was the upper extremity $(\mathrm{n}=13)$. No blood transfusions were needed. An ulterior surgical procedure was required in five cases; in those cases, surgery consisted mostly on autologous skin grafting. To date, no sequelae surgery has been performed. The difference between group A and group B reveals statistically significant results ( $\mathrm{p}<0.05)$.

Conclusion: The advent of the NexoBrid ${ }^{\circledR}$ era in our Hospital shows promising results, decreasing the necessity of surgical interventions and the number of blood transfusions, therefore setting a whole new standard of care. 
O33.05 The treatment of deep dermal burn wounds with Platelet Rich Fibrin (PRF) after enzymatic debridement

AS Schulz, W Heitzmann, J Schiefer, PC Fuchs

Cologne Merheim Medical Centre, COLOGNE, Germany

Objectives: Enzymatic debridement was found to remove burn eschar selectively. Thus, vital tissue can be preserved and burn wounds can heal spontaneously in many cases. However, healing time can be significantly prolonged depending on burn depth and extend. It is therefore questionable, whether the application of Platelet Rich Fibrin (PRF) can shorten the wound healing time and thus improve long term scar quality.

Methods: In a single-centre clinical trial we treated patients with partial thickness to deep dermal burns by enzymatic debridement followed by PRF application. All patients wear compression clothing over 6 months. After 8 and 12 months, the aesthetics and function of the scar were assessed objectively and subjectively.

Results: 30 patients (age min16 years, max 70 years; TBSA min 1\%, max 35\%) with partial thickness to deep dermal burns (among them 10 facial burns) were included in the study. Between $0.5 \%$ and $9 \%$ burn surface was treated enzymatically followed by PRF application. PRF preparation and application was easy to learn even for unexperienced users with some device related issues in the early stage of their learning curve. Patients reported minor pain during procedure without additional anaesthesia. No adverse occurred during any treatment. It was found that by adding PRF, wound healing time could be reduced. We found excellent results in scaring after 8 and 12 months.

Conclusion: The treatment of partial thickness to deep dermal burns with PRF following enzymatic debridement might be suitable to improve wound healing, scar quality and the functionality of the hand after burn injury. The treatment is easy to handle and patients tolerate the treatment well.

\section{O33.06 OUR EXPERIENCE IN ENZYMATIC DEBRIDEMENT OF DEEP DERMAL AND FULL THICKNESS BURNS}

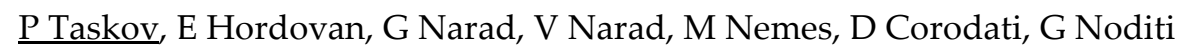

SCJUT CASA AUSTRIA, TIMISOARA, Romania

Objectives: Easy, selective and efficient debridement of deep dermal burns that in the same time preserves viable tissue and anatomical structures.

Reducing hospitalization, costs and need for additional excisional surgery and grafting after EDNX.

Methods: EDNX was developed for the removal of thermic burn eschars and in particular for preserving the viable tissue. The active ingredient is a partially purified proteolytic mixture of bromelain extracted from the stem of pineapple tree, which is allowed in present to treat up to $15 \%$ TBSA.

The active gel was applied over the burn wound using one dose of $5 \mathrm{~g}$ of sterile powder and $50 \mathrm{~g}$ of sterile gel for the enzymatic debridement of a 2,5-5\% body surface.

Results: A total number of 30 patients aged between 18 and 76 with mean of $\sim 30 \%$ TBSA were treated during June 2017 - February 2019.

All patients were admitted on the day of the injury or up to 72 hours after.

Burn depths were framed in IIB degree in $50 \%$ of the cases and IIIrd degree in the other $50 \%$ of the cases.

21 patients successfully finished the treatment, 5 patient needed excisional surgical debridement and grafting and 4 patients have died.

The follow-up was performed at 3 to 6 months after applying EDNX.

Conclusion: Following our experience based on 30 cases we find out that using EDNX on deep dermal burns and full thickness burns is easy, fast, efficient in terms of costs and is performed safely.

Promising results were evaluated regarding the healing potential and early recovery as well as the efficiency and selectivity of debridement.

Reduced healing time, extension, elasticity, retraction and pliability of post burn scars. 
O33.07 The use of Nexobrid in a regional burns centre: our learning with enzymatic debridement of resuscitation and ICU burns.

T K Randawa, C Harris, J Stallard, PM Muthayya, A Phipps, U Anwar

Pinderfields Regional Burns centre, WAKEFIELD, United Kingdom

Objectives: We aim to present our learning with the use of Nexobridâdebridement of burns, and how this has evolved in our centre to include patients with resuscitation and ICU level injuries.We have an established protocol for use of Nexobridâ in resuscitation level injury.

Methods: Identifying patients undergoing nexobrid from our database.Patient Demographics,mechanism and\% TBSA of the burn injury were recorded(resuscitation burn was defined as TBSA $15 \%$ or greater $18-65$ years, $10 \%$ or greater over 65 years),patients requiring burns ICU admission for inhalation injury were also noted.Outcome data,including number of trips to theatre,time to healing and\%TBSA requiring autograft were noted.

Results: We identified a total of 45 patients who underwent Nexobrid debridement of part or all of their burn injury on day 0-2 of admission.\%TBSA debrided with Nexobrid ranged from 2-54\%, of these 16 patients were classed as resuscitation level injuries with\%TBSA ranging from $13-54 \%$,or required ICU admission for inhalation injury.In this group of resuscitation and ICU level injuries; we have observed a similar sequelae; hypertension, increased analgesia requirements and hypotension,requiring inotropic support following application, which is now anticipated in this group of patients. In two patients Nexobridâ was used in lieu of escharotomies to circumferential upper limb injuries. Number of trips to theatre and\%TBSA requiring autograft has been reduced compared with traditional surgical debridement and grafting techniques in a similar injury population.

Conclusion: Nexobrid rapid enzymatic debridement is safe and effective in the resuscitation and ICU level burn injury. We noted during the resuscitation period, a sequelae;hypertension, increased analgesia requirements and hypotension,requiring inotropic support following application.This physiological paradigm is now managed proactively. Use of nexobrid has evolved leading to excellent results, with reduced trips to theatre, reduction in\%TBSA autografted and reduced time to healing.

\section{Abstracts E-Poster Presentations}

P001 Early diagnosis of burn sepsis

$\underline{\text { O.M. Kovalenko }}{ }^{1}$, H.P Kozinets ${ }^{2}$, A.O. Kovalenko ${ }^{3}$, A.M. Boyarska', O.I. Osadcha ${ }^{3}$

${ }^{1}$ National Medical University by O.O.Bogomolets, KYIV, Ukraine

${ }^{2}$ National Medical Academy of Postgraduate Education, KYIV, Ukraine

${ }^{3}$ Burn Clinic, KYIV, Ukraine

Objectives: Sepsis and septic shock are the main causes of mortality in patients with severe burns. The search for markers for the early diagnosis of burn sepsis was relevant.

Methods: 48 patients with dermal burns 20-70\% TBSA were under supervision. The study group -30 patients. $70 \%$ necrotic tissue was excised within 10 days. Control group -18 patients, necrotic tissue was excised late.

Results: Not less than $70 \%$ deep and superficial necroses were excised in patients of study group within 10 days after injury. Level of prokalcitonin (PCT) $\leq 2 \mathrm{ng} / \mathrm{mL}$, C-reactive protein (CRP) $\leq 100$ $\mathrm{mg} / \mathrm{L}$, number of microorganisms in burn wounds $\leq 10^{4} / \mathrm{g}$, plasma lactate to $2.7 \mathrm{mmol} / \mathrm{l}$ were determined. Toxic granulation neutrophils(TGN) $\geq 45 \%$ and nitro-blue tetrazolium (NBT) test $\geq 25 \%$ indicate presence of a systemic inflammatory response with possible septic complications. The clear correlation noted between blood lactate levels and development of RDS and MOF. Organ dysfunction decreased to 3.6 points SOFA. High number of microorganisms burn wounds $\geq 106 / \mathrm{g}$ was determened in the control group of patients and it was correlated with severity to 8.95 points SOFA, CRP - above $200 \mathrm{mg} / \mathrm{L}, \mathrm{PCT} \geq 10 \mathrm{ng} / \mathrm{mL}$. CRP higher than normal, was $70.1 \pm 10.2 \%$ of patients. Increased plasma lactate from 2.7 to $4.0 \mathrm{mmol} / \mathrm{L}$ indicating risk zone, more than $4 \mathrm{mmol} / \mathrm{L}$ - of sepsis, $\mathrm{TGN} \geq 60 \%$ and NBT- test $\geq 25 \%$ are seen in cases of sepsis with multiple organ failure. 
The degree of MOD remained at high level to 8.6 points. High correlation identified with the level MOF and PCT $\left(\mathrm{r}_{1}=0,7\right)$ and CRP $\left(\mathrm{r}_{2}=0,9\right)$.

Conclusion: Result of treatment depends on early surgical tactics. Bacterial colonization wounds in $105 / \mathrm{g}$, PCT level $\geq 2 \mathrm{ng} / \mathrm{mL}, \mathrm{CRP} \geq 100 \mathrm{mg} / \mathrm{L}$, lactate $\geq 4 \mathrm{mmol} / \mathrm{L}$ TGN $\geq 60 \%$ and NBT- test $\geq 25 \%$ are seen in cases of sepsis with multiple organ failure.

P002 Role of steroid for the treatment of severely burned

$\underline{\text { O.M. Kovalenko }}{ }^{1}$, A.M. Boyarska², H.P. Kozinets ${ }^{3}$, A.O. Kovalenko², O.I. Osadcha ${ }^{2}$

${ }^{1}$ National Medical University by O.O.Bogomolets, KYIV, Ukraine

2Burn Clinic, KYIV, Ukraine

${ }^{3}$ National Medical Academy of Postgraduate Education, KYIV, Ukraine

Objectives: In severe burn injury with shock a disturbance in the interaction of the immune and neuroendocrine systems, including increased secretion of adrenal hormones were studied. Secondary immunodeficiency states with inhibition of the cellular link and cytokine disbalance were induced.

Methods: 35 patients with dermal burns 20-70\% TBSA were under supervision. The study group - 20 patients, $70 \%$ necrotic tissue was excised within 10 days. Control group - 15 patients, necrotic tissue was excised late.

Results: An increase in proinflammatory interleukins IL-1 up to $130 \mathrm{pg} / \mathrm{ml}$ and TNF up to 1065 $\mathrm{pg} / \mathrm{ml}$ from the first day of the injury were determined. The level of IL-10 to $70 \mathrm{pg} / \mathrm{ml}$ by $8-10$ days after injury indicates the development of immune response suppressing reactions.

An increase in the level of cortisol to $850 \mathrm{nmol} / 1$ in the 1st day was observed, which survived after the removal from shock. For two weeks after the injury gradually decreased. A high concentration of cortisol is the natural response of the neuroendocrine system.

The level of cortisol below $280.0 \mathrm{nmol} / 1$ in the 1st day is a prognostically unfavorable sign, indicating a high probability of death and septic complications right up to septic shock. Septic shock may be complicated by 1) an occult adrenal insufficiency, 2) a glucocorticoid peripheral resistance syndrome.

Correlation of cortisol and cytokine IL-10, as the "key" factor of the immunosuppressive phase of systemic inflammation, indicating risk of complications.

Conclusion: Our study demonstrated the efficacy and tolerability of hydrocortisone replacement therapy (50 mg intravenously bolus four times a day) is given for 10 days and its positive effect on systemic inflammatory response.

P003 Visual loss in major burn injury after enzymatic and surgical debridement

A Fernández-Quinto, C García-Sancho, P Cerdeira-Pena, ME López-Suso, JJ García-Barreiro, R Galeiras-Vázquez

Complejo Hospitalario Universitario de A Coruña, A CORUÑA, Spain

Objectives: Review of an extremely rare complication in a major burn injured patient due to a case: ischemic optic neuropathy (ION).

Methods: A 37 -year-old male with a $85 \%$ total body surface burn injury caused by high temperature water vapor with caustic soda was admitted to the Burn Care Unit of our center. He underwent initial volume fluid resuscitation with 27L of Lactate Ringer and needed invasive hemodynamic monitoring due to refractory shock.

Escharotomy was needed the day of admission, and sequential enzymatic debridement with bromelain twice and debridement with coverage five times were conducted during hospitalization.

After the organ failure and skin coverage being solved, he was extubated on 85 postburn day (PBD).

He received ophthalmologic follow-up, with normal intraocular pressure, fundoscopy and anterior pole without alterations.

Results: On 92 PBD the patient complained of blindness. Intraocular pressure was $17 \mathrm{mmHg}$, fundoscopy showed disc pallor; carotid arteries doppler ultrasound and CT scan had no alterations and magnetic resonance showed nonspecific minimal widen of subarachnoid perioptic spaces. 
Dismissed pharmacologic, compressive and infectious causes, the patient was diagnosed of ischemic optic neuropathy.

There is not an effective treatment available and the complication is currently irreversible.

Conclusion: Non-arteritic ION is a rare cause of visual loss due to a decreased blood flow through the optic nerve. It has been described in other groups of postoperatory patients and as a major complication in polytraumatisms that need massive fluid resuscitation, but barely described in burned patients. Currently there is not an effective treatment available for this complication therefore prevention is the principal objective.

P004 Sedoanalgesia for enzymatic debridement procedures. Our experience.

ISA SAEZ AVILES, ASB Sánchez Balado, IFF FUENTES FERNANDEZ, JGJ GARCÍA JUARRAN, MUO UMAÑA ORDOÑEZ, CRA RECALDE ANDRES, ELB LORDA BARRAGUER

ALICANTE GENERAL HOSPITAL, ALICANTE, Spain

Objectives: Nexobrid (MediWound Ltd, Israel) enzymatic debridement is nowadays increasing for the treatment of deep burns. General anesthesia is considered due to pain management, limiting the early use of the product, which may compromise the outcome.

We present our experience using a combination of midazolam and ketamine in our Burn Care Unit for Nexobrid application.

Methods: A total of 16 patients have been treated with Nexobrid under sedation with a combination of ketamine $(1 \mathrm{mg} / \mathrm{kg})$ and midazolam $(0,05 \mathrm{mg} / \mathrm{kg})$. Once recovered after the procedure, an anonymous questionary about subjective pain was given, with 0 being no pain and 10 maximum pain. Patients ASA $\geq 3$, with neurological or respiratory impairment, were excluded.

Results: Average pain before the procedure was $6,25 \pm 1,84$. It tends to decrease with the

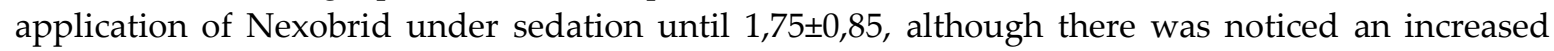
when the product is washed out after 4 hours, reaching a value of 2,375 $\pm 1,14$. We registered 2 patients with minor adverse effects such as nightmares or irritation, whereas 14 referred to have no memories.

Conclusion: Ketamine and low dose midazolam combination during the Nexobrid application procedure is considered to be a safe and more available technique in patients low-risk ASA patients and without neurological or respiratory diseases. Pain has been well tolerated, without any major adverse effects registered. Nor operation theatre neither postoperation recovery was needed.

P005 Enzymatic Debridement (ED) at Bedside: Role of Dexmetomidine during its application in Pediatrics with Burn-Induced Compartimental Syndrome: Case Report.

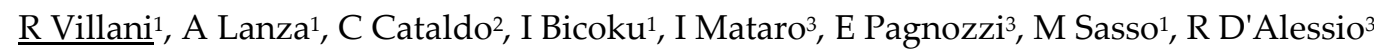

${ }^{1}$ A. Cardarelli Hospital, NAPLES, Italy

${ }^{2}$ University L Vanvitelli, NAPOLI, Italy

${ }^{3}$ Burn Unit A Cardarelli, NAPOLI, Italy

Objectives: Avoiding pain and discomfort should be a primary gold standard in management of all burned patients: in Our Centres application / removal of mixture for Enzymatic Debridement (ED) in Adult ones follows our procedural pain relief schedule by ketamine/fentanyl-low-dose/midazolam compound usually used for dressing change/wound debridement, measuring Richmond AgitationSedation Scale (RASS), Aldrete Score and VAS in order to set Comfort, Clinical status and Analgesia. Although off-label in Pediatrics, after Informed Consent, mixture for ED was apply in a 20\% TBSA 13 y.o. boy with left forearm burn induced compartment syndrome, measured by ICP monitoring; added to drugs using dexemetomidine, $\alpha 2$ - adrenergic agonist allowed comfort in lapsetime of 4 hours between application/removal procedures.

Methods: 13 yo boy, $38 \mathrm{Kg}$, admitted in Burn Intensive Care Unit after primary survey, with $20 \%$ TBSA Alcool induced burns, history of low mental delay, no allergies. At bedside, in spontaneous breathing, monitoring Vital Parameters (HR, SpO2\%, RR, NIBP) and in left circumferential burned forearm measuring of intracompartimental pressure ( ICP) that shows high level of $45 \mathrm{~mm} \mathrm{Hg}$. Infusion of dexmetomidine $0.5-0.9 \gamma / \mathrm{k} / \mathrm{h}$ was started to avoid discomfort and prior to application of ED mixture in ketamine/ fentanyl /midazolam (K: 1,5-2 mg/k; F: 0,5-1 mcg/kg; M 0.15mg/kg). 
Results: Application of debridement mixture was applied on 15\% TBSA included on circumferential burned left forearm that shows even after $2,5 \mathrm{~h} \mathrm{ICP}$ of $10 \mathrm{mmHg}$. In lapsetime comfort of patient was obtained by increasing dexmetomidine at high level of infusion admitted in patient in spontaneous breathing. Removal of Enzymatic Debridement was implemented at same way of application.

Conclusion: Aldrete Score, RASS score and VAS score showed a good comfort and analgesia, showing a gold standard tailored patient therapy for a less invasive surgical technique at bedside.

P006 Usage of exogenic creatinephosphat in perioperative care in burn ICU patients.

SB Balogova

Hospital of Kosice-Saca, KOSICE, Slovak Republic

Objectives: Our poster show usage of creatinephosphat in pre- peri- and post-operatice care in burn patients

Methods: Creatinephosphat in exogenic form in middle dosage $2-4 \mathrm{~g}$ per day was used in patients before and after surgery in burn ICU to maintain levels of energy and maintain condition of kardiomyocytes and skeletal muscles.

Results: Separately, we evaluated cases of patients in burn ICU with multimodal therapy.

Conclusion: Exogenic creatinephosphat is fast and safe way of supplementing of energy and is supporting therapy in multimodal process in burn care.

P007 The Diagnostic Scale of Sepsis in Patients with Burn Disease

$\underline{\text { Y. V. Zhylinski }}{ }^{1}$, A. V. Hlutkin², U. U. Hurinovich³, A. V. Gubicheva4, P. V. Skakun ${ }^{4}$

${ }^{1}$ Minsk City Clinical Emergency Hospital, MINSK, Belarus

${ }^{2}$ Grodno State Medical University, GRODNO, Belarus

${ }^{3}$ Stolbtsy Central District Hospital, STOLBTSY, Belarus

${ }^{4}$ Belarusian State Medical University, MINSK, Belarus

Objectives: Development of a scale for diagnosis of sepsis in patients with burn disease and evaluation of its effectiveness.

Methods: Retro-prospective cohort study was conducted. 76 burned patients were included in the study to develop a sepsis diagnostic scale. The diagnosis of sepsis was exhibited according of Chinese Medical Association criteria. The medical and economic analysis of the proposed scale was based on data from 80 patients with sepsis. The retrospective group included 39 patients, the prospective group - 41. The groups were uniform in age and sex composition, the patients received an equivalent burn injury, which caused the development of burn disease.

Results: We proposed a scale for diagnosis of sepsis in patients with burn disease, which includes the definition of clinic-laboratory criteria for inflammatory response and organ dysfunction (hypo- or hyperthermia, tachycardia, hyperglycemia, hypernatremia, thrombocytopenia, left leukocyte shift, respiratory failure, enteral failure, impaired mental status) and a presepsin test. If there are 5 or more criteria for a systemic inflammatory response and organ dysfunction and the diagnostic level of presepsin is exceeded, sepsis is diagnosed. The proposed scale has a high sensitivity $-94.1 \%$ and specificity $-93.3 \%$, AUC $=0.95$, provides early diagnosis of sepsis in patients with burn disease, does not require the use of long-term research methods, and also allows monitoring the effectiveness of the treatment. Using the developed scale in clinical practice, the cost / effectiveness ratio was reduced in 1.4 times compared with the standard diagnostic method.

Conclusion: The developed scale is a diagnostic model of excellent quality. Using the developed scale led to an increase in the effectiveness of treatment of severely burned patients in 1.4 times.

\section{P008 HEMODYNAMIC MONITORING OF SEVERE BURN PATIENTS: SHOULD WE USE ARTERIAL LINE?}

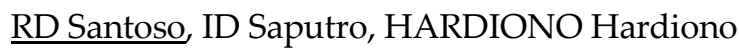

Premier Surabaya Hospital, SURABAYA, Indonesia 
Objectives: Accuracy of non invasive blood pressure (NIBP) in severe burn patients can be difficult so that newer dynamic measurements of invasive blood pressure (IBP) hold promise for determining fluid status and fluid responsivenes. This study aims to assess whether arterial line shoud be placed in severe burn patients.

Methods: Case report: A 61-year-old female was referred from a rural hospital four days after injury with Burn grade II AB TBSA 54,5\% and inhalation trauma due to household stove explosion in closed space. She started to develop respiratory distress and sepsis. Her urine output was decreasing, whereas systolic NIBP stayed above $100 \mathrm{mmHg}$ on fluid resuscitation and multiple vasopressors. She fell to septic shock and an arterial line was placed.

Results: NIBP is insufficient to monitor hemodynamically unstable, septic, on multiple vasopressors, and severe burn patients. Therefore, arterial cannulation may be beneficial for patients who are responding poorly to therapy or requiring higher-dose vasopressor when tight control of hemodynamic response is essential. More advanced dynamic hemodynamic monitorings are not usually available. The common site of arterial line is radial and femoral. Complications of arterial line placement occur approximately $1-3 \%$

Conclusion: While hourly urine output is still used as the endpoint of resuscitation, invasive BP monitoring is needed whenever accessible. Arterial line is an acceptable intervention and relatively safe procedure to show pulse pressure variation to predict fluid responsiveness in fluid resuscitation.

P009 Assessing a new pain management protocol.

J Carbajal-Guerrero, P Gacto-Sanchez, S Escalona-Rodríguez, M Mendoza-Prieto

Hospital Virgen del Rocio, SEVILLA, Spain

Objectives: Clinical guidelines in burns analgesia strongly recommend analgesia protocols creation in every burn unit. Those should be specific for different pain modalities present in burned patients. In October 2016, our Burn Unit analgesia protocol was updated. PCA systems (patient controlled analgesia) were introduced as main changes, both for analgesia at admission and postoperatively. Besides, analgesia with opioids of sustained and rapid release was established for the interoperative periods and specific guidelines for sedation during the dressing changes were created. A numerical rating pain scale (NRS) was used twice a day for every patient. Pain objective was established at being $\leq 4$.

The objective was to assess the effectiveness of the new analgesia protocol through NRS data analysis.

Methods: Between February 2017 and June 2018, 46 patients pain assessment data were reviewed up to a maximum of their first month stay. Burned body surface area (BSA), burn etiology, the need for urgent escharotomy, number of surgical procedures and data regarding NRS values were recorded.

Results: Mean NRS values during their hospital stay at the Burn Unit was 3.9. 60\% of the time of stay in the unit, patients were maintained with NRS values $\leq 4$. This percentage showed an inversely proportional relationship with the BSA. Research regarding different analgesics did not reach statistical significance. Registration of NRS scores were incomplete as patients evolution were improving.

Conclusion: Although the main objective of the protocol update was partially achieved, there is still a wide margin for improvement, both in achieving lower NRS scores and in collecting them.

P010 Bilateral recurrent laryngeal nerve and unilateral hypoglossal nerve palsy (Tapia's Syndrome) secondary to intubation in Burns Intensive Therapy Unit (ITU).

DC Hathaway, N Ibrahim, S Hemington-Gorse, JJ Cubitt

The Welsh Centre for Burns and Plastic Surgery, SWANSEA, United Kingdom

Objectives: First described by otolaryngologist Antonio Garcia Tapia, Tapia's syndrome (TS) was reported as an ipsilateral larynx and tongue hemiplegia originating extracranially $(1,2)$. This rare eponymous syndrome has since been applied to other derivatives of similar pathology. The proposed mechanisms of injury are tracheal intubation, abnormal head/neck positioning and high endotracheal tube cuff pressures $(3,4)$.

Methods: We report a case of a 16-year-old male with $42 \%$ total body surface area mixed-depth 
burns to his legs, abdomen, back and hands following an explosion. He was intubated on presentation to the Emergency Department with minimal tracheal soiling not requiring lavage. He underwent a total of 10 theatre sessions during his ITU admission.

Whilst intubated, he received treatment for a ventilator-acquired pneumonia and a malodourous tonsillar exudate culture grew Fusobacterium necrophorum. Extubation was successful on the third attempt at day 19. He was found to be aphonic with minimal laryngeal elevation, no anterior hyoid movement and a weak cough causing a dysfunctional swallow. Laryngoscopy identified sluggish vocal cords bilaterally. A left hypoglossal nerve palsy was noted on day 32 .

Magnetic resonance imaging of his cervical spine and skull base showed no structural cause for the patient's symptoms which resulted in a diagnosis of TS.

Results: There has been some improvement in hyolaryngeal elevation although the patient is heavily reliant on Yankauer suctioning. Tongue denervation persists, his voice remains aphonic and airway protection is severely compromised requiring nasogastric feeding.

Conclusion: This patient underwent multiple intubations and visits to theatre which may have contributed to his presentation with TS. In reported cases of TS, symptoms resolve in 3-6 months $(5,6)$. There has been limited improvement in the patient's symptoms six weeks following extubation.

The growth of Fusobacterium necrophorum, which can rarely cause Lemierre's syndrome (including CN IX, X, XII palsy) was a potential differential diagnosis that was excluded.

P011 Approach to airway management in burns.

$\underline{\text { R Pinto-Lopes }}^{1}$, P Frazão ${ }^{2}$, J Sousa Correia ${ }^{3}$, P Egipto ${ }^{4}$

${ }^{1}$ St Andrews Centre for Burns and Plastic Surgery, CHELMSFORD, United Kingdom

${ }^{2}$ Centro Hospitalar do Baixo Vouga, AVEIRO, Portugal

3Instituto Português de Oncologia do Porto Francisco Gentil, PORTO, Portugal

${ }^{4}$ Centro Hospitalar Universitário de São João, PORTO, Portugal

Objectives: The burn patient Is a polytrauma and their approach should follow established trauma algorithms. The primary survey and stabilisation aim to identify and treat life threatening situations. Airway assessment is therefore a priority and early intubation plays an important role in symptomatic patients with inhalation injuries or in facial, oral and oropharyngeal burns that compromise airway patency. Nonetheless, intubation is not innocuous. Therefore, we should weight risks and benefits to avoid unnecessary procedures and iatrogenesis.

Methods: Referred patient with burn sustained after gas cylinder explosion. ABCDE approach performed according to ATLS guidelines. Early orotracheal intubation and resuscitation according to Parklands formula were performed according to findings. When haemodynamically stable, the patient was transferred to a specialised burns centre for subsequent management.

Results: Total burn surface area of $26 \%$, with superficial and superficial partial thickness burns to head, neck, upper chest, both upper limbs (circumferential), left face and lower back. Dysphonia and glossal oedema were present warranting orotracheal intubation. Laryngoscopy grade III, with oedema of the base of the tongue and supraglottic area with narrowing of the lumen of the upper airway tract. Fluid resuscitation was initiated according to Parklands formula. When in specialised burn centre, bronchofibroscopy performed showed no alterations of the tracheobronchial tree, namely oedema or erythema. Twenty-six hours after burn patient was extubated safely due to absence of glossal oedema, direct laryngoscopy with no evidence of oedema/erythema of upper airway tract.

Conclusion: Airway patency assessment is paramount in the assessment of the burn patient. Airway compromise is an indication for early orotracheal intubation. Not to intubate when clinically indicated is associated with greater risks when compared to unnecessary intubation. It's essential to carefully evaluate need for maintaining intubation, after stabilising the patient, and opt for early extubation in the presence of extubation criteria to reduce risk of iatrogenic damage.

P012 Feasibility of extensive Nexobrid application in treatment of body surface area above 15\%.

PK Buehler, DA Hofmänner, M Waldner, H Klein, J Plock 
University Hospital Zurich, ZURICH, Switzerland

Objectives: Bromelain-based enzymatic debridement with Nexobrid is an alternative to standard of care tangential excision of partial and full thickness burns. However, data is limited for large-area applications and not approved for use of $>15 \%$ body surface area. The aim of this retrospective trial was to investigate off label extensive enzymatic debridement and potential side effects.

Methods: Between 01/2017 and 12/2018, 59 patients with deep partial- to full-thickness burns underwent enzymatic debridement (ED) in our Burn Center and were evaluated for systemic inflammatory reaction, adverse effects, hemodynamic instability and electrolyte shifts. The patients were categorized into two groups, the authorized group (AG) with a treated area below $15 \%$ TBSA and the off label (OG) group with an area above 15\% TBSA debrided in one session.

Results: In total, 49 patients were treated in the AG group with an area of median 6\% (IQR 3 to 10) and 10 patients were treated in the OG group with an area of median 18\% (IQR 15 to 29). There were no significant differences in heart rate, blood pressure, temperature and hemodynamic instability. No treatment-related serious adverse events were observed in both groups. Catecholamine therapy was similar in both groups. No differences in the laboratory parameters of leukocytes, CRP, PCT and lactate before application and in the following three days were observed. Sodium, potassium, chloride and phosphate levels did not differ. There was no evidence for an electrolyte shift in both groups.

Conclusion: The extensive application use of Nexobrid did not result in any expected or unexpected side effects in the investigated patient groups. These preliminary results indicate potential safety and efficacy of bromelain-based enzymatic debridement in treatment of TBSA areas above $15 \%$ in severe burns in one session.

P013 Analgesia Protocols for Burns Dressings: The Lack of Efficacy Without Constant Reenforcement

\section{$\underline{\text { SL Wall }}^{1}$, DL Clarke ${ }^{1}$, NL Allorto ${ }^{2}$}

${ }^{1}$ Greys Hospital, PIETERMARITZBURG, South Africa

2Edendale Hospital, PIETERMARITZBURG, South Africa

Objectives: The aim of this study is to compare doctors' knowledge regarding analgesia in paediatric burns patients in a setting where analgesia protocols are provided but not reinforced to a setting where the same protocols are used but with constant re-enforcement from burns surgeons.

Methods: We reviewed questionnaires completed anonymously by doctors managing burns children in the Pietermaritzburg (PMB) Hospital Complex and the referral hospitals. We evaluated knowledge regarding the use of procedural sedation, including drugs and dosages, assessment of adequacy of analgesia and assessment of pain.

Results: The questionnaire was completed by 43 doctors with $53 \%$ of the participants working in the referral hospitals. Procedural sedation was given by $98 \%$ of doctors. All PMB doctors giving procedural sedation used ketamine compared to $38 \%$ in the referral hospitals, which was statistically significant $(\mathrm{X} 2=18.237 ; \mathrm{p}<0.001)$. Eighty- one percent of PMB doctors were aware of the correct doses of ketamine and compared to $8 \%$ of referral doctors. This was statistically significant $(\mathrm{X} 2=$ 21.778; $\mathrm{p}<0.001$ ). When assessing the adequacy of analgesia, all of the doctors from PMB used a scoring system or clinical impression. In the referral doctor group, $54 \%$ used a scoring system, $38 \%$ used the child screaming as an indicator of inadequate analgesia.

Conclusion: We have identified a discrepancy in knowledge between staff in an academic burn centre and those in peripheral referral hospitals. This discrepancy translates into differences in quality of burn analgesia which patients receive.

P015 Regulation of Aquaporin 3 Expression with Aloe vera based Formulation (Alhydran) in Skin Transplants after Operative Therapy of Deep Burns

IVidmar ${ }^{1}$, R Kavalar'1, U Potocnik ${ }^{2}$

${ }^{1}$ University Medical Centre Maribor, MARIBOR, Slovenia

${ }^{2}$ Faculty of Medicine, University of Maribor, MARIBOR, Slovenia 
Objectives: Problems with dehydration of transplanted skin after operative therapy of deep burns are common in clinical care. Aquaporin 3 (AQP3), one of transmembrane proteins that transport water and small solutes such as glycerol across cell membranes, is abundantly expressed in keratinocytes of mammalian skin epidermis. AQP3 is shown to play a role in the hydration of mammalian skin epidermis and to regulate the metabolism of lipids in skin and the proliferation and differentiation of keratinocytes. While $A$. vera has a long history of use in the topical care of skin burns and other wounds. Its efficacy and mechanisms of action, particularly in skin hydration of transplanted skin after operative therapy of deep burns, have not been reported.

Methods: 5 patients with deep burns were included in the study. After tangential necrectomy of burned tissue, split thickness skin grafting was performed. Following the achievement of skin ingrowth, an A. vera based formulation (Alhydran) was applied twice daily on transplanted skin for 21 days. Punch skin biopsies were harvested from transplanted skin that was treated by Alhydran and from transplanted skin that was not treated by it (control) in the same body area at days $0,1,7,14$ and 21. Expression of AQP3 in skin samples was studied by immunohistochemistry and reverse transcription-polymerase chain reaction (RT-PCR).

Results: Significant upregulated expression of AQP3 was detected in treated transplanted skin compared to controls $(p<0.05)$. There was evidence of increased upregulation of AQP3 with time of application.

Conclusion: Topical treatment of split thickness skin transplants after operative therapy of deep burns with $A$. vera based formulation can improve hydration of transplanted skin through upregulated expression of AQP3. Studies in cell cultures will follow to clarify the mechanism of action.

P016 Staring up a mother child burn camp

T Rose, E Vandermeulen, C Parmentier, P Vanlaeke

Military Hospital, BRUSSELS, Belgium

Objectives: The mother and child burn camp as succesfull start up helping young kids in the after care period

Methods: Last year the after care organization Pinocchio started up the first Mother/Child burn camp. In the past it was not so easy to treat small children in thermal cure camps because they needed the close contact with their parents.

Therefore we had the idea to start a thermal cure camp for mother together with their burned childern.

Results: We want to share our first impressions of this new start up burn care camp

Conclusion: To be continued!

P017 Are we doing enough for burns? Doctors in Dhaka have a say

SI J Chong', T.L. Low ${ }^{1}$, A. Cheah ${ }^{2}$, XY Chong', K.C. Tan², F.C. Liam²

${ }^{1}$ Association for burn injuries Singapore, SINGAPORE, Singapore

${ }^{2}$ Singapore General Hospital, Singapore

Objectives: Burns are a global public health issue particularly in developing countries. This study aims to evaluate the epidemiology of burns in Dhaka.

Methods: A survey form was employed to standardise the interview process. Survey form was prepared in English. Doctors were approached randomly at the local Burns unit at Dhaka, while they were at work. All interviews were conducted, one-to-one, with the assistance of a translator and withthedoctors' verbal consent.

Results: 15 doctorsparticipated in the study. $60 \%$ of them were males while the rest were females. 80\% (12) chose English to be the language medium of choice whereas $13.3 \%$ (2) was other languages and $6.7 \%$ (1) was Bengali. The two most common burn injuries were thermal (15) and electrical burns (15).100\% agreed that thermal burns were more prevalent than electrical burns.Othertypes of burns reported werechemical (9), friction (7), radiation (2), lightning (1) and abuse (1). 100\% of them agreed that there were not enough safety precautions in place to prevent electrical burns. $80 \%$ of them 
thought that education was lacking in the community. Other popular causes reported were inadequate general public awareness $(73 \%, 11)$, overcrowding in poor housing conditions $(67 \%, 10)$, poor education levels $(53 \%, 8)$ and poor city/home layout/hazard placements $(53 \%, 8) .11(73.3 \%)$ thought that doctors, instead of nurses should lead Burns Resuscitation and $9(60 \%)$ thought that doctors, instead of nurses. the complication of Burn Injuries which patients complain the most of was disfigurement.

Conclusion: The high prevalence of burns, undeniably is attributed to anthropogenic reasons. Thus, burns are preventable, excluding that caused bynatural disasters for example in this study, lightning. The consensus among these Dhaka doctors is that much effort is still needed to helpthe burns community.

P018 The Feasibility of Remote Enzymatic Debridement with Nexobrid for Burns

E Lucas

Queen Victoria NHS Foundation Trust, EAST GRINSTEAD, United Kingdom

Objectives: To demonstrate the feasibility of early enzymatic debridement with Nexobrid as an outreach service by the burns team, in patients admitted to acute and remote hospitals with no burns service who are unable to be transferred to a Burns Unit promptly.

Methods: A patient with 40\% TBSA deep-partial/full thickness burns was enzymatically debrided whilst intubated in ITU in an acute hospital with no burns service. Debridement was performed by trained burns staff using a standard protocol for Nexobrid use.

Results: Following successful debridement with Nexobrid, the patient was fully healed within 8 weeks and required no further debridement or skin grafting.

Conclusion: The importance of early burn debridement is recognised as part of the Burns Standard of Care. When burns patients are hospitalised in other specialist units due to complex lifethreatening injuries, it is not always possible or appropriate to perform surgical debridement until the patient is stable enough for transfer. With no onsite burns professionals, debridement is often delayed, resulting in the loss of viable dermis and spontaneous healing. This can lead to potentially unnecessary surgical procedures and poorer outcomes.

We have demonstrated the feasibility of Nexobrid treatment performed as an outreach service by the burns team to enable early wound debridement for a patient whose treatment would have otherwise been delayed, and are working to establish a standardised protocol for patient selection and the delivery of remote enzymatic debridement by trained burns staff. To our knowledge, this is the first time Nexobrid has been successfully used remotely, outside a specialist burns unit. Having demonstrated its success, we believe that this presents an important new treatment option to improve access to prompt burns treatment for a specific patient group who are unable to be transferred to a Burns Centre.

\section{P019 ORGANIZATION AND PROVISION OF MEDICAL CARE FOR BURNED PATIENTS IN THE RUSSIAN FEDERATION}

A. Alekseev $^{1}$, A. Bobrovnikov ${ }^{2}$, N. Malyutina ${ }^{2}$, S. Popov ${ }^{2}$

${ }^{1}$ A.V.Vishnevsky National Medical Research Center of Surgery,, MOSCOW, Russia

${ }^{2}$ Russian Medical Academy of continuous Postgraduate Education, MOSCOW, Russia

Objectives: To present the experience of organizing medical care for burned patients in Russia.

Methods: The results of providing medical care for burned patients in burn centers and departments in Russia, for 2000-2017 years, were analyzed.

Results: To achieve optimal results, the continuity of treatment was important during provision of emergency medical care, qualified care in medical institutions, and specialized care in burn centers and departments.

With a large number of patients with extensive burns (more than 10-15 people), if necessary, we conduct evacuation of them to burn centers located in other regions of the country.

Clinical recomendations for treatment of burned patients envisage the use of: multicomponent therapy in wards of intensive care and resuscitation; extracorporeal treatment methods; rational 
antibacterial and immune therapy; nutritional support; local treatment of wounds including modern wound dressings, early necroectomy, plastic restoration of skin including with application of cultured skin cells etc.; conservative and surgical rehabilitaion.

Conclusion: The developed system of organization and provision of medical care for burned patients, use of modern methods of medical care had improved results of treatment, reduced disability and mortality.

P020 Surgical Safety Checklist in a Burn Unit: Implementation and Results.

C Aguilar-Chalela, JB Ruiz-Padilla

Hospital H+ San Miguel de Allende, SAN MIGUEL DE ALLENDE, Mexico

Objectives: To improve patient safety through the creation and implementation of Specific Process Guidelines for the correct filling of the Surgical Safety Checklist in The Hospital H+ San Miguel de Allende, Burn Unit.

Methods: Experimental, prospective, non-randomized, quantitative research was conducted from 1 to 17 September 2018, at the Hospital H+ San Miguel de Allende, Burn Unit of the Plastic Surgery Service. Correlational design. Convenience sample of 10 healthcare workers at the Operating Room. Held training for the correct filling of the Surgical Safety Checklist, which included Training Workshop and Specific Process Guidelines in a Burn Unit. There were conducted pre-training and post-training evaluations and three case series.

Results: Pre-training assessments showed only $21 \%$ of correct answers, unlike the post-training evaluation where the $79 \%$ was answered correctly. Compliance with the Checklist for Safe Surgery with 21 items was $100 \%$ in the three case series.

Conclusion: Although the sample was small, there were not near misses, adverse or sentinel events reported in burn related surgical process. It is expected, then, that with the implementation of Specific Process Guidelines for the correct filling of the Surgical Safety Checklist, the risks will be reduced and therefore, the adverse and sentinel events in the short, medium and long term.

P021 Introduction of new diagnostic tools in the daily burn care

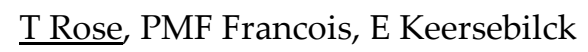

Military Hospital, BRUSSELS, Belgium

Objectives: Optimalisation in diagnostics is the main issue in our daily burn care. Therefore the LDI technique was implanted in our burn care procedure. We want to describe the effects on daily work by using the LDI.

Methods: Description of the use of the LDI in our burn care system.

Results: We will show you the impact of the change in burn care when using the LDI.

Conclusion: We will share our impression after the use of LDI as diagnostic tool, and the pitfalls and benefits concering us.

P022 Accuracy of total burn surface area estimations in Northern Greece.

A Joycey, E Nikolaidou, S Papadopoulou

„G. Papanikolaou, General Hospital of Thessaloniki, THESSALONIKI, Greece

Objectives: In the Greek Health System, General Surgeons provide initial care and, in many cases, conclusive treatment to patients suffering burn injuries, without the obligation to consult a Plastic Surgeon/Burn Specialist. The purpose of this study is to identify how reliable total burn surface area (TBSA) estimations and resuscitation fluids calculations based on these estimations are when made by General Surgeons in Northern Greece.

Methods: Survey data was collected by using a questionnaire based on photographs of 3 patients who were suffering from burns and being treated at our department. Participants were both Plastic Surgeons and General Surgeons employed at hospitals of Northern Greece. We investigated differences in TBSA estimations. We also asked for the amount of the resultant resuscitation fluid volume they thought was needed and investigated how much it deviated from the volume the 
Parkland formula suggests. Finally, we asked for details of the method used to make the TBSA estimations.

Results: We identified both overestimation and underestimation of TBSA. Further to that, resultant fluid volume seems to deviate from what the Parkland formula suggests should be administered.

Conclusion: The above mentioned deviations reveal that patients suffering from burns are not treated appropriately. Our department has taken the initiative of organising educational sessions directed at General Surgeons in Greece, and created a pioneering burns referral system, so anticipating a more reliable TBSA estimation and resulting improved patient care.

P023 Digital picture book to inform children between the ages of 1.5 - 4 years about wound dressing changes

I.K. Zuiker' ${ }^{1}$ L.E. Oosterhoff Karssies', R. Koops van 't Jagt², C. Coenraads ${ }^{3}$, F.T. Everts ${ }^{4}$, M. Klompmaker $^{5}$

${ }^{1}$ Martini Hospital, GRONINGEN, Netherlands

${ }^{2}$ Ruth Koops van 't Jagt, GRONINGEN, Nederland

${ }^{3}$ Het Woeste Woud, GRONINGEN, Nederland

${ }^{4}$ Fret, GRONINGEN, Nederland

${ }^{5}$ Marijke Klompmaker Illustraties, GRONINGEN, Nederland

Objectives: Wound dressing changes are a daily, painful experience, which can be quite overwhelming for young children (1.5 - 4 years old). To inform these young children is a challenge. They have only a small active and limited passive vocabulary. Being informed however, is crucial because knowing what is going to happen and why, may reduce stress. Secondly, being informed provides children with words which facilitates their understanding of what is happening, and a means to communicate with their parents. We aim therefore, to develop a digital picture book to inform these young children and their parents about wound dressing changes.

Methods: The digital picture book was developed by a multidisciplinary team including staff from the burn centre, specifically the child life specialists, an illustrator, producer, communication specialist and narrative health communication researcher. The book can be read to the children by their parents. By including games, sound, and interactivity, the child's attention and curiosity are stimulated.

Results: The digital picture book concerns a story in which the young child is invited to help nurse Zizo (Nurse Alldone) with the wound dressing change of various animals. The interactive elements ensure that the child has an active, leading role in the wound dressing change. The animals have different styles of reaction; scared, resisting, sad. Next, children can select possible coping strategies. In all cases the story supports the animal's behaviour and chosen coping strategies in a positive matter. Thus the story reflects possible reactions and coping strategies of the child, labelling those reactions and strategies positively. An additional story character, the clumsy doctor Dinges (doctor Flippity-Flop) helps making the story funny and accessible.

Conclusion: With this digital picture book we hope to achieve better coping of young children with their wound dressing changes and thus less stress, which will be evaluated subsequently.

Picture 1 : https://www.eventure-online.com/parthenuploads/89/9EBA/add_1_534394_c8c05cd1-1dc3-4fcf-a672-38db79f32810.jpg

Caption 1: scenes from the digital picture book

P024 Management of chemical burns with boiling sulfuric acid solution, in the presence of copper sulphate - case report

\section{A Bordianu}

Bagdasar-Arseni Emergency Hospital Bucharest, BUCHAREST, Romania

Objectives: Copper sulphate is created by treating copper with sulfuric acid. On its own, copper sulfate is mildly toxic, while sulfuric acid mixtures can cause life threatening injuries. We report a 
fatal case of a chemical burn and inhalation injury caused by exposure to boiling sulfuric acid mixture and copper sulphate.

Methods: The patient was a 54 year old man who worked in a factory. He was injured when a sulfuric acid boiler spilled, while mixing the solution with copper to create copper sulphate. Four days following the accident he was transferred to our unit. He had grade IIB and grade III chemical burns over $25 \%$ of his body and severe inhalation injury. He was febrile and anuric, unconscious, intubated, and hemodynamically unstable. He was treated supportively with fluid replacement and vasopressor support. Haemodialysis was performed urgently.

Results: Fourteen hours after admission the patient expired.

Conclusion: Copper sulfate is moderately toxic, and sulfuric acid solutions are corrosive, especially at high temperatures. In high concentrations or long exposures, chemical burns may occur, with widespread fulminant negative effects on internal organs (e.g. liver and kidney failure). Haemodialysis should be applied when acute renal injury is detected, and continued until kidney function is recovered.

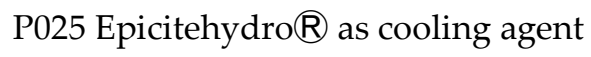

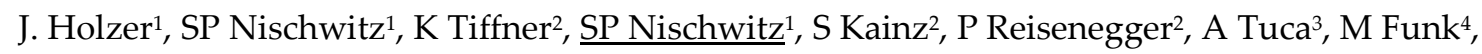
T Birngruber'2, SI Mautner'2, LP Kamolz ${ }^{3}$

${ }^{1}$ Joanneum Research - COREMED, GRAZ, Austria

${ }^{2}$ Joanneum Research - HEALTH, GRAZ, Austria

${ }^{3}$ Medical University of Graz, GRAZ, Austria

${ }^{4}$ QRSkin GmbH, WÜRZBURG, Germany

Objectives: Cooling a burn injury is essential and can lead to less intradermal damage. Plain tap water at moderate temperature for about 20 to 30 minutes is most efficient. Unfortunately, most burn injuries reach the hospital without any pre-clinical cooling, possibly because of the lack of a cooling agent. We investigated if a bacterial nanocellulose (BNC)-based wound dressing containing about $95 \%$ water can cool a burn injury sufficiently and if this cooling effect can reduce the intradermal damage.

Methods: A human skin explant was burned with a heated steel block to induce contact burn injuries, of which half were treated with epicitehydro ${ }^{\circledR}$ and a paraffin gauze dressing. Temperature sensors were placed intradermally measuring the temperature changes in the dermis. At different time points, biopsies were taken for histological assessment.

Results: The results show that the application of epicitehydro® significantly reduced the intradermal temperature after the burn injury. The intradermal measurements show maximum temperature spikes of up to $66^{\circ} \mathrm{C}$ and a rapid decrease after applying the treatment. The area under the curve in the treatment group was $6 \%$ less than the untreated with a $\mathrm{p}<0.05$. The histological assessment showed concordant results with less dermal damage in the treatment group.

Conclusion: The use of a BNC-based wound dressing with a high water content can significantly lower the intradermal temperature after a contact burn and can reduce the thermal damage in the skin. A secondary dressing that allows the water inside the BNC-based wound dressing to evaporate slower additionally prolongs the cooling effect. The use of such a wound dressing could find use in a preclinical setting where water is not ready at hand to cool a burn.

P026 PHMB uptake in epicitehydroß

J. Holzer ${ }^{1}, \underline{\text { SP Nischwitz }}{ }^{1}$, AC Tuca ${ }^{2}$, IB De Mattos ${ }^{3}$, SP Nischwitz ${ }^{1}$, F Groeber-Becker ${ }^{3}$, M Funk ${ }^{4}$, D Popp 5 , SI Mautner ${ }^{6}$, T Birngruber ${ }^{6}$, LP Kamolz ${ }^{2}$

${ }^{1}$ Joanneum Research - COREMED, GRAZ, Austria

${ }^{2}$ Medical University of Graz, GRAZ, Austria

3Fraunhofer Institute for Silicate Research ISC, WÜRZBURG, Germany

${ }^{4}$ QRSkin GmbH, WÜRZBURG, Germany

5Shriner Hospitals for Children, GALVESTON, TX, USA

Joanneum Research - HEALTH, GRAZ, Austria 
Objectives: Burn patients are especially prone to wound infections due to their vast wound areas. Therefore, a need for novel antiseptic wound dressings remains. The loading of bacterial nanocellulose (BNC)-base wound dressings with antiseptics has already been successfully performed but the described procedure is not applicable in a clinical emergency setting. A clinically feasible approach was tested in this study.

Methods: Sheets of epicitehydro ${ }^{\circledR}$ were placed into antiseptic solutions containing PHMB (Prontosan ${ }^{\circledR}$ and LAVANID ${ }^{\circledR} 2$ ) and were left to soak for up to two hours. At different time points, punch biopsies were taken and analysed for their concentrations of PHMB. To test the effectiveness, an agar-diffusion-assay with Staph. aureus was performed.

Results: After 30 minutes, clinically relevant concentrations of PHMB were measured in epicitehydro®. The 30-minute PHMB uptake for Prontosan ${ }^{\circledR}$ and LAVANID® 2 resulted in concentrations of $0.05 \%$ and $0.019 \%$, respectively. The maximum uptake from Prontosan ${ }^{\circledR}$ and LAVANID ${ }^{\circledR} 2$ reached $95 \%$ and $76 \%$, respectively. The agar-diffusion-assay results were concordant.

Conclusion: This experiment showed that the loading of BNC-based wound dressings with PHMB-containing antiseptics can be achieved by a simple and time-saving procedure. A PHMB concentration as low as $0.001 \%$ inhibits all bacterial growth, indicating that the 30 -minutes concentrations of PHMB in epicitehydro® are far higher than the minimal inhibitory concentration.

P027 Continuous $\mathrm{pH}$ monitoring in wounds using a composite indicator dressing - a feasibility study

$\underline{\text { SP Nischwitz }}{ }^{1}$, IB De Mattos², E Hofmann'1, F Groeber-Becker², M Funk ${ }^{3}$, GJ Mohr, LK Branski5, SI Mautner ${ }^{6}$, LP Kamolz ${ }^{7}$, J. Holzer ${ }^{1}$

${ }^{1}$ Joanneum Research - COREMED, GRAZ, Austria

2Fraunhofer Institute for Silicate Research ISC, WÜRZBURG, Germany

${ }^{3}$ QRSkin GmbH, WÜRZBURG, Germany

${ }^{4}$ Joanneum Research - MATERIALS, WEIZ, Austria

${ }^{5}$ Shriner Hospitals for Children, GALVESTON, TX, USA

${ }^{6}$ Joanneum Research - HEALTH, GRAZ, Austria

${ }^{7}$ Medical University of Graz, GRAZ, Austria

Objectives: Modern burn care strives for new means to guarantee optimised wound healing. Several studies have shown a correlation between the $\mathrm{pH}$ value in a (burn) wound and successful wound healing. A multitude of devices to monitor $\mathrm{pH}$ is available, all requiring direct wound contact and removal of the dressing for $\mathrm{pH}$ monitoring. The aim of this feasibility study was to create a sterile and easy to handle method for $\mathrm{pH}$ monitoring while simultaneously using an advanced wound dressing.

Methods: Dressing sheets of biotechnologically generated nanofibrillar cellulose (epicitehydro) were chemically functionalised with the indicator dye GJM-534. $\mathrm{pH}$-donors with increasing $\mathrm{pH}$ were subsequently applied to the created indicator dressing. To investigate temporal resolution and continuous monitoring we used circular $\mathrm{pH}$-donors with different $\mathrm{pH}(7$ and 10) and decreasing diameters that were placed on another dressing sheet. Clinically relevant spatial resolution was checked by a wound bed simulation with small areas $(8 \mathrm{~mm})$ of higher $\mathrm{pH}(10)$ on a field of lower $\mathrm{pH}$ (7) and vice versa.

Results: The indicator dressing showed a gradual colouring from yellow to dark orange with increasing $\mathrm{pH}$ in steps of 0.3 . After conversion of digital pictures to greyscale values, a sigmoidal distribution with a $\mathrm{pK}$ a-value of 8.4 was obtained. A ring-like pattern with alternating colour change corresponding to the $\mathrm{pH}$ was observed in the continuous monitoring experiment and the wound bed simulation delivered excellent local resolution.

Conclusion: Since the $\mathrm{pH}$ of a (burn) wound can have a significant influence on wound healing, a $\mathrm{pH}$ indicator was successfully linked to an advanced, temporary, alloplastic wound dressing material. We were able to show the possibility of $\mathrm{pH}$ monitoring by the dressing itself. Additional testing, including studies with large case numbers for optimisation are necessary before clinical implementation. 
P028 Creation of an interdisciplinary Burn Center log book

D Carel, MM Berger, O Pantet, A Schmidt, S Terrier

Centre Hospitalier Universitaire Vaudois, LAUSANNE, Switzerland

Objectives: Generate a handy pocket document integrating all disciplines and treatments from admission to the burn centre until discharge and rehabilitation

Methods: To address the very different needs of physicians, surgeons and PAM, interdisciplinary meetings were organised. Existing protocols and procedures were collected, and updated. Protocols had been generated for the EBA verification in 2015, but existed as multiple separate documents. Several meetings were organized between 2016 and 2018 to promote the integration of recent literature references (PubMed, Cinhal). Agreement process was by consensus after multiple circulation of documents. They were grouped by phase of treatment and by organ care, more than by specialty which was a novel approach. The final document was again circulated, and sent for printing after validation. An electronic version was kept as the references and to enable future upgrades.

Results: The final document includes 162 pages, multiple figures and photographs, and 53 references. Format is A6, enabling pocket placing. Chapters are : admission, respiratory care, cardiovascular, neurologic, environment, renal, digestive, surgical and topical treatment. The publication generated an intense team enthusiasm. The book enabled a general view of burn care that generated several constructive comments for the future developments.

Conclusion: The book enabled a general view of our practices that gave recognition of every involved specialist. While the creation required heavy work, the result was rewarding and enforced the team feeling. The book is meaningful to the team as to the patient needs.

P029 Infrared thermal imaging utilization in determining tissue viability in electrical burn injury after fasciotomy

\section{GAD Duhita, MEL Putri, AKH Syarif}

Burn Unit, Cipto Mangunkusumo Hospital, Jakarta, JAKARTA, Indonesia

Objectives: Electrical burn injury is one of major causes of disability and mortality in productive age. In 2018 alone, it is among the most frequent case in our centre. Early fasciotomy is the first choice of limb salvage caused by deep muscle necrosis and tissue oedema. In electrical burn injury, there are specific problems among which is to assess tissue viability in order to salvage the viable tissue and remove the devitalized tissue. Rapid assessment of viability is needed to provide proper treatment for these patients. Forward looking infrared (FLIR) is a hand held thermography that might be beneficial to the cause by assessing blood flow via temperature. This article will evaluate the accuracy of FLIR in determining the tissue and muscle vitality after fasciotomy in electrical burn injuries prior to further surgical intervention.

Methods: Patients with electrical burn injury who had undergone emergency fasciotomy of the limbs will be evaluated with thermal imaging handheld camera in the operating theatre. The tissues that were visualized similar as body temperature considered to be viable tissue, and non-viable tissues were removed. We present two cases with electrical burn injury that were managed with the aid of FLIR.

Results: After fasciotomy due to impending compartement syndromes of 2 different patients, tissue viability was evaluated with FLIR. Non-viable tissues were identified with FLIR and were consistent with clinical findings. After non-viable tissue removal was performed in both patients, the tissue removal proved to be adequate and there were no further debridement needed.

Conclusion: FLIR is one of the tools that will help surgeons in determining viability of tissues after fasciotomy in burn injuries in order to decide whether to salvage extremities or performed amputations. In the future, it may become the standard tools in burn injury assessment.

P030 Considerations for the development of an App to measure burn patient outcomes: an international IT perspective 


\section{T Klotz, R Kurmis}

Royal Adelaide Hospital, ADELAIDE, Australia

Objectives: Technology is increasingly utilised to improve and streamline patient care. Electronic medical record systems and hand held smart devices are increasing, as is the use of applications (Apps). In our endeavours to digitise a manual scar assessment tool previously developed at our centre, we uncovered many important considerations unique to this rapidly evolving space.

Methods: A needs assessment was conducted prior to project initiation. The optimal digital platform was determined, based on accessibility, ongoing App maintenance requirements, and subsequent costs. An experienced health Apps designer was consulted. Project funding was sourced via grant application. Local ethics and research governance consent was obtained for the reliability testing on patients, along with the use of de-identified and consented clinical photographs within the App itself.

Following project initiation, local information computer and technology (ICT) security requirements changed along with implementation of European General Data Protection Regulations (GDPR), leading to changes in the App design relating to patient data input and output capabilities. For launching within the US the Health Insurance Portability and Accountability Act (HIPPA) requirements were also considered. The App disclaimer was reviewed by health department legal experts. Inter-rater reliability testing was conducted with the guidance of a statistician.

Results: A scar assessment App the ClinMAPSTMPro was developed that complies with regulations pertaining to safety of sensitive individual data. The App assists clinicians and researchers with assessment of scars with a reporting output suitable for inclusion in paper or electronic medical records.

Conclusion: Smart devices are increasing, supporting clinical decision making. Due to rapidly changing requirements and various international regulations governing the collection, use and storage of patient information, a considered approach is needed prior to development of Apps for use in burn injury care.

Local ICT security, GDPR and HIPPA requirements should be considered when implementing App based assessment tools into clinical practice.

P031 'Nothing about us, without us' - How to involve burn survivors in research?

J Hiddingh $^{1}, \underline{\text { M. Akkerman }}{ }^{2}$, MK Nieuwenhuis ${ }^{3}$

${ }^{1 B}$ urn Centre Groningen, Martini Hospital, GRONINGEN, Netherlands

${ }^{2}$ Association of Dutch Burn Centres, Burn Centre Groningen, GRONINGEN, Netherlands

${ }^{3}$ Association of Dutch Burn Centres, Burn Centre Groningen, Martini Hospital, GRONINGEN, Netherlands

Objectives: In the current health care system, patients are gaining a more active role. This patientcentered approach to medicine allows patients to be involved in their own medical decisions, which is also true in current burn care. As research aims to inform and optimize burn care and rehabilitation, we consider it essential to actively involve patients in burn research as well.

Methods: From their personal experiences, burn survivors can offer a unique point of view which can be relevant in different phases of the research process, for instance preparation, execution, communication, evaluation, and, if possible, implementation. Many opportunities exist for burn survivors and/or their representatives (e.g. parents, partners, informal caregivers) to participate actively in the entire research process. From the literature, it is advised to involve them as early as possible, preferably from the start.

Results: Active involvement of burn survivors in research can, for example, lead to more relevant and suitable research questions, improved readability of patient information and informed consent documents, relevant measurement instruments, improved recruitment and response rates, enriched data analyses, and improved communication of results towards (other) burn survivors. The extent of involvement can vary from being informed (e.g. newsletters, research presentations), to providing information (sharing experiences), providing advice and feedback, or even co-operating actively. Before burn survivors are asked to be involved, several questions have to be addressed. What do we 
aim to achieve with active involvement of burn survivors? Which knowledge is required from them (education, communication skills, experiences)? Which (financial) support can be offered? Furthermore, for successful collaboration it is really important to clearly inform burn survivors about their involvement and discuss mutual expectations.

Conclusion: Until now, burn survivors were primarily actively involved in prioritizing burn research and judging grant applications. Now, we started to involve burn survivors from start to finish in our research.

P032 Combination therapy using nonablative fractional laser and intralesional triamcinolone injection for hypertrophic scars and keloids treatment

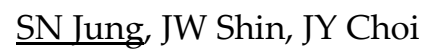

UIJEONGBU sT. Mary's hospital, college of medicine, The catholic university, UIJEONGBU, South Korea

Objectives: For intractable hypertrophic scar, multimodal treatment is a promising strategy. We investigated the clinical efficacy of combination therapy of intralesional injection (ILI) and nonablative fractional LASER (NAFL), compared to ILI therapy alone.

Methods: Total of 57 patients with hypertrophic scars or keloids were enrolled. Total of 19 patients were excluded due to age and medical comorbidities. ILI monotherapy group $(n=21)$ and ILI plus NAFL group $(n=17)$ were evaluated. ILI was performed using1:1 Triamcinolone with lidocaine with $0.1 \mathrm{cc} / \mathrm{cm}^{2}$. NAFL was performed using $1550 \mathrm{~nm}$, erbium-glass fractional LASER with $50 \mathrm{~mJ} x$ $40 \mathrm{spot} / \mathrm{cm}^{2}$. All treatments were 4 weeks apart.The outcome was evaluated with 1) number of treatment sessions until treatment endpoint, and 2) Patient and Observer Scar Assessment Scale (POSAS). Recurrence or treatment failure was defined as no improvement after 6 months of treatment completion. The data was analyzed with independent sample t-test and recurrence rate was assessed with chi-square test.

Results: Mean number of treatment sessions showed statistical significance (6.95 in control group vs 5.47 in combined therapy group, $p=0.042$ ). Baseline patients scale (PS) did not show significance ( 38.38 versus $36.18, \mathrm{p}=0.580$ ). However, statistical significance was found between two groups (23.77 versus 13.36, $\mathrm{p}=0.0004)$. Baseline mean observer scale (OS) were not significant $(42.08$ versus 38.1, $\mathrm{p}=0.302$ ). At six months post therapy, Mean OS showed significance (24.15 versus 17.64, $\mathrm{p}=0.046)$. Total scale (PS + OS) showed no difference at baseline ( 24.15 versus $17.64, \mathrm{p}=0.347)$, but showed significant difference at six months post therapy ( 47.92 versus $31.00, \mathrm{p}=0.002)$. The recurrence rate was $38.1 \%$ in control group and $35.3 \%$ in combined therapy group $(\mathrm{p}=0.859)$.

Conclusion: Combined therapy with fractional laser and steroid intralesional injections showed significant improvement of scar quality than control group. Ideal laser parameters and treatment sequence should be established with further investigation.

P033 Using 2 diagnostics tools to estimate the depth of burns

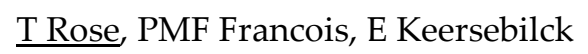

Military Hospital, BRUSSELS, Belgium

Objectives: Today we use 2 diagnostic tools in our daily burn care practice: The LDI technique and the Hyperspectral camera.

The information got from those diagnostic tools is used to estimate the depth of a burn wound.

Methods: We use both diagnostic tools as much for all our patients. The data is used to became an objective healing information of the burn wound.

Results: We will share our first results while using the both diagnostic tools.

Conclusion: The advantage of having both techniques available in our burn unit give us the opportunity to compare the use and the results of both techniques.

P035 The Impact of an International Educational Program on Transforming Practice and Outcomes in Scar Management: Are there Regional Differences? 
VG Gut $^{1}$, PM Moortgat ${ }^{2}$, KM Maertens², RF Fanstone ${ }^{3}$

${ }^{1}$ University Hospital Zurich, ZURICH, Switzerland

2OSCARE, ANTWERP, Belgium

${ }^{3}$ Swansea University Medical School, SWANSEA, United Kingdom

Objectives: Scar Academy is an educational program for healthcare-professionals dedicated to the understanding and treatment of (burn)-scar. Scar Academy was developed by Oscare\#_ftn1 in 2010 with the aim of transforming practices and outcomes in scar-management. Since its founding Scar Academy has organized several events in Belgium, United Kingdom (UK) and Germany, Austria and Switzerland (DACH).

After every course participants had the opportunity to evaluate the event. The aim of this study was to compare the effects of an international educational program on scar-management between 3 European regions.

[1] Oscare, Organisation for burns, scar after-care and research, Antwerp, Belgium

Methods: To date over 1000 people attended one or more Scar Academy events (43\% PT-OT, 28\% Doctors, $19 \%$ Scientists and 10\% Nurses). Lectures and workshops on a wide variety of scar-related subjects were delivered by an international faculty. Survey results included an online pre-course questionnaire and a post-course evaluation.

Results: The response rates were $87 \%$ for the UK, $85 \%$ for Belgium and $75 \%$ for the DACH. Results indicate that Scar Academy did deliver new knowledge and improved the participants trust in their newly acquired abilities (UK $+23 \%$, DACH $+37 \%$, Belgium $+39 \%$ ). After the course $65 \%$ of the participants (UK 60\%, DACH 72\%, Belgium 63\%) changed their subjective and objective scarassessment methods for clinical practice and research. The knowledge of the participants about the various treatment options was greatly expanded by the course, which led to the implementation of new treatments. Scar Academy was extremely well received, 87\% of participants (UK 98\%, DACH 75\%, Belgium 90\%) would recommend Scar Academy to a colleague. Integration of skills workshops could offer more potential for practical training

Conclusion: When a relevant, high-quality international course is delivered to the right audience in collaboration with local partners the impact can be highly effective and efficient, even in different European regions.

P036 Thermal Imaging Device as an Aid to Determine Burn Wound Depth: A Case Series

D Kurniasari, AN Syarif, RA Wardhana

Cipto Mangunkusumo Hospital, Medical Faculty, Universitas Indonesia, JAKARTA, Indonesia

Objectives: The aim of this study is to know the use of thermal imaging device to determine burn wound depth

Methods: We did clinical and thermography assessment using Flir One ${ }^{\circledR}$ thermal imager to determine the conversion of burn depth of patient before \& after the excision and after the skin grafting. Subsequently, temperature differences between the burn wound and healthy skin $(\Delta \mathrm{T})$ were measure and compared before and after the excision.

Results: We performed clinical and thermography assessment in 3 patients, 2 male and 1 female patient, with age range 25-48 years old, with burn size 7.5\% - 41\% TBSA due to flame and scald. Two patients showed low temperature (temperature of burn area: $34.2^{\circ} \mathrm{C}, \Delta \mathrm{T}:-2.2^{\circ} \mathrm{C}$ ) on the area of the burn wound so we decided to excised it. After the surgery it shows that the temperature increase (temperature of burn area: $32.7^{\circ} \mathrm{C}, \Delta \mathrm{T}:-0.5^{\circ} \mathrm{C}$ ), that indicated the improvement of the circulation. One patient showed an area of burns with an average temperature (temperature of burn area: $36.9^{\circ} \mathrm{C}, \Delta \mathrm{T}$ : $2.2^{\circ} \mathrm{C}$ ). We decided not to excise and appear epithelialization on the $7^{\text {th }}$ day post burn.

Conclusion: Thermography assessment such as Flir One ${ }^{\circledR}$ thermal imager can be utilized to determine the depth of burns in addition to clinical assessment. It benefits not only for the functional purpose, but also affordable price and practically used. In the future it may be useful to help both experienced surgeons and novices. 
P037 Toxic Epidermal Necrolysis and Epidermolysis Bullosa successfully treated with cultured skin grafts EPIFAST

\section{AMS MASSE SANCHEZ}

MEXICAN SOCIAL SECURITY INSTITUTE, GUSTAVO A. MADERO, Mexico

Objectives: To seek an appropriate treatment for patients with Toxic Epidermal Necrolysis (TEN) and Epidermolysis bullosa (EB) since there are no guidelines for it, and the patients behave as burned ones. Therefore, cultured skin grafts "EPIFAST" were used.

Methods: (1) A 3 years old female developed an adverse reaction to medication, after $48 \mathrm{hrs}$, a Toxic Epidermal Necrolysis (TEN) was developed in $80 \%$ of the body surface (skin). (2) A 6 years of old male with Convulsive crisis, which makes allergy to Lamotrigina at 7 days, TEN was developed in $75 \%$ of the body surface, meriting intensive care management. (3) Female 3 years old with Epidermolysis Bullosa on different parts of the body and infection.

Results: On the 1st day of treatment, Epifast was applied to 3 patients (50\% of the lesions to patients with NET and $100 \%$ to NB) were removed one week later, and Epifast was applied in the other $50 \%$ of the lesion (NET), the patient with EB healed with a single application of Epifast.

Conclusion: The surgical technique applied with cultured skin grafts EPIFAST, demonstrated better results than expected, since adequate skin cover throughout the treated surface was achieved. It stimulates the healing and shortened the recovery period of the patient.

P038 Comparsion of traditional and modern dressing methods on children with deep burn injury after autologous transplant

JG Jozsa, VF Varga, JZS Juhasz

Department of Pediatrics, Surgical Unit, PÉCS, Hungary

Objectives: Comparison of the postoperative treatment of the patients who were transplanted because of deep (II/2, III degree) burn injurys.

Methods: 16 transplantation were performed because of deep burn injury in the Pediatric Surgery ward between Janury 1st 2015. and Decembre 31th 2017. Two groups were developed for the retrospective study. In Group 1 Grassolind mesh with Betadine solution, while in Group 2 Aquacel Ag foam dressing with Curiosa gel were applied to cover the transplanted skin.

Results: In Group 17 children, while in Group 29 children were involved in the clinical study. The average age was under 5 years in both groups.

The cause of the injury was scalding with hot liquids in all cases in Group 1 . The extent of the burn in three cases was $5-10 \%$. The children spent average 21.9 days in hospital, meanwhile average six anesthesia were performed to change the bandage. The removal of the bandage usually happened on the 13th day.

4 children were injured because of scalding, while 5 children suffered from contact burns in Group 2. The extent of the injury in four cases was less than $5 \%$, in two cases was $5-10 \%$, and in three cases was more than $10 \%$. The children spent average 13.8 days in the hospital, meanwhile average three anesthesia were performed on them. The removal of the bandage usually happened on the 10th day.

Conclusion: In case of a II/2, III degree deep burn injury, after the required transplantation and the use of the Aquacel Ag foam dressing and Curiosa gel resulted in significant decrease of the number of performed anaesthesias and the days spent in the hospital. In Group 2, where the intelligent bandage was used, the final removal happened 3 days earlier.

P039 Treatment of partial thickness facial burn injuries in children with zinc-hyaluronic gel.

IG Jozsa, VF Varga, JZS Juhasz

Department of Pediatrics, Surgical Unit, PÉCS, Hungary

Objectives: Burns is a common type of traumatic injury in childhood. Nowadays, several treatment options are available to treat the second-degree facial burns conservatively.

Methods: Burns is a common type of traumatic injury in childhood. Nowadays, several treatment options are available to treat the second-degree facial burns conservatively. 
Results: The average age were under 5 years in our study. 10 children were injured because of scalding, while 5 children suffered from contact burns. The extent of the injury in all cases was less than $5 \%$.

None of the 15 children treated with this gel were diagnosed with wound infection. The authors observed the epithelialization of the burned areas on the $7-8^{\text {th }}$ day after primary conservative treatment. The gel efficiently promotes epithelialization in all cases. Further advantage of Znhyaluronic gel is to enhance cell regeneration and antibacterial effects.

Conclusion: Based on the authors' experience, with this gel, a gentle, child-friendly, costeffective treatment and excellent wound healing observed with favourable cosmetic results.

\section{P040 Effective laser-therapy of post-burn scars}

H Klosova, B Zalesak

University Hospital Olomouc, OLOMOUC, Czech Republic

Objectives: Hypertrophic burn scars produce significant morbidity, they are functionally insufficient, hypertonic, contracting and causing restriction of movement in joints, flexure regions and body orifices. If scar treatment is to be successful, it must be comprehensive. Lasers to treat scars are most commonly used Vbeam laser, Erbium-YAG and CO2 laser. The primary objectives of laser therapy are to supress scars activity - inflammation, hyperemia and edema, to reduce hypertrophy and to release incipient and mild contractures.

Methods: Retrospective analysis of eight-years clinical experience with Erb-YAG, CO2 and Vbeam laser therapy and treatment outcomes of hypertrophy post-burn scars.

Results: Vbeam laser therapy facilitate spontaneous scar regression and supresses immature scar activity - reduces inflammation, hyperemia and edema. Erb-YAG or CO2 fractional ablative resurfacing significantly reduces height of more mature scars to the level of surrounding healthy skin and reduces the tension of contractioning scars, which leads to release of incipient and mild contractures.

Conclusion: Vbeam, Erbium-YAG and CO2 laser therapy is the effective tool for treatment of hypertrophic post-burn scars and it is desirable to be an integral part of comprehensive scars treatment. Best results are achieved by combined vascular laser and fractional Erbium-YAG or CO2 laser scar revision treatment.

\section{P041 EVALUATION OF PROCALCITONIN ACCURACY FOR THE DISTINCTION BETWEEN GRAM-NEGATIVE AND GRAM-POSITIVE BACTERIAL SEPSIS IN BURN PATIENTS}

J.C. Baltazar Ferreira, C. S. Brandão, A. R. Meireles, I.L. Cabral

Centro Hospitalar e Universitário de Coimbra, COIMBRA, Portugal

Objectives: Sepsis is the main cause of death in burns. Early institution of antimicrobial therapy is crucial to optimize outcomes but superfluous therapy increases adverse events, microbial resistance, and costs.

Blood cultures are the gold standard for diagnosis but can take 48 to 72 hours.

Biomarkers are used to help sepsis diagnosis and distinction between Gram-negative and Grampositive bacterial cause. The aim of this work is to evaluate PCT accuracy for this distinction in burn patients

Methods: A retrospective observational study of adult septic burn patients was elaborated, accounting patients with $\geq 15 \%$ total burn surface area admitted from January 2011 to December 2014 at Coimbra's Burn Unit in Portugal.

Results: The statistical analysis of PCT levels on the first day of microbiological identification in blood samples in this sample of extensively burned patients confirmed previous reports demonstrating significantly higher values in the presence of Gram-negative bacteria comparing with controls or patients with Gram-positive sepsis.

Conclusion: To the authors' knowledge, the present work, with 189 septic burn patients, corresponds to the largest sample already analyzed in medical literature regarding this subject. 
Very high PCT levels (above 3.00 or $5.00 \mathrm{ng} / \mathrm{ml}$ ) would usually be more associated with Gramnegative sepsis, with fair positive predictive value and negative predictive value, and good specificity. In the great majority of the cases, PCT values under $0.5 \mathrm{ng} / \mathrm{ml}$ would not correspond to Gram-negative infections but to Gram-positive or fungal ones.

This retrospective study consistently showed the presence higher PCT levels in burn patients with Gram-negative sepsis, suggesting that PCT, while not precise, may help clinicians in the choice of the empirical antimicrobial therapy, while the definitive, gold standard, microbiological culture results and sensibility tests are not yet available.

Picture $\quad 1: \quad$ https://www.eventure-online.com/parthenuploads/89/9EBA/add_1_546629_e11bbf31-5d06-4f04-8671-c245bd3b6064.jpg

P043 Electronic micro-needling on mature burn scars: a case series report

\section{M.E Peters}

Maasstad hospital, ROTTERDAM, Netherlands

Objectives: The main objective of this report is to investigate and discuss the effects of an electronic micro-needling-device on several characteristics of mature burn scars.

Methods: Skin elasticity was examined with Cutometer ${ }^{\circledR}$, colour was assessed with a Skin Colorimeter ${ }^{\circledR}$ and scar thickness and -density with a $22 \mathrm{MHz}$ High-Frequency Ultrasound device. A clinical assessment of the scar by both the patient (POSAS-P) and the observer (POSAS-O) was obtained by the POSAS. In total 5 burn patients were treated twice with the electronic micro-needlingdevice. The patients were tested before the treatment, after two months just before the second treatment and after five months. For statistical analysis, the Friedman Test was used to determine statistically significant differences over time.

Results: The mean age of the patients was 47 years $( \pm 9.5 \mathrm{y})$ and scars were on average 49 months old $( \pm 27,9 \mathrm{~m})$. The results showed a significant improvement $(\mathrm{p}<.05)$ over time for itch, pliability, thickness and texture assessed by the patient and for thickness, pliability, texture and global score assessed by the observer. For the objective measurements, a statistically significant improvement over time was obtained for elasticity, measured with the Cutometer ${ }^{\circledR}(\mathrm{p}=.022)$ and thickness, assessed with high-frequency ultrasound $(\mathrm{p}=.041)$. No improvement could be recorded for objective colour measurements.

Conclusion: Electronic micro-needling can give added value to the non-surgical treatment of hypertrophic, irregularly shaped, mature burn scars. In the future, a comparison with a control group and a bigger sample size are necessary. An objective measurement of the scar texture and a distinction between specific indications are also desirable.

Picture 1: https://www.eventure-online.com/parthen-uploads/89/9EBA/add_1_554756_3d64afe4b2be-4d8f-922f-431e9d97c564.jpg

Caption 1: Figure 1

P044 The use of VR at the burn hospital MHKA

E Bossuyt

MHKA, NEDER-OVER-HEEMBEEK, Belgium

Objectives: Studies suggest that VR technology can improve quality of care and patients comforts by relieving pain and relaxing patients during their burn wound dressing changes. This pain relief and relaxation is obtained either by distraction, or by the hypnotic and relaxing effect of VR Technology.

Methods: Our VR Kit contains one cellphone, one pair of VR glasses and a headphone. The use of this VR kit is at the decision of the nurse, but the patient is free to decline it.

The nurse needs to gain the trust of the patient, make him aware of his presence and explain every action he makes. The patient need to be older than six years. Contra indication are patients with infections, head trauma, facial wounds, epilepsy, autism, claustrophobia, heavy psychiatric disorders and lice. An evaluation form need to be completed after every use. 
A poster will make patients aware of the possibility to use VR technology during their dressing changes. This way we try to increase the frequency of utilisation of the VR technology

Results: The use of the VR kit is still limited but shows promising results.

Conclusion: Studies did show promising results, but our own critical view on this matter is important. Explaining, installing and registration of the use of the VR kit can take some time. It is hard to predict the level of distraction. This means that the nurses must evaluate the pain/ stress score during each care. It gives the nurses the possibility to clean the wounds more thoroughly with less pain relief medication.

To increase the frequencies of utilisation, patients will need to be aware of this technology. Nurses needed to be habituated with this technology.

P045 The importance of education in Laser Doppler imaging diagnostics

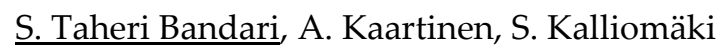

Helsinki University Central Hospital, ESPOO, Finland

Objectives: Laser Doppler Imaging (LDi) has been used in burn depth evaluation since 1993. The purpose of this work is to highlight the factors that are essential in producing reliable results in LDi measurement. After proper training, nurses can perform appropriately LDi imaging. The LDi results are interpreted together with the burn nurse and the burn physician who then decides on the further treatment of the patient.

Methods: The interfering factors affecting the quality of LD imaging and interpretation and reliability of results were identified through literature review. Then, a patient case with two separate imaging sessions were prepared. In the first imaging, manufacturer's instructions and recommendations from the literature review were taken into consideration when performing LD imaging. In the second imaging all the disturbance factors affecting the reliable description of the result were not considered. Then we compared the two different methods.

Results: The presented patient case illustrated, that performing LDi imaging without proper training gives false results. Scientific studies confirm the result of the patient case.

Conclusion: In the evaluation of the depth of a burn, LD imaging is a useful tool when performed by a nurse who has received device specific training.

P046 Challenges to become a competent burn nurse in Helsinki Burn Centre

MELINA Gigante-Bounid, KARITA Niskanen

Helsinki University Central Hospital, ESPOO, Finland

Objectives: In January 2016, Helsinki Burn Centre moved to a new location and unified with a previously existing general ICU. After the unification, the job description of nurses became more diverse. The new unit has an ICU for burn and general patients, a burn ward and an outpatient clinic. Our goal is to present challenges in the process to become a competent burn nurse.

Methods: Our method is empirical, describing our observations and experiences of working as a nurse in our unit and comparing them to clinical studies. The diversity of nurses' job description increase the challenges to achieve competency in burn nursing. In addition to the demanding basic tasks, we have five nursing specialty programs in our unit: burn nursing, dialysis, medical emergency team, shift manager and outpatient clinic. Nurses must manage the basic tasks before entering the specialty programs.

Results: According to clinical studies, it takes approximately 2-3 years to become competent in critical care nursing alone. In our experience, considering the versatility of our unit and the different patient groups treated, the wide range of competency requirements and the large number of nursing staff as well as the turnover of nurses, it takes more time than is shown in studies to become a competent burn nurse in an institution like ours.

Conclusion: The process to achieve competence in burn nursing has been slow for new nurses or nurses before working only in general ICU. Despite the challenges of the new unit in the past three years, we have managed to start the orientation to the specialty program for new burn nurses. In the 
future it will be crucial to further invest in the orientation program of burn nursing to strengthen the nurses' expertise in this field.

P047 Life Music therapy in the burn unit

T Rose, PMF Francois, W Wittenberg

Military Hospital, BRUSSELS, Belgium

Objectives: Life Music used as treatment in a burn unit is recently started in our department

Methods: We will show you the project and share our ideas introducing the use of this therapy item

Results: First results will be shown on site but the start took place 2 weeks ago.

Conclusion: We think that Life Music can be a benefit for the burn patient while beeing hospitalized

P048 Nursing Management in Patients with Vasculer Access (Peripheral Intravenous Catheterization) for Burns

F.S Sahin

Health Sciences University Kartal Dr.Lutfi Kirdar Training and Research Hospital, ISTANBUL, Turkey

Objectives: Nursing management is important in cases with severe peripheral intravenous catheterization due to burns. Factors such as stress caused by previous trauma, anxiety of the relatives of the patients, the characteristics of the pediatric case and the excess of the dressing area have a negative effect on the presence of suitable veins

Methods: In our Wound and Burns Treatment Center, 60 patients under 3 years of age and treated were examined; 40 patients were successfully opened by our peripheral intravenous catheterization nurses.

Central venous catheter was inserted in 15 patients. 5 patients were opened with the support of nurses working in the Children's Department.Burn Due to the existing burns in pediatric and pediatric cases, almost all body areas are covered with dressing by dressing, and there are difficulties due to the fact that there is very little area of the burn area and it is not available in these areas.

Results: It is even more difficult to open the cannula by reasons such as the higher fear and stress experienced in burned child and infant cases, the occurrence as an unusual situation, the high number of unknowns, and the anxiety of the family and relatives.

Conclusion: It is inevitable that the incision of forced peripheral percutaneous intravenous catheterization is inevitable. The number of experienced burn nurses can be planned to be increased and certified.

Picture 1: https://www.eventure-online.com/parthen-uploads/89/9EBA/add_1_540893_b1202c567616-4d00-904e-ed9a6a6c3b61.jpg

P049 Indirect Calorimetry, Non-Invasive Measurements in Practice

J Dimander

Uppsala University hospital, Uppsala, Sweden

Objectives: To describe and share experience from using indirect calorimetry, non-invasive measurements, in a Burn center.

Methods: Calculation of energy requirements from equations are known to have errors compared to measured resting energy expenditure (REE). Therefore, indirect calorimetry is considered gold standard for assessing energy requirement in (burn) patients.

The use of indirect calorimetry at least once a week is recommended for all patients with a TBSA $>20 \%$ in one Burn center. Indirect calorimetry can be measured invasively or non-invasively. Noninvasive measurement is done using a hood. REE is measured during 30 minutes in the morning after at least four hours fasting. The patient should be without extra oxygen and be able to lay still during measurement. This makes it difficult to perform in anxious patients and those in pain. Since non- 
invasive indirect calorimetry measurements represent REE for 30 minutes an assessment of physical activity must be added to the measured value to estimate the total energy requirement. The physical activity level can be assumed to be at least 1.2 for patients in bed but must be increased for patients with higher physical activity.

Results: In a Burn center, strategies identified to make it easier to use non-invasive indirect calorimetry on a regular basis are:

- local recommendations on how and when to use indirect calorimetry

- trained personnel

- decision on when to measure indirect calorimetry during rounds documented in the patients' records and on patients' activity board at the ward

- prepare patient the day before measurement

- measurement early in the morning

Conclusion: Indirect calorimetry, non-invasive measurements can be difficult but are possible to perform on a regular basis in a Burn center.

P050 Nursing Treatment Procedure in Burn Intensive Care of Ten Patients: Case Report

$\underline{\text { F.S Sahin }}$

Health Sciences University Kartal Dr.Lutfi Kirdar Training and Research Hospital, ISTANBUL, Turkey

Results: The bed linen was ironed daily. In this case, the patient's pain expression was constantly evaluated. Before applying interventional procedures, sedative effective analgesic medicines were made and oxygen was given to the patient with a mask.

IV paracetamol medication was administered when necessary.

This way, pain management continued until the patient was transferred from the intensive care unit. On the 12th day of admission to the burn intensive care unit, the patient was transferred to the burn ward.

Conclusion: Careful wound care and pain management forms the basis of care and treatment of patients with TEN. There is no standard care protocol for ulcerations and lesions in the body caused by TEN disease. Nevertheless, under the guidance of the nursing model, patient-centered, quality, multidisciplinary and blood-based nursing care interventions and systemic infections are prevented and the disease is improved without life threatining complications.

Picture 1: https://www.eventure-online.com/parthen-uploads/89/9EBA/add_1_541433_241d0cc2e4b1-4643-ac38-496fc6b1e0a2.jpg

P051 How to best implement a new burn resuscitation strategy in the nurse team?

D Carel, O Pantet, MM Berger, A Schmidt

Centre Hospitalier Universitaire Vaudois, LAUSANNE, Switzerland

Objectives: Having observed that fluid resuscitation was not systematically carried out with feedback loops in case of insufficient or to important fluid administration, the medical team proposed a new resuscitation strategy adapted to patients with $20-50 \%$ or to $>50 \%$ BSA burns. The aim was to delegate a part of the protocol to the nurses. As written, the physicians' algorithm was not understood by the nurses. The implementation process aimed to integrate the nurses.

Methods: Nurse-meetings with the physicians to understand and analyse what scared the nurses. Identification of types of goals that would be acceptable to the nurses mainly urine output and mean arterial pressure (MAP). Detailing the volume rate calculations and generating an XLS file with the equations providing guidance to increases or decreases of hourly fluid resuscitation volumes by +/25\%: definition of cut-offs of urine output, MAP, SvO2, and Lactate that would generate calling the physician. Testing the procedure on simulated cases, and thereafter on 3 consecutive patients.

Results: The equation guided fluid handling was functional and efficient. Over resuscitation was avoided in 3 patients (mean: Age 46y, TBSA 36\%), total volume remained < the theoretical Parkland volume. The number of fluid adaptations varied between 3 and 6 times in $24 \mathrm{hrs}$. The new handling surprised the physicians; nurses and physicians felt a little unsecure about this new procedure. The 
volume to administer the next $24 \mathrm{hrs}$ was not clearly defined, but was $30-40 \%$ below the total of first 24hrs.

Conclusion: While the nurses felt empowered by the new protocol, they felt stressed by this new responsibility. But with the right tools and information, resuscitation was efficient reducing the risk of overresuscitation.

P052 Evaluation of formulation of silicon as alternatives to silicone sheet in post-burn scarring in children

\section{Shah, K Keating}

RMCH, MANCHESTER, United Kingdom

Objectives: Patient/carer evaluation of ointment and spray formulations of silicone gel in children with post-burn scarring.

Methods: Patients attending scar management clinic between Nov 2013 and February 2015 were invited to evaluate the formulations of silicone therapy if they had not previously used any silicone therapy and did not have any allergies or skin conditions precluding the use of silicone. Patients evaluated the ease of use, the drying time of silicone and immediate changes in patients' symptoms and characteristics of the scars by completing a proforma 5 times over a 8 week period.

Results: None of the patients complied with the spray formulation they felt very cold. 25 patients/carers evaluated the silicone gel ointment formulation. 5 patients reported the gel drying time was slow throughout the evaluation, whilst 11 reported this to be fast throughout and 1 patient reporting the drying time to improve with use. 1 parent reported finding it better to apply a thick layer for the sake of the scar but found this increased the drying time. 1 patient reported reducing the use of the gel to 2-3 times per week was better as regular use lead to irritation of the skin. Most parents found it easy to apply although some parents did mention that it depended on the child's behaviour (wriggle factor). Parents felt there was improvement in itching, texture and redness. We identified the need to improve our communication of instructions on use of silicone gel ointment.

Conclusion: Silicone gel (ointment) was found to be useful as an alternative to silicone sheets but not the spray formulation. A randomised control trial to compare the efficacy of the ointment formulation versus the gel sheets in paediatric post-burn scar management would be a valuable study.

\section{P053 BURN WOUNDS MANAGED BY NURSES IN A WOUND CARE CLINIC OF MEXICO, USING EPIDERMAL CULTURED ALLOGRAFTS.}

\section{PI Vazquez, N Fonseca}

Iztapalapa's General Hospital, MEXICO CITY, Mexico

Objectives: To show clinical results obtained by nursing team at the wound care clinic of Iztapalapa's General Hospital "Dr. Juan Ramón de la Fuente", of burn wounds with the use of an epidermal cultured allograft as temporary coverage.

Methods: This is a retrospective review of the cases treated during 2016 by evaluating clinical results based on Vancouver scar scale parameters.

Results: A total of 68 cases were analyzed, divided in superficial second degree, deep second degree and third degree. Average results were: Pigmentation ( $<1)$, Pliability (1), Height (0), Vascularity $(<1)$, Pain (0), Pruritus (1). Number of applications of the allograft (2)

Conclusion: Epidermal cultured allograft has demonstrated to be an excellent option as temporary burn wound coverage, due to immediate and long term clinical outcomes, as well as its easy handling outside the operating room which favors outpatient management.

P054 Impact of immediate enzymatic debridement on survival rate in burn injured patients: experience of BICU, Villa Scassi Hospital, Genoa, Italy

P. Panarese, P. Barabino, L. Bonifazio, E. Grosso, G. Robello, G. Perniciaro

Villa Scassi Hospital Asl3 Genovese, GENOVA, Italy

Objectives: Among various factors that affect prognosis of the burn injured patients, timing of first 
debridement of burn eschar is strongly advocated but rarely studied.

The aim of this study was to evaluate the impact of immediate enzymatic debridement on the burn patient's course and outcome

At our Institution during 2016 we performed enzymatic debridement with Nexobrid on critically ill burned patients (TBSA $>20 \%$ ) in the first 24 hours after injury, mostly on admission in the intensive care unit.

Methods: In order to analyze the impact on survival rate we compared datas of the Nexobrid cohort with datas from previous years, particularly 2014 and 2015 in which we used traditional surgical approach (standard of care-SOC) of tangential excision and autografting.

Patients were divided into subgroups depending on the burn extent: $20-39 \%, 40-59 \%$ and $60-70 \%$ TBSA.

Datas of co-morbidities and inhalation injures have been collected

Results: We matched datas from 31 patients treated with immediate enzymatic debridement with datas from 76 patients treated in the previous years (2014-2015) with traditional surgery (the SOC group). Mean age, gender, co morbidities and inhalation injures were similar in both groups.

The overall mortality was $6.45 \%$ in the Nexobrid group vs. $23.68 \%$ in the SOC group.

Conclusion: These preliminary datas need to be further investigated but the result seems to be important to merit further study and be preliminarily presented.

P055 Negative Pressure Wound Therapy (NPWT) in severely burned patients costs - use - risks

\section{B Wallner, M. Oehlbauer}

Traumacenter Murnau, MURNAU, Germany

Objectives: For 11 years NPWT has been transforming the management of severely burned patient in our Burn Unit. The conceptual and wide use of NPWT in extensive burns has become the golden standard. Costs, use and risks of this special treatment were reviewed in retrospect.

Methods: Our retrospective analysis included 590 patients with TBSA up to $85 \%$, who were treated with NPWT.

NPWT was used during every step of our burn treatment from the initial debridement up to the last step, the final defect coverage with split thickness skin graft after surgical debridement.

Material and personnel costs, as well as intensive care and surgical parameters of tissue repair were recorded during the entire treatment of burned patients. These parameters were compared to patients who received conservative treatment.

Risk assessment was developed for leaving the polyurethane foam on the wound longer than producers recommended.

Results: All patients with burn injuries treated with NPWT showed a significant reduction of redressing. The intensive care and surgical parameters of the patients treated with NPWT are more stable in comparison to conservatively treated patients. The catecholamine and analgesia need could be reduced due to a much lower risk of acquiring wound-infect associated sepsis.

Both the reduction of necessary dressing changes and the reduction of complications during the treatment with NPWT regarding wound sepsis and tissue repair, as well as the reduction of material and personnel costs, show that the application of NPWT in burn patients is a treatment which is highly cost-efficient.

Conclusion: The consistent and conceptual use of NPWT from the initial treatment to the final step of defect coverage shows, that after treating more than 500 burned patients, in particular severely burned patients, NPWT saves costs and offers numerous health benefits. NPWT is a treatment concept with an excellent rating in relation to costs, use and risks.

P056 Incidence and characteristics of non-accidental burns in children; a systematic review

MHJ Loos ${ }^{1}$, CAM Almekinders ${ }^{1}$, MW Heymans ${ }^{2}$, A De Vries ${ }^{3}$, R Bakx ${ }^{1}$

${ }^{1}$ Emma Children's Hospital, Amsterdam UMC, University of Amsterdam, AMSTERDAM, Nederland

${ }^{2}$ Amsterdam UMC, location VU University Medical Center, AMSTERDAM, Nederland 
${ }^{3}$ Rode Kruis Ziekenhuis, Burn Center Beverwijk, BEVERWIJK, Nederland

Objectives: The estimated incidence of non-accidental burns varies between $1-25 \%$ in children. Distinguishing non-accidental burns from accidental burns can be very complicated but is utmost importance for secondary prevention. The amount of studies concerning non-accidental burns are sufficient, however a clear overview is lacking.

Methods: The protocol of this systematic review was registered at PROSPERO. We searched literature in electronic databases published until July 2018, without language limitations. Two researchers screened, selected and graded the included articles, using standard methodology. We included primary studies of confirmed non-accidental burns in children. We excluded literature reviews, case-reports and unpublished data. We extracted data regarding demographics, burn characteristics, Child Protective Services (CPS) referral information and parent/household characteristics.

Results: 825 studies were screened, 17 were included. The incidence of non-accidental burns was pooled out of 10 studies and is $9,7 \%$. Indicators with a very high suspicion of intentional burns are deep partial thickness and full thickness burns, burns to the posterior trunk and burns caused by hot tap water. Indicators with a high to moderate suspicion of an intentional cause are burns to buttocks, genital and legs, a younger age of the child, additional injuries such as cutaneous injuries/bruises and fractures. More common caused by accidents are burns to head, neck, anterior trunk, upper extremities and feet. Little data is available regarding burns as a result of neglect. Quality of studies was often low to moderate mostly due to a high heterogeneity. This review is mainly based on retrospective studies.

Conclusion: The incidence of non-accidental burns in children is $9,7 \%$. Indicators with a very high suspicion of intentional burns are located at the posterior trunk, are deep partial thickness and full thickness burns and burns caused by hot tap water.

Picture 1: https://www.eventure-online.com/parthen-uploads/89/9EBA/add_1_540020_3fad07764c00-4868-8ccb-a48cf502c0d2.jpg

P057 A 3-year retrospective review of elderly patients with perineal and buttock burns and management at a regional burns unit in the United Kingdom

$\underline{\text { R Sinha }}{ }^{1}$, D Reissis ${ }^{1}$, N El-Muttardi ${ }^{1}$, Q Frew ${ }^{2}$, W. Khan ${ }^{1}$

${ }^{1} \mathrm{NHS}, \mathrm{BROOMFIELD}$, United Kingdom

${ }^{2}$ St Andrews Centre for Burns and Plastic Surgery, CHELMSFORD, United Kingdom

Objectives: Perineal and buttock burns are challenging wounds given risk of local contamination and difficulty in achieving stable dressings. These burns present unique hurdles in the elderly, given associated co-morbidities and frailty, which can negatively impact patient outcome. This study aimed to evaluate patient demographics, management and length of stay (LOS).

Methods: A retrospective review of patients over 65 years with perineal or buttock burns presenting to a regional burns unit over a 3 year period was performed. Patients with burns over $30 \%$ or who died from their injuries were excluded. Medical records were reviewed to assess patient age, sex, injury mechanism, total body surface area (TBSA), depth of burn, management approach and LOS.

Results: 40 patients presented over 3 years with 22 male patients and 18 female patients. The average patient age was 79 years. 32 patients had buttock burns with the majority (15 patients) sustaining a deep dermal burn. The majority (26 patients) had scald injuries. Buttock burns resulted in an average 3.77 day LOS per TBSA. 6 patients were managed surgically. 27 patients were managed conservatively.

8 patients sustained a perineal burn. 6 patients had a scald injury. 5 patients had superficial dermal thickness burns with average LOS per TBSA being 0.55 days. 1 patient was managed surgically, requiring an 11 day stay for a $7 \%$ flame burn resulting in 1.57 day LOS per TBSA. 7 patients were managed conservatively with a 0.15 day LOS per TBSA.

Conclusion: Scald burns are a prominent mechanism of burn injury. Buttock burns are associated with deep dermal burns and perineal burns with superficial dermal thickness burns. This informs pattern recognition of these burns in the elderly demographic. Buttock burns required a longer inpatient stay ( 3.77 days) compared to perineal burns ( 0.55 days). Perineal burns managed conservatively result 
in a shorter hospital stay.

P058 Efficacy and safety of decontamination with a hypertonic and amphoteric solution after chemical exposure

\section{A Navarro, L Mathieu}

PREVOR, VALMONDOIS, France

Objectives: Review the safety and efficacy of Diphoterine ${ }^{\circledR}$ solution, which is a hypertonic and amphoteric solution, for chemical burn treatment of skin and eyes.

Methods: Information sources were PubMed, Sciencedirect and Google Scholar. Terms included were Diphoterine, chemical burn irrigation, chemical injury, management, chemical burn emergency, Previn solution, skin decontamination and eye decontamination. Any study published through a peerreviewed journal was considered eligible. Published data must have included Diphoterine ${ }^{\circledR}$ solution or chemical lesions and decontamination management used. Various testimonials received from industries and organisations were added to this bibliographic research.

Results: The retrieved data could be classified as studies of safety or studies of efficacy.

Main safety results showed that Diphoterine ${ }^{\circledR}$ solution is non-irritant for skin and eyes, no cytotoxic and no toxic with acute oral and dermal LD50>200 $\mathrm{mg} / \mathrm{kg}$.

As to the efficacy of the solution, results could be divided in three categories.

First one is industry testimonies, more than 70 , concerning immediate use after a chemical splash.

Second one concerns chemical accident in industries, pre-hospital and hospital, more than 400 cases of irritant and corrosive.

Third one comprises a dozen comparative studies with more than 2700 cases washed with various solutions (water, saline or Diphoterine ${ }^{\circledR}$ ).

These documents helped to extract main conclusions about time of intervention, which we split in immediate intervention (industry) and delayed one (pre-hospital and hospital).

Conclusion: When hypertonic and amphoteric solution of Diphoterine ${ }^{\circledR}$ is used immediately after the accident, it often enables tissues to be preserved without any lesion. When used after a delayed wash, the need of medical treatment or surgery decreases and allows medical cares in best conditions.

In all cases, Diphoterine ${ }^{\circledR}$ solution helps to fix the chemical burning process.

P059 Retrospective analysis of tigecycline use in burn patients

Lipový, M Hanslianova, H Rihova, A Mertova, J Holoubek, F Raska, M Knoz, P Brychta

University Hospital Brno, BRNO, Czech Republic

Objectives: To evaluate our long-term experience with tigecycline use (alone or in combination with other antimicrobials) for treatment of skin and soft tissue infections (SSTIs) caused by several pathogens in patients with burns.

Methods: A monocentric, retrospective study enrolling all adult burn patients who were hospitalized in Department of Burns and Plastic Surgery University Hospital Brno in 2008-2018 and who received tigecycline therapy during hospitalization.

Results: A total of 98 patients with burns who received tigecycline therapy were identified in study period. The mean age of the patients in the group was $52.7 \pm 19.0$ years, male/female ratio was $2.5 / 1$, the average extent of burn area was $18.5 \pm 13.3 \%$ TBSA (total body surface area), the average length of hospital stay was $42.9 \pm 24.4$ days. A total of 54 patients (55.1\%) had inhalation injury confirmed with bronchoscopy. Average value of ABSI in patients was $7.2 \pm 1.8$.

Tigecycline was indicated for SSTIs therapy in all patients and in 59 patients $(60.2 \%)$ for multifocal infection. The average duration of use of tigecycline was $10.2 \pm 3.3$ days. In $58 \%$ of patients, tigecycline was administered as monotherapy and $42 \%$ in combination with other antimicrobial agents (amikacin, gentamicin, colistin, ciprofloxacin). Tigecycline was administered repeatedly during hospitalization in 8 patients $(8.2 \%)$.

Tigecycline was most commonly administered for infection caused by Klebsiella pneumoniae producing Extended Spectrum Beta Lactamase - ESBL (57 patients/58.2\%), Staphylococcus aureus methicillin sensitive (11 patients/11.2\%), coagulase negative Staphylococci (85 patients/86.7\%), 
Streptococcus pneumoniae (8 patients/8.2\%), Enterobacter cloacae (8 patients/8.2\%) and others.

Microorganisms were eradicated within tigecycline therapy in all patients, one of the patients in the group died (not associated with tigecycline therapy).

Conclusion: Tigecycline represents a safe therapeutic option for burn patients. There was no increasing resistance in pathogens isolated from our patients during the reporting period.

P060 Burn induced Erythema Treatment Using LDM (Local Dynamic Micro-Massage

\section{I.H. Lee}

Hangnagsoo hospital, SEOUL, South Korea

Objectives: For a long time, redness as a sequel to burn injury has been recognized as natural result and it is not a target of treatment. Recently, various therapeutic method (PDL; pulsed dye laser, antiredness cream) are introduced, but there is still no treatment of choice to post traumatic erythema. $\mathrm{LDM}^{\circledR}$ (Local Dynamic Micro-Massage, Wellcomet, Germany) uses two ultrasound waves of different frequencies with a rapid frequency modulation. Through this modulation new micro-massage effects can be obtained. We used an enhanced micro massage effect of LDM for improvement of post burn erythema.

Methods: We performed mainly once a week and twice a week in some cases. Outcome included Erythema grade for objective result, quartile clinical improvement score for symptom related subjective result.

Results: A total of 40 burn patients (mean age; 32.5 years, M:F=21:19) received 402 sessions (13.4sessions/ patient). Erythema grade fell from 4 to 1 by end of follow up period. Mean quartile clinical improvement score are 2.3.

Conclusion: Rapid oscillations of ultrasound frequencies produce a strong pulsation of the pressure gradients in the tissue, which gives rise to micro-massage. LDM $^{\circledR}$ has advantage of being painless and easy to apply and the effect is outstanding. It is thought to be able to use LDM as a single or a secondary treatment for post burn erythema.

P061 Extracorporeal shock wave therapy as adjunctive treatment for pathological post burn scars a prospective study an update

BP Dos Santos Marcano, J Aguilera Saez, J Serracanta Domenech, A Monte Soldado, JM Collado Delfa, G Antelo Coroas, E Verdaguer Olivella, JP Barret Nerín

Vall d'Hebron University Hospital, BARCELONA, Spain

Objectives: To identify if Extracorporeal shock wave therapy (ESWT) as an adjunctive treatment for patients with pathological post burn scar (PPBS) offers benefits over standard treatment alone

Methods: A prospective study was conducted in our center recruiting burn patients older than 18 years with PPBS greater than $1 \%$ TBSA located in a limb.

Patients were sequentially assigned using a non repetitive random series of numbers from one to forty where patients would be assorted as even or odd.

Both groups control and treatment received standard PPBS treatment, namely compression garments, silicone sheets, etc. The treatment group received ESWT two times a week for the first four weeks follow up.

Both groups were assessed on the day of recruitment day 0, at six weeks and six months.

Patients at all points were assessed using the Vancouver Scar Scale (VSS) and Visual Analogue Scales (VAS) for both pain and itchiness.

Results were analyzed using U Mann-Whitney and Wilcoxon

Results: Currently 29 patients have been recruited one abandoned the study.

So far median values for all three variables improved over time (table $1 \& 2$ ) and were better in the treatment group albeit not statistically so at every point (table 3).

Notably even at day 0 , the treatment group showed statistically significant more favorable values see (table 3).

Conclusion: Preliminary results indicates that patients on average showed improvement in all measured variables regardless of their assorted group, suggesting improvement over time and possibly 
the effects of the subjective nature of the variables.

Apparently, ESWT as an adjunctive treatment offers some improvement, but more precise measurements and in-depth analysis is required before considering its inclusion to the practitioner's armamentarium for pathologic scars post burn or otherwise.

Picture 1: https://www.eventure-online.com/parthen-uploads/89/9EBA/add_2_540917_94c44ee4efe1-4d52-98a5-88f2048a7ea9.png

Picture 2: https://www.eventure-online.com/parthen-uploads/89/9EBA/add_1_540917_94c44ee4efe1-4d52-98a5-88f2048a7ea9.png

Picture 3: https://www.eventure-online.com/parthen-uploads/89/9EBA/add_540917_94c44ee4efe1-4d52-98a5-88f2048a7ea9.png

P062 Using Intra-arterial tPA for Severe Frostbite Cases. An Observational Comparative Retrospective Study

MN Al Yafi', MA Danino², A Izadpanah², E Coeugniet $^{2}$

${ }^{1}$ McGill University, MONTREAL, Canada

${ }^{2}$ Universite de Montreal, MONTREAL, Canada

Objectives: Frostbite damages tissues through 5 major mechanisms out of which two are amenable to treatment. The first line treatment is rapid rewarming therapy using water at $40-42{ }^{\circ} \mathrm{C}$, that addresses the formation of ice crystals in the intra and extra cellular compartments. The second mechanism is progressive tissue ischemia after rewarming and is only accessible to a second line therapy represented by thrombolysis.

Methods: This study aimed to determine the efficacy of thrombolysis. This is a single center, retrospective cohort study, where it wasaimed to evaluate two groups of patients. A total of 18 patients participated in this study.

Results: Mean times between injury to thrombolytic therapy and admission to thrombolytic therapy, was 26.04 hours (SD 13.6) and 9.65 hours (SD 9.89), respectively. All patients suffered injuries ranging from 2 nd degree deep to 4 th degree. The rate of patients having complete, partial and no angiographic responses were $55.6 \%, 11.1 \%$ and $33.3 \%$ respectively. The main outcome of interest showed that $11(61.1 \%)$ patients in total had amputations at different levels. Results showed that in the intervention group, 5 (55.6\%) of the patients had amputations compared to $6(66.7 \%)$ from the control group $(\mathrm{p}=0.6)$ at comparable levels of amputation.

Conclusion: The literature supports that the use of IA-tPA might be beneficial for severe cases of frostbites, however it lacks of studies of major significance and results are often controversial. Our study has not shown statistically significant results on amputation levels and cannot support the hypothesis of efficacy of thrombolytic therapy.

\section{P063 INVESTIGATION OF THE FREQUENCY AND CAUSES OF RESPONSE TO CHILDREN PATIENTS WHO APPLIED TO BURN POLICLINIC}

\section{MSEYYAH, N ANAR}

KARTAL BURN CENTER, ISTANBUL, Turkey

Objectives: Despite medical and technological advances, burn is still a life-threatening serious problem. In this study, we wanted to evaluate the characteristics of 14 year-old and under-child patients who applied to Kartal Burn Center Policlinic during the winter months and who had contact burns.

METHOD: Kartal Burn Center between December 2018 and 2019 the months of February and contact the Polyclinic of pediatric patients under the age of 14 references burns, age, gender, area of burn, burn percentage, degree of burn were recorded.

Results: Of the 89 patients, $52(58.5 \%)$ were male and 37 (41.5\%) were female. The mean age was 2.3 years. The mean TBSA was 1.5 . $48 \%$ of the patients were in the 2 nd degree superficial, $40 \%$ in the 2 nd degree in the deep and $4 \%$ in the 3rd degree burn, and $8 \%$ in the 1 st degree burn. Burning agents 58 patients (65\%) stove contact, 14 iron contacts $(16 \%), 4$ pan contact $(4,5 \%), 3$ toaster $(3,5 \%), 3$ touches $(3,5 \%), 1$ the electric heater $(1 \%)$ was burned due to 1 furnace $(1 \%)$.

Conclusion: Burning is an important problem in pediatric patients. When the average age is 
considered low, the parents are careless in this regard, uneducated or even neglected is emerging. Especially in the winter heaters are used a lot. However, as with all heating sources, contact burns are common. However, the fact that all the patients we follow are preventable cases decreases the work of the parents when considering the age group. We think that the majority of these cases can be prevented with education and awareness.

\section{P064 PRESSURE COOKER BURNS}

\section{MSEYYAH, N ANAR}

KARTAL BURN CENTER, ISTANBUL, Turkey

Objectives: Burn can occur for many reasons. One of these is the pressure cookers that women often use to cook in the kitchen in a short time. Pressure cookers are pressure-controlled and when the pressure rises, it stimulates the person with a loud mechanism. Therefore, it saves time and is reliable in proper use. However, it may cause serious injury or burns if not handled properly. In this study, we have studied Kartal Burn Center Clinic for 3 months applied to the pressure of the pressure cooker as a result of the explosion of patients who wanted to evaluate the features.

Methods: Patients who applied to Kartal Burn Center Policlinic between 2018 December and 2019 February and burned due to a pressure cooker of explosion, age, sex, burn zone, burn percentage, burn degrees were recorded.

Results: $34(3,8 \%)$ male and 31 female (91,2\%) were female at the age of 34, with a mean age of 44 years. It was found that $35 \%$ of the patients were in the 2 nd degree superficial, $48 \%$ in the 2 nd degree in the deep and $8 \%$ in the 3rd degree burn, in the $9 \%$ in the 1st degree burn and in the total burn level the mean was $5.6 \%$. When we look at the burns, we observed that only 4 focused lower extremities were affected and the remaining 30 were affected by the chest, position, neck, arm, hand and wrist.

Conclusion: Burns are not affected by seasonal factors due to the pressure cooker and are more common in female population. The reason for this is that more women assume the task of cooking and in limited time we think that these burns are caused by the carelessness.

Picture 1: https://www.eventure-online.com/parthen-uploads/89/9EBA/add_1_541250_07324f4170f9-4ec3-b9b2-2c755e82ecc9.png

\section{P065 INVESTIGATION OF HOT WATER BAG BURNS APPLYING TO BURN POLICLINIC}

\section{MSEYYAH, N ANAR}

KARTAL BURN CENTER, ISTANBUL, Turkey

Objectives: People use hot water bags very often. Among the reasons for use, warming and reducing pain are the leading reasons. In our Study, Lütfi Kırdar Taining and Research Hospital Wound and Burns Center Outpatient Clinic in 3 months and hot water bag as a result of the explosion of the patients we wanted to evaluate the characteristics of burning.

Methods: The patients who were admitted to the Wound and Burn Center Policlinic who between 2018 December and 2019 February were recorded age, sex, burn zone, burn percentage, burn grade of the patients as a result of the explosion of hot water bag.

Results: 51 (67\%) of the 76 patients were female, $25(33 \%)$ were male, The mean age was 47.7 years. 29 patients were affected by foot, 8 patients were affected by back, 7 patients were affected by abdomen , 5 patients were affected by foot. The remaining patients were burned from the genital area, hand and leg areas. Total burn surface average was $4.90 .45 \%$ of the patients were in the 2nd degree superficial, $25 \%$ in the 2 nd degree deep and $8 \%$ in the $3 r$ degree, $22 \%$ in the 1 st degree burn, and the total burn surface average was $8 \%$.

Conclusion: the use of hot water bags is very common in our society. Knowing the cause of pain, using hot water bags can bring more harm than good. It is recommended to use with a sheath to prevent a possible explosion. In addition, quality and appropriate products should be used. Contact with skin may cause irritation. Direct contact may cause burns. Elderly and diabetic patients, especially those with additional disease, should use more caution.

Picture 1: https://www.eventure-online.com/parthen-uploads/89/9EBA/add_1_541592_ffe966fac558-4b9f-8b23-b32c3c643ddc.png 
P066 Economic analysis enzymatic debridement vs. standard of care in burn tretement preliminary data

\section{IMINIC, E Vigato, M Governa}

Azienda Ospedaliera Universitaria Integrata Verona, VERONA, Italy

Objectives: Cost analysis of the burn care is a well-defined challenge due to the large number and specialties of highly trained burn care personnel involved and extensive resources needed. The issue is getting more complex, due to different national, regional or even organizational reimbursement modalities. The aim of this study was to assess as much realistic possible cost of the burn care, issue by issue, in our Burn Center.

Methods: We analyzed retrospectively 44 patients divided in two equal groups; The SOC group and NXB group. The inclusion criteria for both Cohort group were the same. All the patients were with deep partial and full thickness burn. We compared different medical and economically important factors and measurements as cost-effectiveness parameters: analyzed days of hospitalization, timing to complete debridement, place of occupancy (BU or ICU or OT), human resources, devices and disposables consuming, blood consuming.

Results: The preliminary results has indicated an important decrees of important cost drivers in our reality, around $30 \%$ of savings, overall expenses in favor of NXB group. The confidential interval analysis confirmed the saving in all variables applied in our study.

Conclusion: The limit of this study is a small number of cases and its validity on Italian health care sistem. The real measure to weigh the merits of NXB MIM compared to SOC, will be the final outcome of function, cosmesis and quality of life two years post injury that should be at least as good as the SOC.

P067 Combination Therapy Using Nonablative Fractional Laser and Intralesional Triamcinolone Injection for Hypertrophic Scars and Keloids Treatment

\section{SHIN}

Yeouido St. Mary's Hospital, College of Medicine, The Catholic University of Kor, SEOUL, South Korea

Objectives: Combinations of various treatment modalities were proved to be more effective than monotherapy in treating hypertrophic scars and keloids including burn scars. This study was conducted to assess the effectiveness of combination therapy with nonablative fractional laser and intralesional steroid injection.

Methods: From May 2015 to June 2017, a total of 38 patients with hypertrophic scars or keloids were enrolled. 21 patients received steroid injection alone (control group) and 17 patients received 1550nm erbium-glass fractional laser and steroid injection simultaneously (combined group). The results were evaluated by analyzing the total number of treatment sessions, Patient and Observer Scar Assessment Scale (POSAS), recurrence rate and remission period.

Results: The mean number of treatment sessions was statistically fewer in the combined group than the control group ( 6.95 vs $5.47, \mathrm{p}=0.042$ ). There was a significant change in patient's scale as much as 14.62 in the control group and 22.82 in the combined group $(p=0.005)$, however, observer's scale score showed no significant difference (17.92 versus $20.55, \mathrm{p}=0.549)$. Total score showed the same tendency as in patient's scale (32.54 versus 43.36, $\mathrm{p}=0.041)$. The recurrence rate was $38.1 \%(8 / 21)$ and $35.3 \%(6 / 17)$, in the control and combined group respectively with no significant difference $(\mathrm{p}=0.859)$. However, the mean remission period was statistically longer in the combined group (3.00 months vs 4.17 months, $\mathrm{p}=0.042)$.

Conclusion: Combination therapy with nonablative fractional laser and intralesional steroid injection showed better results in the treatment of hypertrophic scars and keloids including burn scars with fewer number of treatment sessions, better patients' satisfactions, and longer remission periods.

Picture 1: https://www.eventure-online.com/parthen-uploads/89/9EBA/add_1_541946_897c454d5e9d-4944-82b7-aa3516b9be06.jpg

Caption 1: Hypertrophic scar in the foot dorsum developed after burn injury (left) and after 6 months from the last session of combination therapy (right). 
P068 Influence of drug-resistant epilepsy on surgical care of deep burn injury

\section{B.C. Certic}

Clinic for burns, plastic and reconstructive surgery, Clinical Center, Serbia, BELGRADE, Serbia

Objectives: Explaining the challenges in care and treatment of patients suffering from severe form of epilepsy.

Methods: Case repot of a 40 year old patient treated at the Clinic for Burns, Plastic, and Reconstructive surgery, Clinical Center of Serbia.

Results: We present the case of a 40 year old man patient with drug-resistant epilepsy who was admitted to our hospital with a deep 2 nd and 3 rd degree burn injury of 7\% TBSA. Multiple comorbidities presented on various organs and systems as he suffered from temporal lobe glioma, pancytopenia, erosive gastritis and eventually cachexia and decubital wounds. Hematological status was the most difficult issue to overcome as the patient had to be treated surgically and pancytopenia was thought to be associated with the patients every day anti-epilepsy drugs, which could not be excluded. The patient's mental state also had to be accounted for in treatment plans as the patient was usually not cooperative.

Conclusion: Accidental burn prevention should be advocated to severe epilepsy patients and especially their families, in an attempt to avoid burns in this group, as their care and treatment can be very challenging due to the fact of potential comorbidities which can hamper the success of surgical treatment.

\section{P069 RISK FACTORS FOR DELIRIUM AND RELATED OUTCOME IN ELDERLY PATIENTS WITH SEVERE BURNS; A RETROSPECTIVE COHORT STUDY}

\section{D.T. Van Yperen}

Burn Center Maasstad Hospital, ROTTERDAM, Netherlands

Objectives: The main objective of this study is to evaluate the prevalence of delirium in elderly burn centre patients and to identify risk factors contributing to the onset of delirium.

Methods: All patients aged 70 years and older admitted with burn injuries to Burn Centre Maasstad Hospital between 2011-2017 were eligible for inclusion. Data were retrospectively collected from patients' electronical medical files regarding patient and injury characteristics, the presence of delirium, potential risk factors for delirium and outcome after delirium.

Results: Ninety patients were included in this study. The prevalence for delirium was $13 \%(n=12)$. Risk factors for delirium were age (median 83.5 years (IQR 78.0 - 86.3) versus median 76.0 years $(72.0$ 83.3); p-value 0.04), ASA-score $\geq 3$ ( 8 patients (67\%) versus 23 patients ( $30 \%$ ); $\mathrm{p}$-value 0.02$)$, KATZ score $<6$ (9 patients (82\%) versus 30 patients $(40 \%)$; p-value 0.01$)$ and the use of anticholinergic drugs $(9$ patients $(75 \%)$ versus 22 patients $(28 \%)$; p-value $<0.01)$. Delirium resulted in prolonged hospital stay (median 26.5 days (IQR 16.8 - 54.3) versus median 19.0 days (IQR 9.0 - 26.3); p-value 0.02 ) and intensive care unit (ICU) stay (median 5.5 days (IQR 2.75 - 9.75) versus median 1.0 days (IQR 1.0 - 2.5); p-value $0.01)$. Mortality was increased after six months ( $42 \%$ versus $8 \%$; p-value $<0.01)$ and after 12 months ( $42 \%$ versus $9 \%$; $p$-value $<0.01$ ).

Conclusion: Delirium is frequently diagnosed in elderly burn centre patients and is related to poor outcome including prolonged hospital and ICU stay and increased mortality. Increased age, poor physical health status, physical impairment and the use of anticholinergic drugs may contribute to the onset of delirium. To investigate whether the prevalence of delirium in elderly burn centre patients can be reduced we will perform a multifaceted intervention study.

P070 Gender differences in self-inflicted burns

$\underline{\text { SG Fidel-Kinori1 }}{ }^{1}$ C Castellano-Tejedor ${ }^{2}$, MS Cepeda-Diez ${ }^{1}$, JP Barret-Nerin ${ }^{1}$

${ }^{1}$ Hospital Universitario Vall d'Hebron, BARCELONA, Spain

${ }^{2}$ RE.FIT Research group Parc Sanitari Pere Virgili, BARCELONA, Spain

Objectives: to analyze if gender-related patterns of self-inflicted burns exist to describe prototypical profiles between males and females 
Methods: This cross-sectional study is based on burn case-reports $(\mathrm{N}=51)$ from an acute specialized burns unit (SBU) from a reference hospital in Catalonia, Spain (2010-2018). Data regarding age, sex, triggers for self-inflicted burns, total burn surface area (TBSA), methods and circumstances of the injury, and other relevant clinical variables were collected.

Results: Mean age of the whole sample was 42,16+-13,24 and most of them were males $(n=38,74$, $5 \%)$, from Spain $(n=37,72,5 \%) .66 \%(n=31)$, were single, finished elementary education $(n=38,84,4 \%)$ and were unemployed in $64,5 \%$. In $68,8 \%$ cases the self-inflicted burn was clearly an autolytic attempt $(n=35)$. Main triggers were psychopathology $(48,8 \%)$ and familial conflicts $(34,1 \%)$. The most prevalent psychopathological diagnosis were bipolar disorder, major depression and schizophrenia( $25 \%, 22,9 \%$ and $20,8 \%$ respectively).

Main method was to set fire on own bodies/clothes ( $n=42,82,4 \%$ ), and the number of previous attempts range between 0 and 6 (mean 1,67+-1,57).

Differences between genders were observed for the intention of the self-inflicted burns with significantly more males presenting these injuries as a clear autolytic attempt $\left(X^{2}=4,093, p=0.043\right)$

Conclusion: Most patients with self-inflicted burns in our study were men, with clear autolytic intentions. Considering the high prevalence of psychiatric disorders among the sample, both gender included, it cannot be concluded that they present higher risk factors compare to females or use more aggressive methods.

P071 Recovery to pre-injury HRQL during 18 months following burns: The effects of burn severity and PTSS

E Boersma $^{1}$, H Hofland ${ }^{2}$, A De Jong ${ }^{3}$, A Boekelaar ${ }^{3}$, E Vandermeulen4, A Goemanne ${ }^{5}$, J Meijer ${ }^{6}$, N Van Loey ${ }^{1}$

${ }^{1} \mathrm{ADBC}, \mathrm{BEVERWIJK}$, Netherlands

${ }^{2}$ Maasstad Hospital, ROTTERDAM, Nederland

${ }^{3}$ Red Cross Hospital, BEVERWIJK, Nederland

${ }^{4}$ Military Hospital, BRUSSELS, Belgium

${ }^{5}$ University Hospital, GHENT, Belgium

${ }^{6}$ Martini Hospital, GRONINGEN, Nederland

Objectives: Burn injuries can impact health-related quality of life (HRQL). We investigated extent of HRQL recovery to pre-burn level from hospitalization up to 18 months post-burn in relation to burn severity and post-traumatic stress symptoms (PTSS). A second objective was to investigate the suitability of the EQ-5D for retrospective proxy report.

Methods: The sample consisted of 480 adult burn patients admitted to burn centres in the Netherlands or Belgium. The EQ-5D + cognitive dimension was used to evaluate HRQL shortly after admission to a burn centre and after 3,6,12 and 18 months follow-up. Also, retrospective pre-injury EQ$5 \mathrm{D}$ scores were collected from patients and partners $(\mathrm{N}=119)$. Burn severity (number of surgeries) and six months post-burn PTSS were collected as predictors of HRQL.

Results: Over time more patients returned to pre-injury HRQL status in each dimension, except Cognition. Burn severity showed a significant predictor of not returning to pre-injury levels of the EQ5D dimensions mobility, self-care, usual activities, pain, cognition and the summary and VAS score over time. PTSS significantly also predicted not returning to pre-injury levels in these dimensions over time, but to a lesser extent. For the Anxiety/Depression dimension, only PTSS was a significant predictor, not burn severity. The retrospective pre-injury measure showed substantial agreement between patient and partners.

Conclusion: In general, patients who had more severe burns or higher levels of PTSS were more at risk of not returning to pre-injury HRQL over time. The substantial levels of patient-partner agreement on pre-injury HRQL suggest the EQ-5D can be used as a retrospective measurements by proxy.

P072 Digital opportunities in the burn patients rehabilitation

\section{IVuonoranta}


HUS Helsinki University Hospital, ESPOO, Finland

Objectives: The treatment of burn injury patients has been centralized the Burn Center of Finland, which means that the treatment of many patients is located far from their hometown. In the hometowns of the patients where continuous rehabilitation is given, physiotherapists and occupational therapists often have little experience of burn injuries. Digital services could be utilized in the future rehabilitation of the patients. The purpose of this study was to find out how digital services could be utilized in the continuing rehabilitation of burn patients, from a staff perspective. The idea was also to research what kind of instructions, guidance and support therapists find to be useful.

Methods: Data was collected using internet based questionnaire. The participants of the study were physiotherapists and occupational therapists who during the years 2016-2017 have been giving rehabilitation to a burn patient, after being treated at the Burn Center of Finland. In total, the survey was sent to 41 therapists, of which 15 answered.

Results: The results of the study showed that digital services could be useful during different stages of the rehabilitation process of burn patients. Fluent communication between the various healthcare institutions is the key to successful rehabilitation. Respondents felt that digital services would be useful in the future. Some themes could clearly be highlighted from the answers. The issues that came up from the responses were instructions for professionals, real-time remote meetings, and personalized digital services for the patients.

Conclusion: The demand of digital services for burn patients is clear, and the requirement is high for this patient group. According to the results of the study, there is also interest and motivation among the staff. The results of this study can be utilized when developing digital services for burn patients in Rehabilitation hub. Rehabilitation hub is a part of the Virtual Hospital 2.0 project.

P073 Lipofilling influence on the tissue structure of the skin scars

P Badiul ${ }^{1}$, S Sliesarenko' ${ }^{1}$, I Baranov ${ }^{1}$, N Nor ${ }^{2}$, K Tsyhankov ${ }^{3}$

${ }^{1}$ City Hospital \#2, DNIPRO, Ukraine

${ }^{2} \mathrm{SE}$ „Dnipropetrovsk Medical Academy of Health Ministry of Ukraine,,, DNIPRO, Ukraine

${ }^{3} \mathrm{SE}$ „Dnipropetrovsk City Multi-profile Clinical Hospital \# 4 of DCC, DNIPRO, Ukraine

Objectives: Morphological grounding of applying the lipofilling operation for healing skin scars.

Methods: 25 patients with skin scars after deep burns, who were undergoing treatment from 2014 till 2018, took part in the research. The histological examination was carried out using biopsy from areas in the scar tissue. The sampling materials took place before the operation and on the $14^{\text {th }}$ day after the lipofilling operation.

Results: The histological picture of the patients with atrophic and hypertrophic scars before the operation was characterized by similar pathological changes in the form of atrophic changes in epidermis with excessive pigmentation of the cells in the basal layer, skin appendages were sharply atrophic or absent. In the derma, the roughening of pulls of collagen fibers, hyalinosis of collagen fibers were observed; it was mainly manifested in the middle and lower thirds of the reticular layer, where band-shaped, ribbon-like areas of hyalinosis were formed. In deep areas of derma, uneven intermittent edemas were revealed with the basophilia of connective tissue elements, a large number of variegated vessels. On the $14^{\text {th }}$ day after the operation the epidermis had a regular structure, the papillary and reticular layers of derma were represented by the unformed connective tissue with the significantly expressed capillary network and the insignificant center of perivascular lymphohistiocytic infiltration. The collagen fibers were well-contoured, formed the network of multidirectional pulls with clearly determined slit-like gaps between them. Occasionally small round cavities, which contained fat protein detritus, were revealed. Singular small areas of maturing granular tissues with mono- and multi-vesicular structures were determined.

Conclusion: The speed and completion of the regeneration processes in skin depends on the condition of the hypodermis adipose tissue. Lipofilling could be considered as a method for the substitutive therapy at morpho-functional insufficiency of the hypoderm. 


\section{$\underline{\text { RT Tasanen }}$}

Helsinki Burn Centre, ESPOO, Finland

Objectives: Healing physically from a burn injury is only one step in the process of rehabilitation. After hospitalization burn survivors have to face challenges such as scarring and altered appearance. Many survivors find peer support to be one of the most helpful resources in the recovery process. Little research is available on the role of peer support in post burn recovery.

This presentation describes the implementation of peer support for burn survivors in sparsely populated country with long distances and a few actors. Main focus is to increase the awareness of healthcare professionals about the importance of peer support in this patient group.

Methods: Co-operation with two active players of Finnish burn survivors' organizations.

Results: National Burn Survivor (NBS) organization of Finland organizes every summer and autumn a family camp meeting for burn survivors and their families. Aim of these camps is get together and meet peers who have been through the process of burn recovery. Maintaining the network of supporters and cooperation is of uttermost importance.

NBS is a member of Finnish Allergy, Skin and Asthma Federation, which trains supporters, maintains closed Facebook group and offers web-based rehabilitation.

Association of Children Injured Accidentally organize family camps and adaptation training for burnt children and teens. Websites for families include information on burns and support available.

Conclusion: More research should be obtained from burn survivors' views of peer support. How do they view this intervention and services of NBS?

To ensure this valuable voluntary activity, more trained supporters, especially young people, are needed. Web-based rehabilitation is a trend of the day, but does it replace face-to-face support?

More attention should be paid to the knowledge of health care professionals about this resource and its potential in the recovery process of burns.

P076 A case report focused on pressure treatment and splint options for a 62\%TBSA flame burn patient

\section{A E Piironen, S P Lempiäinen}

Helsinki University Hospital, ESPOO, Finland

Objectives: The objective of this case report is to show the scar treatment products used on an adult patient with $62 \%$ TBSA third and second-degree flame burns. 50-55\% of the body surface was covered by split thickness skin grafts. Different kinds of static and dynamic splints were used from the acute phase through the rehabilitation process to prevent loss of ROM and functional problems especially in the upper body and extremities. Majority of grafted skin and donor sites developed hypertrophic scarring that needed treatment with various silicone products and pressure garments. Active physiotherapy was also given throughout the rehabilitation process. Duration of time spent combined in the ICU and hospital ward was 4 months and 4 days. Active scar treatments and regular visits to the occupational therapist were continued after discharge for three years. During this time also three scar repair surgeries were performed.

Methods: Data was gathered from patients' medical reports and pictures taken throughout the wound healing and scar treatment process. The effect of scar treatments and the progress of the scar development process were evaluated subjectively by the patient and objectively by the occupational therapist. Evaluation methods used were interviewing, discussion, observation and palpation.

Results: After three years from the burn injury patient has mild tightness on her upper body during rotation of head and walking. Functionally patient has recovered well and is able to work and do leisure activities such as dancing and skiing.

Conclusion: Patient needed a few scar repair surgeries despite active scar treatment and physiotherapy. Scar treatment process was overall successful, and patient showed clear relief from scar treatments for symptoms such as itching, pain, tingling, tightness and inflexibility of the scar.

P077 Application of kinesiotape on syndactyly in deep burned hands. Pilot study.

L Grossi Garriga, E Santacreu Santacreu, S López Lebrato, ML Torrent Bertran 
Hospital Universitari Vall d'Hebron, BARCELONA, Spain

Objectives: Deep burns on hands may cause sequels, one of them could be the syndactyly, which is difficult to treat and usually need reconstructive surgery.

Kinesiotape is a cotton elastic band with huge capacity for traction, elasticity and adaptability, respecting the fragility of burned skin. Use of kinesiotape enables to exert pressure and tension to the interdigital skin, hard to achieve with pressotherapy gloves. Between the syndactyly and kinesiotape, we put a soft material to protect the skin from the stretching and allow us to create a pseudo-occlusion environment.

Our main objective is proving than kinesiotape bands are useful tools for controlling and /or decreasing syndactyly appearance.

Methods: We present a pilot study with four cases with deep burns affecting interdigital spaces, in which we early applied pressure with kinesiotape, until the scar maturation.

Kinesiotape was placed into interdigital spaces, weared 24 hours and changed daily.

A weekly monitoring was made, measuring with goniometer the opening of interdigital spaces, DASH for hand functionality, Vancouver scale for scar evolution and Likert Scale for patient satisfaction.

Results: Satisfactory results were obtained in all parameters studied at discharge.

Conclusion: As a conclusion, seems that use of kinesiotape can be a useful tool to prevent syndactyly appearance.

P078 Effectiveness of self-adherent wraps for edema and hypertrophic scars in burned hands. A pilot study

L Grossi Garriga, E Santacreu Santacreu, S López Lebrato, ML Torrent Bertran

Hospital Universitari Vall d'Hebron, BARCELONA, Spain

Objectives: Persisting edema and emergence of hypertrophic scars in burned hands cause a huge impact on functionality and quality of life's patients.

Some authors have revealed that using a mechanical pressure can improve both complications.

Unlike pressotherapy garments, in which a wound healing is needed to put it on, self-adherents wraps allows us to begin the treatment in an early stage.

The use of self-adherent wraps in burned hands in an acute and post-surgery phases allows to improve edema and functionality, and an early control of hypertrophic/ shrink scar appearance.

Methods: A pilot study was made with 5 patients with hands burned 2nd and 3rd degree. All patients presented edema and wounds, not allowing putting in pressotherapy gloves.

The beginning of self-adherent bandage was realized in 4 patients in acute phase (2-3 weeks post surgery). The other patient begin the bandages later, in a subacute phase. An early self-adhesive bandage was placed, retired for the treatment and re-placed daily after rehabilitation treatment.

Weekly evaluations were made until the pressotherapy glove was given: tape measure for edema, goniometer for the movement, DASH questionnaire for functionality and Vancouver scale for the scar evolution

Results: In all patients we observed a substantial decrease of edema, improvement of functionality and scar aspect.

Conclusion: The use of self-adherent wraps in early phases of the treatment of the burns encourages the decrease of edema and improves functionality, being a coadjutant treatment to base treatment.

P079 Between postural control and psychomotor development. A clinical case

L Grossi Garriga, E Santacreu Santacreu, S López Lebrato, ML Torrent Bertran

Hospital Universitari Vall d'Hebron, BARCELONA, Spain

Objectives: M., suffered scald burns in $42 \%$ of the body at the age of 13 months, located in both lower extremities, lower back, infer-umbilical area, right flank, left hand and forearm. Previously to the injury, the child was in crawling phase, standing up occasionally and beginning lateral walking.

He required three surgeries, and despite the early beginning of physiotherapy and occupational 
therapy while hospitalized, he presented hypertrophic scars and contractures, especially important in the back of the legs and forward of the ankles.

Methods: Daily rehabilitation treatment during this period combined drainage and bandage techniques for decrease edema and open syndactylies, prolonged skin stretching and treatment for the psychomotor development.

Results: At discharge due to family moving to another country, M., then 16 months old, was able to crawl, to stand up by himself and walk laterally, and was beginning forward walking with assistance.

Conclusion: In this case, we want to show the dilemma we sometimes have, as therapists, between the conventional treatment for burns, that would encourage a continuous knee extension to avoid skin retraction, and the treatment for some specific cases, like psychomotor development for babies and child, focused in crawling as means of locomotion, exploration and preparation of the abilities needed for walking.

\section{P080 RESPIRATORY REHABILITATION PROTOCOL FOR PATIENTS WITH COMPLEX BURN INJURIES}

ML Torrent-Bertran, D Issa-Benitez, S López-Lebrato, E Santacreu- Santacreu, B Planas-Pascual, L Grossi-Garriga, J Serracanta, A Gómez-Garrido

Vall d'Hebron University Hospital, BARCELONA, Spain

Objectives: Patients with complex burn injuries require interdisciplinary rehabilitation to maximize their functional recovery.

Burn-injury patients have often been exposed to smoke inhalation and treated with intubation/ tracheostomy for long periods. In addition, the large burns may have affected the thorax, which implies a difficulty in thoracic expansion. It is important to study the respiratory function and to be able to offer a respiratory physiotherapy program.

The management of the burned patient is done in specialized centers, with specific rehabilitation protocols designed to work on different areas, including the respiratory system.

The aim of this study is to create a respiratory rehabilitation protocol for the burned patient.

Methods: A bibliographic search was carried out on respiratory physiotherapy and on respiratory complications after a burn.

A group of experts from the interdisciplinary rehabilitation team was formed, consisting of a physiatrist specialized in burnings, others in pulmonary rehabilitation, a burn physiotherapist and a respiratory physiotherapist and prepared the protocol of action.

Finally, this protocol was presented to the multidisciplinary team and starts to be used systematically

Results: In the acute phase, the patients are evaluated by the rehabilitation physician of the burn unit. The usual protocol begins with exercises of respiratory physiotherapy.

The subsequent assessment consists in studying the respiratory function, the respiratory muscle strength and the functional capacity.

According to the results obtained, the rehabilitation program is adapted, including respiratory muscle training, endurance and resistance training.

Before the patient leaves the hospital, he is reevaluated to assess the necessity to follow outpatient treatment.

Conclusion: Patients with complex burns require interdisciplinary rehabilitation throughout their care. It is important to study the respiratory functionality, and thus be able to carry out a respiratory rehabilitation program with the aim of improving the functionality and respiratory function of these patients at discharge.

P081 NURSING CARE AND PHYSIOTHERAPY IN THE BURN INTENSIVE CARE UNIT OF THE INDIVIDUAL WITH TOXIC EPIDERMAL NECROLYSIS

\section{MSEYYAH, Ö YASAN}

KARTAL BURN CENTER, ISTANBUL, Turkey

Objectives: OBJECTIVE: Stevens-Johnson syndrome (SJS) and Toxic epidermal necrolysis (TEN) 
are severe adverse cutaneous drug reaction characterized by epidermal loss and multisite mucositis. The aim of nursing care of the patient and physiotherapy process with SJS is to enhance the patient's quality of life.

Methods: CASE: 17-year old male patient, no additional history of illness. About 6 weeks of vitamin $B$ treatment and paracetamol applied. When he applied to the emergency room, there were lesions in the oral mucosa and eyes. Tracheal intubation was performed. Body, both upper extremities, Head Primary and common skin rashes were present. The patient's age, allergy condition, chronic illness, etc. Patient was given oral care, perineum care, body cleaning, bed care, every 2 hours. A special silver product was used to reduce lesions and sepsis in the upper body of the patient and changed over the course of the day.

Patient hospitalization 6. extubation was attempted on his day, but because he was unable to tolerate, he was intubated again. Pain, agitation, oral nutrition were closely monitored. It was supported as TPN and enteral for nutrition.

The patient who was extubated again remained intubated for 528 hours. After 35 days in intensive care, the burn service was transferred to the burn service. He was discharged after 16 days in burn service.

Results: The range of motion of active joints was assessed by goniometric, pain by VAS, muscle strength by manual muscle test, daily life activities and transfer activities by Barthel Index. The physiotherapy program included active respiration techniques, forced expiration technique, respiratory control, diaphragmatic respiratory exercises, in-bed exercises and activities for functional purposes. After rehabilitation, there was an increase in active range of motion (EHA) and functional activity, and a decrease in pain.

Conclusion: We think that early physiotherapy practices will reduce the negative effects of immobilization.

Picture 1: https://www.eventure-online.com/parthen-uploads/89/9EBA/add_1_541538_94f83db2a4cd-4b05-8d71-63bfe1e68fa8.png

\section{P082 A DIFFERENT COMPLICATION IN ELECTRICAL BURNS}

\section{MSEYYAH, Ö YASAN}

KARTAL BURN CENTER, ISTANBUL, Turkey

Objectives: OBJECTIVE: High voltage electric shocks $(1000 \mathrm{~V}, 50 \mathrm{~Hz})$ are usually seen in electricians. Electrical burns have many complications. In our case, the region exposed to high tension was the front of the trachea and the trunk.

Methods: CASE: 41 years old, male, with no additional disease, $1 \% 4$ th degree on the front face of the neck as a result of exposure to electric, body front face, right and left upper arm, right forearm, right foot 2nd degree (TBSA8\%). When the patient arrived, he had intubated, sedated and inotropic support.

Plastic surgery applied debridement to the necrotic area of the patient's neck. The defect area measurements were taken and the appropriate local transyFlebi was planned. Then the flap was removed and sutured with the appropriate sutures. When we fed the patient, he was on the run from that area, and he was at risk of infection. Debridement was performed by surgeons in other burn areas and closed with burn products. Right foot 5 . The finger was amputated on the 15th day of admission. The patient had an arterial hemorrhage in the neck area. Tracheostomy was performed by entubation for 26 days. Patients were intubated for 816 hours during the intensive care unit. Then, a special catheter was operated by the ear nose throat team. As a result, the patient was provided with a comfortable meal.

In the nursing care process, Bleeding Control, Oral Care, tracheostomy care, perine care, Dren follow-up, nasogastric, aspiration, nutrition, pain, 2 hour position, catheter care was routinely applied.

Results: Since the patient was accepted in the physiotherapy program, edema control, positioning, splinting, passive and active exercises for ROM and respiratory physiotherapy were started.

Conclusion: Electrical burns are very common in our country and treatment is a trauma that requires a multidisciplinary approach.

Picture 1: https://www.eventure-online.com/parthen-uploads/89/9EBA/add_1_541559_71499fa8- 
a00c-4d8d-a577-0d8752aabf48.png

P083 Alternating Two-Piece Facial Orthosis: A novel design for improved outcomes

T] Boulter

Children's Hospital Colorado, AURORA, USA

Objectives: The use of a facial orthosis is a critical component to improve outcomes following a burn injury to the face. Clinicians must consider mobility, tissue length and dynamic muscle control while adhering to the scar management principles of: pressure therapy, use of silicone gel products, massage, facial exercises and use of an orthosis. We hypothesize that utilizing an alternating two-piece facial orthosis will improve outcomes versus a traditional full-face mask.

Methods: A full facial orthosis provides pressure to all involved areas while maximizing tissue length. This results in the orthosis covering the entire face.

This two-piece orthotic design, maximizes tissue length by stabilizing each portion of the orthosis with bony landmarks of the face. With stability of the upper portion of the face, tissue is gently mobilized with movements of the mouth. The lower portion of this design stabilizes the chin and lower portion of the face, allowing gentle mobilization with facial expressions.

Results: The two-part facial orthosis decreases the need for frequent donning and doffing for meals and exercise. The design allows the child to easily transition to full time wear alternating between the upper and lower portions. This design also allows for increased ease and specificity for modifications. Further, the Two-piece orthosis decreases the impact on the growing skeleton, optimizes tissue length at highly mobile areas of the face and increases skin tolerance due to changes in the forces of the orthosis and the associated strapping.

Conclusion: The deforming forces of a facial burn impacts the whole child; the growing skeleton, psychosocial reintegration, and functional skills including eating, vision and phonation. The use of a two-piece facial orthosis allows optimal scar management, functional independence and improved facial mobility.

Picture 1: https://www.eventure-online.com/parthen-uploads/89/9EBA/add_1_541886_c1abe390aa04-4401-8f0b-aeea33be9f6e.jpg

Caption 1: examples of two piece facial orthosis

\section{P084 PHYSIOTHERAPY IN PATIENTS WITH BURNING BASED ON EVIDENCE.}

\section{G VELOSO NUNES}

UniRV, VILLENEUVE LE ROI, France

Objectives: the study aimed to evidence the evidence of physiotherapeutic rehabilitation in the burned patien

Methods: a review of the literature on burned physiotherapy using electronic database Lilacs, Scielo, Pubmed, with inclusion criteria of works that had a practical intervention of the physiotherapy in the burned, being analyzed and demonstrated by means of table to number of participants and results of each study.

Results: Five studies were included according to the inclusion and exclusion criteria, where the conducts and results of each study were evidenced. The studies analyzed had positive impacts on the results of physical therapy in patients with burns

Conclusion: physiotherapy is of paramount importance for the treatment of the burned patient aiming at a long-term approach, where the physiotherapeutic care of the burn victim should begin on the day of the injury.

Picture 1: https://www.eventure-online.com/parthen-uploads/89/9EBA/add_1_546140_05fd9e18a8d4-4c15-ada1-27c4f3a8b845.png

\section{P085 DELAYED SURGICAL NECRECTOMY OF BURN WOUND AFTER INITIAL SPECIAL DRESSING TREATMENT}

A. Stritar, L. Emeršic, S. Stevovska, A. Arhar 
University Medical Centre Ljubljana, LJUBLJANA, Slovenia

Objectives: It sometimes proves difficult to define burn depth when treating mid-dermal burns, flash burns, scalds or chemical lesions. In these undefined cases it is possible to initially introduce special burn dressings with their own mechanical and pharmacodynamic characteristics. Wound dynamic is monitored when changing the dressings. Should wound healing stop, surgical procedure is required.

Methods: During the first three days burns of unspecified degree are treated using wet dressings, afterwards special dressings are applied. Wound dressing diminishes inflammation, infection and prevents deepening of the wound. It is in this manner that vital dermis and wound healing potential are preserved. Apart from healing by first intention, the granulation phase is prolonged in some areas of the wound preventing the process of epithelisation, which results in delayed wound healing. In those cases surgical therapy of slough as secondary necrectomy is indicated.

Results: Five patients that were initially treated with special dressings only were analysed. Surgical treatment of some of the unhealed areas was performed. The population comprises three children (10$15 \%$ TBSA) and two elderly patients (15-20\%TBSA).

Conclusion: The case in point is an individual, selective, yet active approach. Regarding middermal burns, demarcation of the burn wound is expected that would not gradually heal in its entirety were conservative therapy only to be applied. Indeed, delayed surgical necrectomy decreases the size of the surgical area and skin graft needed. This is especially important in certain populations, i.e. children and elderly patients. A thoughtful surgical management results in an optimal ratio between an operative and conservative approach of burn wound treatment.

Finally, multidisciplinary care is required, which includes microbiological diagnostics, targeted antibiotic therapy, glycaemic regulation, nutritive support and physical therapy up until complete rehabilitation.

\section{P086 THE USING OF WOUND COVERINGS IN SURGICAL TREATMENT OF BURNS IN REGIONAL BURN CENTRE}

\section{S.B. Bogdanov, A.V. Poliakov}

Scientific Research Institute - Ochapovsky Regional Clinic Hospital ?1, KRASNODAR, Russia

Objectives: The aim of our study was to analyze the results of treatment of burn patients using "Suprathel" in Krasnodar burn department of "Scientific Research Institute - Ochapovsky Regional Clinic Hospital No. 1".

Methods: We use early surgical treatment of burnt patients during about 25 years. Patients with deep dermal burns were performed necrectomy in 2-5 days after injury. After necrectomy we used skin graft plasty and wound dressing. In order to create optimal conditions for the epithelialization of deep dermal burns after necrectomy we applied "Suprathel" since 2015. After necrectomy to the lower layers of the dermis we used napkins with epinephrine hemostasis for 5-7 minutes. Then superimposed sterile wound covering "Suprathel", which is fixed with a bandage.

Results: Subsequently, only cosmetic dressings performed starting from the third day after surgery. Wound coatings were not removed until complete epithelialization of wounds. Epithelialization occurs depending on the depth of burns 8-12 days after surgery. When staged surgical treatment of painful dressings with ointments, without "Suprathel", made every other day for 15-21 days.

Conclusion: In the traditional surgical treatment of deep dermal burns tend to deepen with the formation of granulation tissue and the need to follow skin graft plasty. A more preferred method of treatment of deep burns is a method of early surgical treatment, in which the skin necrosis is removed in the early stages after the burn, and the wound is closed wound coverings. We got faster wound epithelization with the use of "Suprathel" with large areas of burns. Research result has been an advantage once the surgical treatment of wounds using "Suprathel" to the local conservative treatment with long-lasting dressings.

P087 The role of nanocellulose dressing (Epicite $\mathbb{R})$ in compartment syndrome. 


\section{IB Ruiz-Padilla}

Hospital H+, SAN MIGUEL DE ALLENDE, Mexico

Objectives: Show the experience with combined release surgery therapy, Epicite ${ }^{\circledR}$, and V.A.C. compared with those who underwent surgery and coverage with silver sulfadiazine in compartmen syndrome.

Methods: A prospective study was conducted with two groups, number 1 consists of surgical release covered with silver sulfadiazine (traditional); number 2 is the surgical release covered with Epicite ${ }^{\circledR}$ and V.A.C.

Results: We included 5 adult patients and 10 extremities (bilateral upper compartment syndrome). In both groups, normal pressure values were obtained after release. The first wounds group, sulfadiazine cover, had more eschar formation, areas of maceration and more difficulty for fasciotomy closure. Those of the second group presented better granulation tissue, scarce macerated tissue, eschar that come off more easily, as well as a simpler closure.

Conclusion: The compartment syndrome is an entity that occurs in patients with electrical burns, fire, etc., which involve an increase in intra-compartment pressure, particularly in the arms and legs. The standard treatment is surgical release to decrease that pressure, as soon as the diagnosis is made. On a regular basis the wounds are covered with silver sulfadiazine in the form of cream. Because the results in relation to the wound are not optimal, we sought the use of a dressing that combined resistance, absorption, tolerance, handling, finding that nanocellulose microfibers (Epicite ${ }^{\circledR}$ ), give us a superior results.

P088 Our experience of preparation patient with neck burn to tracheostomy

D.O. Vagner ${ }^{1}$, E.V. Zinoviev ${ }^{1}$, I.V. Shlyk ${ }^{2}$

${ }^{1}$ St. Petersburg Institute of Emergency Care named after I.I. Dzhanelidze, SAINT-PETERSBURG, Russia

${ }^{2}$ Pavlov First St. Petersburg State Medical University, SAINT-PETERSBURG, Russia

Objectives: The main indications for tracheal intubation and respiratory support in patients with burns are inhalation injury, extensive burns and limited area burns of the face and neck. The duration of tracheal intubation in these patients may be as long as 20-30 days or more, which significantly increases the risk of developing post-intubation complications. The imposition of a tracheostomy can reduce the incidence of life-threatening complications. However, an objective obstacle to the implementation of this intervention is burn of the front surface of the neck. The solution is early fascial excision of the area of a deep burn with simultaneous skin grafting on the front surface of the neck and chest.

Methods: The retrospective study included 24 patients treated in the burn ICU. All patients underwent early necrectomy with simultaneous autodermoplasty on the front surface of the neck. The average age of the patients was $43.4 \pm 17.1$ years, the burn area was from $20 \%$ to $87 \%$ of TBSA.

Results: The above operation was performed on the $1^{\text {st }}$ day of the injury in 2 patients, on the $2^{\text {nd }}$ day - in 4 , on the $3^{\text {rd }}$ day - in 7 , on the $4^{\text {th }}$ day - in 11 patients. Complete engraftment was found in 18 patients, partial engraftment in 6 cases. Subsequently, a tracheostomy was performed in 21 of 24 (88\%) patients through the restored skin in $10.6 \pm 1.5$ days after injury.

Conclusion: Early fascial excision with simultaneous skin grafting on the front surface of the neck makes it possible to safely perform a tracheostomy 9-11 days after the burn. This operation is a technically simple and safe method for treating patients with severe burns.

Picture 1: https://www.eventure-online.com/parthen-uploads/89/9EBA/add_539810_dd4724fd6e74-4e38-8832-81d29cd50f41. 2 Skin graft in 2-nd day.jpg

Caption 1: skin graft simultaneous with necrectomy in 2-nd day

Picture 2: https://www.eventure-online.com/parthen-uploads/89/9EBA/add_1_539810_dd4724fd6e74-4e38-8832-81d29cd50f41.1 Necrectomy in 2-nd day.jpg

Caption 2: Necrectomy in 2-nd day

P089 Treatment of «sofa injuries» 
D.O. Vagner ${ }^{1}$, E.V. Zinoviev'1, V.V. Soloshenko ${ }^{1}$, N.S. Chuvashev²

${ }^{1}$ St. Petersburg Institute of Emergency Care named after I.I. Dzhanelidze, SAINT-PETERSBURG, Russia

${ }^{2}$ North-Western state medical university named after I.I. Mechnikov, SAINT-PETERSBURG, Russia

Objectives: Smoking in bed or sofa coupled with alcohol intoxication is one of the causes of severe burns when a cigarette is dropped by the person while asleep. Due to alcohol intoxication the pain threshold decreases and consciousness is depressed. This is accompanied by the patient's long exposure to the fire with the formation of extensive and deep burns. In this case burn wounds are located in the body areas adjacent to the burned sofa. The term "sofa injury" is widely used to describe this multifactorial thermo-toxic injury

Methods: A retrospective study included patients with burns more than $10 \%$ TBSA. The main group consisted of 60 patients who got burned as a result of accidentally burning a couchwith a cigarette. The control group included 330 patients with burns of the same areas whichwere not associated with "sofa injuries".

Results: Sofa injuriesare more common in male than in female $(p=0.002)$. Alcohol intoxication significantly increases the risk of such injuries $(p=0.000)$. Simultaneous skin grafting after early escharectomy is possible in no more than $42 \%$ of cases due to extremely criticalcondition of such patients and the high frequency of subfascial lesion. Patients with a "sofa injury" need a longer ( $\mathrm{p}=$ $0.000)$ and more expensive $(p=0.05)$ treatment than those with burns in the control group. Mortality in this group is $53 \%$, which is significantly more than in any other form of thermal injury $(p=0.002)$.

Conclusion: Given the extremely high mortality and costs of treatment, prevention and optimization of treatment of "sofa injuries" is an important problem of emergency care.

Picture 1: https://www.eventure-online.com/parthen-uploads/89/9EBA/add_1_539819_6e45de8e2fc2-4a1c-ad9c-1ffeebeea371.1.jpg

Caption 1: Patient with 'Sofa injury'

P090 When should prophylactic antibiotics be used for ward based dressing changes?

\section{T] Challoner}

Queen Elizabeth Hospital, BIRMINGHAM, United Kingdom

Objectives: To ascertain whether all patients with significant burns $>10 \%$ TBSA should be offered prophylactic antibiotics prior to routine dressings changes to prevent transient bacteraemia, and does the bacteraemia lead to significant systemic effects.

Methods: Casenotes were reviewed from The Burn Centre at the Queen Elizabth Hospital, Birmingham, looking at which patients were at risk of bacteraemia, which patients received antibiotics routinely, and whether a postprocedure spike in temperature was relevant to systemic upset. This would hopefully define whether a standardised treatment protocol could be defined for these patients, or identify those at risk.

Results: Patients admitted between January 2018 and January 2019 were included, which included 40 patients undergoing 98 dressing changes. Prophylactic antibiotics were given in 15 cases (15.3\%). Temperature spikes likely caused by transient bacteraemia (without other identifiable sources of infection), occurred in $68 \%$ of dresssing changes.

This led to positive blood cultures in $8 \%$ of patients who required ongoing treatment for eradication.

Conclusion: Skin flora should be considered in dressing changes as those with positive bacterial loads are at increased risk of bacteraemia and systemic effects. Prophylactic antibiotics should be prescribed depending on sensitivities to prevent septic showers. A comparison should be made with theatre cases as anecdotally the rate of antibiotic prescription is higher in patients in a theatre setting.

P091 MANAGEMENT OF COMPARTMENTAL SYNDROME THROUGH NEXOBRIDR

ISA SAEZ AVILES, IFF FUENTES FERNANDEZ, JGJ GARCÍA JUARRAN, ASB Sánchez Balado, MUO UMAÑA ORDOÑEZ, CRA RECALDE ANDRES, ANT NOVO TORRES, ELB LORDA 


\section{BARRAGUER}

\section{ALICANTE GENERAL HOSPITAL, ALICANTE, Spain}

Objectives: The management of deep dermal circumferential burns at the level of the extremities is complex, especially at the acute moment of the injury. Establishing which is the best therapeutic option (enzymatic debridement Vs surgical debridement) is not always easy.

Methods: Circumferential burns on the extremities are likely to cause compartment syndrome during its evolution. At this point the use of the Nexobrid ${ }^{\circledR}$ is becoming a useful workhorse for the Burned units, avoiding escharotomy at the acute moment of the injury.

Results: We present the use of Nexobrid ${ }^{\circledR}$ in two cases with deep dermal burns at the extremities with favorable evolution, avoiding escharotomy at the acute time.

Conclusion: Nexobrid ${ }^{\circledR}$ has been proven to be a non surgical alternative for the removal of burn necrotic tissue. In our experience Nexobrid ${ }^{\circledR}$ could be a good option to avoid surgery.

P092 The use of enzymatic debridement in large burns two years of experience

IMINIC, E Vigato, M Governa

Azienda Ospedaliera Universitaria Integrata Verona, VERONA, Italy

Objectives: Modern era of treatment of burn injury stated with revolutionary approach of early surgical debridement followed by autologous skin grafting since early sixties of the last century. For many decades it's been a gold standard treatment. It's considered effective although not selective approach in terms of blood loss and vital tissue preservation.

We hypothesized that selective enzymatic debridement could have the positive outcome in terms of local control of wound healing and could decrease systemic inflammatory response.

Methods: Since February 2017 till February 2019 in our Burn Unit, we treated over 50 cases of partially deep and deep burns. Thihirty nine out of 50 was $>15 \%$ TBSA (15-85\%). Most of the patients (34 out of 39) was treated in BU or ICU. According to our protocol allograft skin grafting followed enzymatic debridement. Skin biopsy was performed before and after treatment. Three cases died for other complications and comorbidity.

Results: We respected the gold standard performing complete selective debridement within 1,4 days. Mean age was 54,1ys ( 1.1-96 ys). No blood transfusion was necessary after enzymatic debridement. Only one procedure was necessary for the majority patients (32 out of $39 \mathrm{pt}$ ). Twenty-ight out of 39 patients went to OR for skin grafting. For the burn wound completion there was a necessity of average 5,8\% autografts. Infection was well controlled with antibiotics therapy. Five cases of sepsis and one case of bacteremia was registered.

Conclusion: Regardless it a limited number of patient and only one year of experience, obtained results are encouraging. Rapid enzymatic debridement as a minimally invasive tool permitted to control burn wound not only on the local level but it seems like that has improved pro and antiinflammatory response in terms of better control of the SIRS. Further investigations will be necessary to confirm it.

P093 Functional evaluation after enzymatic debridement of intermediate burns in hands

\section{IR MARTINEZ-MENDEZ, C Corrales, A Gonzalez-Miranda, C Casado}

La Paz University Hospital, MADRID, Spain

Objectives: Surgical debridement of the burned hand may mean an important functional risk. Bromelain based enzymatic debridement has been reported to reduce the need for autografting as it leaves dermal remnants to allow sponataneous healing of partial thickness burns. This allows early rehabilitation which may have an important influence in eventual hand function. The aim of our study is to evaluate burn hand function after Nexobrid treatment and to compare it with healthy hand function.

Methods: Twelve prospective patients were evaluated during 2015 until March 2016 (4 females, 8 males), with an intermediate-deep burns in one of their hands. Burns were debrided using a bromelain based enzymatic product, without the need to be autografted during the follow up. Functional assessment were done using QuickDash test, Michigan Hand Outcomes Questionnaire and range of 
motion of the main hand joints. These tests were evaluated in the burned hand and in the healthy one.

Results: The average follow-up was 12 months, with an averaged QuickDash score in the burn hand was $2.15 \%$ with a range between $0 \%$ and $7.15 \%$, while the non-burn hand score was $0 \%$ in all patients. Michigan Hand Outcomes Questionnaire media score was $98,1 \%$ in the burn hand, with a range betweeen $92.3 \%$ and $100 \%$, in comparison with $99.43 \%$, and $98,34 \%$ and $100 \%$, in the non-burn hand. Either the Quick Dash or the MHOQ test scores reached the differences considered as Minimal Clinically important. Thumb opposition media range was $76 \%$ and $77.58 \%$ in the burn and healthy hand. Finally, proximal interphalangeal joint flexion range was $118.33 \%$ and $119.17 \%$ in the burn and healthy hand. None of these range differences were significant.

Conclusion: Bromelain based enzymatic debridement of intermediate burns in the hands with spontaneous healing allows an early functional recuperation, without differences with the healthy side in the questionnaires of functional assessment.

Picture 1: https://www.eventure-online.com/parthen-uploads/89/9EBA/add_1_540671_9fffeae1fdd2-4e5c-b317-42e21c7fe125.jpg

Caption 1: Deep dermal flame burn in a 47yo male. Preop, after removal of the debridement product and after 4 months.

P094 Efficacy of enzymatic debridement over elderly patients

IR MARTINEZ-MENDEZ, A Ojeda-Regidor, M Rubio, A Gonzalez-Miranda, C Casado, B. Pereira La Paz University Hospital, MADRID, Spain

Objectives: Bromelain based enzymatic debridement is an accurate and fast modality to debride buns but its indication in patients older than 65 is still controversial. The aim of the study was evaluate the efficacy of the debridement in older patients and compare its results to an historical cohort of patients treated with the standard of care.

Methods: A retrospective study was developed. Patients treated between 2015 to 2017 older than 65 y.o. and with less than $15 \%$ TBSA were collected. The control group was designed with an historical cohort from 2013 and 2014 when enzymatic debridement was not approved in our country. Both groups were homogeneous for TBSA, age, sex and inhalation syndrome. Epidemiological figures, length of stay, number of interventions and mortality was recorded. Statistical evaluation were done using $U$ Mann Whitney and $x 2$ tests from SPSS 21.0 Software.

Results: 80 patients were enrolled in the study, and divided in two groups: 60 controls and 20 cases. Average TBSA was $6.37 \%$, with a mean age of 78,1 y.o. The mortality ratio was $10 \%$, with same mean values for both groups. The time to complete debridement was $6,77 \pm 7,4$ days in the control group vs $2,15 \pm 1,7$ days in the experimental group. The average number of surgical interventions in the control group was $1,47 \pm 0,74$ and $0,75 \pm 0,71$ in the Nexobrid group. Despite these advantages, the length of stay were not statistically signficant, with $17,43 \pm 13,3$ days in the control group and $19,65 \pm 11,77$ days in the experimental one.

Conclusion: Bromelain based enzymatic debridement allows to reduce the number of surgeries over older patients, reducing the time until complete debridement of burns. No impact over length of stay was demostrated in this study.

P095 Lengyel P., Frišman E., Baran M.: Surgery to electrical burns

\section{P Lengyel, E Frišman}

Nemocnica Košice-Šaca a.s.-1.súkromná nemocnica, KOŠICE - ŠACA, Slovak Republic

Objectives: 3635 patients in years $2004-2018$ were hospitalized in our workplace with thermal injury, 127 of them were electrical burns $/ 3,49 \% /$.

Methods: The prefered goal of wound treatment was to give time space to tissues for spontaneous healing. In the acute phase of injury if there was necrotic tissue we performed necrectomy and reconstruction of the skin coverage by skin grafting or flap plasties. When high tension injury cases of mutilating electric injuries we were forced to perform amputation to gangraenous limbs.

Results: 152 surgical procedures were made to 89 pacients from the electrical burned patients, 38 patients healed without surgery. Of them in the group of men 58 needed some surgery $/ 62,36 \%$ / and 35 
healed spontaneously $/ 37,64 \% /$. If surgery was done, then 75 procedures were skin grafting $/ 67,6 \% /, 20$ were flap plasties $/ 18 \%$ / and 16 procedures were amputations / $14,4 \% /$. In the group of electrically burned children 27 needed some surgery $/ 90 \%$ / and 3 without operations /10\%/. 24 operations were skin grafting $/ 64,9 \% /, 8$ operations were flap plasties $/ 21,6 \%$ / and 5 amputations $/ 13,5 \% / .4$ women were electrically burned. Every one of them had once operation, 1 flap plasty and 3 skin grafting.

Conclusion: In the acute phase of treatment consists of excising devitalized tissue, appropriate wound care to prevent delayed infection, providing temporary and definitive coverage over vital structures.

P096 Combined chemical and flame burns due to an electronic nicotine delivery system: first report on neutralization of the chemical product, followed by enzymatic debridement

E.Y. Claes, E. De Wolf, H. Hoeksema, J. Verbelen, S. Monstrey

Ghent University Hospital, GENT, Belgium

Objectives: Since its release in 2007, the number of reports on injuries caused by explosion of electronic nicotine delivery systems (ENDS) has significantly increased.

Methods: A retrospective analysis of our burn database was performed from 2007 to December 2018 for injuries by ENDS. These events appear to result from lithium-ion battery overheating to the point of catching fire or exploding ("thermal runaway").

Results: Since 2007, two male patients were treated in our burn center for ENDS injuries. Their battery came into contact with metal objects that created an external short circuit which caused the "thermal runaway" in the pocket of the patient. This resulted in a combined chemical and flame burn. In both patients the upper legs were involved (TBSA 9\%) and in one patient also the fingers were involved. Burn depth was a combination of partial thickness burns with a Laser Doppler Imaging defined healing potential of over 21 days with clear indication for surgery. Despite the fact that enzymatic debridement is not recommended for use after chemical burns, in these two cases enzymatic debridement was successfully performed after neutralization of the chemical product by the use of a hypertonic solution: Diphoterine. Wounds were treated by conservative treatment till wound healing. Mean time to complete wound closure was 48 days (range 35 - 61). Despite this, no hypertrophic scarring was observed at follow-up. No clinical nor laboratory adverse events occurred, proving the safety and added value of this approach after ENDS burns.

Conclusion: In two patients off label use of enzymatic debridement was performed after combined alkali and flame burns. In literature, caution is recommended in exposing these alkali burns to irrigation with water. Although mineral oil is advocated, in future, Diphoterine could be recommended to eradicate the chemical substance, in deep burns followed by the use of enzymatic debridement.

P097 The use of Single Layer Dermal substitute in cover of post burn deep defect with exposed bone (case report).

HSuca

Faculty hospital Kralovke Vinohrady, PRAGUE, Czech Republic

Objectives: A classic algorithm for covering deep defects with exposed bone, muscle or tendon in acute reconstructive surgery is use of: DIRECT SUTURE - FULL-THICKNESS AUTOGRAFT - LOCAL FLAP - EXPANDER - DISTANT FLAP - FREE FLAP. With the arrival of dermal regenerative templates, there is a change in the classical algorythmic ladder, where dermal replacement opens up new possibilities for covering large deep defects with the consequent creation of a high quality skin cover. The use of a negative pressure wound therapy (NPWT) for pre-treatment is appropriate in the preparation of wound bed.

The poster presents the course of solving a complicated deep defect with exposed bone by a singlelayer dermal substitute in a 68-year-old patient with a history of colorectal carcinoma, haemicolectomy with stoma, partial resection of liver for metastasis, post actinotherapy, with hypertension and II.type diabetes mellitus, chronic smoker bronchitis.

Methods: The patient was admitted to Prague Burn centre with a flame burn of 50\%BSA IIb-IIIgr., injured in a suicidal attempt. Burned areas were treated with necrectomy and split thickness 
dermoepidermal autograft. A defect with exposed bone and non-vital periosteum formed in the area of the ventral tibia. After non-vital periosteum resection (size about $15 \times 3.5 \mathrm{~cm}$ ) the defect was resolved using a negative pressure wound therapy system for 20 days, and after formation of granulation tissue on the wound bed, a single layer dermal substitute, dermoepidermal split-thickness autograft and NPWT system were applied at one time

Results: Treatment resulted in a fully healed area with high-quality skin cover.

Conclusion: The use of dermal regenerative template can change the classic ladder of treatment of acute deep defect in problematic area and a complicated healing process.

P098 Contemporary options and methods for coverage and closure of burn wounds. Koller J., Bukovcan P., Sarkozyova N., Ferancikova N.

\section{Koller, P. Bukovcan, N. Nina, N. Ferancikova}

Comenius University Medical Faculty\& University Hospital, BRATISLAVA, Slovak Republic

Objectives: Main goals of extensive burn treatment include emergency procedures focused on life saving, infection prevention/treatment, and the use of methods which capable to reduce the time to heal of the open/freshly debrided and/or excised deep and extensive burn wounds.

Methods: There does exist a complexity of methods used to reach the above mentioned goals. Total burn care (Herndon 2012) includes individualized approach to each patient based on appropriate diagnostic methods, long-term intensive care, early removal of necrotic tissues, and the use of temporary skin substitutes for wound coverage replaced later by permanent ones for wound closure.

Results: Appropriate application of total burn care methods proved to reduce both morbidity and mortality of extensively burned patients. Shorter hospital stay, reduction of complications and earlier start of patients mobilization/rehabilitation did contribute to reduction of complications caused by infection and prolonged bed rest. Shorter time to heal the wounds followed by comprehensive aftercare and rehabilitation did reduce the occurrence of hypertrophic scars and functional problems as well.

Conclusion: Advances in intensive care methods, surgical methods of early removal of necrotic tissues, temporary coverage methods followed by different approaches to permanent closure of the open burn wound areas, and individualized approach to each burn victim proved to be, according to our opinion, the most important factors contributing to reduced mortality and shortening the time to heal of extensive deep burn wounds.

P099 Avoiding Skin Grafts: The Local perforator Flap in Cutaneous Defects Covering

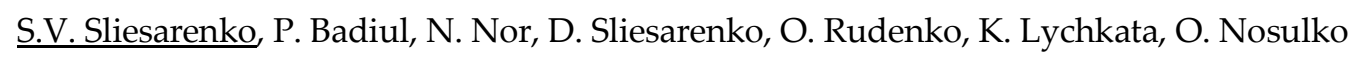

City Hospital \#2, DNIPRO, Ukraine

Objectives: Since its creation the first dermatome of 1939 skin grafts have become the most common method of wound closure. This method has long been called the "gold standard" of skin plastic. But, after surgery by attached split-thickness skin graft, does not fully recreate the lost skin. And the increased requirements of patients for the quality of life parameters require the improvement of plastic surgery in this aspect.

To improve the efficiency of wound closure surgery, we can select cases where it is possible to avoid skin graft transplantation. Instead, close the wounds by the local perforator flaps, which will restore the skin to the most similar to the lost.

Methods: In the first group (25 patients), the V-Y local flaps method was used. In the second group (28 patients) the Keystone local perforator flaps were used. In the third group (30 patients) skin grafts cover was used. Evaluated the rate of wound healing, the properties of the restored skin and the patient's quality of life.

Results: All patients received complete wound healing, in the first and second groups the wounds healed 2 times faster to compare with third group and the quality of the new skin was significantly better.

Conclusion: The local perforator flaps is a versatile and reliable reconstructive option for cover small or middle size wounds. This method provides restoration of the skin similar to the lost one, considerably superior in quality to the skin restored by engraftment of skin grafts. But limitations for 
local flaps is such as donor-site scarring or other skin problems. Sufficient experience the plastic surgeon of these modifications can guide to the appropriate reconstruction. Traditionally, split-thickness skin grafts were still as main method for large and extensive soft tissue Reconstructions, like on circular limb wounds.

Picture 1: https://www.eventure-online.com/parthen-uploads/89/9EBA/add_2_530753_fe3a83849add-4b81-8e3f-42a73033c6d9.JPG

Caption 1: The image of the wound before the reconstructive surgery

Picture 2: https://www.eventure-online.com/parthen-uploads/89/9EBA/add_1_530753_fe3a83849add-4b81-8e3f-42a73033c6d9.JPG

Caption 2: The image of wound intraoperative, after a radical excision and mobilization of local perforator islet keystone flap

Picture 3: https://www.eventure-online.com/parthen-uploads/89/9EBA/add_530753_fe3a83849add-4b81-8e3f-42a73033c6d9.JPG

Caption 3: The image of wound after healing, 3 weeks after surgery

P100 Hemi Abdomen Resurfacing with a Large Keystone Flap for Multiple Marjolin Ulcers with Field Change in a Burns Scar after 70 years

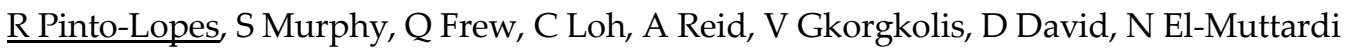

St Andrews Centre for Burns and Plastic Surgery, CHELMSFORD, United Kingdom

Objectives: Marjolin Ulcers (MU) refer to areas of malignant transformation in burn scars. These are usually squamous cell carcinomas but may be of rarer types such as basal cell carcinomas (BCCs), melanomas or sarcomas. These may appear after a variable period of time but also may be extensive and infiltrative in nature. We present a patient who had multiple burns scars over the anterior surface of her abdomen and left groin which were biopsy proven BCCs.

The degree of field change and close location of each burns scar to the other made individual excision and reconstruction challenging. We describe our management of this case and reconstruction of half the abdominal wall with a large keystone flap.

Methods: Complete oncological excision of the affected burns scars and MU was performed under general anaesthesia to the fascial wall. A large keystone flap was designed after marking out the keystone flap in Huger Zone 3. Perforators identifiable from the intercostal, subcostal region were preserved during the dissection and mobilised, ensuring viability of the flap. A separate MU in the left groin was resurfaced with a pedicled vastus lateralis flap with split thickness skin graft.

Results: The patient had complete clearance of the tumour infiltrated burns scars and the hemi abdomen was completely reconstructed with a keystone flap without issue.

Conclusion: Large keystone flaps in the trunk are useful for managing multiple burn scars with MU change. After oncological excision, resurfacing the hemi-abdominal wall with a flap provides the best outcome if further radiotherapy is required.

P101 Burns injures caused by electricity treated with dermal regeneration template

\section{B.C. Certic}

Clinic for burns, plastic and reconstructive surgery, Clinical Center, Serbia, BELGRADE, Serbia

Objectives: To demonstrate how in absence of adequate donnor regions large and massive defects and exposed parts of bones can be treated using dermal regeneration template.

Methods: This is a case report of a 27 year old patient with extensive burns of multiple body regions from electricity treated at the Clinic for plastic and reconstructive surgery, Clinical Center Serbia, Belgrade.

Results: This paper presents a 27 year old man with extensive burns of body regions (several places of entrance and exit) from electricity. The most severe defects covered the anterior and lateral side of the left thigh and exposed part of femur bone, right gluteus, perianal and sacral regions, left low leg and left foot. He was initially treated conservatively with $\mathrm{HBO}$ and all other substitution therapy. When general condition of the patient had been stabilized the surgical treatment in several phases started. In absence of donor regions and because of large and massive defects and exposed part of bone femur, 
dermal regeneration template was applied for the first time in Clinic for Burns, Plastic and Reconstructive Surgery, Clinical Centre Serbia, Belgrade. Dermal regeneration template use in this case enabled me to save patient's leg instead of amputating it. At the same time we attained better functional and aesthetic outcome for the patient. The patient was threaded for 76 days and 11 operations were done. After two years of completing the treatment the patient has no functional failure and he has very good aesthetic result.

Conclusion: We can conclude that adequate and early reconstruction of extensive defects after electrocution with application of dermal regeneration template with autografts and as well as eary rehabilitation should be the primary goal of treatment.

Picture 1: https://www.eventure-online.com/parthen-uploads/89/9EBA/add_2_538957_93bac87554c6-4b19-b66d-18d8c379de1a.JPG

Caption 1: Postoperative results of covering lateral tight and exsposed femur using dermal regeneration template

Picture 2: https://www.eventure-online.com/parthen-uploads/89/9EBA/add_538957_93bac87554c6-4b19-b66d-18d8c379de1a.jpg

Caption 2: Large defect of posterior thigh, gluteus, perianal region.

Picture 3: https://www.eventure-online.com/parthen-uploads/89/9EBA/add_1_538957_93bac87554c6-4b19-b66d-18d8c379de1a.jpg

Caption 3: Postoperative results.

\section{P102 RECONSTRUCTIVE SURGERY OF UPPER EXTREMITY AFTER THERMAL BURNS - GUIDELINES OR EXPERIENCE}

\section{A. Stritar, M. Mikša, L. Emeršic, A. Arhar}

University Medical Centre Ljubljana, LJUBLJANA, Slovenia

Objectives: Hand burns hold a special place in the field of burn care, even though the surface area of the hand comprises approximately $1 \%$ TBSA. This is due to their anatomical, functional and aesthetic characteristics. Surgical treatment is a priority in these types of burns, especially on the dorsum of the hand and fingers.

Methods: Early tangential excision is the method of choice for treating deep dermal and subdermal hand burns. In these cases surgery yields considerably better functional and aesthetic outcomes, as opposed to conservative therapy. Consequently, hands are given priority over other parts of the body in surgical interventions.

Physiotherapy and rehabilitation play a very important role in the treatment of hand burns. Functionality of the hand is of paramount importance for the quality of life of burn patients. Some general rules apply for the treatment of hand burns, i.e. individualised approach, careful patient selection and operative therapy based on the principles of the reconstructive ladder.

Results: Patient's psychophysical state and participation are undoubtedly important; they have to be monitored in addition to providing the patient with objective information regarding their treatment. The treatment should be opportune and carefully planned in regard to the expected timeline as well as to the prediction of the end result. Postoperative follow-up is lengthy, several operations may be required and the rehabilitation programme completes the whole treatment.

Conclusion: Guidelines and experience occasionally differ. The former primarily protects logistic and forensic facts, while the latter expresses operative, surgical and patient's outcomes. Nevertheless, hand reconstruction, be it primary or late, should abide by the protocol without sudden changes or improvisation, while being in accordance with the expected timeline and burn scar maturation.

\section{P103 FACIAL BURNS AND ENZYMATIC DEBRIDEMENT PRELIMINARY CASES}

\section{J MINIC, E Vigato, $\underline{\text { M Governa }}$}

Azienda Ospedaliera Universitaria Integrata Verona, VERONA, Italy

Objectives: Facial burns represent a big challenge even for more talented and expert surgeons.

Traditional surgery, dermabrasion or water jet method can be useful, but definitely more aggressive and less selective than enzymatic debridement, with additional necessity of operating 
theatre to perform the procedure.

Enzymatic debridement as a novel approach allows the removal of burn eschar on early basis admission in 4 hour application, respecting the remanences vital structures, especially dermis, fundamental for scarless epithelisation.

Methods: From February 2017 in our Burn Unit we started performing enzymatic debridement on our burn patients. We treated 6 patients (out of 26 patients) with facial burn injury. Most of them had other burn injuries including inhalators one.

Results: All the patients were treated within 36 hours after admission meanly (0-72 hours). Patients were four man and two females, mean age 36,25 (1,5 - 53 age) years old. They were mainly affected with $19,3 \%$ TBSA\% (3-37\%) burn injuries and some of them as well with inhalation injuries. None of them needed operating theatre for enzymatic debridement procedure. Allo transplants were applied afterwords. No blood transfusion was necessary. One out of six patients needed skin grafting due to a sepsis and one out of 6 patients had a serious damage of nose structure. The spontaneous wound healing was present within 14-20 day after injury.

Conclusion: These preliminary experiences encourage to perform enzymatic debridement on early basis admission like surgical procedure, permitting preservation of vital structures, reducing functional and cosmetic sequelae.

\section{P104 LOCAL FLAP RECONSTRUCTION OF DEEP HEAD BURN}

\section{$\underline{\text { Y Zayakova }}^{1}$, M Argirova ${ }^{2}$, B Tsvetkov ${ }^{1}$}

${ }^{1}$ MBAL-Varna, MMA-Sofia, VARNA, Bulgaria

2UMHATEM „N.I. Pirogov „,, Sofia,, SOFIA, Bulgaria

Objectives: The objective of this article is to present and analyze the results from the use of local flaps for the reconstruction of deep head post burn defects.

Methods: The study presents reconstructive surgeries performed on 23 patients with head defects resulting from deep burn and involving different areas and structures of the scalp and calvaria. The localization of the injuries are as follows: frontal $(n=7)$, occipital $(n=5)$ and central $(n=9)$ parts of the head. The size of the defects varied between $>20 \mathrm{~cm}^{2}<105 \mathrm{~cm}^{2}$.In two patients the defect involves almost half of the scalp. There was available good quality tissue around the wounds in all cases. The defects were treated with local flaps.

Results: Patients' recovery was quite quick, without hematoma, seroma or infection. All flaps completely survived except for one advancement flap where total skin necrosis occurred.

Conclusion: In cases of deep head burn and good surrounding tissue our preferred reconstructive method is the application of local flaps. They provide stable soft tissue coverage and quick recovery of the patients. The operative time is relatively short and the aesthetic and functional results quite satisfying.

\section{P105 Surgical needling in scar care}

\section{T Rose, PMF Francois}

Military Hospital, BRUSSELS, Belgium

Objectives: Using surgical needling with a $3 \mathrm{~mm}$ dermal roller give us a supplemtary tool in the treatment of burn scares

Methods: Presentation of the surgical planning and procedure, after care and results in our burn care practice

Results: We will share our experience in using the $3 \mathrm{~mm}$ surgical roller in scar care

Conclusion: Surgical needling with the $3 \mathrm{~mm}$ dermal roller is an supplementary techniques in treating scars

P106 Case report: Burn scars of 9\%TBS in different areas of the body on a female patient treated with four (4) different methods.

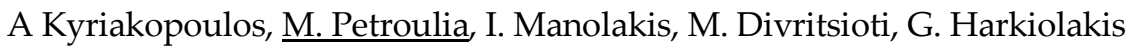


Evaggelismos Hospital of Athens, ATHENS, Greece

Objectives: Educational case report: Presentation of the four (4) different most important methods of treating successfully old burn scars simultaneously on one person (Female 58 years old with 9\%TBS old burn scars).

Methods: Triamsinolone acetonide use, Z-plasty, expansion of healthy skin to excise adjacent skin scar, full excision of the scar and cover with INTEGRA ${ }^{\mathrm{TM}}$ DOUBLE LAYER ${ }^{\mathrm{TM}}$ and skin graft. All these methods used harmonically on one female patient, non smoker, after consultation and singed consensus.

Results: Excellent results on all areas after 4 different surgeries with general anaesthesia in 6 months total time, excellent aesthetic and functional result. Improvement of the physiological status of the patient

Conclusion: Plastic surgery reconstructive ladder must be used in burn scars according to the guidelines in every patient, plastic surgeon should have no fear when combining harmonically different methods if planned correctly and have the patient appropriately consulted.

\section{P107 SKIN GRAFTING BY DR. MENZUL}

$\underline{\text { V.A. Menzul }}{ }^{1}$, A.E. Mordyakov², V.S. Borisov³, A.S. Kovalev'1, A.L. Levchuk4, T.I. Ignat'ev', A.V. Sachkov ${ }^{3}$

${ }^{1}$ The Main Military Clinical Hospital, Balashiha, Russia, MOSCOW, Russia

${ }^{2}$ Central City Clinical Hospital, ULYANOVSK, Russia

${ }^{3}$ Sklifosovsky research Institute for Emergency Medicine, MOSCOW, Russia

${ }^{4}$ National Medical and Surgical Center named after N.I. Pirogov, MOSCOW, Russia

Objectives: To improve the cosmetic results of mesh skin grafting.

Methods: The newest approach to the economical method of skin grafting using mesh grafts is autodermoplasty using a skin mesher, developed and patented by dr. V.A. Menzul in 2016 as "Method of preparation of the graft, skin meshers for its implementation, and a method for treating burn wounds" (Patent No. 2594446, 2016, RU; PCT WO 2017/213534 A1). This kind of skin grafting combines the advantages of skin grafting a mesh grafts with a non-perforated grafts. An important advantage of the new skin mesher is that, when used, it becomes possible to cut the diamond-shaped cells with a bridge in the center, which, while maintaining the total area of the graft, decreases the areas subject to epithelialization in each cell. In addition, the bridges in the cells are an additional source of tissue regeneration and epithelialization. This skin mesher has variants with different ratios of perforation 1:3, 1:4, 1:6. Taking graft and resection of the granulation tissue from the wound bed prior to skin grafting was performed using the disk electric dermatome of the original design. Treatment of donor sites and wounds with transplanted skin was carried out with the help of original film dressings.

Results: The application of a skin grafting using Dr. Menzul's skin perforator showed good clinical and cosmetic results (no pronounced "waffle" pattern at the transplant site).

Conclusion: Consequently, this novel type of skin grafting with Dr. Menzul's skin mesher is a promising method and a worthy alternative to skin meshers that do not create a bridge inside the graft cells.

P108 Use of acellular dermal substitute in treatment of skin contractures after burn injury - case presentation

\section{Miksa, $\underline{\text { A Stritar }}$}

University Medical Centre Ljubljana, LJUBLJANA, Slovenia

Objectives: The process of healing is especially important after burns of the upper extremities. In spite of timely and thorough primary and surgical care, the result is often unpleasant in the case of deep burns and appears in scar formation and skin contractures. Treatment is conservative, followed by surgical management. When performing surgical procedures, the concept of a progressive climb from simple to complex starting on the lowest rung and moving up until a suitable technique is arrived at, should be used.

In recent years, a number of dermal substitutes have been emerging, which are already part of the 
reconstructive ladder. Most of them are used individually, and some of them can be used in combination with partial skin grafts to improve the properties of the skin. In the previous 2 years we treated 10 patients with contractures of upper extremities, using acellular dermal matrix in order to restore the quality of skin.

Methods: Scar excision and reconstruction was performed with dermal substitute as a template for split-thickness skin graft. We evaluated visual appearance with photo documentation preoperative and 7 days after reconstruction.

Results: In all cases, the graft adhered completely. All 10 patients had better skin laxity after surgery and better mobility. We also documented thicker and more mobile dermis compared to nonsurgical area of scarring tissue.

Conclusion: The presented cases illustrate one of the possibilities in which skin substitutes combined with skin grafts are used to improve post burn scarring and mobility as well as better aesthetic and functional outcome.

P109 Application of synthetic bi-layer dermal matrix in severely burned child - a case study

A Ziola ${ }^{1}$, AM Bulandra ${ }^{2}$, T Koszutski

1Uppersilesian Child Health Center, KATOWICE, Poland

${ }^{2}$ Medical University of Silesia, KATOWICE, Poland

Objectives: Treatment of severely burned children is a serious clinical problem. The priority is to treat the burn disease and close the burn wound as soon as possible. The best treatment method is the early excision of necrosis and the coverage of burn wounds with autologous skin grafts. In cases of extensive burns, it can be difficult due to the deficit of the donor areas.

Methods: A case of a 3-years old severely burned child is presented. As a result of ignition of the seat in which the boy was fastened in the car, he suffered extensive third and fourth degree burns of the dorsal surface of the body - at least $47 \%$ TBSA. The necrosis was deeply resected during the first 72 hours after injury. A half of the burned area was covered by the autologous skin grafts, but the deficit of donor areas caused the need to close the rest of wounds in a different manner. A be-layer synthetic dermal matrix was applied to temporarily close the wounds. Later, the matrix was the basis for autologous skin transplantation.

Results: The procedure allowed to obtain the time needed for donor sites healing before subsequent skin donation. It also caused the restoration of the damaged layer of the dermis, which improved the functional and cosmetic effect of the treatment.

Conclusion: In presented case application of synthetic skin matrix gave a good final result. However, the best way to burn wound closure is primary autologous skin grafting, the matrix application should be considered in special circumstances like insufficient donor area.

P110Combination of allodermis and VAC therapy in hand surgery after burn injury

MB Baran

Hospital of Kosice-Saca, KOSICE, Slovak Republic

Objectives: Our objectives were to reconstruct dorsal skin compartment of hand disabled by massive hypertrophic scars after burn injury.

Methods: We used human cadaver acellular allodermis in combination with VAC intermittent low-suction pressure therapy and dermoepidermal skingrafting.

Results: Mobility with almost full flection in MTP, PIP and DIP joints were achieved without recidive of hypertrophic scarring.

Conclusion: Acellular allodermis allow placing of elastic structure between epidermis and subcutaneous structure with minimal expenses and combination with VAC therapy helps increase vitality of dermoepidrmal skin grafts.

P111 Succesful use of Integra $\mathbb{R}$ in multimodal treatment of extensive hypergranulations in a 14year-old polytrauma female patient after electrical burns 
A Munjiza ${ }^{1}, Z_{Z}$ Godec $^{1}$, A Gveric-Grginic ${ }^{1}$, M Domijan $^{1}$, M Matoic $^{1}$, M Romcevic $^{2}, \mathrm{H}_{\text {Tomicic }}{ }^{1}$

${ }^{1}$ Sestre milosrdnice University Hospital Center, ZAGREB, Croatia

2Polyclinic for cardiovascular Disease Prevention and Rehabilitation, ZAGREB, Croatia

Objectives: A 14-year-old female patient was admitted to our ward six months after being treated in another health facility. The patient was injured by electrical arch of $25000 \mathrm{~V}$ and sustained $3^{\text {rd }}$ degree burns affecting 50 percent of TBSA as well as a spleen rupture due to a fall from the height. Urgent splenectomy was performed. Extensive infected hypergranulations in the perineal region, back and lower extremity caused delayed healing.

Methods: This problem required a multimodal approach. After meticulous debridement of hypergranulations and wound conditioning, Integra ${ }^{\circledR}$ was applied in the lower extremity and perineal region. The patient was closely monitored for signs of bacterial infection so nine days after surgery a silicon layer of Integra ${ }^{\circledR}$ had to be removed. STSG meshed graft 1:1.5 was applied after removal of silicon layer and wound conditioning. Skin grafting was repeated in the areas that had not been successfully grafted, with additional usage of PRF (Platelet-rich fibrin) and CEA (Culture epithelial autograft).

Results: Preoperative wound conditioning and simultaneous use of antibiotics allowed a successful use of Integra ${ }^{\circledR}$ in spite of multiresistant Pseudomonas aeruginosa colonization. After 70 days of treatment at our ward, complete epithelization was achieved. Restoration of anatomy and function, with significant improvement in the range of motion of involved joints, was achieved in a relatively short period of time.

Conclusion: The beneficial effect of Integra ${ }^{\circledR}$ was possible because the biological response of the young patient was so quick that the dermis was restored in only 9 days and the injured lower extremity was similar to the other in both volume and shape. Simultaneous application of different methods, including proper nutritional support, speeded up the healing time.

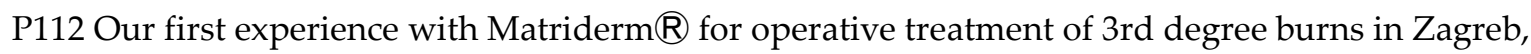
Croatia

A Munjiza ${ }^{1}, Z_{\text {Z }}$ Godec $^{1}$, M Matoic ${ }^{1}$, M Domijan ${ }^{1}$, A Gveric-Grginic ${ }^{1}$, M Romcevic $^{2}$, H Tomicic $^{1}$

'Sestre milosrdnice University Hospital Center, ZAGREB, Croatia

${ }^{2}$ Polyclinic for cardiovascular Disease Prevention and Rehabilitation, ZAGREB, Croatia

Objectives: After 10 years of worldwide awareness of beneficial effects of Matriderm ${ }^{\circledR}$ for operative treatment of 3rd degree burns, we finally have the opportunity to use it in our everyday practice. First results seem to be encouraging and we present our two cases.

Methods: We used Matriderm®for the first time in a 53-year-old female who sustained a 3rd degree scald burn of the lower leg. After necrectomy, it was evident that problematic regions were the popliteal area and denuded portion of the Achilles tendon in the extent of $7 \mathrm{~cm}$. Necrectomy encompassed the entire posterior area of the lower leg. One sheet of a $1 \mathrm{~mm}$ thick Matriderm ${ }^{\circledR}$ was used and covered with a STSG meshed graft (1:1.5), and then dressed with Vaseline gauze and hydrofiber dressing enriched with silver. In our second case, we used Matriderm ${ }^{\circledR}$ in a 64-year-old female who sustained a 3rd degree scald burn in the Achilles tendon region. After necrectomy, there was a $5 \mathrm{~cm}$ large denuded portion of the Achilles tendon, so we also applied $1 \mathrm{~mm}$ thick Matriderm ${ }^{\circledR}$, covered it with a STSG and dressed it in the same way as in the first case.

Results: First control showed excellent take in both cases. In the first case, only a minor area above the tendon was problematic, so we decided to treat it with hemoglobin spray and NPWT during 4 weeks. With early physical therapy, range of motion was more than satisfying, as well as aesthetic results.

Conclusion: In comparison to using only STSG, the use of Matriderm ${ }^{\circledR}$ in the treatment of 3 rd degree burns for very demanding areas resulted in better aesthetic and functional outcomes. The main advantage was its compatibility with other supporting methods such as NPWT.

P113 Pediatric Burns Treated with Ambulatory Way with Excellent Results with Epifast Cultivated Skin Graft 


\section{AMS MASSE SANCHEZ, GUS HERNANDEZ}

\section{MEXICAN SOCIAL SECURITY INSTITUTE, GUSTAVO A. MADERO, Mexico}

Objectives: Provide adequate, rapid and safe treatment for pediatric burn patients of 1st and 2nd degree who can be managed in the outpatient clinic, for which we use Epifast cultured skin grafts.

Methods: There are several cases of pediatric patients who were managed on an outpatient basis since in their case they did not meet the requirements to be hospitalized. In some cases the patient was subjected to anesthesia and after the surgery was discharged, in other cases the Epifast was placed, holding it with micropore and bandage and immobilization and they were discharged with the indication of being at home at home.

Results: After 8 days they returned to the outpatient clinic the epiplast of the wound was removed, with total epithelialization in most cases, in adolescent patients the management was more difficult, requiring 2 or 3 times the application of Epifast.

Conclusion: The management of 1st and 2nd degree burns in pediatric patients with Epifast cultivate skin grafts, is an excellent option since with a single procedure an excellent result was obtained, I do not leave aesthetic or functional problems, I diminish the discomforts and they were not reported. No rejection or infections.

P114 Improving Cosmetic Results of Frontal Hairline Reconstruction Through a Combination of Lambda Incisions and Z-Plasties in Pre-Expanded Forehead and Scalp Flaps in Children

\section{GOFFINET}

\section{CHRU Nancy, VANDOEUVRE-LES-NANCY, France}

Objectives: Skilled surgical teams have had good results reconstructing defects involving up to $50 \%$ of the scalp by using pre-expanded flaps. Forehead reconstruction is more difficult, with special problems including correction of eyebrow position and difficulty achieving adequate expansion in defects involving more than $25 \%$ of the area due to risk of paralysis of the frontalis muscle. Natural anterior hairline reconstruction especially remains a challenge, as it is usually used as the border of the reconstruction, at the expense of the natural gender-specific appearance of the hairline. The purpose of this paper is to present a technique in improving cosmetic hairline reconstruction by systematic combined Z-plasties and Lambda incisions in free-style forehead and scalp pre-expanded pedicled flaps adapted to the individual patient.

Methods: A retrospective review was conducted of consecutive patients of the lead author undergoing pre-expanded flap reconstruction for forehead and/or scalp defects involving the hairline between November 2009 and January 2016. Clinical data, operative details, psychosocial burden, and aesthetics impairment evaluations by distance method were collected.

Results: 2 patients with severe forehead or scalp defects of separate etiologies underwent total expansion protocols in the study. The periods of expansion were 13.5 and 21 weeks respectively. All details concerning implants, flap characteristics, and adjunct procedures are reported, as well as pre-, intra- and post-operative images.

Conclusion: Based on our results in pediatric cases with disparate mechanisms of injury and defects, we find that it is possible to utilize a novel combination of two known surgical techniques in order to improve the cosmetic results in cases of scalp and/or forehead reconstruction. In such a complex area, decreasing psychosocial impairment by restoration of symmetry and the natural limit of the hairline is of paramount importance and can be achieved reproducibly using the techniques described here.

Picture 1: https://www.eventure-online.com/parthen-uploads/89/9EBA/add_3_541877_328aa8a5eb9b-4a23-9f45-9b80504d18cf.png

Caption 1: Pictures reports the case 2 in the frontal (a) and lateral (b) view at the first consultation.

Picture 2: https://www.eventure-online.com/parthen-uploads/89/9EBA/add_1_541877_328aa8a5eb9b-4a23-9f45-9b80504d18cf.png

Caption 2: This is the detail of the second iterative expansion a non-expanded peninsular flap supported by a pre-expanded flap design posteriorly

Picture 3: https://www.eventure-online.com/parthen-uploads/89/9EBA/add_2_541877_328aa8a5- 
eb9b-4a23-9f45-9b80504d18cf.png

Picture 4: https://www.eventure-online.com/parthen-uploads/89/9EBA/add_541877_328aa8a5eb9b-4a23-9f45-9b80504d18cf.png

P115 Extensive Electrical Burn Injury: Long Term Outcome Involving Head, External Genitalia and Inguinal Area

RFM Lumbuun, A Wardhana, I Aulia

Cipto Mangunkusumo Hospital - Faculty of Medicine, Universitas Indonesia, JAKARTA, Indonesia

Objectives: Electrical burn injury is devastating among other burn injury. The involvement of the head, external genitalia, and inguinal area are rare comparing to the limbs. Although the surgical treatment is well established for the limbs, the management of genital lesions remains challenging

Methods: We did a retrospective review of electrical burn injury patients admitted in our burn center from January 2016 to December 2018. We analyzed patient demographics, persent total body surface area, region wise distribution, etiology, the problems encountered and surgeries that have been done, and outcome of the patients

Results: 69 patients out of 472 patients were admitted due to electrical burn injury. $94 \%$ of them is men. We did total 64 surgeries in 35 electrical burn injury patients. Serial debridement of wounds were performed before we did reconstructive surgeries. Surgeries that have been done including debridement, amputation, split skin graft, and loco-regional flaps, based on the reconstructive ladder.

Conclusion: Extensive electrical burn injury with involvement of head, external genitalia, and inguinal area represent a devastating injury which require timely approach. Adequate staged surgeries were needed to receive good outcome.

Picture 1: https://www.eventure-online.com/parthen-uploads/89/9EBA/add_1_542123_3bd93ca03aa6-492d-bb25-214fc8f564d1.png

Picture 2: https://www.eventure-online.com/parthen-uploads/89/9EBA/add_542123_3bd93ca03aa6-492d-bb25-214fc8f564d1.png

Picture 3: https://www.eventure-online.com/parthen-uploads/89/9EBA/add_3_542123_3bd93ca03aa6-492d-bb25-214fc8f564d1.png

Picture 4: https://www.eventure-online.com/parthen-uploads/89/9EBA/add_2_542123_3bd93ca03aa6-492d-bb25-214fc8f564d1.png

P116 Glabrous Skin Graft for Bilateral Full Thickness Plantar Burns

AM Malan, A Vorster

Tembisa Hospital, TEMBISA, KEMPTON PARK, South Africa

Objectives: Glabrous burns are a unique subtype of burns and are challenging to manage due to the unique histological, functional and aesthetic properties. Following the plastics principle of "replacing like with like tissues", we opted to trial a glabrous skin graft in order to maximise function, minimise disfigurement, restore appearance and normal anatomical structure.

Methods: The patient underwent a staged reconstruction of the plantar surfaces. The first surgery consisted of an autograft from the left thigh to the plantar surface for wound bed preparation. During the second surgery the non glabrous autograft from the left foot was peeled off. Glabrous skin was harvested from the right foot with a dermatome at $0.45 \mathrm{~mm}$. The donor skin was meshed 1:3 and was clipped in placed onto the wound. The wound was dressed with paraffin gauze and dry dressings. Wounds were exposed on day 5.

Results: At one month post graft the wound is completely healed with hyperpigmentation and peeling off hyperkeratotic skin. Hyperpigmentation can be attributed to the migration of melanocytes from the initial autograft. There was adequate restoration of function and reasonable aesthetics. Donor sites are completely healed. At 5 month follow up the wound is smooth with recontouring and fill out of the recess, return of sensation and exposure to surfaces without marked sensitivity. Donor site wounds are no longer distinguishable. At the periphery of the non glabrous skin graft on the right foot we noted ealry hyperkeratotic scar formation. Patient complained of intermittent parasthesia of the 
right foot. Nil complaints of the left foot.

Conclusion: The histological differences give glabrous skin its characteristic function, allowing for increased durability to pressure and shear force, as well as ability to adapt to changes in surface contours. These highlight the advantageous indication for using glabrous skin. The use of nonglabrous skin complicates with hyperkeratotic scarring, contractures and subfibrotic tissue. This case reports demonstrates successful use of glabrous skin for treatment of full thickness plantar burns and despite misconceptions there was no donor morbidity. Thus we conclude that replacing like with like tissue yields superior outcomes resembling virgin skin anatomically, functionally and aesthetically.

Picture 1: https://www.eventure-online.com/parthen-uploads/89/9EBA/add_2_558226_3a6134d44362-4f1b-97d1-133449c6d63d.jpeg

Caption 1: Post first surgery

Picture 2: https://www.eventure-online.com/parthen-uploads/89/9EBA/add_1_558226_3a6134d44362-4f1b-97d1-133449c6d63d.jpeg

Caption 2: Pre operative bilateral plantar wounds with foot arches and weight bearing areas

Picture 3: https://www.eventure-online.com/parthen-uploads/89/9EBA/add_558226_3a6134d44362-4f1b-97d1-133449c6d63d.jpeg

Caption 3: 5 months post operative

Picture 4: https://www.eventure-online.com/parthen-uploads/89/9EBA/add_3_558226_3a6134d44362-4f1b-97d1-133449c6d63d.jpeg

Caption 4: 1 month left foot post operative

P117 A full-thickness skin construct made of a collagen hydrogel strengthened by a fibrin-modified nanofibrous membrane

I Pajorova, M Bacakova, A Broz, M Travnickova, L Bacakova

Institute of Physiology CAS, PRAGUE 4, Czech Republic

Objectives: For many years, a three-dimensional (3D) collagen hydrogel has been used for embedding the fibroblasts to provide them a physiological 3D environment. However, the stiffness of tissue-engineered skin analogues based on collagen hydrogels is insufficient. In order to improve the mechanical properties of a two-layer skin construct, we stabilized the collagen hydrogel by fibrinmodified nanofibrous membrane.

Methods: Nanofibrous polylactid acid (PLA) membranes were modified with fibrin homogenous mesh. Fibrin-modified membranes were seeded with human dermal fibroblasts or adipose-derived stem cells (ASCs). After few days, collagen hydrogel was applied on the surface of fibrin-modified membranes preseeded with fibroblasts or stem cells and then primary keratinocytes were seeded on the surface of collagen hydrogel. Cell proliferation, migration and morphology were evaluated by MTS assay and by microscopy.

Results: We observed that the fibrin homogenous mesh enhanced the attachment and growth of fibroblasts and ASCs and additionally it supported their migration upwards into the collagen hydrogel. On top of that, the contraction and shrinkage of the collagen hydrogels by the traction forces of fibroblasts were minimalized by their gradual migration from membrane into the hydrogel. The keratinocytes on the top of the collagen construct formed a basal layer of highly dividing cells, and a suprabasal layer.

Conclusion: The construction of full-thickness skin substitutes that are similar to natural tissue is a promising approach to deep skin wound repairs. We can conclude that the collagen hydrogel reinforced by synthetic membrane provides a suitable microenvironment for the skin cells with sufficient mechanical properties for possible clinical applications.

P118 Treatment of 2a-b degree burns of the face with a alloplastic nanocellulose-based epidermal substitute (epicite hydro)

\section{Nietzschmann, F Siemers}

BG-Klinik Bergmannstrost Halle, HALLE, Germany

Objectives: The treatment of 2a-b degree facial burns ist carried out conservatively in our burn 
centre. Until now we used hydrocolloid bandages until healing. For 1.5 years we have treated 50 patients with epicite hydro to find out about the feasibility and possible benefits compared to our former treatment procedure.

Methods: On the day of admission, after the ususal admission procedure in the form of a wholebody washing/ shaving and dermabrasion, the epicite hydro was applied instantly to the burned area instead of the previously used antiseptic moist dressings.

The previously performed change after 24-36 hours to a hydrocolloid dressing was no longer necessary. The applied epicite hydro-facial mask remained on the face and was moistened daily with an antiseptic solution until the 5 th day in the case of large $2 b$ degree areas. In the case of $2 a$ degree burns, the epicite hydro remained on the face with no additional action.

The epicite hydro was only changed if there was a strong feeling of tension. In most cases it remained unchanged until complete drying after more than 10 days.

Results: With this new treatment regime, fewer dressing changes were necessary. The exudation which occurredunder the hydrocolloid dressings, and which was perceived as unpleasnat, was eliminated.

Conclusion: Later surgery was not necessary for any patient.

P119 Evaluation of Glehnia littoralis extract Loaded Poly(vinyl alcohol)/Pectin Hydrogel for Wound Healing

\section{$\underline{\text { YJ Park }}$}

Chosun University Hospital, GWANGJU, South Korea

Objectives: Glehnia littoralis (Umbelliferae) is a traditional medicine used in Korea, China, and Japan to treat the immune related diseases. However, its anti-bacterial and wound healing activities remain to be defined. In this present study, the hydrogel wound dressing was made using G. littoralis extract (GLE), which have been proven to have strong antimicrobial activity, and their wound healing efficacy was investigated through rat clinical experiment.

Methods: 1. In Vitro study of antimicrobial reagents

GLE compounds were prepared according to the recipe reported in the previous literature. Cytotoxicities of the treated fibroblast cells were observed by MTT assay.

2. In Vivo study

A total of 18 male Sprague Dawley rats enrolled. After rats' back skin wounded by contact burn, the hydrogel wound dressing was made using GLE, which have been proven to have strong antimicrobial activity especially against S. epidermidis, and their wound healing efficacy was investigated through visual and histopathologic finding.

Results: In vitro cytotoxicity tests showed that the developed GLE-PVA:PET hydrogel was noncytotoxic. Antibacterial activity tests revealed that the antibacterial activity of GLE in the hydrogel did show a significant difference compared with that of the control hydrogel. Furthermore, in vivo wound healing tests showed that the rate of wound healing was greatly elevated with the rapid reepithelialization in the GLE-PVA:PET hydrogel group.

Conclusion: The results obtained showed that GLE-PVA:PET hydrogel has good wound healing and antibacterial properties. These findings validate the use of this plant in the medical field for the treatment of wounds.

Picture 1: https://www.eventure-online.com/parthen-uploads/89/9EBA/add_1_533524_750cce6130be-446a-a604-afd9b22b2a01.jpg

Caption 1: Clinical Rat Experiment

P120 The use of Epiprotect $\mathbb{R} 2117$ in the treatment of superficial to mid dermal facial burns

\section{S. Stevoska, $\underline{\text { A. Stritar }}$}

University Medical Center Ljubljana, LJUBLJANA, Slovenia

Objectives: Superficial and mid dermal facial burns do not necessarily require operative treatment. We treat them either stationary or in outpatient clinic. The goal of treatment is to provide an appropriate environment for wound healing, to prevent infection and to reduce pain, thus achieving a good 
functional as well as aesthetic outcome. In the initial care, every fresh burn wound, regardless of its depth, is cleaned and in the aseptic conditions, an absorptive compress is installed. After few days of initial care, there is a variety of new dressings currently available for usage, such as often used hydrocolloid gels. In our cases we used a new microbial cellulose dressing, Epiprotect ${ }^{\circledR}$, evaluated the outcome and compared it to hydrocolloid gels.

Methods: In the period between October 2018 and March 2019, 5 patients, mostly male, average age 47 years, were treated with Epiprotect ${ }^{\circledR}$. All burn wounds were superficial and mid dermal facial burns and accounted for more than 5\% of STP. All patients were hospitalized and regularly monitored. The dressing was placed under aseptic conditions after few days of initial care and left attached until it self-released from skin after the wound re-epithelization. No secondary dressing was needed. Following the demarcation of Epiprotect ${ }^{\circledR}$, the use of nourishing ointments was advised.

Results: Satisfactory aesthetic and functional outcomes were achieved for all patients.

Conclusion: The new microbial cellulose dressing, Epiprotect ${ }^{\circledR}$, appears to be a patient-friendly dressing that is easy to apply and ensures optimum conditions for the treatment of superficial and mid dermal facial burns. It could also be used after facial dermabrasion, facial skin excoriation, laser treatments etc. The microbial cellulose with its nanostructure like collagen, adheres properly, works as a biological anchoring on the proteins of soft tissues and reaches the re-epithelization processes, by our clinical experiences, faster as hydrocolloid gels.

P121 A naturally occurring prospective study on skin repair after second degree skin burn injury in four family members

\section{März, PM Vogt}

\section{Medical School Hannover, HANNOVER, Germany}

Objectives: Intermediate second-degree skin burn injuries are a challenge for the therapists who have to handle them either with conservative and operative methods. Therapy options extend from stimulated spontaneous healing up to aggressive surgical debridement. In this study the outcome of similar second-degree skin burn injury is shown at the example of four family-members after a boat explosion in 2017.

Methods: Clinical data of the four family-members which were treated at our burn center in 2017 were used. The areas of burned skin $\left(\mathrm{IIa}^{\circ}-\mathrm{IIb}^{\circ}\right.$ ) extended from $14 \%$ to $38 \%$ TBSA.

Results: All wounds were covered with hydrogel and fatty gauze after an initial aseptic debridement.

Wounds of the girl (18\% TBSA) were secondly grafted with allogeneic skin. Hyperpigmented hot spots and instable skin texture were detected after two years.

Burned areas of the boy (20\% TBSA) were covered with temporary synthetic skin after debridement. All areas regenerated well with a moderate hyperpigmentation.

$14 \%$ TBSA of the mother were covered by meshed allogeneic skin. Today a hyperpigmentation and an instability of the skin is still visible.

Wounds of the father (38\% TBSA) was treated by allogeneic skin, keratinocytes suspension, temporary synthetic skin and autologous meshed skin. Today a hyperpigmentation and some instable areas, edema and scars in the lower leg persist.

Conclusion: The excellent results of this patient cohort suffering from the same accident and identical exposure to the explosive mechanism, support the importance of a stage-related therapy of the skin burn injury. In our view an initial debridement followed by early coverage is the key to early reconstitution of the epidermal barrier.

For second-degree burn injuries the treatment with temporary synthetic skin, temporary allogeneic skin grafts and the final treatment with autologous meshed skin or a cell suspension is the best option for excellent clinical and aesthetic results.

P122 The Use of Medihoney Wound Gel following Major Burn Injury

N Lee, N Martin, D Barnes

St Andrews Burns Unit, CHELMSFORD, United Kingdom 
Objectives: A retrospective review of eleven burns patients having topical Medihoney wound Gel applied to wound beds at different stages of resurfacing.

Active Leptospermum honey (ALH) has been reported to have salutary effects in the management in acute and chronic wounds. However, the use of ALH for the management of larger burn injuries with multiple microbiology has been sparsely reported. Medihoney Wound Gel is a topical preparation of Manuka honey reported to have antimicrobial properties that provide a barrier to wound pathogens maintaining moist and slightly acidic wound environment, conducive to wound healing.

Methods: Inclusion criteria-Adult burn patientsITU level supportNot responding to standard treatment, Burn percentage area $>15 \%$ TBSAShowing signs of wound infection \pm systemic infection/sepsis.

Patients were bathed with antimicrobial soap and wounds dressed with paraffin tulle gauze impregnated with MediHoney Wound Gel and secured with gauze and gamgee repeated every other day.

Results: All Patients were confirmed to have gram negative or gram positive infection from wound swabs + systemic infections. $90.9 \%$ showed improvement of systemic infection and wound bed overgranulation within $5-14$ days. 9.1\% did not see improvement due to early removal of honey from the wound bed. $27.2 \%$ reported a higher level of pain with $18.1 \%$ requiring removal of the honey dressings at different stages of treatment. Secondary outcome noted $27.2 \%$ of patients were only receiving parenteral feed, due to gut failure, compared to $72.7 \%$ on enteral feed all of which showed similar wound healing rates with the use of Medihoney.

Conclusion: Within all cases Medihoney was not the only change to care as patients required multiple and dynamic end organ support for septic with multiple changes to medication, antibiotics and level of care i.e. hemofiltration. However Medihoney has shown encouraging results with all participants.

P123 The Use of Hydrogen Peroxide in the Treatment of Infected Burn Wounds: A Literature Review and Questionnaire of Current Clinical Practice in the United Kingdom

TH Mihailidis ${ }^{1}$, PL Patenall ${ }^{2}$, KC Coy ${ }^{3}$, AY Young ${ }^{1}$

'University of Bristol, BRISTOL, United Kingdom

${ }^{2}$ University of Bath, BATH, United Kingdom

${ }^{3}$ University Hospitals Bristol NHS Foundation Trust, BRISTOL, United Kingdom

Objectives: Current treatment of infected burn wounds is with antibiotics and/or debridement, however bacterial resistance remains a significant problem. The aim of this study is to investigate the use of hydrogen peroxide $\left(\mathrm{H}_{2} \mathrm{O}_{2}\right)$ in the treatment of infected burn wounds as an alternative to antibiotics.

Methods: We sent an 11-question survey on the use of $\mathrm{H}_{2} \mathrm{O}_{2}$ in the treatment of infected burn wounds to all burn services in the United Kingdom using a list obtained from the British Burns Association. Additionally, we performed a literature search on the clinical use of $\mathrm{H}_{2} \mathrm{O}_{2}$ in the treatment of infected burn wounds.

Results: A $72.7 \%$ response rate was achieved. Of these, $75 \%$ do not currently use $\mathrm{H}_{2} \mathrm{O}_{2}$. Of the $25 \%$ which do, there is no established protocol on its use. The most commonly given reasons for not using $\mathrm{H}_{2} \mathrm{O}_{2}$ were a lack of published evidence, the demonstration of superior antiseptics or a fear of fatal sideeffects.

The literature search generated 1168 papers, with only one addressing the research question. This RCT demonstrated that soaking with $2 \% \mathrm{H}_{2} \mathrm{O}_{2}$ prior to grafting improved graft take rate in infected burn wounds compared with grafts treated with saline prior to grafting.

Conclusion: Whilst one RCT shows $\mathrm{H}_{2} \mathrm{O}_{2}$ to be an effective burn wound disinfectant, more largescale research in this field is required to determine whether $\mathrm{H}_{2} \mathrm{O}_{2}$ may offer a solution to antibiotic resistance in infected burn wounds.

Picture 1: https://www.eventure-online.com/parthen-uploads/89/9EBA/add_1_539626_23008c181c75-4e85-9dc4-9ece2857d259.png 
P124 A case series examining the use of EpiProtect on patients who have sustained partial thickness burn injuries.

\section{Presley-Hague}

University Hospitals Birmingham, BIRMINGHAM, United Kingdom

Objectives: The epidermis is almost always destroyed when a partial thickness burn injury is sustained. Wound healing can often be complex with frequent dressing changes that can be extremely painful and traumatic. EpiProtect is a single application dressing that has been engineered to act as a synthetic epithelium and has not been previously used at the Queen Elizabeth Hospital Burns centre. A case review was undertaken in order to identify the indications, contra-indications and benefits of its use.

Methods: This is a small single centre retrospective and prospective case series including a minimum of 5 cases. Suitable patients will be identified by senior medical/nursing staff from the burns centre. A review of medical documentation and evaluations provided by Regen medical will be undertaken for each patient. A literature review will also be undertaken to establish any previous use of the product.

Results: Initial retrospective 3 case review examined medical/nursing notes and evaluations. The evaluations reported a reduction in pain for patients and ease and speed of use for staff. The wound healing was described as clinically acceptable for 2 cases but the third was lost to follow up. The initial findings mirror results previously found with the product in literature. The retrospective review has identified a criterion for future patient selection within the centre with a minimum of 2 patients to be reviewed over a 6-month period. These will then be combined to complete the case series review.

Conclusion: Initial results replicate findings from previous studies and demonstrate that Epiprotect has a range of benefits for both patients and staff and its use will be continued within the centre. As a single centre study further research is required to replicate these findings on a wider patient group and consolidate the criteria for its use. Support from Regen Medical producers of Epiprotect is declared.

P125 Development and clinical application of a new foam dressing for negative pressure wound therapy

\section{IK Chai}

Burn institution of PLA, BEIJING, China

Objectives: At present, the widely use of negative pressure wound therapy (NPWT) has achieved remarkable therapeutic effects. However, some defects were found during its clinical use. Because the foam dressing is in the form of a block and inflexible, when it is used for large wounds such as entire limbs or special parts of the body such as hands, it is time-consuming and laborious, et al which affects its use and treatment effects. In this study, a new foam dressing for entire limbs and hands was introduced.

Methods: The new foam dressing with was thinner and much longer than the previous foam dressing and had a bandage-like appearance. It can be wrapped around the entire limbs and hands like a bandage. The drape for closing the limb wounds was much larger than the previous one. One or two pieces can seal the entire limbs (usually one piece for upper limb and two pieces for lower limb). A nonperforated drainage tube was used.

Results: When the new dressing was applied to the entire lower limbs, one staff could complete the operation within 10 minutes, while the previous foam dressing required 2-3 staffs to complete at least half an hour. Because of the reduction of the number of drainage tubes and the reduction of film splicing, the incidence of air leakage was reduced, and the wound sealing effect was better. The new dressing had lower pressure on limb than the conventional dressing and would not cause ischemia of the extremities. Clinical application showed that both the new dressing and the previous foam dressing had good treatment effects on the entire limb wounds and wounds of hands.

Conclusion: The new foam dressing for NPWT is safe, convenient to operate, time-saving, laborsaving, and has good sealing effect for entire limbs and hands. 
P126 Decellularized Human Dermal Matrices for the Treatment of Burn Injury

T. Rose, J-P Draye, M Boone, G Verween, G Verbeken, S De Soir, P De Corte, B Pascual, S Jennes, J-P Pirnay

Queen Astrid Military Hospital, BRUSSELS, Belgium

Objectives: Recently, we have developed a novel Decellularized Human Dermal Matrix (DHDM), having both 3D-structure and composition well preserved. In order to decrease the need for autografting in patients with extensive deep burns, we plan to use this DHDM, together with autologous cells. The main objective of this preliminary work is the evaluation of the integration of the DHDM in burn wounds and its ability to receive skin autografts.

Methods: Cryopreserved allogeneic skin (about $0.4 \mathrm{~mm}$ thick) was obtained from post mortem human donors and was used to prepare DHDMs. A two-step decellularization method, involving a hypertonic treatment followed by a detergent treatment, was used to prepare the DHDMs. After detergent removal, the DHDMs were cryopreserved. Bacteriological/mycological testing, histological evaluation, MTT viability testing and High-Definition Optical Coherence Tomography imaging were used for quality control. The DHDMs were applied to burn wounds and were maintained in place by stitches. Wounds were dressed with transparent polyamide dressing and silver nanocrystalline dressings. Visual evaluation, with photographic records, and histological evaluation of the DHDM integration were performed.

Results: It was observed that burn wounds treated with DHDM were not infected. After about 3 weeks the DHDMs were integrated into the burn wounds and wounds were ready to receive skin autografts.

Conclusion: Conclusively, the DHDMs integration in burn wounds can be achieved. In the next step the use of autologous cells together with DHDM should improve revascularisation, shorten the integration time and reduce the need for autografting in patients with extensive deep burns.

P127 Antimicrobial resistance in burn wound care - evidence to support a role for nanocrystalline silver to tackle this increasing global problem.

\section{E J Woodmansey ${ }^{1}, C$ D Roberts ${ }^{2}$}

${ }^{1}$ Smith\&Nephew, HULL, United Kingdom

${ }^{2}$ Clinical Resolutions, HULL, United Kingdom

Objectives: The issue of antimicrobial resistance (AMR) is an ever increasing global concern. Wound care is no exception; in burn centres the selective pressure from high antibiotic use, ineffective antibiotic dose at site of injury due to altered drug pharmacokinetics and pharmacodynamics and longer hospitalization and treatment regimens all may influence the risk of resistance development.

Antibiotic resistance and stewardship (AMS) have been the priority for most strategic interventions so far, however, in wound care other alternative or supplementary strategies using antiseptics should be considered. Local antiseptics such as silver can provide effective cidal activity across a broad range of wound pathogens including antibiotic resistant organisms, assuming they are used in the correct dose for an appropriate time period. The aim of this study was to understand the role of nanocrystalline silver barrier dressings as part of a strategy to tackle antimicrobial resistance in burns.

Methods: A search of published evidence that supports the position of effective silver barrier dressings containing nanocrystalline silver within an AMR and AMS strategy for wound care literature was performed. The evidence supporting the role of this silver barrier dressing in burn wounds was subsequently summarised in the context of infection control, appropriate antimicrobial use and reduced antibiotic use.

Results: An increasing body of evidence is available to support nanocrystalline silver use to minimise transmission of antibiotic resistant organisms as part of infection control procedures and in addition, through appropriate early use and stewardship on local wound infections, to minimise the need for systemic antibiotic therapy in surgical wounds and burns.

Conclusion: Engagement, alignment and collaboration between wound care professionals and wider related teams and governments on antimicrobial stewardship, and the potential role of 
antiseptics within this will help to progress further evidence for such interventions in the fight against antimicrobial resistant infections in wound care.

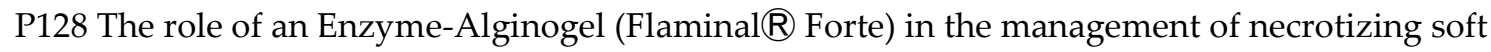
tissue infections (NSTI).

\section{K Decock}

UZ Leuven, HERESTRAAT 49, Belgium

Objectives: The Burn Centre/ICU of the University Hospitals Leuven has seen a significant rise in patients referred with necrotising soft tissue infections from 0-3 to 15-25 per year over the last 15 years. Hospital mortality is $10 \%$. The multi-disciplinary therapy includes prompt and aggressive surgical excision, triple antibiotics and for some patients prolonged mechanical ventilation, haemodynamic support (including ECMO), immune globulins and haemodialysis.

The aim of this case report is to present our centres' wound treatment strategy for NSTI, standardised over the last decade.

A 52 year old male patient was admitted with an NSTI affecting the right scapular region. After initial excision, the wound had a TBSA of $9 \%$, extending from the right upper arm over the axilla to his back and reaching into the muscle.

Methods: The wound treatment entails 3 major aspects: hydrotherapy, application of an EnzymeAlginogel (Flaminal ${ }^{\circledR}$ Forte) and negative pressure wound therapy (VAC).

Initially the patient received daily hydrotherapy therapy; rinsing and cleaning the wound with a Hibitane solution $0,05 \%$ on a shower trolley followed by application of the Enzyme-Alginogel. Subsequently VAC therapy promoted granulation in the wound and wound cavity's, preparing the wound for skin grafting. VAC therapy was interrupted every 4 to 5 days by 2 days of Enzyme-Alginogel application. All wound care procedures included hydrotherapy. A combined approach aimed at optimizing neovascularisation, granulation and mechanical reduction of bacterial load and debris.

Results: This complex and extended wound could be grafted after only 5 Weeks of multimodal wound care.

Conclusion: The combination of hydrotherapy, Flaminal ${ }^{\circledR}$ forte and negative pressure wound therapy enables wound preparation for skin grafting even for the deep and complex lesions generated by aggressive resection of NSTI.

\section{P129 HONEY FOR THE TREATMENT OF FACIAL BURNS}

ISA SAEZ AVILES, IFF FUENTES FERNANDEZ, JGJ GARCÍA JUARRAN, ASB Sánchez Balado, MUO UMAÑA ORDOÑEZ, CRA RECALDE ANDRES, ANT NOVO TORRES, ELB LORDA BARRAGUER

ALICANTE GENERAL HOSPITAL, ALICANTE, Spain

Objectives: The management of 2nd degree facial burns has been the subject of study for decades; In this field, Medihoney ${ }^{\circledR}$, thanks to its antibacterial properties and promoters of the healing of honey, is postulated as a promising treatment alternative, recently evaluated with good clinical results.

Methods: Manuka honey dressing has long been available as a non-antibiotic treatment in the management of burn wounds.

Results: We treat patients with facial burns of various etiologies (flame, electric flash, deflagration and scald). No treated patient presented clinically manifest infection or required surgical debridement, autograft coverage or sequelae surgery.

Conclusion: Our preliminary experience indicates that the Medihoney ${ }^{\circledR}$ application could be very useful in superficial facial burns.

P130 Flaminal in The Management of Bilateral Knee Cement Burns

B Noble

The Newcastle upon Tyne Hospitals NHS Foundation Trust, NEWCASTLE - UPON - TYNE, United Kingdom 
Objectives: Contact with wet cement can cause several types of skin reactions from mild irritation to full thickness chemical burns (liquefaction necrosis) due to the alkalinity of cement ( $\mathrm{pH} 12)^{1}$ Alkali combines with cutaneous lipids to create 'soap' and thereby continues dissolving the skin until it is neutralised. ${ }^{2}$

The objective of this case study is to discuss the use of Flaminal in the management of bilateral knee cement burns on a 37-year-old male, who sustained the burns at work whilst kneeling in cement for a prolonged period of time. The onset of injury with cement is insidious and John was initially unaware of the problem until several hours later.

Methods: On admission to the burn centre, John's wounds were 'horseshoe' shaped around each patella (possibly caused by increased alkalinity in solution in surface water on cement), necrotic, dry and discoloured deep red/black. The initial aims of treatment were to hydrate and debride the necrotic tissue, reduce bioburden, manage exudate and neutralise the $\mathrm{pH}$. Flaminal hydro, an alginate gel with an antimicrobial enzyme complex was selected as the primary dressing covered with a non-adherent contact layer, burn gauze and crepe bandages. Dressings were changed daily for the first week, then alternate days as the necrotic tissue started to separate. A Laser Doppler Imaging (LDI) scan was performed at $48 \mathrm{hrs}$ to establish blood flow into the burn.

Results: John's wounds were debriding well after two weeks. The focus of care then shifted to prevention of infection and pain management. John found the dressing to be soothing and comfortable.

His wounds eventually required skin grafting but to a much smaller area than suggested by LDI and clinical assessment.

Conclusion: This case study demonstrates the effectiveness of an enzyme alginogel in assisting autolytic debridement, control of bioburden plus moisture balance in the management of chemical burns. ${ }^{3}$

P131 Influence of Secondary Dressings on the Effect of Epicite Hydro - Results of an Animal Study

\section{A.-C. Tuca}

Medical University of Graz, GRAZ, Austria

Objectives: A balanced moist wound environment can increase and stimulate cell growth and wound healing. We tested the effects of different secondary wound dressings and their evaporation of water when used with Epicite Hydro ${ }^{\circledR}$ in vitro and in vivo in a porcine donor site model. The aim of this study was to evaluate how the different rates of evaporation affect wound healing.

Methods: Epicite Hydro ${ }^{\circledR}$ is a hydroactive wound dressing made from pure biotechnologically derived cellulose Epicite Hydro ${ }^{\circledR}$ was tested in vitro, either alone or in combination with secondary wound dressings used in clinical routine such as cotton gauze, Jelonet ${ }^{\circ}$, Aqacel ${ }^{\circledR}$ Extra and Opsite ${ }^{\circledR}$ Flexifix. Furthermore, in a porcine donor site model, we analyzed the effect on wound healing in vivo.

Results: The in vitro experiments showed different rates of water evaporation from Epicite Hydro ${ }^{\circledR}$ when using different secondary dressings. The results from the animal study showed that Epicite Hydro ${ }^{\circledR}$ in combination with cotton gauze or Aquacel ${ }^{\circledR}$ Extra had comparable high rates of reepithelialization.

Conclusion: We were able to show that the moisture of the wound environment and evaporation from the wound can be influenced by using Epicite Hydro $\AA^{\text {in }}$ combination with different secondary dressings. This makes it possible to positively influence cell growth and thus accelerate wound healing.

P132 Severe burn in child with hypoplastic left heart syndrome.

A Ziola ${ }^{1}, \underline{\text { AM Bulandra }}{ }^{2}$, T Koszutski ${ }^{2}$

${ }^{1}$ Uppersilesian Child Health Center, KATOWICE, Poland

${ }^{2}$ Medical University of Silesia, KATOWICE, Poland

Objectives: Hypoplastic left heart syndrome (HLHS) is a complex defect involving the left ventricle hypoplasia, stenosis or occlusion of mitral and aortic valve, as well as hypoplasia of the ascending aorta and the arch. Children with this syndrome present various symptoms of circulatory insufficiency. This makes the treatment of burn shock particularly difficult and impairs wound healing.

Methods: A case of 3-years old girl with HLHS is presented. She suffered extensive second and 
third degree burns of the face, thorax and abdomen - at least 30\% TBSA.

Results: When admitted to the hospital, the girl was in a burn shock, which required treatment in the intensive care unit. Due to circulatory disorder manifested by reduced skin flow and blood saturation, spontaneous healing of burn wounds was significantly delayed. Furthermore, in the coherent opinion of anaesthesiologists, cardiologists and cardiac surgeons, the patient did not qualify for surgical treatment under long-term general anesthesia. After initial stabilization of the cardiac state of the child, the multi-stage treatment was performed. During several short-term shallow anesthesia necrotic tissues were removed and split-thickness skin grafts were done. Graft and donor sites healing was considerably prolonged but uncomplicated. After 8 weeks of treatment, the girl was discharged home.

Conclusion: Effective treatment of severe burned child with a complex heart defect is very difficult. It requires the involvement of many specialists and the use of unconventional treatment methods.

P133 Management following Nexobrid? rapid enzymatic debridement of burn wounds, our experience with the use of ReCell? activated keratinocyte suspension.

T K Randawa, C Harris, J Stallard, PM Muthayya, A Phipps, U Anwar

Pinderfields Regional Burns centre, WAKEFIELD, United Kingdom

Objectives: We have been using Nexobrid for the past three years. We present our learning from this evolution and describe our current protocol of combination allograft with activated keratinocyte suspension spray.

Methods: For all patients who underwent Nexobrid debridement of their burn injury\&application of ReCellâ activated keratinocyte suspension, Patient Demographics, mechanism, \% TBSA of the burn injury were recorded. Outcome data, including number of trips to theatre, time to healing, and\%TBSA requiring autograft were noted

Results:

We identified a total of 10 patients who were managed with ReCellâ activated keratinocyte spray following enzymatic debridement, both in isolation and in combination with xenograft or allograft. ReCell was found to improve pain control following Nexobrid debridement.In combination with allograft,the number of trips to theatre,time to healing and\%TBSA requiring autograft were all reduced.

Conclusion: Based on our success strategy and experiencesour centre increased their scope of the use of Nexobrida to include resuscitation level injuries (defined as TBSA 15\% or greater $18-65$ years, $10 \%$ or greater over 65 years). This then evolved to combination ReCellâand allograft in larger injuries and has led to excellent results in our patient population.

In our case series the use of ReCellâfollowing Nexobridâ debridement has improved pain management and when used in combination with allograft has facilitated a reduction in trips to theatre, $\%$ TBSA requiring autograft and time to healing.

P134 Designing an algorithm for the use of Suprathel $\mathbb{R}$ following enzymatic debridement with Nexobrid $\mathbb{R}$ in burn injuries in hands

BP Dos Santos Marcano, J Serracanta Domenech, J Aguilera Saez, JM Collado Delfa, A Monte Soldado, G Antelo Coroas

Vall d'Hebron University Hospital, BARCELONA, Spain

Objectives: Describe our experience using Suprathel ${ }^{\circledR}$ after enzymatic debridement of burn injuries located in hands

Methods: Patients presenting with second degree intermediate burns located in their hands whether inpatient or outpatient were treated with enzymatic debridement with Nexobrid®

Next day assessment of the wound bed resulted in some patients moving from intermediate to predominantly deep or superficial second degree.

Patients with predominantly superficial or intermediate wound beds after debridement were treated wtih Suprathel ${ }^{\circledR}$

Results: Patients treated sequentially with Nexobrid ${ }^{\circledR}$ and then Suprathel ${ }^{\circledR}$ showed as per the literature and our experience a similar delayed healing time as is expected with Nexobrid® alone. 
Nonetheless patients who underwent adjunctive treatment with Suprathel ${ }^{\circledR}$ required less surgical debridement and grafting

Conclusion: Debridement and grafting extend and application is a challenging decision every burn unit surgeon must face. Our understanding of what constitutes a burn that should ideally be treated in the OR has been somewhat shifted with the advent of ancillary diagnostic and therapeutic procedures such as Laser Doppler imaging scan and enzymatic debridement, the latter has somewhat lifted the veil from the preconceived pattern experience has taught us to recognize and assess an "uncontaminated" wound bed were the depth of dermal involvement is more readily apparent.

This process of selective debridement that allows for a reconsideration of our diagnostic acumen also leaves the wound bed more vulnerable to desiccation and might even remove some of the extracellular cues that trigger proper wound healing, and it is for this reason that Suprathel ${ }^{\circledR}$ complements the process of enzymatic debridement by providing a flexible porous membrane that mimics the protective properties of healthy epithelium with a high capacity for water absorption creating an ideal environment for wound healing

P135 Surgical versus conservative treatment in pediatric thermal burns

DM Enescu, S Stoicescu, D Ionita, R Tatar, M Tomita

'Grigore Alexandrescu' Hospital, BUCHAREST, Romania

Objectives: Our burn center has an experience of over 30 years and over 17000 pediatric burns treated. Choosing between surgical and conservative treatment can be difficult in many situations. The experience of our center emphasizes some elements that could facilitate the decision and improve the outcome of the patient. Our goal is a fast rehabilitation with minimal sequelae.

Methods: Admitted patients were evaluated over the before mentioned extended period of time. The choice of treatment, hospital stay, scars properties were recorded among other particular details.

Results: The decision to follow either of the two alternatives of treatment is taken according to many parameters such as the general state, patient's personal history, family history, age, burn surface, depth and severity. No decision can be made in advance; the experience of the center supports this. For each child a unique negotiation for treatment is undertaken.

There is no panacea among the topical used. We have a complex of topical ointments which we use according to many factors such as the local evolution or the general state of the patient.

The surgical treatment has to be adjusted according to another group of factors such as burn depth, extent, location.

Conclusion: For the best outcome of the patients it is highly useful reaching the optimal tinge of treatment between the surgical option which includes its specific risks for certain long term complications and adjusted local conservative treatment which may lead to similar results, in a similar time period for certain patients.

P136 The possibility of using «Burnshield» bandages in patients undergoing outpatient treatment.

V.S. Borisov, A.V. Sackov, M.Y. Kaplunova, K.V. Svetlov, M.E. Makarova

Burn Center of the N.V. Sklifosovsky Institute for Emergency Medicine, MOSCOW, Russia

Objectives: to evaluate the efficiency of "Burnshield" bandages with hydrogel in the local treatment of burn wounds in in ambulatory patients

Methods: "Burnshield" bandages represents a flexible foamy polyurethane base impregnated with "Burnshield" hydrogel composed of $96 \%$ water, 0,025\% ethanol, 1,03\% Melaleuca alternofolia (tea tree oil) and 1,5\% propylene glycol. The evaporation of his contact with burn wound results in cooling of the baked surface. Sick notes the cessation of pain. We observed 12 patients aged from 19 to 70 years: 8 men, 4 women with burns of I-II degree with a burn area 3\% TBSA, who received non-hospital treatment.

Results: "Burnshield" bandages well tolerated at all patients. Allergic reactions have been noted. The bandage very easily placed over the wounded surface. The patients could move independently or move wounded or tied parts of the body painlessly. Removing bandages passes easily and virtually painless. There were no signs of inflammation of the burn wound. 
Epithelialization of burn injuries was at the optimum time: I degree burns healed on average of 6,0 $\pm 1,3$ days $(p<0,05)$, burns II degree healed in of $9,0 \pm 2,1$ days $(p<0,05)$ since the injury.

Conclusion: "Burnshield" bandages is an very effective for local treatment of patients with burns in patients undergoing outpatient treatment.

P137 Activated platelet-rich plasma therapy for subacute second degree burns: a case report

K Karina ${ }^{1}$, APB Sarena ${ }^{2}$, T Toar ${ }^{2}$

${ }^{1}$ Hayandra Clinic, JAKARTA, Indonesia

${ }^{2}$ Faculty of Medicine Universitas Indonesia, JAKARTA, Indonesia

Objectives: Intensed pain followed by bad scar result is the most common problem in current burn wound standard treatment. Thus, subacute wound healing which prolong wound healing process and increase pain because of poor cytokines activities and growth factors will further the burden of burn patients. In this study, we want to evaluate the effect of enhancing the activities of growth factors by adding activated platelet-rich plasma (PRP) treatment in subacute burns regarding the wound heal process, pain reduction, and scarring result.

Methods: This study report a PRP-treatment on 40-year old female with a subacute second-degree burn on her left arm. The patient came 10 days post-injury with 3\% TBSA deep partial thickness burns, a sign of secondary infection and a flexion contracture after being treated previously in another hospital. The activated PRP treatment were given via intravenous and intralesion after debridement procedure. Oral antibiotic and analgetic were given on this patient too. The wounds was cleansed conventionally with a wound gel and no skin graft procedures applied to this patient. The follow up was taken every week in 4 consecutive week after the PRP-treatment.

Results: The patient reported a reduction of pain after receiving PRP treatment. We found $100 \%$ epithelization within 3 days after the treatment. Left arm flexion was completely gone with no signs of infection. After 1 month, no hypertrophic scar, pliability, or itchiness were reported.

Conclusion: Treatment using activated PRP for subacute burn wounds appeared to be effective. Activated PRP could improve quality of life with the reduction of pain, shorten recovery period and better scar appearance. As a minimal invasive treatment, it would be a great consideration to replace skin graft as the standard burn therapy.

P138 Split thickness skin grafts (STSGs) reflect differences in early composition of extracellular matrix on wound bed year later in proteomic analysis - a comparative study

HM Lagus $^{1}$, M Klaas ${ }^{2}$, S Juteau ${ }^{3}$, O Elomaa ${ }^{4}$, J Kere ${ }^{5}$, J Vuola ${ }^{6}$, V Jaks ${ }^{7}$, E Kankuri ${ }^{8}$

${ }^{1}$ Helsinki Burn Centre and Wound healing centre, Helsinki University Hospital, HELSINKI, Finland

2Institute of Molecular and Cell Biology, University of Tartu, TARTU, Estonia

${ }^{3}$ Department of Pathology, Haartman Institute, University of Helsinki and HUSLAB,, HELSINKI, Finland

${ }^{4}$ Folkhälsan Research Center, HELSINKI, Finland

${ }^{5}$ Karolinska Institute, Department of Biosciences and Nutrition Huddinge, STOCKHOLM, Sweden

${ }^{6}$ Helsinki Burn Centre, Department of Plastic Surgery, Helsinki University Hospita, HELSINKI, Finland

${ }^{7}$ Department of Cell Biology, Institute of Molecular and Cell Biology, University, TARTU, Estonia

${ }^{8}$ Faculty of Medicine, Department of Pharmacology, University of Helsinki, HELSINKI, Finland

Objectives: Dermal substitutes provide scaffold for autologous cells to reconstruct dermis. The micromolecular environment is different from granulation tissue and may affect the signaling cues during wound healing and final outcome. We compared the effect of three different wound bed compositions on STSGs protein content one year after operation.

Methods: Test area of 10x15 cm of a large deep burn after excision of four patients was divided into three $10 \times 5 \mathrm{~cm}$ test sections: one covered with STSG as a control group, one with artificial dermal substitute Integra and one with cellulose sponge as a temporary cover inducing granulation tissue. 
After two weeks the test sections treated with dermal substitute and cellulose sponge were also transplanted with STSGs. After one year biopsy samples were taken from each test section and analyzed with site-targeted proteomics and validated using immunohistochemistry to detect differences between treatments.

Results: Out of the 481 proteins identified $12 \%$ were significantly differentially expressed $(p<0.05)$ between the different test sections; 30 proteins of epidermis and 24 proteins of dermis. The biggest difference between epidermal proteins was found in axonemal heavy chain dynein DNAH10 with 34fold difference between the control group and the induced granulation tissue after cellulose sponge treatment. In dermis the four biggest differences were detected in expression of keratins KRT1, KRT6C, KRT16 and KRT77 likely from skin appendages; their expression was higher in induced granulation test section compared to other test sections.

Conclusion: Different dermal matrix compositions during early wound healing seem to have long term or permanent effects on split thickness skin grafts. The significance of DNAH10 for wound healing and epidermal maintenance requires further investigations.

P139 Enzymatic debridement of genital burns as alternative to common approaches

\section{MR Ribitsch}

Cologne-Merheim Medical Center, COLOGNE, Germany

Objectives: Usually deep burn injuries are treated with early debridement. In contrast to this, many burn surgeons prefer a wait-and-see behavior when it comes to deep genital burns. A successful outcome is particularly important for rare genital burns, though especially here only few information and treatment guidelines can be found. In order to promote spontaneous healing, the debridement should be as modest as possible, but sufficient to facilitate spontaneous healing.

A new therapeutic approach is the tissue-sparing enzymatic debridement of genital burn wounds based on bromelain, which we compared to current therapies.

Methods: First a literature review on the treatment of genital burns was performed on PubMed between 1990 and 2016. Afterwards all patients were evaluated who were treated for genital and perineal burns between 1995 and 2016 in our clinic.

Results: As reported in most cases found in the literature, our 149 patients with genital and perineal burns were treated conservatively in a wait-and-see behavior.

If necrosis of the skin developed in the course of the treatment, they were debrided surgically and covered with a split skin graft.

All three patients with genital burns treated with enzymatic debridement healed spontaneously without the need of skin grafting and with good functional and aesthetic outcome.

Conclusion: Compared to surgical debridement of genital burns, enzymatic debridement helps to preserve vital tissue in this sensitive region which can reduce the need of skin grafting and can help achieve good aesthetic and functional results. Therefore enzymatic debridement is an interesting alternative for these burn injuries.

\section{P140 First Experitnce with Gidrogel ${ }^{*}$ on the Treatment Burn Wound in Acute Period}

\section{V.S. Borisov, A.V. Sachkov, O.V. Troshina, M.Y. Kaplunova, K.V. Svetlov}

Burn Center of the N.V. Sklifosovsky Institute for Emergency Medicine, MOSCOW, Russia

Objectives: to evaluate the efficiency of hydrogel ${ }^{*}$ in the treatment of burn wounds in first 24 hours after burns.

Methods: the study included 12 patients with burns of I-II degree with a burn area 5-8\% TBSA who asked for help at the clinic of the burn center up to 30 minutes after the accident, and to whom were not applied any significant treatment of wounds. 7 patients were included in group I with "BURNSHIELD" hydrogel, composed of $96 \%$ water, $0.025 \%$ ethanol, $1.03 \%$ Melaleuca alternofolia (tea tree oil) and $1.5 \%$ propylene glycol. In the other group of 5 patients, where the wounds were treated in the traditional manner. We evaluated analgesic and healing effects

Results: In group I epithelialization of burn wounds occurred in terms of $8,0 \pm 1,3$ days $(p<0,05)$. Because of its structure, the hydrogel evaporated and caused cooling and anesthesia of the ocular 
wound: pain was assessed $2.5 \pm 1.1$ points on a visual analogue scale (VAS). In no case generalization of the inflammatory process was observed. In the group II the wounds were epithelialization in $13+5,6$ days $(p<0,05)$. Dressing changes for the patients of the group II were painful, level of pain was $6.1 \pm 0.5$ points (VAS). In 50\% of cases in the group II the presence of copious purulent discharge was noted. Before starting treatment all patients has $68 \%$ allocated to monoculture. After treatment in group I the wounds were clean, in the group II weren't.

Conclusion: hydrogel ${ }^{*}$ is an very effective for local treatment of patients with burns.

"BURNSHIELD" hydrogel

P141 Some Therapeutic Consideration in Our Experience Treating Severe Burned Patients

G NARAD, P TASKOV, E.C HORDOVAN, V NARAD, M NEMES, M SARANDAN, D.M CORODATI, G NODITI

Emergency County Hospital „Pius Branzeu,,, Timisoara, Romania, TIMISOARA, Romania

Objectives: To summarize the experience of treatment in extremely severe burn patients.

Methods: The clinical data and treatment of 2 severe burn patients with TBSA $>80 \%$

There were 1 male and 1 female, aging 27 and 41 years, with 85\% and 82\% (TBSA) and fullthickness of $\sim 65 \%$, respectively $35 \%$ TBSA. The both patients were admited with severe shock, inhalation injury, and blast injury after 10 hours and 30 hours. We have encounted some major deficiencies related to skin bank because both pacients died after 21 days, respectively 60 days in ICU with MOF and $20 \%$ of covering, respectively $35 \%$ for de female pacient.

Our unit has quite rich experience in enzymatic debridment treatment with satisfactory results.

Escharectomy was necesary for both patients and enzimathic debridement were performed until 7 days after injury for several series.

Covering with allogeneic skin and autoskin were performed 7 to 30 days after injury.

Results: AKI occurred in both patients and their renal function was guided by thermodilution catheter (Picco) and application of renal support.

Pulmonary complications, such as late ventilator-associated bronchopneumonia, occurred in both pacients.

The wounds were basically healed in both patients in $\sim 7-45$ days by 4 or 5 times of operation (allografts, autografts) and spontaneus epithelization.

Conclusion: Enzymatic debridment is very usefull in cases with extensive burns, especially in partial thickness burns what avoids the decompression incisions and escharectomy in critical pacients and for fast epithelization.

Early closing wound as soon as possible is the key to successful treatment and the pacient survival.

It's imperious necessary a national skin bank or a collaboration with a foreign skin bank for early covering the wounds.

Treatment strategies in team multidisciplinary cooperation by department of burns are the bases to successful treatment.

P141 Treatment of partial thickness hand burn injuries in children with combination of silver foam dressing and zinc-hyaluronic gel.

IG Jozsa, CSA Csenkey, JZS Juhasz

Department of Pediatrics, Surgical Unit, PÉCS, Hungary

Objectives: Burns is a common type of traumatic injury in childhood. Nowadays, several wound dressings are available to treat the second-degree hand burns conservatively.

Methods: At the authors' institute, 37 children were treated conservatively with a special dressing at first intervention containing Aquacel $\mathrm{Ag}$ foam and $\mathrm{Zn}$-hyaluronic gel to determine their effectiveness on partial thickness hand burns. The dressing was checked on the second day, and removed on the sixth or seventh day (unless it had spontaneously separated).

Results: None of the 37 children treated with this dressing were diagnosed with wound infection. The authors observed the epithelialization of the burned areas on the $6-7^{\text {th }}$ day after primary conservative treatment. The dressing efficiently promotes epithelialization in all cases. Further 
advantage of Zn-hyaluronic gel is to enhance cell regeneration and inhibits dressing fixation into the wound.

Conclusion: Based on the authors' experience, with this special combination of wound dressing, a gentle, child-friendly, cost-effective treatment and excellent wound healing observed with favourable cosmetic results.

P143 Pressure necrosis - a burning issue? case presentation and review of literature.

B Bednarz, M Thakkar, I Mackie, E Estela, S Sen

North Bristol NHS Trust, BRISTOL, United Kingdom

Objectives: To the majority of health professionals, burns present a challenging, potentially distracting, diagnosis. Because of their perceived complexity, they often eclipse other medical problems which can be life threatening. Pressure necrosis may present with full thickness skin loss and in extreme cases expose underlying structures. In rare instances, such necrosis may mimic and be mistaken for full thickness burns. Such long lies may cause pressure necrosis of decubitus areas and compartment syndrome of vulnerable areas. Compartment syndrome, is a surgical emergency requiring prompt diagnosis and intervention. It may be missed in the context of a long lie after collapse. Such missed diagnoses, maybe detrimental to patients' outcomes. Herein we present two such cases .

Methods: We reviewed cases referred to our unit in the last four months, their clinical notes and medical photography. We have also performed literature search to find similar case reports.

Results: Two cases, with acute pressure necrosis from long lies had been mistaken for burn wounds, were referred to our burns unit in last four months. In one case a missed compartment syndrome resulted in a below elbow amputation. Images, with consent for publication, show a mode of injury causing pressure necrosis.

Conclusion: Pattern analysis and recognition are very important diagnostic tools in medicine. Detailed history taking and examination cannot be emphasised enough. Training both emergency departments and plastic surgeons in recognising long lie related injuries will decrease possible associated dangers such as missing a compartment syndrome.

P144 Silverlon - An alternative silver dressing for burns

IV Justine ${ }^{1}$, H Gerrish ${ }^{2}$, N Fox ${ }^{2}$, K Cranmer ${ }^{2}$, P Dziewulski²

${ }^{1}$ St Andrew's Unit for Burns \& Plastic Surgery, WATFORD, United Kingdom

${ }^{2}$ St Andrew's Unit for Burns and Plastic Surgery, CHELMSFORD, United Kingdom

Objectives: It is easy to get in the habit of using the same dressings, without testing the benefits of alternative products on the market. We review Silverlon, containing silver; $546 \mathrm{mg} / 100 \mathrm{~cm}^{2}$ vs AG Acticoat $\left(105 \mathrm{mg} / 100 \mathrm{~cm}^{2}\right)$.

Methods: We present 20 patient cases admitted through our Burns ITU where wounds were dressed with Silverlon $(n=10)$ compared against a control group of patients randomised to an alternative silver dressing (AG Acticoat, $\mathrm{n}=10$ ).

The evaluation included patients' medical history, nutritional status and burn wound parameters assessed by the nursing staff doing the dressing who completed an evaluation form, and infection was identified on wound swaps.

Results: The varied dressing sizes of Silverlon included a chest shape and 'gloved hand' dressing making it easier to dress wounds. In Silverlon-treated patients, we observed a lower infection rate (possibly due to slower release of silver ions, which have anti-bacterial action), a good healing rate and patients reported reduced pain, probably because of less-adherence of Silverlon to wounds.

Conclusion: The lessons from this small study help show that it is worth exploring new dressings to obtain the most efficient wound healing and to constantly improve medical care.

If one considers that Silverlon was actually less expensive than AG Acticoat, the evaluation resulted in good cost-effectiveness, as well las enhanced benefits to the patient.

P145 The use of Epiprotect $\mathbb{R}$ after enzimatic debridment to heal pediatric patients with burns: a case series. 


\section{G Delli santi}

Plastic surgery, ROMA, Italy

Objectives: The objective of this case series was to evaluate on pediatric patients with burns the use of a wound dressing based on the new material eiratex ${ }^{\circledR}$, composed of a biosynthetic cellulose network that resembles the nanostructure of collagen.

Methods: A total of $n=5$ pediatric patients (age $<8$ years) with burns were treated with the eiratex ${ }^{\circledR}$-based wound dressing after enzymatic debridement using NexobridTM.

The mean total body surface area (\%TBSA) affected by the burn was $5.6 \%$. Mean healing time was $31.2 \pm 3.5$ days since the occurrence of the burn.

Results: The results of this case series show the efficacy of the eiratex®-based wound dressing to heal the burn wounds of this study. Moreover, we show how the use of this wound dressing decreases the frequency of dressing changes, with a benefit for patients and a decreased cost for the healthcare system.

Conclusion: Burn injuries are a major cause of hospitalization and are associated with morbidity andmortality. Burns in children under the age of 16 represent a fourth of the burns occurring annually all over the world, with a peak under the age of 5 years. Pediatric burns represent an enormous socioeconomic problem for individuals and the health care system.

Standard of care of burn wounds consists of cleaning and debridement, followed by the use of different type of dressings and, when necessary, skin transplantation surgery. Most of the dressings used for burn wounds need to be changed with a certain frequency. The painful procedure of dressing changes in children with burns represent a big issue, causing anxiety and affecting even more the complex psychological status of those patients.

Picture 1: https://www.eventure-online.com/parthen-uploads/89/9EBA/add_544415_0058087f24e2-418d-b21e-b3ec1884eaed.JPG

Picture 2: https://www.eventure-online.com/parthen-uploads/89/9EBA/add_2_544415_0058087f24e2-418d-b21e-b3ec1884eaed.jpg

Picture 3: https://www.eventure-online.com/parthen-uploads/89/9EBA/add_3_544415_0058087f24e2-418d-b21e-b3ec1884eaed.JPG

\section{P146 Exudate Management On Burn Wounds with A New Superabsorbent Wound Dressing}

JR Rönningen ${ }^{1}$, AH Hindhede ${ }^{2}, \underline{\text { CH Hasselgren }}^{1}$

${ }^{1}$ Absorbest AB, KISA, Sweden

º̈rebro Kommun, ÖREBRO, Sweden

Objectives: To evaluate the exudate management of a new superabsorbent dressing on patients with exuding burn wounds.

Methods: Eight patients, aged 28-77 years, participated in this open case study. The patients were recruited and selected from a burn center because of their exuding wounds, ranging from superficial dermal to full thickness burns. Standard protocols were applied to patients included in the study. The time of participation in the study ranged between two and 12 days with an average length of seven days.

Results: All clinicians found the new superabsorbent wound dressing superior to standard treatment, i.e. fluffy gauze, in its ability to manage exudate and prevent maceration and hypothermia. Dressing changes in this study were generally due to the need for wound inspection and not because of leakage. In seven out of eight cases, the clinicians would recommend the new superabsorbent wound dressing for their local wound treatment program.

Conclusion: Results from this case series indicate that the new superabsorbent wound dressing is a suitable choice for exuding burn wounds because of its excellent capacity to manage exudate and prevent leakage, maceration and hypothermia, thus supporting patient recovery.

P147 Our Experiences with the Use of Hydrofiber Containing Combination Dressing for Donor Sites: A Pilot Study

I Juhasz ${ }^{1}$, I Erdei ${ }^{2}$, Z Peter $^{1}$, M Mate'1, GY Szoke 
1Burn Unit at University of Debrecen, DEBRECEN, Hungary

22University of Debrecen, Medical Faculty, Department of Anesthesiology and Inten, DEBRECEN, Hungary

Objectives: Foam dressings are widely used in treating various types of wounds. The addition of a contact hydrofiber layer created a combined dressing with improved moist handling ability with fewer tendencies for wound edge maceration. This results in acceleration of wound healing and better scar quality.

Methods: A prospective observational study was done involving 17 patients with donor sites after harvesting split thickness skin grafts (STSG). Gender distribution, initial wound size, time to healing, number of dressing changes, pain (VAS) and scar quality (VSS) were recorded.

Results: 11 female and 6 male patients with an average donor wound size of $105 \mathrm{~cm}^{2}(26-300)$ were enrolled. Complete epithelization took place in average 11 days (7-21), mean dressing changes required were 5 (2-10), 2 pts reported significant pain, while 11 pts had only minimal pain at dressing removal. Scar quality was excellent in 16 individuals; slight hypertrophy was noted at 1 .

Conclusion: Wound dressings with a combination of hydrofiber and polyurethane foam can be effectively used for treating a wide variety of burns. It presents a well-tolerated and easy to use alternative for the treatment of donor sites for skin grafting.

P148 Diagnostics and treatment with acute burn sepsis

BABUR Shakirov ${ }^{1}$, H Karabaev², K Tagaev², E Hakimov ${ }^{1}$

${ }^{1}$ Republican Scientific Centre of Urgent Medical Aid (RSCUMA), SAMARKAND, Uzbekistan

2Samarkand State Medical Institute, SAMARKAND, Uzbekistan

Objectives: Severe burn trauma causes the systematic inflammatory response syndrome, which leads to damage from possible sepsis and severe sepsis development. To determine the value of the procalcitonin test used for early diagnosis of sepsis and to study the course and treatment of burn sepsis in patients with severe burns.

Methods: Eighty patients in the Burns department of Republican Scientific Centre of Urgent Medical Aid (RSCUMA), aged 17-75 years with burn injuries covering $30 \%-85 \%$ of the body surface, were enrolled in the study. Procalcitonin is marker of sepsis, procalcitonin $>2 \mathrm{ng} / \mathrm{mL}$, sensitivity $-89 \%$, specific feature $-94 \%$.

Results: The result showed that among septic patients with severe burns, rational use of intensive therapy for burn sepsis and septic shock in combination with parentrial ozonotherapy resulted in decreases of syndrome of poly organ insufficiency and lethal outcomes from $70 \%$ accordingly. The result allows the conclusion that the treatment examined leads to a significant increase in survival coefficient.

Conclusion: This in turn confirms the efficacy of early necrectomy and auto dermoplasty of deep burn wounds in victims with sepsis.

P149 Do dressing materials influence the healing time after enzymatic debridement?

\section{B. Hartmann, F. Sander}

Unfallkrankenhaus Berlin, BERLIN, Germany

Objectives: In the trauma hospital Berlin during the time from 2014 to 2016, 56 patients with 57 evaluated wounds were treated with enzymatic debridement. 22 had Polylactic membranes after enzyme application and 20 Silicone dressings.

Methods: We included 42 Patients, 31 male, and11 females, receiving enzymatic debridement within the period described in a retrospective cohortstudy.

Results: The average age was 44,45 years; no difference men /women. There were 32 flame burns and 7scalds, contact, electricity and electrical arch each 1 . The average TBSA was 8,1 in the Polylactic and 23,57 in the Silicone group. $(p=0,0016)$, ABSI was $\varnothing 4,39$ without significant difference. There was no significant difference in the need for escharotomies. $(\mathrm{p} 0,26)$. Nexobrid was applied on average after 3,33 days, mostly under general anesthesia in an area of $\varnothing 1110 \mathrm{~cm}^{2}$ or $5,1 \%$, using in avg. $9.02 \mathrm{~g}$ of debridement powder. Post soaking periods was $\varnothing 4,2$ hours and $\mathrm{Hb}$ - difference from pre to 
postoperative 1,3g/l. Pseudo-eschar could be found in 6/16 in the Polylactide and 5/15 in the Silicone group (no sig.) Residual necrosis was higher in the Silicone group ( 0,111 versus $0,8 \%$ ). Residual necrosis was removed in $27 \%$ of the Polylactide group and $58 \%$ of the Silicone group before dressing.

Infection rate after $\varnothing 17$ days was $80 \%$ in the Silicone group and $37 \%$ in the Polylactide group. (Silver cream had an infection rate of $100 \%$ in 5Pat.) There was no significant difference between groups in time to transplant (Ø16,68 days) of mostly split skin. The average time to $95 \%$ healing was in the polylactic group 22 days and the silicone group 46 days. As in the silicone, the group showed a significantly higher TBSA we compared the patients with a TBSA of $<15 \%$ (Mean of TBSA). In this part, the time to $95 \%$ healing was 21 in the Polylactic versus 38 days in the Silicone group.

Conclusion: Polylactic membranes proofed to be at least adequate dressing compared to Silicone both in aspects of healing time and infection rate.

P150 An innovative dressing for a burn caused when a cellphone exploded with use of a Epicyte hydro dressing

M.C. Serra do Valle freitas ${ }^{1}, \underline{B}^{\text {B Bolgani }}{ }^{2}$, A.P. Ardila Bernal ${ }^{3}$, T. Arrabella Borgues ${ }^{4}$

${ }^{1}$ Souza Aguiar Hospital, Brazil

${ }^{2}$ Alemán Hospital, BUENOS AIRES, Argentina

3Ivo Pitanguy Intitute, RIO DE JANEIRO, Brazil

${ }^{4}$ Ivo Pitanguy Institute, RIO DE JANEIRO, Brazil

Objectives: To analyse the pain and quantity of time of scarring with use of an Epicyte hydro dressing

Methods: Clinical case: An 11 year old boy who got burned when his cellphone exploded in his pocket while he was playing football. He had an intermediate 2nd degree burn yet he suffered a lot of pain when compared to other patients with this degree of burn. The patient had been received in the Souza Aguiar burns unit 4 days post burn in a lot of pain. His parents did not consent for a skin graft to be used. The dressing he had was carefully removed and Epicyte hydro special dressing was applied where the patient instantly felt relief.

The period that the patient remained with the dressings was 7-14 days. He did not need any analgesics and the dressing was needed to be changed three times during this period, as with movement, the dressing would slide leaving the area uncovered.

Results: With difficulties such as calming the parent's anxieties and with fear of pain from the boy, with just 3 dressing changes the scarring had a favorable result.

Conclusion: Despite this being a difficult case, the Epicyte hydro dressing showed efficiency to facilitate the dressing change, relieve pain and demonstrate scarring of good quality.

Picture 1: https://www.eventure-online.com/parthen-uploads/89/9EBA/add_546137_bcfdb3713ae1-4f2a-a189-30a43ce5e051.jpg

Picture 2: https://www.eventure-online.com/parthen-uploads/89/9EBA/add_1_546137_bcfdb3713ae1-4f2a-a189-30a43ce5e051.jpg

Picture 3: https://www.eventure-online.com/parthen-uploads/89/9EBA/add_2_546137_bcfdb3713ae1-4f2a-a189-30a43ce5e051.jpg

\section{P151 BURNED PATIENT'S PAIN, A STRATEGY TO RELIEVE.}

A.P Ardila Bernal ${ }^{1}$, M.C. Do Valle Serra ${ }^{2}$, I. Daher Barra ${ }^{2}$, K. Vazan Maio R. ${ }^{2}$, T. Arrabella Borgues ${ }^{3}$ ${ }^{1}$ Ivo Pitanguy Intitute, RIO DE JANEIRO., Brazil

${ }^{2}$ Souza Aguiar Municipal Hospital, RIO DE JANEIRO, Brazil

${ }^{3}$ Ivo Pitanguy Institute, RIO DE JANEIRO, Brazil

Objectives: To evaluate the pain and recovery of burned patients with the use of special dressings that can remain from 7 to 14 days on 2 nd degree burns.

Methods: A prospective observational study where variables such as pain, hospitalization time and cost were analyzed using cellulose based dressings containing $95 \%$ of water in their composition, in burned patients. The sample was of 14 patients, the age ranged from 2 to 51 years ( $X=26$ years). Patients of both sexes with 8 women (57\%). The causal agents of the burn were: oil (5), fire (6), electricity 
(1), others (2). All patients had superficial and intermediate second degree burns. SCQ ranged from 5\% to $80 \%(X=35 \%)$. The period that the patients remained with dressings was 7-14 days with daily revisions. Pain was assessed by visual analogue scale (VAS). Exclusion criteria were 1 degree and 3 degree burns.

Results: We found a greater comfort and well-being of patients immediately after dressing placement, improved pain tolerance and a notable decrease in the need for analgesic use. There was even less need to change daily dressings avoiding direct trauma to the wound. We also observed early healing of burned areas, allowing early dehospitalization, generating the possibility of progressing patients to the ambulatory regimen, with weekly return.

Conclusion: The dressing showed to be effective in managing the pain of the burned patient with less traumatic recovery, earlier epithelization and fewer psychological sequelae.

Picture 1: https://www.eventure-online.com/parthen-uploads/89/9EBA/add_1_546149_6f17ac804cdf-42c5-8364-12b63d9e28ca.png

Picture 2: https://www.eventure-online.com/parthen-uploads/89/9EBA/add_546149_6f17ac804cdf-42c5-8364-12b63d9e28ca.jpg

P152 Management of full-thickness skin defects in the upper extremity: first short-term experiences with the acellular dermal substitute

MA Argirova ${ }^{1}$, YZ Zayakova $^{2}$, TD Dimitrova ${ }^{1}$, AV Viktorova ${ }^{3}$

${ }^{1}$ UMHATEM „N.I. Pirogov,", SOFIA, Bulgaria

${ }^{2}$ Department of burns and plastic surgery, VARNA, Bulgaria

${ }^{3}$ Clinic of burns and plastic surgery, SOFIA, Bulgaria

Objectives: The aimof the study is to present our short-term experiences with collagenand elastin dermal matrix - Matriderm ${ }^{\circledR}$ in the management of full-thickness skin defects in the upper extremity.

Methods: Between August 2018 and February 2019, a retrospective review of patients with fullthickness skin defects in the functional region of the upper extremity was performed. All the patients were treated with the dermal substitute Matriderm ${ }^{\circledR}$ with $1 \mathrm{~mm}$ thickness, and unmeshed skin graft in a single-step procedure. We examined the efficacy and graft survival rate and evaluated postoperative functional skin values. Individual comparisons were performed between the area of skin graft and the surrounding normal skin. Early evaluation of the wounds took place on day 5 and 9, determining the take rate of the skin grafts in percentage. Their case notes were reviewed and data was collected - age, injury mechanism, site, and defect size.

Results: A total of 9 patients were enrolled in this study. The mean age was $34.55 \pm 17.51$ years. Causes of the defects included correction of scar contracture $(n=4)$, degloving injury $(n=3)$ and highenergy trauma $(\mathrm{n}=2)$. The size of soft tissue defects was between 120 and $300 \mathrm{~cm}^{2}(235 \pm 79.37)$. There were no complications or inflammatory response in all cases but one, which resulted in a seroma. All wounds were epithelized within 11 days without additional grafting. The overall survival rate of the matrix and the skin graft was $97.55 \pm 2.60 \%$. The average VSS score was 1.97 .

Conclusion: Managing the upper extremity injuries is complex and requires careful planning to provide stable coverage by the safest and least invasive method. Matriderm ${ }^{\circledR}$ represents a valuable alternative to other types of defect coverage and should be considered in the treatment of full-thickness skin defects.

P153 Letters from the lactide frontiers 2019

\section{$\underline{\text { H. Haller }}$}

HLmedConsult, LEONDING, Austria

Objectives: Polymers from mixtures of lactic acid are used in burns treatment for nearly twenty years. They have established as a well-recognized treatment method with short or even shorter healing time, low complications and excellent cosmetic results in superficial, deep partial thickness and even small full thickness burns. The fact of pain reduction accompanies this. Lots of studies proved this; interestingly nobody answered the question about the reason for this.

Methods: This presentation will try to explain the "Lactate Effect" in burns treatment and correlate 
this to clinical effects. We know that polylactides degenerate to chemical fragments releasing lactate and lactic acid.

Lactate and lactic acid serve as an energy source for the cells. Both substances can permeate the cell membranes either due to their molecule size or to active transport by the monocarboxylate transporter (MCT) protein shuttle system. Inside of the cell, lactate can serve as an energy source via the Cori cycle or lactate can oxidize to pyruvate (via lactate dehydrogenase) which is then can oxidize to acetyl-CoA and fuels the TCA cycle in mitochondria producing the metabolic products carbon dioxide, water, and NADH which provides the energy-rich NAD.

Lactate and pyruvate act as potent antioxidants within the cells. Medical literature describes the role of oxidative stress in an increasing manner.

Lactate acts on cytokines and increases TGF $\beta$ activation, Hypoxia Inducible Factor, VEGF, and others as many studies showed.

Results: Polylactic membranes act as activators in wound healing and support curing by supplementation of energy. Telomeres conservation will contribute to a reduction of cell-aging and eternization, maintaining the rate of duplications and the quality of the skin. TGF $\beta$ affects keratinocytes, completing the activation status for healing. In dermal tissue, it increases fibroblast growth, collagen, and extracellular matrix synthesis as well as neovascularization. It acts as a radical scavenger as well. Clinical findings bolster these laboratory results.

Conclusion: Using these effects enables you to use polylactides as a toolbox for a broad range of indications!

\section{IF5.05 Hydrotherapy in burn centres. Pro \& cons.}

Jacques Latarjet, St. Luc Lyon Burn Centre, France

Although hydrotherapy for the local treatment of the burn wound at the acute stage was already mentioned in the thirties, the method consisting in immersing the burned patient in a Hubbard tank filled with warm saline water was first used extensively by Mac Indoe and Gillies to treat RAF pilots in East Grinstead in 1940, mainly for comfort and pain control during dressing changes.Since then,hydrotherapy seems to have been the gold standard in local burn care, all new burn centres being equipped with more and more sophisticated Hubbard tanks or showering trolleys in specifically designed hydrotherapy rooms.

With the high risk of deadly sepsis in extensive deep burns, due more and more often to multidrug resistant organisms, there has been periodically serious concerns about the validity of these practices which seem to rely more on tradition than on scientific evidence.

In 1987, in Lyon St Luc Burn centre, we decided with Dr. Foyatier to stop definitively all kind of hydrotherapy, obtaining thus a significant decrease of Pseudomonas infections and septicaemias, reported at the New Delhi ISBI conference in 1990. In 1992, in a quasi historical study, (C.I.D 1992;15:941-9, 1992), E. Tredget, in Edmonton (Alberta), achieved, by giving up hydrotherapy, an impressive diminution of the mortality due to sepsis, with a no death score for pseudomonas. Despite these results and the regular reports of infection outbreaks incriminating the use of water, a study (Davison P.\&al., J Burn Care Res 2010;31:393-399) conducted in 2010 in 44\% of 142 North American burn centres showed that $83 \%$ were still using hydrotherapy (10/59 centres did not). In 2014, in UK and Ireland (Langschmidt J. et. al, Burns, 40, (5)860-4) this was also the case for 27 out of 28 burn care providers (Immersion in tank for 21).

A recent metanalysis (CID 2017:64,1435-1443, K. Gordon et al) shows that hospital water supply systems are an important source of nosocomial infections by multidrug resistant organisms, especially by Pseudomonas, for all kind of pathologies, with a high mortality rate, and the impossibility to eradicate the responsible germ in $1 / 3$ study. Because bacteria can survive for a very long time in water, can produce contaminating biofilms and even exchange plasmids in environmental reservoirs, the bacteriological control of hospital water delivery, despite unceasing and costly controls and protocolized care, can only be approximate.

Conclusions: (1) Sepsis is nowadays the leading cause of death in burns 
(2) Hydrotherapy, whose benefits over other methods of local treatment rely exclusively on tradition increases the risk of sepsis in burns.

(C) 2019 by the authors. Submitted for possible open access publication under the terms and conditions of the Creative Commons Attribution (CC BY) license (http://creativecommons.org/licenses/by/4.0/). 